NTP Monograph

on the Systematic Review of Occupational

Exposure to Cancer

Chemotherapy

Agents and Adverse Health Outcomes

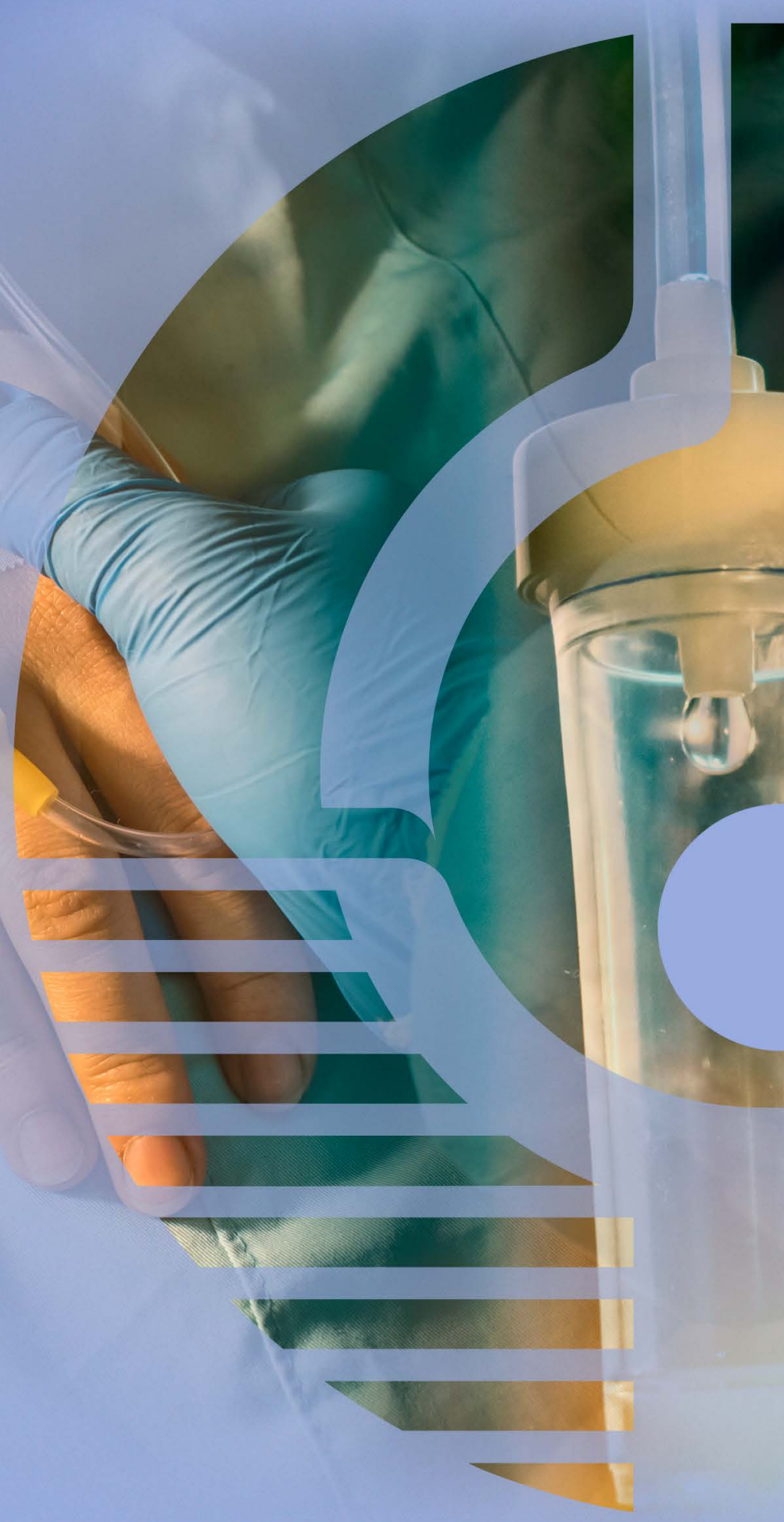




\title{
NTP Monograph on the Systematic Review of Occupational Exposure to Cancer Chemotherapy Agents and Adverse Health Outcomes
}

NTP Monograph 05

March 2019

\author{
National Toxicology Program \\ Public Health Service \\ U.S. Department of Health and Human Services
}

ISSN: 2378-5144

Research Triangle Park, North Carolina, USA 


\section{Foreword}

The National Toxicology Program (NTP), established in 1978, is an interagency program within the Public Health Service of the U.S. Department of Health and Human Services. Its activities are executed through a partnership of the National Institute for Occupational Safety and Health (part of the Centers for Disease Control and Prevention), the Food and Drug Administration (primarily at the National Center for Toxicological Research), and the National Institute of Environmental Health Sciences (part of the National Institutes of Health), where the program is administratively located. NTP offers a unique venue for the testing, research, and analysis of agents of concern to identify toxic and biological effects, provide information that strengthens the science base, and inform decisions by health regulatory and research agencies to safeguard public health. NTP also works to develop and apply new and improved methods and approaches that advance toxicology and better assess health effects from environmental exposures.

NTP conducts literature-based evaluations to determine whether exposure to environmental substances (e.g., chemicals, physical agents, and mixtures) may be associated with adverse health effects. These evaluations result in hazard conclusions or characterize the extent of the evidence and are published in the NTP Monograph series, which began in 2011. NTP Monographs serve as an environmental health resource to provide information that can used to make informed decisions about whether exposure to a substance may be of concern for human health.

NTP conducts these health effects evaluations following pre-specified protocols that apply the general methods outlined in the "Handbook for Conducting a Literature-Based Health Assessment Using the OHAT Approach for Systematic Review and Evidence Integration." † The protocol describes project-specific procedures tailored to each systematic review in a process that facilitates evaluation and integration of scientific evidence from published human, experimental animal, and mechanistic studies.

The key feature of the systematic review approach is the application of a transparent framework to document the evaluation methods and the basis for scientific judgements. This process includes steps to comprehensively search for studies, select relevant evidence, assess individual study quality, rate confidence in bodies of evidence across studies, and then integrate evidence to develop conclusions for the specific research question. Draft monographs undergo external peer review prior to being finalized and published.

NTP Monographs are available free of charge on the NTP website and cataloged in PubMed, a free resource developed and maintained by the National Library of Medicine (part of the National Institutes of Health). Data for these evaluations are included in the Health Assessment and Workspace Collaborative.

For questions about the monographs, please email NTP or call 984-287-3211.

${ }^{\dagger}$ OHAT is the abbreviation for Office of Health Assessment and Translation, which is within the Division of the National Toxicology Program at the National Institute of Environmental Health Sciences. 


\section{Table of Contents}

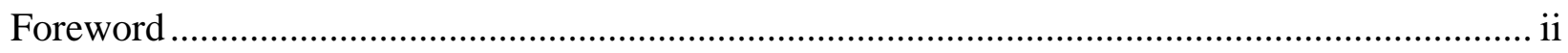

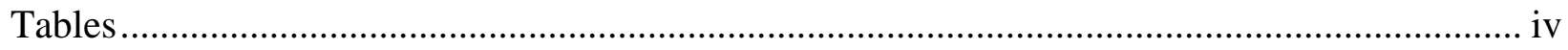

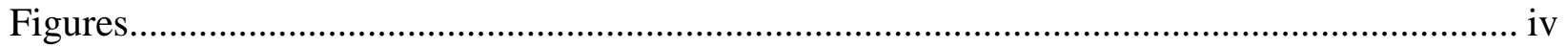

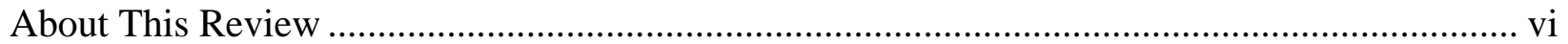

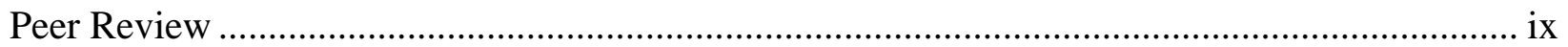

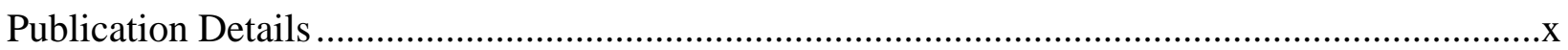

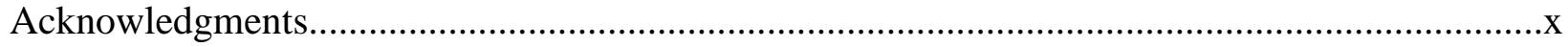

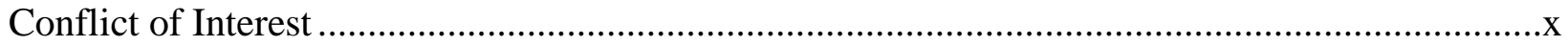

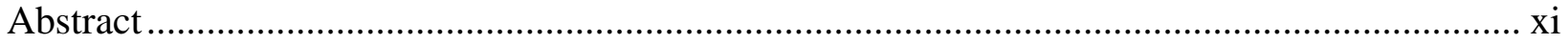

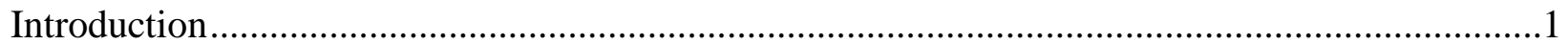

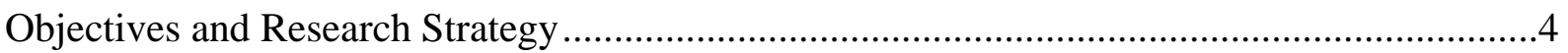

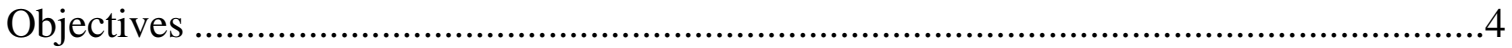

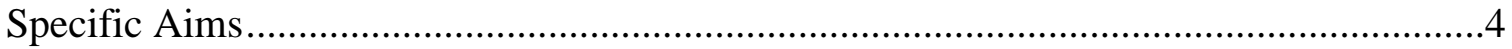

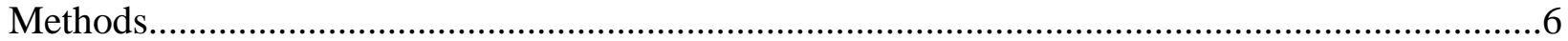

Problem Formulation and Protocol Development ………...................................................6

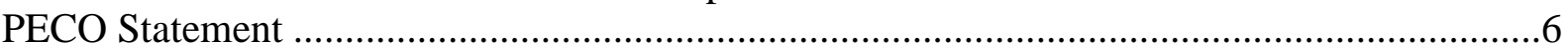

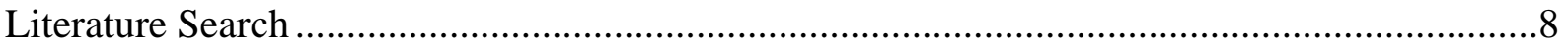

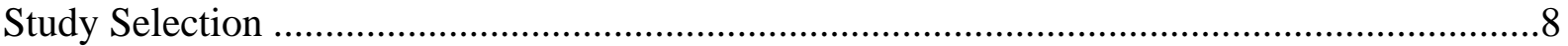

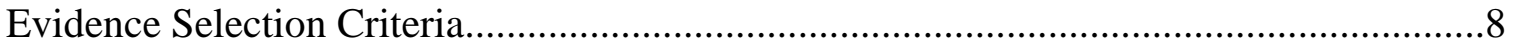

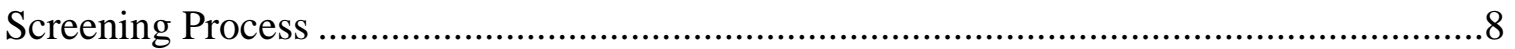

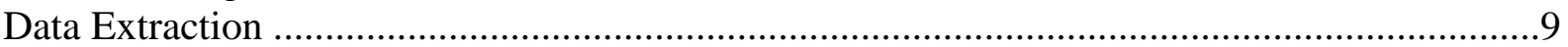

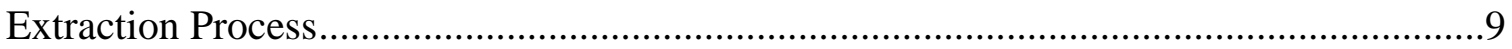

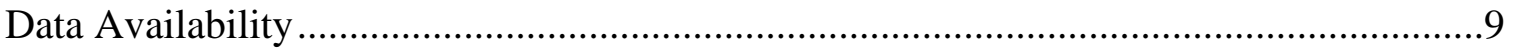

Quality Assessment of Individual Studies ..........................................................................

Rating Confidence in Bodies of Evidence ..............................................................................10

Adjustment of Initial Confidence Ratings of Cross-sectional Studies Evaluating

Genetic Toxicity.....................................................................................................

Preparation of Level-of-evidence Conclusions ......................................................................12

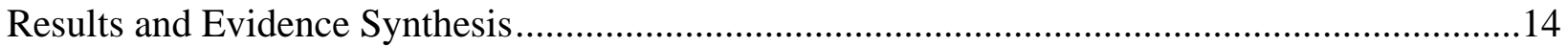

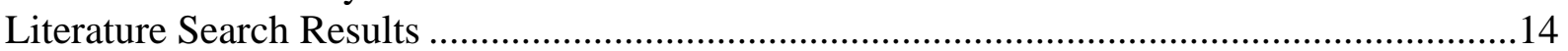

Health Outcomes with Inadequate Evidence ..................................................................16

Spontaneous Abortion.............................................................................................16

Genetic Toxicity: DNA Damage .................................................................................

Evidence of Occupational Exposure to Cancer Chemotherapy Agents................................................4

Environmental Monitoring..........................................................................................4

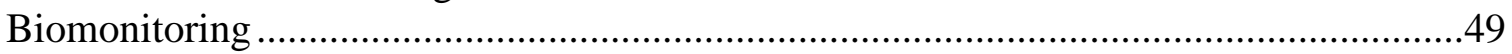

Other Occupationally Exposed Populations ……………………………………………...51

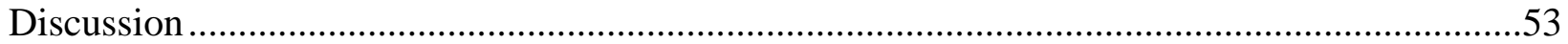

Impact of Exposure Protection Efforts............................................................................... 


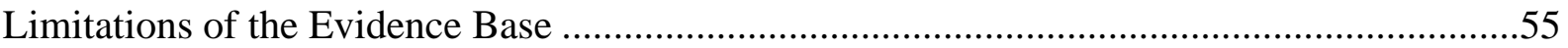

Study Design Limitations ............................................................................................5

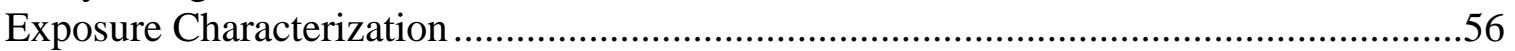

Challenges in Evaluating Reproductive Toxicity ...........................................................57

Limitations of the Systematic Review ………………...........................................................57

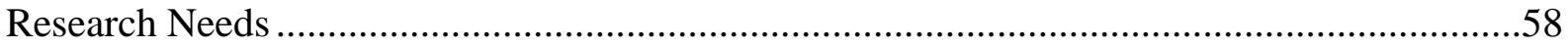

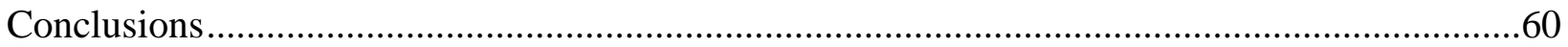

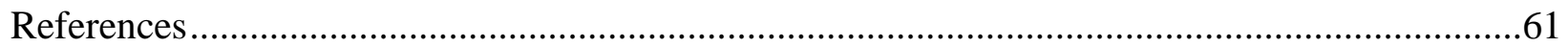

Appendix A. Literature Search Strategy …………............................................................. A-1

Appendix B. List of Included Studies Reporting Health Outcomes ...............................................

Appendix C. Risk-of-bias Assessment for All Included Studies ......................................................

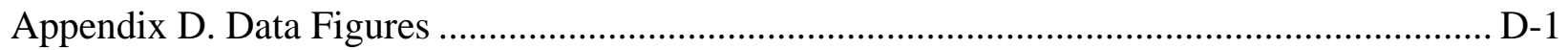

Appendix E. Inadequate Evidence: Evidence Synthesis and Risk-of-bias Assessment ................E-1

Appendix F. Studies Reporting on Genetic Toxicity ............................................................

Appendix G. List of Included Studies Reporting on Environmental Monitoring or

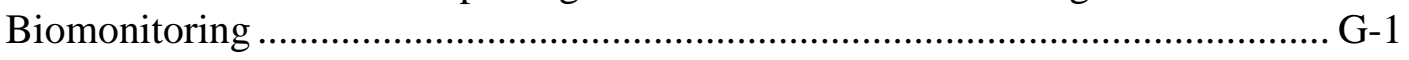

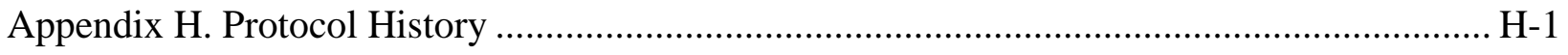

Appendix I. Supplemental Files...........................................................................................

\section{Tables}

Table 1. Carcinogenicity, Mutagenicity, and Developmental Toxicity of Cancer Chemotherapy Agents by Drug Class Based on Direct, Intentional Exposure to Humans and Laboratory Animals .....................................................................................

Table 2. PECO Statement for Objective 1 ………...............................................................6

Table 3. Definitions of Level-of-evidence Descriptors ..................................................................13

Table 4. Categories of Health Outcomes for 110 Included Studies...................................................16

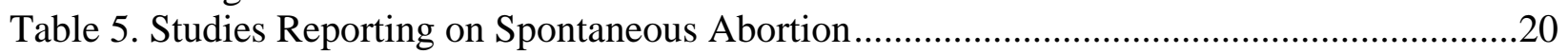

\section{Figures}

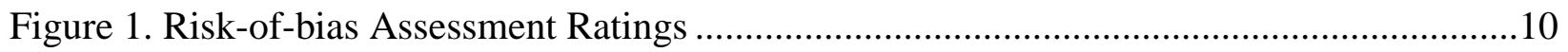

Figure 2. Assessing Confidence in the Body of Evidence...............................................................12

Figure 3. Translation of Confidence Ratings into Evidence of Health Effect Conclusions ...........12

Figure 4. Study Selection Diagram .........................................................................................14

Figure 5. Confidence in the Body of Evidence for Spontaneous Abortions....................................31

Figure 6. Confidence in the Body of Evidence for Structural Chromosomal Aberrations

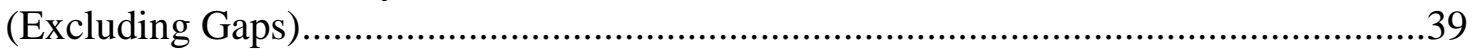

Figure 7. Confidence in the Body of Evidence for Micronucleus Induction...................................43

Figure 8. Confidence in the Body of Evidence for DNA Damage Measured by the Comet

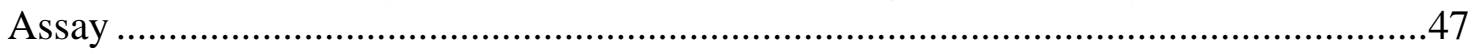


Figure 9. Maximum Levels of Cyclophosphamide Contamination Reported for the Biological Safety Cabinet Workspace and Floor in Front of the Biological Safety Cabinet from 1993 to 2016 ....

Figure 10. Percent of Workers with Cyclophosphamide Detected Above the Level of Detection in Urine Samples Displayed by the Year Samples Were Collected (1985-2016)

This report has been reformatted to meet new NTP publishing requirements; its content has not changed. 


\section{About This Review}

National Toxicology Program ${ }^{1}$

${ }^{1}$ Division of the National Toxicology Program, National Institute of Environmental Health

Sciences, Research Triangle Park, North Carolina, USA

\section{Collaborators}

Kembra L. Howdeshell, Michael D. Shelby, Robyn B. Blain, Ruth M. Lunn, Pam Ross, Kyla W. Taylor, Kristina A. Thayer, Kristine L. Witt, Andrew A. Rooney

Division of the National Toxicology Program, National Institute of Environmental Health Sciences, Research Triangle Park, North Carolina, USA

Contributed to conception or design and contributed to drafting of protocol

Kembra L. Howdeshell, Ph.D., Project Lead

Andrew A. Rooney, Ph.D.

Michael D. Shelby, Ph.D.

Contributed to drafting of report

Kembra L. Howdeshell, Ph.D.

Andrew A. Rooney, Ph.D.

Michael D. Shelby, Ph.D.

Kyla W. Taylor, Ph.D.

Critically reviewed draft report and figures

Ruth M. Lunn, Ph.D.

Critically reviewed protocol, draft report, and figures

Kristine L. Witt, M.S.

Retrieved and managed references

Kembra L. Howdeshell, Ph.D.

Screened studies

Kembra L. Howdeshell, Ph.D.

Michael D. Shelby, Ph.D.

Developed visualizations

Kembra L. Howdeshell, Ph.D.

Assessed risk of bias

Kyla W. Taylor, Ph.D.

ICF, Durham, North Carolina, USA

Critically reviewed draft report and figures

Robyn B. Blain, Ph.D. 
Pam Ross, M.S., Lead Work Assignment Manager

Extracted data and assessed risk of bias

Robyn B. Blain, Ph.D.

Pam Ross, M.S.

National Center for Environmental Assessment, U.S. Environmental Protection Agency

(formerly of Division of the National Toxicology Program, National Institute of

Environmental Health Sciences), Research Triangle Park, North Carolina, USA

Contributed to conception or design, contributed to drafting of protocol, and contributed to drafting of report

Kristina A. Thayer, Ph.D.

\section{Contributors}

Division of the National Toxicology Program, National Institute of Environmental Health

Sciences, Research Triangle Park, North Carolina, USA

Critically reviewed protocol

Grace E. Kissling, Ph.D.

Barry S. McIntyre, Ph.D.

Critically reviewed draft report and figures

Brandiese E. J. Beverly, Ph.D.

Windy A. Boyd, Ph.D.

Vickie R. Walker, B.S.

Coordinated peer review

Mary S. Wolfe, Ph.D.

ICF, Durham, North Carolina, USA

Contract oversight

David F. Burch, M.E.M.

Coordinated peer review

Canden N. Byrd, B.S.

Edited and formatted report

Tara Hamilton, M.S.

Katherine R. Helmick, M.P.H.

Whitney K. Mitchell, B.S.

Extracted data

Ali Goldstone, M.P.H.

Sofie McComb, B.S.

Devon Morgan, B.S.

Chris Sibrizzi, M.S. 
Assessed risk of bias

Sorina E. Eftim, Ph.D.

Ali Goldstone, M.P.H.

Chris Sibrizzi, M.S.

Division of Surveillance, Hazard Evaluations, and Field Studies, National Institute for Occupational Safety and Health, Cincinnati, Ohio, USA

Critically reviewed protocol and critically reviewed draft report and figures

Thomas H. Connor, Ph.D.

Christina C. Lawson, Ph.D.

Office of Scientific Information Management, National Institute of Environmental Health

Sciences, Durham, North Carolina, USA

Designed and executed literature searches

Stephanie D. Holmgren, M.S.L.S., MBA

Office of the Assistant Secretary for Preparedness and Response, U.S. Department of Health and Human Services (formerly of Occupational Safety and Health Administration), Washington, District of Columbia, USA

Critically reviewed protocol and critically reviewed draft report and figures

Margy Lambert, Ph.D.

U.S. Food and Drug Administration, Silver Spring, Maryland, USA

Critically reviewed protocol and critically reviewed draft report and figures

John K. Leighton, Ph.D.

National Cancer Institute, Rockville, Maryland, USA

Critically reviewed protocol and critically reviewed draft report and figures

Harold Seifried, Ph.D.

Nonnekaye Shelburne, M.S., CRNP

Social \& Scientific Systems, Incorporated, Durham, North Carolina, USA

Extracted data

Fikri Yucel, M.S.

University of Maryland School of Medicine, Baltimore, Maryland, USA

Critically reviewed protocol and critically reviewed draft report and figures

Melissa A. McDiarmid, M.D.

Logistics Health Incorporated, La Crosse, Wisconsin, USA

Critically reviewed protocol

William G. Buchta, M.D. (formerly of Division of Preventive, Occupational, and Aerospace

Medicine, Mayo Clinic)

University of California-San Francisco, San Francisco, California, USA

Critically reviewed protocol

Patrice Sutton, M.P.H. 


\section{Peer Review}

The National Toxicology Program (NTP) conducted a peer review of the draft NTP Systematic Review of Occupational Exposure to Cancer Chemotherapy Agents and Adverse Health Outcomes by letter in March 2018 by the experts listed below. Reviewer selection and document review followed established NTP practices. The reviewers were charged to:

(1) Determine whether the scientific information cited in the draft monograph is technically correct and clearly stated, and whether NTP has objectively presented and assessed the scientific evidence.

(2) Determine whether the scientific evidence presented in the draft monograph supports NTP's conclusions regarding the level of evidence from human studies for adverse health effects associated with occupational exposure to cancer chemotherapy agents.

(3) Review and comment on NTP's presentation of workplace monitoring and biomonitoring data as well as proposed research needs based on the assessment of the available scientific data on health effects and exposure monitoring.

NTP carefully considered reviewer comments in finalizing this monograph.

\section{Peer Reviewers}

\section{David A. Eastmond, Ph.D.}

Environmental Toxicology Graduate Program

Department of Molecular, Cell and Systems Biology

University of California-Riverside

Riverside, California, USA

\section{S. Katharine Hammond, Ph.D., CIH}

School of Public Health, Environmental Health Sciences Division

University of California-Berkeley

Berkeley, California, USA

\section{Steven Markowitz, M.D., Dr.P.H.}

Barry Commoner Center for Health and the Environment

Queens College

Flushing, New York, USA

\section{Carol Rice, Ph.D.}

Department of Environmental Health

University of Cincinnati College of Medicine

Cincinnati, Ohio, USA

\section{Mark B. Russi, M.D., FACOEM}

Wellness and Employee Population Health

Yale School of Medicine and Yale-New Haven Health System

New Haven, Connecticut, USA 


\section{Publication Details}

Publisher: National Toxicology Program

Publishing Location: Research Triangle Park, NC

ISSN: 2378-5144

DOI: https://doi.org/10.22427/NTP-MGRAPH-5

Report Series: NTP Monograph Series

Report Series Number: 05

Official citation: National Toxicology Program (NTP). 2019. NTP monograph on the systematic review of occupational exposure to cancer chemotherapy agents and adverse health outcomes. Research Triangle Park, NC: National Toxicology Program. NTP Monograph 05.

\section{Acknowledgments}

This work was supported by the Intramural Research Program (ES103316, ES103317) at the National Institute of Environmental Health Sciences, National Institutes of Health and performed for the National Toxicology Program, Public Health Service, U.S. Department of Health and Human Services under contracts HHSN316201200028W (Order No. HHSN27300006 and Order No. HHSN27300002) and GS00Q14OADU417 (Order No. HHSN273201600015U).

\section{Conflict of Interest}

Individuals identified as collaborators in the About This Review section have certified that they have no known real or apparent conflict of interest related to cancer chemotherapy agents. 


\section{Abstract}

Introduction: Many cancer chemotherapy agents are known carcinogens, genetic toxicants, and developmental toxicants. Secondary malignancies, such as therapy-related acute myeloid leukemia, are caused by cancer chemotherapy agents administered to patients for the treatment of cancer. Occupational exposure to these agents was first documented in the 1970s and continues to occur, despite the issuance of safe handling guidelines in 1980s.

Objectives: Based on the evidence of carcinogenicity and genetic toxicity associated with direct administration of cancer chemotherapy agents and current evidence of occupational exposure, the National Toxicology Program (NTP) conducted a systematic review to: (1) evaluate whether occupational exposure (e.g., medical, manufacturing, research, and veterinary) is associated with any adverse health outcomes in humans, and (2) summarize the prevalence and levels of chemotherapy agents in the workplace as measured by environmental monitoring and biomonitoring for possible worker exposures.

Methods: The evaluation was conducted following the Office of Health Assessment and Translation (OHAT) method. A literature search was performed up to February 23, 2017, using PubMed, Embase, Scopus, Toxline, and Web of Science. Relevant human studies were data extracted and assessed for risk of bias. Bodies of evidence were assessed to develop confidence ratings and level-of-evidence conclusions that reflect the certainty in the evidence that occupational exposure to cancer chemotherapy agents are associated with health effects on a per outcome basis.

Results and Evidence Synthesis: The literature search and screening process identified 110 epidemiological studies relevant to assessing possible adverse health outcomes. Most studies addressing health outcomes evaluated potential DNA damage ( $n=66$; specifically, structural chromosomal aberrations (CA) and micronucleus (MN) induction and comet assay endpoints) and spontaneous abortion $(n=16)$. In addition to DNA damage, groups of studies were identified to evaluate the potential association between occupational exposure to cancer chemotherapy agents and adverse health outcomes, including cancer (three studies) and adverse effects on reproduction (30 studies). Additional health outcomes included acute effects, immune effects, and liver and kidney toxicity. One hundred sixty-one studies were identified to assess workplace exposure based on reporting of environmental contamination (107 studies) and urine and/or blood monitoring of these agents (82 studies).

Discussion and Conclusions: NTP concluded that there is a moderate level of evidence that occupational exposure to chemotherapy agents is associated with increased incidence of spontaneous abortion, particularly when evaluating studies of nursing and pharmacy personnel. NTP also concluded that there is a moderate level of evidence that exposure to chemotherapy agents in the workplace is associated with genetic toxicity in humans based on consistent reports significantly higher levels of structural CA (\% of cells with CA and number of CA), MN induction (number of cells with $\mathrm{MN}$ and number of $\mathrm{MN}$ ) and DNA damage measured by comet assay (\% tail DNA, tail length, tail moment, and DNA damage index) in exposed personnel. There was inadequate evidence for NTP to reach level-of-evidence conclusions on the remaining health outcomes, including cancer, primarily due to few studies per outcome and heterogeneity in the data. Despite current safety guidelines, cancer chemotherapy agents were commonly detected in environmental samples of the workplace (e.g., surface wipes and air sampling) and biosamples (e.g., urine or blood) of workers handling these agents, including data collected as 
recently as 2014 to 2016. Considering the potential for occupational exposure to these agents and the association between exposure and DNA damage and spontaneous abortions, there is a continued need to reduce exposures through training in safe handling procedures and provision and use of personal protective equipment and associated safety containment equipment. Health surveillance of occupationally exposed personnel would also benefit from improved exposure characterization methods, such as use of daily diaries that are assessed and validated to estimate exposure levels and additional environmental monitoring and biomonitoring data that include analytical chemistry approaches to assess multiple agents. There is also a need to better understand the sources (i.e., activities or physical locations) of worker exposure, especially in settings that have not been adequately studied (e.g., home care, veterinary clinics). 


\section{Introduction}

Cancer chemotherapy agents are designed to kill or halt the progression of cancer cells through various mechanisms of action. Due to their mechanisms of action, many cancer chemotherapy agents are reported to induce carcinogenicity and mutagenicity. Forty-two cancer chemotherapy agents are identified by the International Agency for Research on Cancer (IARC) as known $(\mathrm{n}=18$; IARC Class 1$)$, probable $(\mathrm{n}=11$; IARC Class $2 A)$, or possible $(\mathrm{n}=13$; IARC Class $2 \mathrm{~B})$ carcinogens in humans (IARC 2012; 2016). Cancer chemotherapy agents administered therapeutically for the treatment of cancer are known to cause secondary cancers, primarily therapy-related acute myeloid leukemia (Mistry et al. 2005; Morton et al. 2013; PedersenBjergaard 2005; Pedersen-Bjergaard et al. 2002; Sill et al. 2011). In addition, most cancer chemotherapy agents are reported to be teratogenic and/or to induce embryo toxicity in experimental studies of laboratory animal models and limited observational reports in women administered cancer chemotherapy for treatment of cancer during pregnancy [reviewed in (NTP 2013)]. A summary of the published carcinogenicity, mutagenicity, teratogenicity, and embryo toxicity of different cancer chemotherapy drug classes following direct, intentional exposure of these agents to human patients or experimental animals are presented in Table 1. Given the observed adverse health outcomes observed following intentional, direct administration of these agents to patients, the National Toxicology Program (NTP) evaluated the literature for potential adverse health outcomes in employees who are occupationally exposed to cancer chemotherapy agents.

Table 1. Carcinogenicity, Mutagenicity, and Developmental Toxicity of Cancer Chemotherapy Agents by Drug Class Based on Direct, Intentional Exposure to Humans and Laboratory Animals

\begin{tabular}{|c|c|c|c|c|}
\hline \multirow{2}{*}{ Drug Class $^{a}$} & \multirow{2}{*}{ Carcinogenicity $^{\mathbf{b}}$} & \multirow{2}{*}{$\begin{array}{c}\text { Genetic } \\
\text { Toxicity }\end{array}$} & \multicolumn{2}{|c|}{ Developmental Toxicity ${ }^{d}$} \\
\hline & & & Teratogenicity & Embryotoxicity \\
\hline Alkylating Agents & $+^{e}$ & + & + & + \\
\hline Antimetabolites & + & + & + & + \\
\hline Biological & inadequate data ${ }^{\mathrm{f}}$ & $-\mathrm{g}$ & - & $-\mathrm{h}$ \\
\hline Anthracycline Antibiotics & + & + & + & + \\
\hline Hormone Therapies & + & + & + & + \\
\hline Mitotic Function Inhibitors & inadequate data & + & + & + \\
\hline $\begin{array}{l}\text { Topoisomerase II Function } \\
\text { Inhibitors }\end{array}$ & + & + & + & + \\
\hline Tyrosine Kinase Inhibitors & inadequate data & inadequate data & + & + \\
\hline \multicolumn{5}{|c|}{$\begin{array}{l}\text { axamples of agents in each drug class: alkylating agents (e.g., cyclophosphamide), antimetabolites (e.g., hydroxyurea), } \\
\text { biologicals (e.g., cytokines, such as interferon alpha, and monoclonal antibodies, such as trastuzumab), anthracycline antibiotics } \\
\text { (e.g., doxorubicin), hormone therapies (e.g., tamoxifen), mitotic function inhibitors (e.g., vincristine), topoisomerase function } \\
\text { inhibitors (e.g., etoposide), and tyrosine kinase inhibitors (e.g., imatinib, adalimumab). } \\
\text { bData reviewed in: (IARC 1966; 1980; 1981; 1990; 2000). } \\
\text { 'Data reviewed in: (NTP 2008; 2016). } \\
\text { d'Data reviewed in: (NTP 2013). } \\
\text { eEvidence of an effect for at least one agent in this drug class. } \\
\text { fInadequate data; inconsistent data or a lack of data for one or more agents in this drug class. } \\
\text { gLack of an effect for agents in this drug class. } \\
\text { hTrastuzumab is a reproductive developmental toxicant, which induced oligohydramnios when administered in second and/or } \\
\text { third trimester. }\end{array}$} \\
\hline
\end{tabular}


Occupational exposure to cancer chemotherapy agents may occur in medical, veterinary, and manufacturing settings among personnel involved in the production, preparation and administration of these agents (Hall et al. 2013; Kiffmeyer et al. 2013; Kopp et al. 2013b) as well as other workers involved with the care of patients administered chemotherapy (e.g., unit clerks, volunteers, ward aides, dieticians, and shipper/receivers) (Hon et al. 2014). Potential routes of occupational exposure to these agents include dermal, ingestion, and inhalation. While levels of such exposures are thought to be much lower than those administered to cancer patients, occupational exposure likely involves more than one chemotherapy agent or combination therapy, and it may occur more frequently and over a longer time. Furthermore, occupational exposures are often unrecognized due to lack of systematic environmental monitoring and biomonitoring programs (OSHA 1999).

Evidence for occupational exposure began appearing in the 1970s with reports of elevated mutagenic activity in the urine of health care workers who prepared and administered such agents [reviewed in (Connor and McDiarmid 2006)]. Subsequent studies reported evidence of genetic toxicity such as chromosome aberrations, sister chromatid exchanges, and DNA damage in workers handling these agents, as well as the detection of cancer chemotherapy agents or their metabolites in workers' urine. Guidelines for safe handling of cancer chemotherapy agents were first published in the 1980s by national health care worker agencies in Australia and the United States [reviewed in (Connor and McDiarmid 2006)]. The Occupational Safety and Health Administration in the United States published guidelines for safe handling of cancer chemotherapy agents in 1986 (OSHA 1986), and the guidelines were updated in 1999 to include all hazardous drugs (OSHA 1999). Beginning in the 1990s, numerous publications documented surface contamination of safety cabinets, countertops, floors, and equipment with cancer chemotherapy agents. While improved handling procedures and engineering controls have reduced contamination, Connor et al. (2010) reported that surface contamination persists in pharmacy and nursing areas of some hospital-based cancer centers. In addition, the potential for occupational exposure to cancer chemotherapy agents has increased with greater usage of chemotherapy for non-cancer disease conditions [e.g., autoimmune diseases (Zack 2012)], the development of new surgical techniques involving administration of chemotherapy directly into the peritoneal cavity (Villa et al. 2015), and greater usage of chemotherapy by veterinary clinics to treat cancer in companion animals (Klahn 2014).

A number of health outcomes have been assessed in health care workers exposed to cancer chemotherapy agents, including cancer, genetic damage, reproductive and pregnancy outcomes, and acute effects (e.g., hair loss, nausea) [reviewed in (Connor and McDiarmid 2006)]. The association between occupational exposure to cancer chemotherapy agents and some of these health effects have been evaluated in a literature review and meta-analysis (Dranitsaris et al. 2005), a systematic review (Quansah and Jaakkola 2010), and a recent narrative literature review by Connor et al. (2014). The review and meta-analysis by Dranitsaris and colleagues (2005) evaluated the literature on cancer, pregnancy outcomes, and acute toxic effects in nurses, pharmacists, and pharmacy technicians/assistants who work with cancer chemotherapy agents. They reported that there was insufficient literature to reach conclusions on cancer, acute effects, congenital malformations, or stillbirth; however, they did find a significant association between occupational exposure and spontaneous abortions (odds ratio $(\mathrm{OR})=1.46,95 \%$ confidence interval $(\mathrm{CI})=1.11-1.92$ ) (Dranitsaris et al. 2005). A systematic review and meta-analysis by Quansah and Jaakkola (2010) evaluated the association between occupational exposures and 
work conditions of nurses only, including cancer chemotherapy agents and risk of spontaneous abortion. The meta-analysis on spontaneous abortion by Quansah and Jaakkola (2010) did not reach statistical significance $(\mathrm{OR}=1.21,95 \% \mathrm{CI}=0.80-1.83)$; however, they included a different combination of studies than the systematic review by Dranitsaris et al. (2005). Finally, a narrative review by Connor et al. (2014) provided a survey of the literature on occupational exposure to cancer chemotherapy agents and reproductive health, including spontaneous abortion, congenital anomalies as well as other reproductive outcomes (e.g., fertility, time to pregnancy, birth weight). Overall, confidence in the findings was limited for outcomes other than spontaneous abortion (e.g., congenital malformations) due to low sample size and heterogeneity in the study designs (Connor et al. 2014; Dranitsaris et al. 2005; Quansah and Jaakkola 2010).

Accurate characterization of exposure is one of the major challenges in assessing the adverse health effects of occupational exposure to cancer chemotherapy. Occupational exposure to cancer chemotherapy agents is a complex exposure involving many different cancer chemotherapy agents per day or per week, and each agent may have different potencies to induce the same or different adverse health effects. Furthermore, usage of personal protective equipment and safety containment equipment can influence the level of occupational exposure. Exposure is generally characterized by job title or industrial hygiene data in occupational settings; however, this information alone (i.e., job title or position) does not provide much detail on level of exposure. More informative approaches for exposure characterization of occupational exposure to cancer chemotherapy agents include the use of a validated questionnaire or use of a daily diary where the subject reports on the types of drugs handled, levels handled (i.e., doses administered), frequency of handling, unusual events (e.g., spills or needle sticks), and use of protective measures (e.g., personal protective equipment) that are assessed and validated to estimate exposure levels (Albertini et al. 2000; Nieuwenhuijsen 2003). Positive environmental monitoring results (e.g., surface wipes, air samples, dermal pads) provide strong evidence for the potential for occupational exposure, but they may or may not correlate to internal doses to personnel based on possible modifying variables such as an individual's use of personal protective equipment or availability of safety containment equipment, among other variables. Biomonitoring of urine or blood samples may determine the internal dose of levels of a cancer chemotherapy agent(s) or its metabolites, which identify that exposure has occurred; however, biomonitoring measurements likely provide confirmation of only the most recent exposure. A positive biomonitoring result requires that there be sufficient intake (which may be reduced by good work practices and usage of personal protective equipment) to exceed the limit of detection (LOD) for the agent tested, and the time between exposure and sample collection must be consistent with any biological processing (e.g., metabolism or excretion of the cancer chemotherapy agent). Additional limitations of monitoring studies (environmental monitoring and biomonitoring) of cancer chemotherapy agents are that they typically test only one or a handful of cancer chemotherapy agents, while multiple agents are handled, and biomonitoring studies generally evaluate only the parent drugs (e.g., cyclophosphamide) and not their metabolites. However, recent studies of environmental monitoring and biomonitoring of occupational exposure to cancer chemotherapy agents demonstrate that surface contamination continues to occur (Hon et al. 2011), and can lead to detectable internal dose levels (Hon et al. 2015). 


\section{Objectives and Research Strategy}

\section{Objectives}

The primary objective (Objective 1) of this evaluation was to undertake a systematic review to develop level-of-evidence conclusions regarding whether occupational exposure to cancer chemotherapy agents is associated with adverse health effects in humans. The types of adverse health outcomes considered are described in more detail below (see section below, PECO Statement). The secondary objective (Objective 2) was to survey the literature to determine the levels of workplace contamination by cancer chemotherapy agents and the detection of parent compound or metabolites of these agents in occupationally exposed personnel to provide context for the health outcome literature. The exposure monitoring literature was not evaluated for risk of bias as this was beyond the scope of this evaluation. As possible, environmental and biosample monitoring data from these studies were used to compare the levels reported prior to the publication of the OSHA Guidelines for Handling Hazardous Drugs in 1986 to the present time (OSHA 1986). Limitations of the systematic review were noted for Objective 1 and, for both objectives 1 and 2, the limitations of the evidence base and research needs were described.

Many commonly used cancer chemotherapy agents have already been recognized as known or probable human carcinogens by IARC (2012) and the NTP's Report on Carcinogens (NTP 2016); thus, this review did not pursue hazard identification. Instead, this review is intended to provide public health information that may be useful to workers, employers, and organizations where occupational exposure may occur to these compounds to support efforts to lower exposure. For example, genetic toxicity studies can identify the occurrence of DNA damage following exposure to occupational exposure to cancer chemotherapy agents. These data are sometimes used as surrogates for direct exposure measurements and they can also be used to provide information on potential health risks (e.g., cancer or fetal loss). This review of reproductive health outcomes associated with occupational exposure to cancer chemotherapy may also be useful to inform safety protocols for working with these agents while pregnant. The scope of this Office of Health Assessment and Translation (OHAT) evaluation is broad with the inclusion of any adverse health outcome and expands beyond the previously published reviews (Dranitsaris et al. 2005; Quansah and Jaakkola 2010), which were limited to acute effects, cancer, and/or effects on reproduction. Furthermore, the evaluation did not limit the types of exposed occupations to only medical personnel; instead, it included all reported occupational exposures to cancer chemotherapy agents (e.g., medical, manufacturing, research, veterinary, and home health care).

\section{Specific Aims}

Objective 1

- Identify literature reporting adverse health effects associated with occupational exposure to cancer chemotherapy agents.

- Extract data from relevant studies.

- Assess the internal validity (risk of bias) of individual studies assessing health outcomes.

- Summarize the extent of evidence available. 
- Synthesize the evidence for adverse health outcomes, including performance of quantitative meta-analyses if appropriate, and evaluate sources of heterogeneity.

- Rate confidence in the body of evidence for adverse health outcomes according to one of four statements: (1) high, (2) moderate, (3) low, or (4) very low/no evidence available.

- Translate confidence ratings into level of evidence for adverse health outcomes according to one of four statements: (1) high, (2) moderate, (3) low, or (4) inadequate.

- Describe limitations of the systematic review, limitations of the evidence base, and identify data gaps and key research needs.

\section{Objective 2}

- Collect and summarize the available information on levels of cancer chemotherapy agents detected in environmental monitoring studies (e.g., surface wipes, air samples of work environment) and biomonitoring (e.g., urine or blood levels) of occupationally exposed personnel.

- Describe limitations of the evidence base and identify data gaps and key research needs for exposure characterization. 


\section{Methods}

\section{Problem Formulation and Protocol Development}

Problem formulation activities and the systematic review methodology used to conduct this review were posted in a protocol in October 2015 (Appendix I). The protocol was based on: (1) deliberation with NTP staff and consultation with scientists at other federal agencies represented on the NTP Executive Committee; ${ }^{1}$ (2) discussions by the evaluation design team and other technical experts with backgrounds in occupational exposure to hazardous drugs (including cancer chemotherapy agents) and systematic review (see About This Review); (3) public review of the proposed evaluation during the April 16-18, 2014 meeting of the NTP Board of Scientific Counselors (BSC); ${ }^{2}$ (4) guidance outlined in the OHAT Handbook for Conducting a Literature-Based Health Assessment (https://ntp.niehs.nih.gov/go/systematic_review); and (5) external peer review of the draft protocol. A summary of the methods is presented below.

\section{PECO Statement}

A PECO (Population, Exposure, Comparators, and Outcomes) statement was developed as an aid to identify search terms and inclusion/exclusion criteria for Objective 1 (Table 2).

Table 2. PECO Statement for Objective 1

\begin{tabular}{cl}
\hline Element & \multicolumn{1}{c}{ Type of Evidence } \\
\hline Population & $\begin{array}{l}\text { Humans who may be exposed to cancer chemotherapy agents in their workplace, without } \\
\text { restriction based on age or sex }\end{array}$ \\
& $\begin{array}{l}\text { Exposure to cancer chemotherapy agents, including antineoplastic agents, synthetic hormones } \\
\text { (e.g., tamoxifen), biologicals, and targeted therapies (e.g., imatinib) based on indirect measures }\end{array}$ \\
& $\begin{array}{l}\text { such as job title and direct measures such as biomonitoring data (e.g., urine, blood, or other } \\
\text { specimens), when available }\end{array}$ \\
Comparators & $\begin{array}{l}\text { A comparison population exposed to lower levels (or no exposure/exposure below detection levels) } \\
\text { of cancer chemotherapy agents in their workplace }\end{array}$ \\
& $\begin{array}{l}\text { Primary outcomes: } \\
\text { Any adverse health effect (e.g., cancer, reproductive and developmental toxicity, immune system } \\
\text { effects, kidney toxicity, liver toxicity, and acute effects [i.e., alopecia, nausea]) and genetic } \\
\text { biomarkers of effect (specifically, chromosomal aberrations, micronucleus induction, and DNA } \\
\text { damage measured by the comet assay) } \\
\text { Secondary outcomes: } \\
\text { Cellular, biochemical, or clinical chemistry changes in immune system, liver, kidney, reproductive, } \\
\text { or other organ systems }\end{array}$ \\
\hline
\end{tabular}

\footnotetext{
${ }^{1}$ The NTP Executive Committee provides programmatic and policy oversight to the NTP Director and meets once or twice a year in closed forum. Members of this committee include the heads (or their designees) from the following federal agencies: Consumer Product Safety Commission (CPSC), Department of Defense (DoD), Environmental Protection Agency (EPA), Food and Drug Administration (FDA), National Cancer Institute (NCI), National Center for Environmental Health/Agency for Toxic Substances and Disease Registry (NCEH/ATSDR), National Institute of Environmental Health Sciences (NIEHS), National Institute for Occupational Safety and Health (NIOSH), Occupational Safety and Health Administration (OSHA).

${ }^{2}$ During that meeting, the BSC members considered the evaluation a high priority and no public comments were received.
} 
The NTP evaluation included cancer chemotherapy agents, such as antineoplastic agents (e.g., alkylating agents, anthracycline antibiotics, antimetabolites), hormone therapies (e.g., tamoxifen), biologicals (e.g., interferon alpha, trastuzmab), and targeted therapies (e.g., tyrosine kinase inhibitor imatinib). The evaluation did not include occupational exposure to targeted radionuclide therapy (also called radiopharmaceuticals) (Gudkov et al. 2015).

For the evaluation of adverse health effects associated with occupational exposure to cancer chemotherapy agents (Objective 1), the primary focus was on clinical diseases and disorders (e.g., cancer, spontaneous abortion, congenital malformations, abnormal functioning of the immune system, kidney, liver), and recognized acute effects observed in patients receiving cancer chemotherapy (e.g., alopecia, nausea) and genetic toxicity. Only three genetic toxicity assays were included in this evaluation because they test for a broad range of types of DNA damage induced by drugs or chemicals that induce genetic damage, including: structural chromosomal aberrations (CA), DNA damage as measured by comet assay, and micronucleus induction (structural and numerical chromosomal alterations). CA and micronucleus (MN) induction have been identified as good predictors of future cancer incidence (Bonassi et al. 2007; Bonassi et al. 2004; Norppa et al. 2006), and cancer chemotherapy agents are reported to induce CA or MN induction under experimental (e.g., in vitro or animal studies) or controlled exposure conditions (i.e., patients administered cancer chemotherapy agents for treatment of cancer) (Suspiro and Prista 2011). The comet assay is becoming more widely used in human biomonitoring to measure DNA damage as a biomarker of effect following exposure to chemicals known to induce genetic toxicity (Collins et al. 2014). However, a causal link between DNA damage detected by comet assay and subsequent induction of cancer has yet to be determined [reviewed in (Collins et al. 2014)]. The evaluation also collected information on secondary (less direct) health outcomes that are not as clearly indicative of an adverse effect, but they provide biological support and plausibility for the corresponding primary health outcomes (e.g., biochemical or clinical chemistry changes in organ systems). The evaluation did not include other biomarkers of effect (e.g., sister chromatid assay, hypoxanthine-guanine phosphoribosyltransferase [HPRT] mutations), or mechanisms of toxicity (e.g., oxidative stress, apoptosis) because there were fewer studies available, and/or their relevance to later health outcomes was less established. The evaluation also did not include studies evaluating urine mutagenesis, which is considered a biomarker of exposure.

For Objective 2, the evaluation included a literature survey of the studies reporting environmental monitoring of cancer chemotherapy agents in hospital, clinic facilities, or other occupational settings, as well as studies designed to detect the presence of cancer chemotherapy agents or their metabolites in urine or blood of personnel handling these agents or possibly exposed to such contamination. Publications that reported on levels of environmental monitoring or biomonitoring efforts were reviewed, and the data were extracted into tables (see Supplemental Files in Appendix I). These environmental monitoring and biomonitoring publications were not systematically reviewed (e.g., no risk-of-bias assessment or evaluation of confidence in the body of evidence) as that was beyond the scope of the current evaluation. The purpose of this literature survey was to gain an understanding of: (1) the frequencies and levels of positive environmental monitoring, the locations where contamination was detected, and the chemotherapy agents that were monitored and detected; (2) the extent to which worker exposures could be verified by the presence of chemotherapy agents or their metabolites in blood or urine, and (3) any possible changes in the frequency of detection or amounts of cancer chemotherapy 
agent detected in environmental monitoring/biomonitoring since implementation of the OSHA safe handling guidelines (OSHA 1986). Data on glove contamination or comparisons of the permeability of different glove materials are not included because the focus of this evaluation was on residual surface contamination, and not on these protective barriers that would be discarded following exposure. Also, studies validating new analytical techniques in the absence of measuring actual samples were also excluded from the review of environmental monitoring or biomonitoring studies.

\section{Literature Search}

The literature search was developed in collaboration with a librarian trained in systematic review methodology. Five electronic databases were searched using a search strategy tailored for each database: Embase, PubMed, Scopus, Toxline, and Web of Science. The search terms for PubMed are reported in Appendix A and the search strategy for other databases in protocol are reported in the protocol (Appendix I). No language restrictions or publication year limits were imposed, and the databases were searched on October 23, 2014 with a final updated search on February 23, 2017. The reference lists of all included studies, relevant reviews, commentaries, and other nonresearch articles as well as the NIOSH webpages detailing Occupational Exposure to Antineoplastic Agents and Other Hazardous Drugs (http://www.cdc.gov/niosh/topics/antineoplastic/default.html) were manually searched for additional relevant publications.

\section{Study Selection}

\section{Evidence Selection Criteria}

Studies were eligible for inclusion if they satisfied the eligibility criteria in the PECO statement (Objective 1) or contain relevant environmental monitoring or biomonitoring assessment information (Objective 2). Inclusion and exclusion criteria used to screen articles for relevance and eligibility at both the title-and-abstract and full-text screening stages are summarized in Table 2. The following additional exclusion criteria were applied: (1) articles without original data (e.g., editorials or reviews); (2) studies published in abstract form only (grant awards and conference abstracts); and (3) retracted articles. There were no language restrictions for the studies reporting health outcomes (Objective 1). For studies evaluating only environmental monitoring or biomonitoring (Objective 2), only studies written in English language were included because NTP was not conducting a systematic review of the exposure-only literature.

\section{Screening Process}

References retrieved from the literature search were screened for relevance and eligibility against the evidence selection criteria using DistillerSR ${ }^{\circledR}$ (Evidence Partners; https://www.evidencepartners.com/products/distillersr-systematic-review-software/), a database software program. Screeners from the evaluation team were trained with an initial pilot phase undertaken to improve clarity of the evidence selection criteria and to improve accuracy and consistency among screeners. Two trained screeners (one of whom was the project lead) independently screened the references at the title-and-abstract level to determine whether a reference met the evidence selection criteria. References that were not excluded by reviewing the title and abstract were screened by a full-text review, including references that were considered 
of unclear relevance based on the title-and-abstract review. Screening conflicts were resolved through discussion. Following full-text review, the remaining studies were "included" and used for the evaluation.

\section{Data Extraction}

\section{Extraction Process}

Data were collected (i.e., extracted) from included studies by one member of the evaluation team and checked by a second member for completeness and accuracy. Any discrepancies in the data extraction were resolved by discussion. Information that was inferred, converted, or estimated during data extraction is annotated, e.g., using brackets [ $n=10]$. Authors were contacted when details were missing on key study findings. The data extraction files note if an attempt was made to contact study authors by email for missing data considered important for evaluating key study findings, and whether a response from the author was received.

\section{Data Availability}

Data extraction was completed using the Health Assessment Workspace Collaborative (HAWC), an open-source and freely available web-based interface application, for visualization and warehousing. ${ }^{3}$ The data extraction results for included studies are publicly available (https://hawcproject.org/assessment/196/) and can be downloaded in Excel format through HAWC (NTP 2015a). Data extraction elements are listed separately for human, animal, and in vitro studies in the protocol (Appendix I) (NTP 2015b).

\section{Quality Assessment of Individual Studies}

Risk of bias was assessed for individual studies using a tool developed by OHAT (https://ntp.niehs.nih.gov/go/systematic_review); the risk-of-bias criteria were customized for the evaluation (e.g., to address occupational exposure scenarios to cancer chemotherapy agents). The OHAT risk-of-bias tool has questions tailored to specific study design types to assess risk of bias in six categories, including selection bias, confounding bias, performance bias, attrition bias, detection bias, and selective reporting (Rooney et al. 2014). Studies were independently evaluated by two trained assessors who answered all applicable risk-of-bias questions with one of four options (Figure 1) following pre-specified criteria. The criteria describe aspects of study design, conduct, and reporting required to reach risk-of-bias ratings for each question and specify factors that can distinguish among ratings (e.g., what separates "definitely low" from "probably low” risk of bias). Risk-of-bias assessments for confounding, exposure characterization, and outcome assessment were considered especially critical.

\footnotetext{
${ }^{3}$ HAWC ( $\underline{\text { Health }}$ Assessment Workspace Collaborative): A Modular Web-based Interface to Facilitate Development of Human Health Assessments of Chemicals (https://hawcproject.org/portal/).
} 


\begin{tabular}{ll}
\hline Symbol & \multicolumn{1}{c}{ Description } \\
\hline++ & $\begin{array}{l}\text { Definitely Low risk of bias: } \\
\text { There is direct evidence of low risk of bias practices. }\end{array}$ \\
\hline$+\quad \begin{array}{l}\text { Probably Low risk of bias: } \\
\text { There is indirect evidence of low risk of bias practices OR it is deemed that deviations from low risk of } \\
\text { bias practices for these criteria during the study would not appreciably bias results, including } \\
\text { consideration of direction and magnitude of bias. }\end{array}$ \\
\hline$-\quad \begin{array}{l}\text { Probably High risk of bias: } \\
\text { There is indirect evidence of high risk of bias practices (indicated with “-”) } \\
\text { OR there is insufficient information provided about relevant risk of bias practices (indicated as not } \\
\text { reported (NR)). Both symbols indicate probably high risk of bias. }\end{array}$ \\
\hline$-\quad \begin{array}{l}\text { Definitely High risk of bias: } \\
\text { There is direct evidence of high risk of bias practices. }\end{array}$ \\
\hline
\end{tabular}

Figure 1. Risk-of-bias Assessment Ratings

Answers to the risk-of-bias (study quality) questions results in one of four risk-of-bias ratings.

Any discrepancies in ratings between assessors were resolved through discussion to reach the final recorded risk-of-bias rating for each question along with a statement of the basis for that rating. Members of the evaluation team were consulted for assistance if additional expertise was necessary to reach final risk-of-bias ratings based on specific aspects of study design or performance reported for individual studies. Information or study procedures that were not reported were assumed not to have been conducted, resulting in an assessment of "probably high" risk of bias. NTP queried authors by email to obtain missing information, and responses received were used to update risk-of-bias ratings.

The OHAT method identifies some risk-of-bias questions as more important than others because there is evidence that these areas of bias generally have a larger impact on the credibility of study results in environmental health literature (Rooney et al. 2016). The key questions for observational human studies are: confounding, exposure characterization, and outcome assessment (including blinding of outcome assessors).

\section{Rating Confidence in Bodies of Evidence}

Groups or studies (bodies of evidence) were assessed to develop confidence ratings that reflect the certainty in the evidence that occupational exposure to chemotherapy agents are associated with health effects for each health outcome using the GRADE system as adapted by OHAT (Rooney et al. 2014). Thus, for each health outcome, bodies of evidence were assessed to develop confidence ratings that reflect the certainty in the evidence that occupational exposure to cancer chemotherapy agents are associated with health effects. More detailed guidance on reaching confidence ratings in the body of evidence as "high," "moderate," "low," or "very low" is provided in the OHAT Handbook for Conducting a Literature-Based Health Assessment (https://ntp.niehs.nih.gov/go/systematic_review, see STEP 5). In brief, available studies on an outcome were initially grouped by key study design features, and each grouping of studies was given an initial confidence rating by those features (Column 1 of Figure 2), including controlled exposure, exposure prior to outcome, individual outcome data, and comparison group used. For example, cohort studies (either prospective or retrospective) are generally assigned an initial 
confidence rating of "moderate" because they have three factors (exposure prior to outcome, individual outcome data, and comparison group). Cross-sectional studies are, generally, assigned an initial confidence rating of "low" because they only have two factors (individual data and comparison group). The cross-sectional study design generally cannot assure that exposure occurred prior to outcome; therefore, there is decreased confidence that the relationship between exposure and outcome is causal. Concerns about possible reverse causation or the potential influence of the outcome on the exposure can limit confidence in the results of cross-sectional studies. However, there are several factors that could increase the confidence that data from cross-sectional studies more strongly supports the causal association between exposure and outcome and, thus, increase the initial confidence rating to "moderate." Two examples of factors that could increase the confidence in data from cross-sectional studies are (1) the outcome was rapid (e.g., occurring within minutes or hours of exposure) (see section below, Adjustment of Initial Confidence Ratings of Cross-sectional Studies Evaluating Genetic Toxicity), and (2) the exposure was persistent (e.g., exposure would have preceded outcome even in a cross-sectional study design).

Potential downgrading of the confidence rating was considered for factors that decrease confidence in the results (Column 2 of Figure 2, [high risk of bias, unexplained inconsistency, indirectness or lack of applicability, imprecision, and publication bias]), and potential upgrading of the confidence rating was considered for factors that increase confidence in the results (Column 3 of Figure 2, [large magnitude of effect, dose-response, consistency across study designs/populations, consideration of residual confounding, and other factors that increase confidence in the association or effect]). Consideration of consistency across study designs or human populations is not included in the GRADE guidance (Guyatt et al. 2011); however, it is considered in the modified version of GRADE used by OHAT (Rooney et al. 2014).

Member of the evaluation review team made the confidence ratings assessments. Any discrepancies between reviewers were resolved by consensus and by consultation with technical advisors, as needed. Confidence ratings are summarized in evidence profile tables for each outcome.

\section{Adjustment of Initial Confidence Ratings of Cross-sectional Studies Evaluating Genetic Toxicity}

For the evaluation of the three genetic toxicity assays, cross-sectional studies were assigned an initial confidence rating of "moderate" because there was compelling evidence to conclude that occupational exposure preceded outcome and reverse causation was highly unlikely. It is highly likely that the cross-sectional study design ensures that occupational exposure to the cancer chemotherapy agent preceded the health outcome for genetic toxicity assays based on three factors: (1) the rapid induction of DNA damage, (2) inclusion in most studies of only subjects who worked full time the week that their blood samples were drawn, and (3) an employment duration ranging from at least a month to more than one year at the time the study was conducted. Due to the rapid induction of genetic damage, for studies designed to measure DNA damage using the comet assay, it is recommended that blood samples be collected within 3 to 4 hours of exposure termination. For studies designed to measure induction of chromosomal damage or micronuclei, it is recommended that blood samples be obtained within 2 days of acute exposure (Albertini et al. 2000). Furthermore, reverse causation is unlikely in the cross-sectional 
studies of genetic toxicity as participants are not likely to know their DNA damage status such that it would influence their response to a survey of exposures.

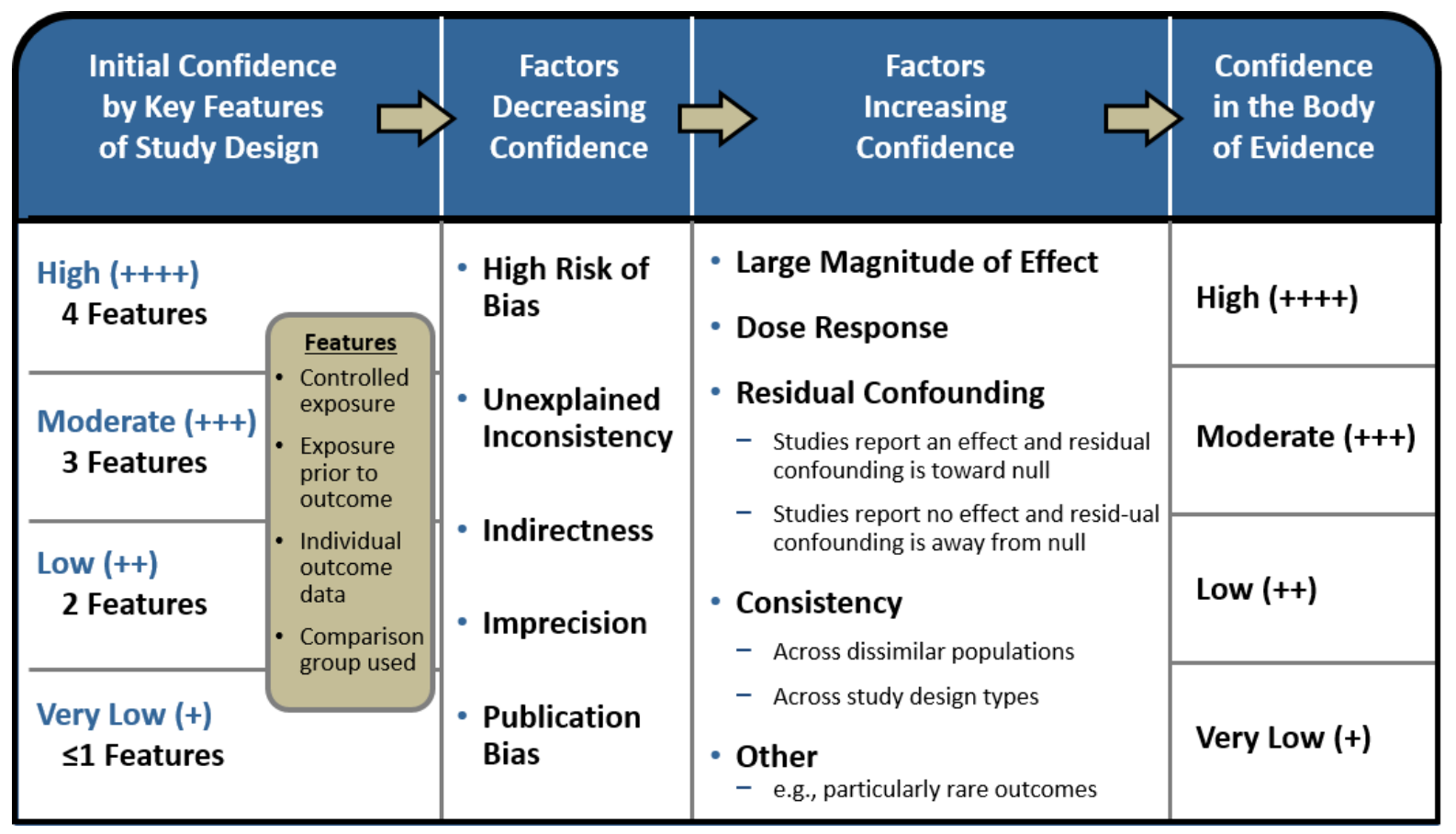

Figure 2. Assessing Confidence in the Body of Evidence

This figure describes how confidence in bodies of evidence (i.e., groups of studies) are considered on an outcome basis.

\section{Preparation of Level-of-evidence Conclusions}

The confidence ratings were translated into level-of-evidence conclusions that reflect the confidence in the bodies of evidence and the direction of effect (i.e., health effect or no health effect). These conclusions indicate the confidence in the available evidence that occupational exposure to chemotherapy agents are associated with health effects for each type of health outcome, separately, according to one of four statements: (1) high, (2) moderate, (3) low, or (4) inadequate (Figure 3 and Table 3).

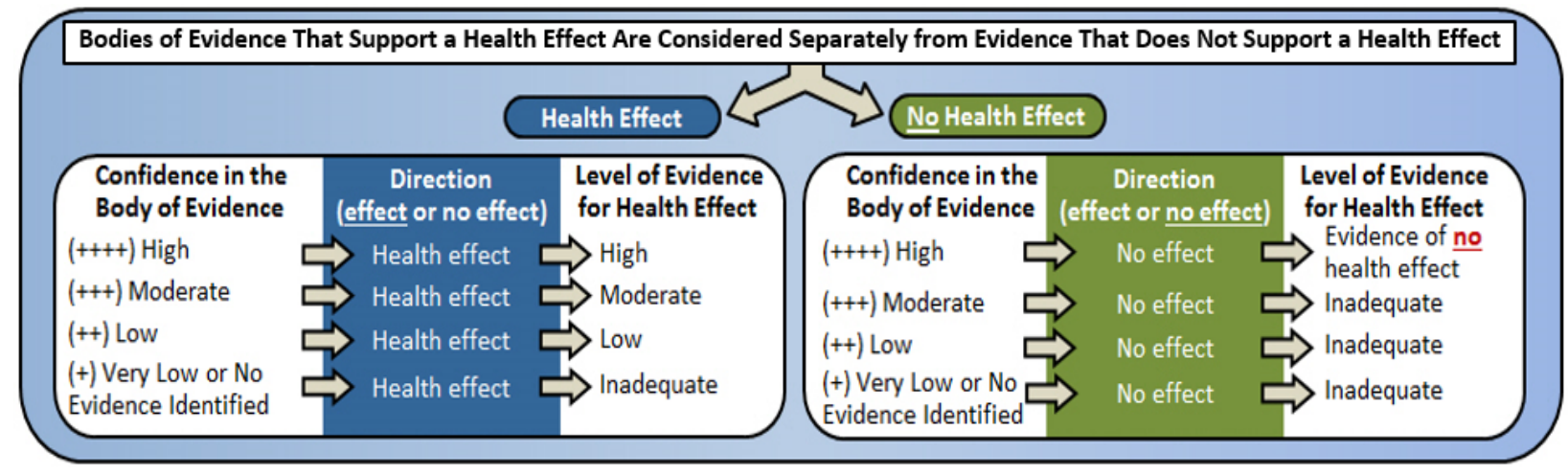

Figure 3. Translation of Confidence Ratings into Evidence of Health Effect Conclusions 
Systematic Review of Occupational Exposure to Cancer Chemotherapy Agents

Table 3. Definitions of Level-of-evidence Descriptors

\begin{tabular}{ll}
\hline \multicolumn{1}{c}{ Evidence Descriptors } & \multicolumn{1}{c}{ Definition } \\
\hline High Level of Evidence & $\begin{array}{l}\text { There is high confidence in the body of evidence for an association between } \\
\text { occupational exposure to cancer chemotherapy agents and the health outcome(s). } \\
\text { Moderate Level of Evidence }\end{array}$ \\
Low Level of Evidence & $\begin{array}{l}\text { Ther is moderate confidence in the body of evidence for an association between } \\
\text { occupational exposure to cancer chemotherapy agents and the health outcome(s). } \\
\text { There is low confidence in the body of evidence for an association between } \\
\text { occupational exposure to cancer chemotherapy agents and the health outcome(s), } \\
\text { or no data are available. }\end{array}$ \\
Inadequate Evidence & $\begin{array}{l}\text { There is insufficient evidence available to assess if occupational exposure to } \\
\text { cancer chemotherapy agents is associated with the health outcome(s). }\end{array}$ \\
Evidence of No Health Effect & $\begin{array}{l}\text { There is high confidence in the body of evidence that occupational exposure to } \\
\text { cancer chemotherapy agents is not associated with the health outcome(s). }\end{array}$ \\
\hline
\end{tabular}




\section{Results and Evidence Synthesis}

\section{Literature Search Results}

The electronic database searches retrieved 5,584 unique references, and an additional 58 references were identified by experts and by reviewing published reviews and reference lists of included studies. From the total references retrieved: 4,433 were excluded during the title-andabstract screening and 953 were excluded during the full-text review. The screening results are outlined in a study selection diagram with reasons for exclusion documented at the full-text review (Figure 4). A total of 110 studies reported primary or secondary health outcomes, which were data extracted and processed for risk-of-bias (Objective 1); the included health studies are listed in Appendix B, including 12 non-English language studies that were professionally translated to English language. A total number of 171 studies evaluated contamination of the workplace (environmental monitoring) or biomonitoring of personnel handling cancer chemotherapy agents (Objective 2), including 18 studies that reported on both environmental monitoring and biomonitoring.

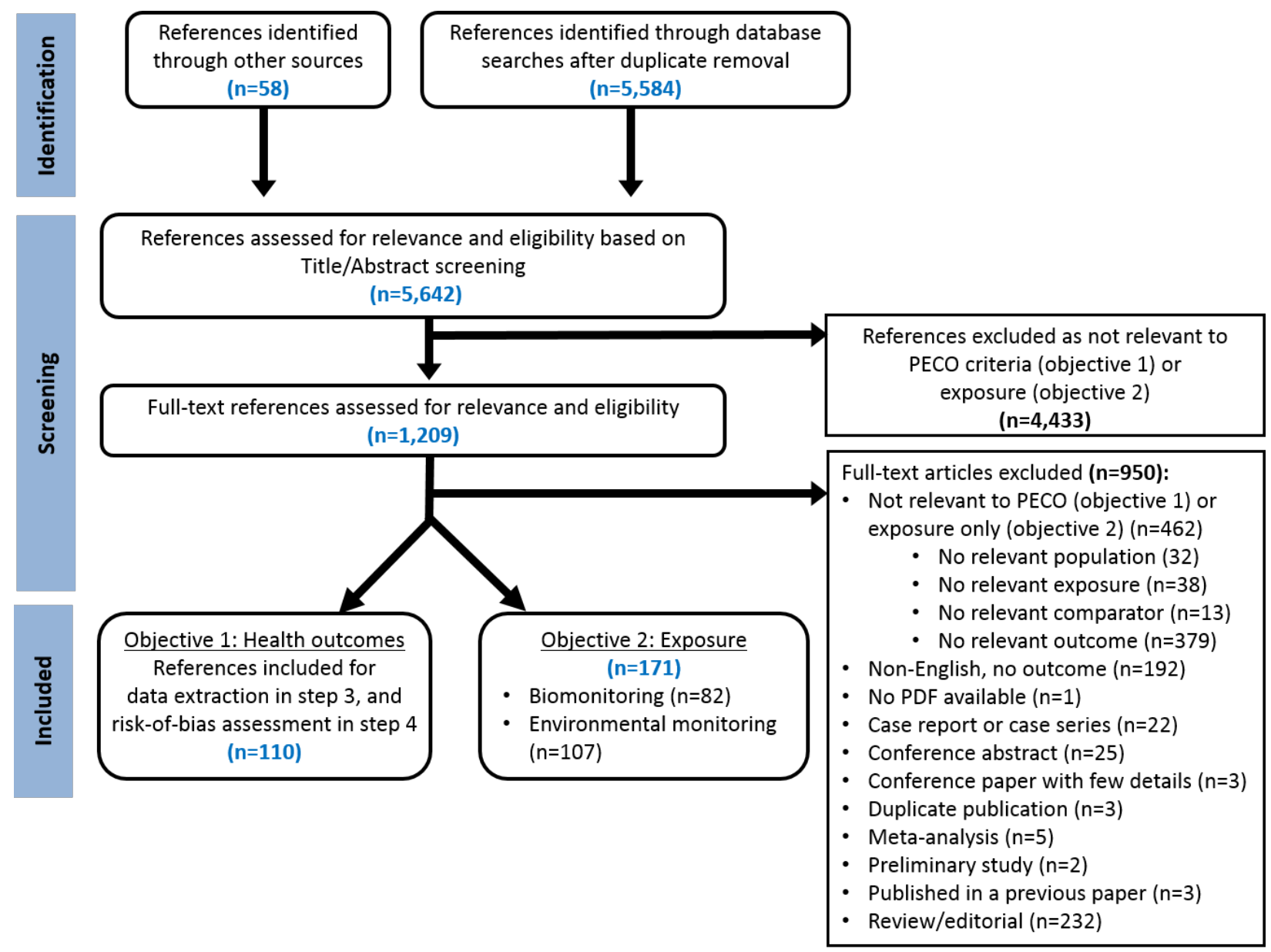

Figure 4. Study Selection Diagram

The total number of included studies was 251, including 110 studies reporting on health outcomes. Seventeen studies on health outcome also reported on biomonitoring $(n=7)$, environmental monitoring $(n=3)$, or both $(n=7)$. Eighteen exposure studies reported on both biomonitoring and environmental monitoring. 
Twenty-one studies were excluded from the body of evidence of health effects in reaching health effects conclusions because of methodological considerations and the relatively large body of evidence that reported data using preferred methods. Studies were excluded from the evidence synthesis step because they did not use the preferred measurements for genetic toxicity, including: the inclusion of gaps for CA (Goloni-Bertollo et al. 1992; Mahrous et al. 1998), and the number of binucleated lymphocytes with one, two, three or four micronuclei without analyzing the total binucleated lymphocytes with micronuclei (Fucic et al. 1998). Eight studies, including Fucic et al. (1998), reporting on CA were excluded because they only reported the number of individual types of CA per 100 cells, instead of the total number of CA per 100 cells (Benhamou et al. 1988; Boughattas et al. 2010; Fucic et al. 1998; Harris et al. 1992; Oestreicher et al. 1990; Sarto et al. 1990; Stiller et al. 1983; Waksvik et al. 1981). While the OECD protocol recommends reporting of the frequency of individual CA (OECD 2016a), these studies did not consistently report on the same types of individual CA or grouped data (e.g., chromosome breaks, chromatid breaks), which made it difficult to compare between the studies. Six studies were excluded because they included preliminary data (Maluf and Erdtmann 2000a; Villarini et al. 2012) or they included data that were published in prior publications. For example, Cavallo et al. $(2007$; 2009) were excluded because they evaluated the effect of gene polymorphisms on micronuclei and comet assay data published in a prior publications (Cavallo et al. 2005; Ursini et al. 2006), and two studies reporting on number of CA per 100 cells scored (Mušák et al. 2009a; Mušák et al. 2009b) were excluded because the data were published in (Mušák et al. 2006). One study was excluded because it reported on combined, instead of individual outcomes: adverse pregnancy outcomes combined, which included spontaneous abortion, stillbirth and congenital malformations (Rogers and Emmett 1987). Finally, four studies reporting on the percentage of cells with CA of the same population of nurses in Hungary were excluded due to a lack of adjustment for significant effects of co-exposure to ionizing radiation on exposed subjects, which made it unclear whether the significant finding of higher percentage of cells with CA was due to occupational exposure to cancer chemotherapy agents or to the co-exposed individuals (Tompa et al. 2016; Tompa et al. 2006a; Tompa et al. 2015; Tompa et al. 2006b).

The main findings for each category of health outcome are summarized below, and the number of studies per health outcome are reported in Table 4. The results and evidence synthesis sections include a detailed analysis of the evidence on spontaneous abortion and genetic toxicity. The risk-of-bias assessment of individual studies and for the body of evidence were considered in developing the confidence ratings for each health effect. The key risk-of-bias questions were: confounding, exposure characterization, and outcome assessment (see protocol in Appendix I); they are discussed in the consideration of the body of evidence for each health effect. While no study was excluded based on concerns for risk of bias, confidence conclusions were considered with and without the high risk-of-bias studies (e.g., studies rating probably high or definitively high risk of bias for two key risk-of-bias questions) to assess the influence of the high risk-ofbias studies. The risk-of-bias assessment for all included studies is in Appendix C. 
Table 4. Categories of Health Outcomes for 110 Included Studies

\begin{tabular}{|c|c|c|c|c|c|c|c|c|}
\hline $\begin{array}{c}\text { Health } \\
\text { Outcome }^{\mathrm{a}}\end{array}$ & $\begin{array}{c}\text { DNA } \\
\text { Damage }^{b}\end{array}$ & $\begin{array}{c}\text { Reproduc- } \\
\text { tive and } \\
\text { Develop- } \\
\text { mental } \\
\text { Toxicity }\end{array}$ & $\begin{array}{c}\text { Immune } \\
\text { System } \\
\text { Alterations }\end{array}$ & $\begin{array}{l}\text { Acute } \\
\text { Effects }\end{array}$ & $\begin{array}{l}\text { Kidney } \\
\text { Toxicity }\end{array}$ & $\begin{array}{c}\text { Liver } \\
\text { Toxicity }\end{array}$ & Cancer & $\begin{array}{l}\text { Cardiac } \\
\text { System } \\
\text { Toxicity }\end{array}$ \\
\hline $\begin{array}{l}\text { Primary } \\
\text { (Direct) }\end{array}$ & $\begin{array}{l}66 \\
\text { studies }^{\mathrm{b}}\end{array}$ & $\begin{array}{l}30 \text { studies } \\
\text { (e.g., } \\
\text { spontaneous } \\
\text { abortion) }\end{array}$ & $\begin{array}{l}1 \text { study (e.g., } \\
\text { immune- } \\
\text { related } \\
\text { diseases) }\end{array}$ & $\begin{array}{l}10 \text { studies } \\
\text { (e.g., } \\
\text { nausea) }\end{array}$ & $\begin{array}{l}1 \text { study (e.g., } \\
\text { kidney } \\
\text { disease) }\end{array}$ & $\begin{array}{l}1 \text { study (e.g., } \\
\text { liver disease) }\end{array}$ & 3 studies & $\begin{array}{l}1 \text { study (e.g., } \\
\text { cardiovas- } \\
\text { cular } \\
\text { disease) }\end{array}$ \\
\hline $\begin{array}{l}\text { Secondary } \\
\text { (Indirect) }\end{array}$ & - & - & $\begin{array}{l}10 \text { studies } \\
\text { (e.g., } \\
\text { lymphocyte } \\
\text { activation) }\end{array}$ & - & $\begin{array}{l}4 \text { studies } \\
\text { (e.g., kidney } \\
\text { biochemistry) }\end{array}$ & $\begin{array}{l}3 \text { studies } \\
\text { (e.g., liver } \\
\text { biochemistry) }\end{array}$ & - & $\begin{array}{l}1 \text { study (e.g., } \\
\text { cardiac } \\
\text { functional } \\
\text { endpoints) }\end{array}$ \\
\hline
\end{tabular}

andividual studies often reported more than one health outcome.

bDNA damage studies included only those evaluating chromosomal aberrations $(n=29)$, micronucleus induction $(n=35)$, and DNA damage measured by the comet assay $(n=21)$.

\section{Health Outcomes with Inadequate Evidence}

For many of the health outcomes, there is inadequate evidence to determine whether there is an association with occupational exposure to cancer chemotherapy. The published studies were considered inadequate to evaluate potential reproductive or development toxicity on endpoints other than spontaneous abortion because most of the studies had a small number of cases for each health outcome, and some outcomes had very few studies. These outcomes included: cancer, congenital malformations, menstrual irregularities, ectopic pregnancy, stillbirth, preterm birth, low birth weight/small for gestational age, sex ratio, and time to pregnancy or infertility. The body of evidence was inadequate to evaluate potential effects on the immune system (e.g., hematotoxicity) because there was heterogeneity in the endpoints examined and, when evaluated in more than one study, the results were inconsistent. The body of evidence was also inadequate for liver or kidney toxicity because these health effects were only reported in one or two studies with inconsistent results. In addition, the number of cases were low (e.g., $n=3-6$ cases out of 44 exposed medical personnel for liver or kidney toxicity, respectively), and there was considerable heterogeneity in the endpoints examined. The results, evidence synthesis and risk-of-bias assessment for the health outcomes with inadequate evidence are in Appendix E.

\section{Spontaneous Abortion}

Spontaneous abortion (also called miscarriage) is the noninduced termination of a pregnancy before 20 weeks of gestation. NTP identified 16 studies reporting on spontaneous abortion in women or spouses of men occupationally exposed to cancer chemotherapy agents. Among the studies in this evaluation, spontaneous abortion was most commonly defined as spontaneous pregnancy loss at $\leq 20$ weeks of gestation; however, one study defined spontaneous abortion as pregnancy loss at less than 24 weeks of gestation (Zhang et al. 2016) and two studies defined it as pregnancy loss at less than 28 weeks of gestation (McDonald et al. 1988b; Stücker et al. 1990). The level-of-evidence conclusion is based only on studies that reported spontaneous abortions, and did not include studies that reported spontaneous abortion and stillbirth as a combined outcome (McAbee et al. 1993; Rogers and Emmett 1987). 


\section{Summary}

Based on the available studies, there is moderate confidence in the body of evidence that occupational exposure to cancer chemotherapy agents is associated with spontaneous abortion. Seven of the 16 studies identified were considered to be more informative regarding exposure assessment because the studies identified exposed nursing or pharmacy staff based on the task they performed (e.g., prepared and administered cancer chemotherapy agents) and, in some studies, the frequency of handling these agents (e.g., number of times hourly or weekly) (Fransman et al. 2007c; Lawson et al. 2012; Peelen et al. 1999; Selevan et al. 1985; Stücker et al. 1990; Valanis et al. 1999; Zhang et al. 2016). The body of evidence from these seven studies was used for rating confidence in the conclusions because of their exposure characterization and concerns for high risk of bias for exposure characterization as well as other key risk-of-bias factors (confounding and outcome assessment) in the remaining nine studies. The results were inconsistent for the nine studies that evaluated occupational exposure to any chemical or work stressor (e.g., shift work, heavy lifting) (Hemminki et al. 1985; McDonald et al. 1988b; Schaumburg and Olsen 1990b; Taskinen et al. 1994; Taskinen et al. 1986) or risk-of-bias issues regarding exposure characterization (Skov et al. 1992; Walusiak et al. 2003) and/or outcome assessment (Elshamy et al. 2010; Medková 1991a). There were no changes from the initial rating of moderate confidence in the body of evidence (seven studies) after considering factors that may increase or decrease confidence (Figure 5). The final rating of moderate confidence in the body of evidence translates directly into a moderate level of evidence that occupational exposure to cancer chemotherapy agents is associated with a higher risk of spontaneous abortion.

\section{Overview}

The available 16 epidemiological studies in the body of evidence that evaluated the association between occupational exposure to cancer chemotherapy and spontaneous abortions included four retrospective cohort studies, four case-control studies, and eight cross-sectional studies of 13 unique study populations (Table 5). While all studies evaluated the pregnancy outcomes of occupationally exposed female subjects, two studies also evaluated spontaneous abortion among female spouses of occupationally exposed male subjects (Valanis et al. 1999; Walusiak et al. 2003). The majority of the studies evaluated nurses, one study evaluated pharmaceutical employees (Taskinen et al. 1986), and one evaluated laboratory workers (Taskinen et al. 1994). The studies were conducted in 11 countries: Denmark (two studies), Finland (four studies; two studies overlapped in population being evaluated), the Netherlands (two studies evaluated the same population and same data), the United States (two studies), and one study each from Canada, China, Czech Republic, Egypt, France, and Poland. All the studies used retrospective exposure characterization that relied primarily on job title and self-reported questionnaires with questions about work tasks, exposures, and use of safety equipment (e.g., biological safety cabinets and personal protective equipment during or prior to pregnancy). Two studies evaluating the same study population also reported on biomonitoring or environmental monitoring: swipe tests for cyclophosphamide, ifosfamide, 5-flurouracil and urine biomonitoring for cyclophosphamide (Peelen et al. 1999), and glove and hand wash sampling for cyclophosphamide (Fransman et al. 2007c). The occurrence of spontaneous abortion was identified from medical records (five studies) or via questionnaire (interviewer-led questionnaire, four studies; self-reported questionnaire, six studies). Data collection on the study participants ranged from the years of 1970 to 2013: five studies (four unique populations) reported data prior to 1985, two studies overlapped with 1985 U.S. guidance on handling hazardous drugs, and eight 
studies (six unique populations) reporting data collected after 1985. Six of these studies also reported data on other reproductive outcomes, cancer, and/or acute effects (see Appendix E for discussion of the other health outcomes).

\section{Evidence Synthesis}

The evidence synthesis for spontaneous abortion was based on 16 studies, including: four retrospective cohort studies (Lawson et al. 2012; McDonald et al. 1988b; Schaumburg and Olsen 1990b; Skov et al. 1992), four case-control studies (Hemminki et al. 1985; Selevan et al. 1985; Taskinen et al. 1994; Taskinen et al. 1986), and eight cross-sectional studies (Elshamy et al. 2010; Fransman et al. 2007c; Medková 1991a; Peelen et al. 1999; Stücker et al. 1990; Valanis et al. 1999; Walusiak et al. 2003; Zhang et al. 2016) (Table 5). The results were consistent for a higher risk of spontaneous abortions associated with cancer chemotherapy agents when evaluating studies of nursing or pharmacy personnel with higher handling activities of these agents (Figure D-1) (Fransman et al. 2007c; Lawson et al. 2012; Peelen et al. 1999; Selevan et al. 1985; Stücker et al. 1990; Valanis et al. 1999). For example, a case-control study of Finnish nurses reported significantly greater odds of spontaneous abortion $(\mathrm{OR}=2.3,95 \% \mathrm{CI}=1.2-$ 4.39) in nurses with high usage of cancer chemotherapy drugs compared to nurses with less than once weekly exposure in the first trimester (Selevan et al. 1985). A cross-sectional study in France by Stucker et al. (1990) reported significantly higher odds of spontaneous abortion (adjusted odds ratio $($ adjOR $)=1.7,95 \% \mathrm{CI}=1.0-2.8)$ in exposed nurses employed in a cancer center or hospital oncology wards compared to unexposed nurses from consulting medical, cardiology, endocrinology, and general medical units. Similarly, a cross-sectional study of female nurses, nurses' aides, pharmacists, and pharmacy technicians in the United States reported significantly increased odds of spontaneous abortion (adjOR $=1.5,95 \% \mathrm{CI}=1.2-1.8$ ) in medical personnel who either prepared or administered doses, and/or handled the excreta of patients administered cancer chemotherapy agents during pregnancy than unexposed subjects (Valanis et al. 1999). In a study evaluating the influence of occupational exposure in the Nurses' Health Study II in the United States, Lawson et al. (2012) reported significantly greater odds of spontaneous abortion in nurses exposed $\geq 1$ hour per day to cancer chemotherapy agents (adjOR=1.94, 95\% CI = 1.32-2.86), with the highest odds of spontaneous abortion occurring at $<12$ weeks of gestation; the analyses were adjusted for other co-exposures and work conditions (i.e., anesthetic gases, sterilizing agents, anti-viral agents, ionizing radiation, and shift work).

Further support for a relationship between occupational exposure to cancer chemotherapy agents and spontaneous abortion was reported in a cross-sectional study of nurses handling cancer chemotherapy agents employed in an oncology ward and non-oncology ward compared to unexposed nurses in a hospital in China at the beginning of the implementation of a pharmacy intravenous admixture service (PIVAS) versus an open plan treatment area, with a follow-up study 2 years later (Zhang et al. 2016). The PIVAS was a closed system that reduced exposure by adding safety equipment (e.g., biological safety cabinets, sealed bags for transport of prepared drugs), increased use of personal protective equipment when handling these agents, and protocols for storage, transport, disposal, and management of spills as well as safety training. Prior to implementation of the PIVAS, the rate of spontaneous abortions was non-significantly higher in nurses handling cancer chemotherapy in oncology (14.6\%) compared to unexposed nurses (9.4\%). In a follow-up study 2 years later, the authors observed a significant reduction in the rate of spontaneous abortions in nurses working in the oncology ward 2 years after PIVAS implementation (4.6\%) compared to the rates before PIVAS (14.6\%), as well as compared to 
spontaneous abortion rates in oncology nurses from a hospital in China with no PIVAS (22.3\%) (Zhang et al. 2016).

In contrast, two studies of one population observed no association of spontaneous abortion (Fransman et al. 2007c; Peelen et al. 1999). Peelen et al. (1999) reported a slightly higher, but not statistically significant, odds of spontaneous abortion in oncology nurses from 121 hospitals in the Netherlands based on job title and work tasks (OR $=1.4,95 \% \mathrm{CI}=0.8-2.6)$. In a second analysis of the same data from the Dutch oncology nurses, Fransman et al. (2007c) reported no association of spontaneous abortions with estimated dermal exposure to cyclophosphamide; the dermal exposure estimate was based on job title tasks, use of PPE as well as glove and hand contamination. The author commented that the study may have underestimated exposure of the nurses because the administered dose levels of cancer chemotherapy agents have decreased over time, and the outcome data were collected in 1990-1997, while the glove and hand monitoring studies were conducted in 1996-1997 and 2001-2003. 
Table 5. Studies Reporting on Spontaneous Abortion

\begin{tabular}{|c|c|c|c|c|c|c|}
\hline Study & $\begin{array}{c}\text { Study Design } \\
\text { (Occupation, Location, } \\
\text { Years Data Collected, } \\
\text { Sex) }\end{array}$ & $\begin{array}{c}\text { No. of Pregnancies } \\
\text { (Unless Otherwise } \\
\text { Stated) }\end{array}$ & Exposure Measure & $\begin{array}{l}\text { Outcome } \\
\text { Assessment }\end{array}$ & Analysis & Results \\
\hline $\begin{array}{l}\text { Elshamy et al. } \\
\text { (2010) }\end{array}$ & $\begin{array}{l}\text { Cross-sectional (Nurses, } \\
\text { Egypt, 2006, F) }\end{array}$ & $\begin{array}{l}35 \text { exposed, } 29 \\
\text { unexposed }\end{array}$ & $\begin{array}{l}\text { Job title, length of } \\
\text { employment, self- } \\
\text { administered questionnaire } \\
\text { about drug handling, } \\
\text { frequency of handling, } \\
\text { number of accidents with } \\
\text { cancer chemotherapy in } \\
\text { past year, usage of } \\
\text { personal protective } \\
\text { equipment, and training. } \\
\text { Note: Only } 42.9 \% \text { of } \\
\text { nurses reported using } \\
\text { gloves when handling } \\
\text { patient waste, and } 45.7 \% \\
\text { of nurses admitted to } \\
\text { eating in the drug } \\
\text { preparation area }\end{array}$ & $\begin{array}{l}\text { Self-reported } \\
\text { questionnaire; no } \\
\text { definition of abortions } \\
\text { was provided [assumed } \\
\text { it was spontaneous } \\
\text { abortion] }\end{array}$ & $\begin{array}{l}\text { Chi-square test } \\
\text { with continuity } \\
\text { correction, if } \\
\text { indicated }\end{array}$ & $\begin{array}{l}\text { Significantly }(\mathrm{p}<0.05) \\
\text { higher rates of } \\
\text { spontaneous abortions } \\
\text { in exposed }(22.6 \% \text {; } 8 \\
\text { cases) versus unexposed } \\
(10.3 \% \text {; } 3 \text { cases) }\end{array}$ \\
\hline $\begin{array}{l}\text { Fransman et al. } \\
(2007 c)\end{array}$ & $\begin{array}{l}\text { Cross-sectional (Nurses, the } \\
\text { Netherlands, 1990-1997, F) } \\
\text { Note: Same data as Peelen } \\
1997\end{array}$ & $\begin{array}{l}\text { Exposed: } 279 \\
\text { background, } 146 \text { low, } \\
145 \text { medium, } 134 \text { high; } \\
555 \text { unexposed }\end{array}$ & $\begin{array}{l}\text { Estimated dermal } \\
\text { exposure based on job } \\
\text { title, self-reported } \\
\text { questionnaire, and glove } \\
\text { usage in first month of } \\
\text { pregnancy (or during time } \\
\text { trying to get pregnant), } \\
\text { combined with data on CP } \\
\text { glove monitoring } \\
\text { conducted in } 1996-1997 \\
\text { and glove and hand } \\
\text { monitoring in } 2003-2007 \text {; } \\
\text { categorized as: unexposed, } \\
\text { background, low } \\
\text { ( } \leq 0.20 \mu \mathrm{g} \mathrm{CP} / \mathrm{wk}) \text {, } \\
\text { medium }(>0.20-0.74 \mu \mathrm{g} \\
\mathrm{CP} / \mathrm{wk}) \text { or high exposure } \\
(>0.74 \mu \mathrm{g} \mathrm{CP} / \mathrm{wk})\end{array}$ & $\begin{array}{l}\text { Self-reported } \\
\text { questionnaire on most } \\
\text { recent pregnancy; } \\
\text { defined as pregnancy } \\
\text { loss at <20 weeks of } \\
\text { gestation }\end{array}$ & $\begin{array}{l}\text { Logistic regression } \\
\text { analysis; adjusted a } \\
\text { priori for age at } \\
\text { conception, parity, } \\
\text { smoking, alcohol } \\
\text { consumption, } \\
\text { coffee intake } \\
\text { during 1st trimester } \\
\text { of pregnancy, } \\
\text { vitamin/folic acid } \\
\text { use during } \\
\text { pregnancy as well } \\
\text { as work factors } \\
\text { (e.g., co- } \\
\text { exposures) }\end{array}$ & $\begin{array}{l}\text { No difference in odds of } \\
\text { spontaneous abortions } \\
\text { compared to unexposed } \\
\text { pregnancies (log-linear } \\
\text { association OR = 1.01, } \\
\text { 95\% CI = 0.93-1.10; } 36 \\
\text { exposed cases). Using } \\
\text { dermal exposure } \\
\text { estimates, the odds of } \\
\text { spontaneous abortion } \\
\text { for exposed pregnancies } \\
\text { were: low exposure, } \\
\text { adjOR = 1.2 (95\% } \\
\text { CI = 0.6-2.7); medium } \\
\text { exposure, adjOR = 0.8 } \\
\text { (95\% CI = 0.3-2.0); and } \\
\text { high exposure, } \\
\text { adjOR = 1.2 (95\% } \\
\text { CI 0.6-2.7) }\end{array}$ \\
\hline
\end{tabular}




\begin{tabular}{|c|c|c|c|c|c|c|}
\hline Study & $\begin{array}{c}\text { Study Design } \\
\text { (Occupation, Location, } \\
\text { Years Data Collected, } \\
\text { Sex) }\end{array}$ & $\begin{array}{c}\text { No. of Pregnancies } \\
\text { (Unless Otherwise } \\
\text { Stated) }\end{array}$ & Exposure Measure & $\begin{array}{c}\text { Outcome } \\
\text { Assessment }\end{array}$ & Analysis & Results \\
\hline $\begin{array}{l}\text { Hemminki et al. } \\
\text { (1985) }\end{array}$ & $\begin{array}{l}\text { Case-control (Nurses, 1973- } \\
\text { 1979, Finland, F) } \\
\text { Note: population overlaps } \\
\text { with Selevan } 1985\end{array}$ & $\begin{array}{l}169 \text { cases, } 469 \text { controls; } \\
\text { only } 53 \text { pregnancies } \\
\text { exposed }\end{array}$ & $\begin{array}{l}\text { Central Registry of Health } \\
\text { Care Personnel and a } \\
\text { questionnaire to head } \\
\text { hospital nurses regarding } \\
\text { each subjects' } \\
\text { occupational exposures } \\
\text { (type of occupational } \\
\text { exposure, shift work, etc.) } \\
\text { Note: Exposed nurses } \\
\text { were from non-oncology } \\
\text { departments (i.e., } \\
\text { anesthesia, intensive care, } \\
\text { operating room, or } \\
\text { internal medicine) where } \\
\text { exposure was relatively } \\
\text { uncommon }\end{array}$ & Medical records & $\begin{array}{l}\text { Logistic model of } \\
\text { conditional } \\
\text { likelihood function } \\
\text { adjusted for shift } \\
\text { work and co- } \\
\text { exposures (e.g., } \\
\text { ionizing radiation) }\end{array}$ & $\begin{array}{l}\text { No difference in odds of } \\
\text { spontaneous abortion } \\
\text { between cytostatic } \\
\text { agents } \geq \text { once weekly } \\
\text { and unexposed } \\
\text { (OR }=0.8,95 \% \\
\mathrm{CI}=0.3-1.7 ; 12 \\
\text { exposed cases versus } 41 \\
\text { unexposed cases) }\end{array}$ \\
\hline $\begin{array}{l}\text { Lawson et al. } \\
(2012)\end{array}$ & $\begin{array}{l}\text { Cohort, retrospective } \\
\text { (Nurses' Health Study II, } \\
\text { USA, 1993-2002, F) }\end{array}$ & $\begin{array}{l}775 \text { cases, } 6,707 \text { live } \\
\text { births; } 302 \text { exposed, } \\
\text { 7,180 unexposed }\end{array}$ & $\begin{array}{l}\text { Job title and self-reported } \\
\text { questionnaire, including } \\
\text { questions for each } \\
\text { trimester of pregnancy on } \\
\text { exposure to } 5 \text { occupational } \\
\text { exposures: anesthesia, } \\
\text { cancer chemotherapy } \\
\text { agents, anti-viral drugs, } \\
\text { sterilizing agents, and X- } \\
\text { ray radiation }\end{array}$ & $\begin{array}{l}\text { Self-reported } \\
\text { questionnaire on most } \\
\text { recent pregnancy; } \\
\text { defined as pregnancy } \\
\text { loss at }<20 \text { weeks of } \\
\text { gestation }\end{array}$ & $\begin{array}{l}\text { Logistic regression } \\
\text { adjusted for age; } \\
\text { multivariate } \\
\text { analysis included } \\
\text { all } 5 \text { work } \\
\text { exposures, parity, } \\
\text { shift work, and } \\
\text { hours worked }\end{array}$ & $\begin{array}{l}\text { Significantly higher } \\
\text { odds of spontaneous } \\
\text { abortion in nurses } \\
\text { exposed } \geq 1 \text { hour per } \\
\text { day compared to } \\
\text { unexposed pregnancies } \\
\text { (adjOR }=1.94,95 \% \\
\mathrm{CI}=1.32-2.86 ; 48 \\
\text { exposed cases); } \\
\text { appeared to be due to } \\
\text { spontaneous abortions } \\
\text { occurring at }<12 \text { weeks }\end{array}$ \\
\hline
\end{tabular}




\begin{tabular}{|c|c|c|c|c|c|c|}
\hline Study & $\begin{array}{c}\text { Study Design } \\
\text { (Occupation, Location, } \\
\text { Years Data Collected, } \\
\text { Sex) }\end{array}$ & $\begin{array}{c}\text { No. of Pregnancies } \\
\text { (Unless Otherwise } \\
\text { Stated) }\end{array}$ & Exposure Measure & $\begin{array}{c}\text { Outcome } \\
\text { Assessment }\end{array}$ & Analysis & Results \\
\hline $\begin{array}{l}\text { McDonald et al. } \\
\text { (1988b) }\end{array}$ & $\begin{array}{l}\text { Cohort, retrospective } \\
\text { (Working women, 1982- } \\
\text { 1984, Canada, F) }\end{array}$ & $\begin{array}{l}22,713 \text { (only } 63 \\
\text { pregnancies exposed to } \\
\text { cancer chemotherapy } \\
\text { agents) }\end{array}$ & $\begin{array}{l}\text { Job title and interviewer- } \\
\text { led questionnaire, and } \\
\text { chemical exposure } \\
\text { information from } \\
\text { published and unpublished } \\
\text { studies, and occupational } \\
\text { surveillance by local } \\
\text { health departments and } \\
\text { Montreal surveillance for } \\
\text { cancer, and other reports } \\
\text { Note: Medical } \\
\text { questionnaire also asked } \\
\text { nurses or physicians if } \\
\text { they administered cancer } \\
\text { chemotherapy agents in } \\
\text { the month after the first } \\
\text { missed menstrual period }\end{array}$ & $\begin{array}{l}\text { Interviewer-led } \\
\text { questionnaire on } \\
\text { previous pregnancies; } \\
\text { defined as pregnancy } \\
\text { at }<28 \text { weeks of } \\
\text { gestation }\end{array}$ & $\begin{array}{l}\text { Poisson regression; } \\
\text { evaluated factors: } \\
\text { age, gravidity, } \\
\text { history of previous } \\
\text { abortion, ethnic } \\
\text { group, educational } \\
\text { level as well as } \\
\text { smoking habit and } \\
\text { alcohol } \\
\text { consumption }\end{array}$ & $\begin{array}{l}\text { No association with } \\
\text { spontaneous abortion } \\
\text { compared to unexposed } \\
\text { pregnancies } \\
\text { (O/E = 0.97, } 13 \text { exposed } \\
\text { cases); maternal age, } \\
\text { gravidity, previous } \\
\text { spontaneous abortion, } \\
\text { smoking, and alcohol } \\
\text { consumption were } \\
\text { significant factors [but } \\
\text { the data do not appear } \\
\text { to be adjusted for these } \\
\text { significant factors] }\end{array}$ \\
\hline Medková (1991a) & $\begin{array}{l}\text { Cross-sectional } \\
\text { (Physicians, nurses, auxiliary } \\
\text { workers, Czech Republic, } \\
\text { Year NR, FM) }\end{array}$ & $\begin{array}{l}74 \text { exposed, } \\
11 \text { unexposed (to } \\
\text { partners of exposed } \\
\text { subjects) }\end{array}$ & $\begin{array}{l}\text { Job title and interviewer- } \\
\text { led questionnaire }(\geq 5 \\
\text { years, } \mathrm{n}=25 ; 6-10 \text { years, } \\
\mathrm{n}=14 ; 11-15 \text { years, } \mathrm{n}=4 \text {; } \\
\text { and } 16-20 \text { years, } \mathrm{n}=1) \\
\text { Note: All exposed } \\
\text { personnel used gloves and } \\
\text { gowns; isolated room for } \\
\text { drug preparation only } \\
\text { used by 25\% of exposed } \\
\text { subjects }\end{array}$ & $\begin{array}{l}\text { Interviewer-led } \\
\text { questionnaire; [no } \\
\text { definition provided for } \\
\text { early and late } \\
\text { spontaneous abortion] }\end{array}$ & NR & $\begin{array}{l}\text { Higher number of early } \\
\text { spontaneous abortions } \\
\text { compared to unexposed } \\
\text { pregnancies (early: } 8 \\
\text { exposed vs. } 0 \\
\text { unexposed); whereas, } \\
\text { there were } 2 \text { late } \\
\text { spontaneous abortions } \\
\text { in unexposed versus } 0 \\
\text { in exposed }\end{array}$ \\
\hline
\end{tabular}




\begin{tabular}{|c|c|c|c|c|c|c|}
\hline Study & $\begin{array}{c}\text { Study Design } \\
\text { (Occupation, Location, } \\
\text { Years Data Collected, } \\
\text { Sex) }\end{array}$ & $\begin{array}{c}\text { No. of Pregnancies } \\
\text { (Unless Otherwise } \\
\text { Stated) }\end{array}$ & Exposure Measure & $\begin{array}{l}\text { Outcome } \\
\text { Assessment }\end{array}$ & Analysis & Results \\
\hline $\begin{array}{l}\text { Peelen et al. } \\
\text { (1999) }\end{array}$ & $\begin{array}{l}\text { Cross-sectional (Nurses, the } \\
\text { Netherlands, 1990-1997, F) } \\
\text { Note: Same data as } \\
\text { Fransman et al. } 2007 \text { c }\end{array}$ & $\begin{array}{l}249 \text { exposed, } 1,010 \\
\text { unexposed }\end{array}$ & $\begin{array}{l}\text { Job title, self-reported } \\
\text { questionnaire, } \\
\text { biomonitoring, and } \\
\text { environmental monitoring }\end{array}$ & $\begin{array}{l}\text { Self-reported } \\
\text { questionnaire on most } \\
\text { recent pregnancies; } \\
\text { [definition same as } \\
\text { Fransman et al. 2007c] }\end{array}$ & $\begin{array}{l}\text { [Logistic } \\
\text { regression]; } \\
\text { adjusted for age, } \\
\text { coffee } \\
\text { consumption, } \\
\text { smoking, alcohol } \\
\text { consumption, } \\
\text { pregnancy, } \\
\text { physical strain, and } \\
\text { co-exposures (e.g., } \\
\text { ionizing radiation, } \\
\text { disinfectants), as } \\
\text { needed }\end{array}$ & $\begin{array}{l}\text { Non-significant, but } \\
\text { slightly higher, odds of } \\
\text { spontaneous abortion } \\
\text { compared to unexposed } \\
\text { pregnancies } \\
\text { (adjOR = 1.4, 95\% } \\
\text { CI = 0.8-2.6; } 18 \\
\text { exposed cases); 30\% of } \\
\text { urine samples from } \\
\text { exposed nurses tested } \\
\text { positive for CP and 40\% } \\
\text { tested positive for IF; } \\
\text { unexposed subjects } \\
\text { were not tested }\end{array}$ \\
\hline $\begin{array}{l}\text { Schaumburg and } \\
\text { Olsen (1990b) }\end{array}$ & $\begin{array}{l}\text { Cohort, retrospective } \\
\text { (Pharmacy assistants, } \\
\text { Denmark, 1979-1984, F) }\end{array}$ & $\begin{array}{l}\text { 1,882 births, } 177 \\
\text { spontaneous abortions, } \\
109 \text { induced abortions; } \\
\text { only } 8 \text { pregnancies } \\
\text { exposed to cancer } \\
\text { chemotherapy agents }\end{array}$ & $\begin{array}{l}\text { Membership in national } \\
\text { union of pharmacy } \\
\text { assistants and self-reported } \\
\text { questionnaire }\end{array}$ & Medical records & $\begin{array}{l}\text { Unconditional } \\
\text { logistic regression } \\
\text { model }\end{array}$ & $\begin{array}{l}\text { No spontaneous } \\
\text { abortions were reported } \\
\text { for the } 8 \text { pregnancies of } \\
\text { occupationally exposed } \\
\text { personnel }\end{array}$ \\
\hline $\begin{array}{l}\text { Selevan et al. } \\
(1985)\end{array}$ & $\begin{array}{l}\text { Case-control (Nurses, } \\
\text { Finland, 1973-1980, F) }\end{array}$ & $\begin{array}{l}124 \text { cases, } 321 \text { controls; } \\
\text { only } 92 \text { pregnancies } \\
\text { exposed }\end{array}$ & $\begin{array}{l}\text { Central Registry of Health } \\
\text { Care Personnel and self- } \\
\text { reported questionnaire } \\
\text { Note: Exposed nurses } \\
\text { identified as having high } \\
\text { usage of cancer } \\
\text { chemotherapy agents }\end{array}$ & Medical records & $\begin{array}{l}\text { Conditional } \\
\text { logistic regression; } \\
\text { factors considered: } \\
\text { smoking, alcohol } \\
\text { use, medications in } \\
\text { first trimester, } \\
\text { gravidity, prior } \\
\text { fetal loss, exposure } \\
\text { prior to pregnancy }\end{array}$ & $\begin{array}{l}\text { Significantly higher } \\
\text { odds of spontaneous } \\
\text { abortion with first } \\
\text { trimester exposure } \\
\text { (OR = 2.3, 95\% } \\
\text { CI = 1.2-4.39; } 18 \\
\text { exposed cases); prior } \\
\text { fetal loss was } \\
\text { significant factor and } \\
\text { results were the same } \\
\text { when adjusted for this } \\
\text { factor; exposure prior to } \\
\text { pregnancy NS }\end{array}$ \\
\hline
\end{tabular}




\begin{tabular}{|c|c|c|c|c|c|c|}
\hline Study & $\begin{array}{c}\text { Study Design } \\
\text { (Occupation, Location, } \\
\text { Years Data Collected, } \\
\text { Sex) }\end{array}$ & $\begin{array}{c}\text { No. of Pregnancies } \\
\text { (Unless Otherwise } \\
\text { Stated) }\end{array}$ & Exposure Measure & $\begin{array}{c}\text { Outcome } \\
\text { Assessment }\end{array}$ & Analysis & Results \\
\hline Skov et al. (1992) & $\begin{array}{l}\text { Cohort, retrospective } \\
\text { (Nurses, Denmark, 1977- } \\
\text { 1988, F) }\end{array}$ & $\begin{array}{l}281 \text { exposed, } 809 \\
\text { unexposed }\end{array}$ & $\begin{array}{l}\text { Employment records of } 5 \\
\text { oncology hospitals, and } \\
\text { data from head nurses } \\
\text { regarding drug handling } \\
\text { and administration of } \\
\text { individual subjects; } \\
\text { evaluated data as exposed } \\
\text { versus unexposed and by } \\
\text { level of exposure [details } \\
\text { not provided] versus } \\
\text { unexposed }\end{array}$ & Medical records & $\begin{array}{l}\text { Logistic regression } \\
\text { analysis with } \\
\text { adjustment for } \\
\text { maternal age }\end{array}$ & $\begin{array}{l}\text { No difference in odds of } \\
\text { spontaneous abortion } \\
\text { compared to unexposed } \\
\text { nurses (adjOR = 0.74, } \\
95 \% \mathrm{CI}=0.40-1.38 ; 18 \\
\text { exposed cases); } \\
\text { spontaneous abortion } \\
\text { rate was similar when } \\
\text { considering first } \\
\text { pregnancies only; no } \\
\text { exposure-response } \\
\text { observed }\end{array}$ \\
\hline $\begin{array}{l}\text { Stücker et al. } \\
\text { (1990) }\end{array}$ & $\begin{array}{l}\text { Cross-sectional (Nurses, } \\
\text { France, 1985-1986, F) }\end{array}$ & $\begin{array}{l}139 \text { exposed, } 357 \\
\text { unexposed }\end{array}$ & $\begin{array}{l}\text { Job title and interviewer- } \\
\text { led questionnaire } \\
\text { Note: At time of study, no } \\
\text { special protective } \\
\text { equipment was in } \\
\text { operation and use of PPE } \\
\text { was not uniform }\end{array}$ & $\begin{array}{l}\text { Interviewer-led } \\
\text { questionnaire; defined } \\
\text { as pregnancy loss at }<28 \\
\text { weeks of gestation }\end{array}$ & $\begin{array}{l}\text { Logistic regression } \\
\text { analysis; evaluated } \\
\text { effect of maternal } \\
\text { age, parity and } \\
\text { smoking, exposure } \\
\text { duration and } \\
\text { frequency of } \\
\text { handling }\end{array}$ & $\begin{array}{l}\text { Significantly higher } \\
\text { odds of spontaneous } \\
\text { abortion in the first } \\
\text { exposed pregnancy } \\
\text { compared to unexposed } \\
\text { pregnancies } \\
\text { (adjOR = 1.7, 95\% } \\
\mathrm{CI}=1.0-2.8 \text { ); } 36 \\
\text { exposed cases; } \\
\text { exclusion of } 24 \text { cases } \\
\text { prior to pregnancy did } \\
\text { not change significance } \\
\text { (rate ratio = 1.7, } 95 \% \\
\mathrm{CI}=1.1-2.5 \text { ) }\end{array}$ \\
\hline
\end{tabular}




\begin{tabular}{|c|c|c|c|c|c|c|}
\hline Study & $\begin{array}{c}\text { Study Design } \\
\text { (Occupation, Location, } \\
\text { Years Data Collected, } \\
\text { Sex) }\end{array}$ & $\begin{array}{c}\text { No. of Pregnancies } \\
\text { (Unless Otherwise } \\
\text { Stated) }\end{array}$ & Exposure Measure & $\begin{array}{c}\text { Outcome } \\
\text { Assessment }\end{array}$ & Analysis & Results \\
\hline $\begin{array}{l}\text { Taskinen et al. } \\
\text { (1986) }\end{array}$ & $\begin{array}{l}\text { Case-control } \\
\text { (Pharmaceutical industry } \\
\text { workers, Finland, 1973- } \\
\text { 1980, F) }\end{array}$ & $\begin{array}{l}44 \text { cases, } 130 \text { controls; } \\
\text { only } 6 \text { exposed } \\
\text { pregnancies }\end{array}$ & $\begin{array}{l}\text { Factory physician } \\
\text { completed-questionnaire } \\
\text { on exposure during } \\
\text { pregnancy through health } \\
\text { cards, labor protection } \\
\text { chiefs, and department } \\
\text { foremen regarding } \\
\text { occupation, main tasks, } \\
\text { exposures, lifting, } \\
\text { standing, or sitting; } \\
\text { employed at least one } \\
\text { week during the first } \\
\text { trimester }\end{array}$ & Medical records & $\begin{array}{l}\text { Logistic regression } \\
\text { analysis based on } \\
\text { conditional } \\
\text { maximum } \\
\text { likelihood }\end{array}$ & $\begin{array}{l}\text { No statistically } \\
\text { significant higher odds } \\
\text { of spontaneous } \\
\text { abortions compared to } \\
\text { unexposed pregnancies } \\
(\mathrm{OR}=2.8,95 \% \\
\mathrm{CI}=0.6-1.4 ; 3 \text { exposed } \\
\text { cases })\end{array}$ \\
\hline $\begin{array}{l}\text { Taskinen et al. } \\
\text { (1994) }\end{array}$ & $\begin{array}{l}\text { Case-control (Laboratory } \\
\text { assistants, Finland, 1970- } \\
\text { 1986, F) }\end{array}$ & $\begin{array}{l}206 \text { cases, } 329 \text { controls; } \\
\text { only } 8 \text { exposed } \\
\text { pregnancies }\end{array}$ & $\begin{array}{l}\text { Employment records and } \\
\text { self-reported questionnaire } \\
\text { regarding frequency of } \\
\text { specific exposures during } \\
\text { first trimester }\end{array}$ & Medical records & $\begin{array}{l}\text { Logistic regression } \\
\text { based on } \\
\text { conditional } \\
\text { likelihood; } \\
\text { covariate included } \\
\text { smoking, alcohol } \\
\text { use, employment } \\
\text { status }\end{array}$ & $\begin{array}{l}\text { No statistically } \\
\text { significant higher odds } \\
\text { of spontaneous abortion } \\
\text { compared to unexposed } \\
\text { pregnancies } \\
\text { (adjOR = 4.0, } 95 \% \\
\text { CI = 0.8-19.3; } 5 \\
\text { exposed cases) }\end{array}$ \\
\hline
\end{tabular}




\begin{tabular}{|c|c|c|c|c|c|c|}
\hline Study & $\begin{array}{c}\text { Study Design } \\
\text { (Occupation, Location, } \\
\text { Years Data Collected, } \\
\text { Sex) }\end{array}$ & $\begin{array}{c}\text { No. of Pregnancies } \\
\text { (Unless Otherwise } \\
\text { Stated) }\end{array}$ & Exposure Measure & $\begin{array}{c}\text { Outcome } \\
\text { Assessment }\end{array}$ & Analysis & Results \\
\hline $\begin{array}{l}\text { Valanis et al. } \\
\text { (1999) }\end{array}$ & $\begin{array}{l}\text { Cross-sectional (Nurses, } \\
\text { nurses' aides, pharmacists, } \\
\text { and pharmacy technicians, } \\
\text { USA, 1988-1989, FM) }\end{array}$ & $\begin{array}{l}\text { 1,448 exposed, 5,297 } \\
\text { unexposed }\end{array}$ & $\begin{array}{l}\text { Job title and self-reported } \\
\text { questionnaire } \\
\text { Note: Exposed subjects } \\
\text { were involved in mixing, } \\
\text { administering, and/or } \\
\text { handling the excreta of } \\
\text { patients administered } \\
\text { cancer chemotherapy } \\
\text { agents }\end{array}$ & $\begin{array}{l}\text { Self-reported } \\
\text { questionnaire; defined } \\
\text { as pregnancy loss at } \leq 20 \\
\text { weeks }\end{array}$ & $\begin{array}{l}\text { Logistic regression } \\
\text { for (1) } \\
\text { independence } \\
\text { model and (2) } \\
\text { generalized } \\
\text { estimating } \\
\text { equations model; } \\
\text { all models adjusted } \\
\text { for age, gravidity } \\
\text { at time of } \\
\text { pregnancy, } \\
\text { maternal smoking } \\
\text { during pregnancy; } \\
\text { outcome of prior } \\
\text { fetal loss }\end{array}$ & $\begin{array}{l}\text { Significantly higher } \\
\text { odds of spontaneous } \\
\text { abortion in pregnancies } \\
\text { of exposed female } \\
\text { subjects exposed during } \\
\text { pregnancy } \\
\text { (adjOR = 1.5, 95\% } \\
\mathrm{CI}=1.2-1.8 ; 223 \\
\text { exposed cases) or prior } \\
\text { to pregnancy } \\
\text { (adjOR = 1.5, 95\% } \\
\mathrm{CI}=1.3-1.9) \text { compared } \\
\text { to unexposed } \\
\text { pregnancies; } \\
\text { pregnancies of exposed } \\
\text { female subjects affected } \\
\text { more than pregnancies } \\
\text { of spouses of exposed } \\
\text { male subjects }\end{array}$ \\
\hline $\begin{array}{l}\text { Walusiak et al. } \\
\text { (2003) }\end{array}$ & $\begin{array}{l}\text { Cross-sectional (Physicians, } \\
\text { nurses, orderlies, Year NR, } \\
\text { Poland, FM) }\end{array}$ & $\begin{array}{l}84 \text { exposed, } 169 \\
\text { unexposed }\end{array}$ & $\begin{array}{l}\text { Job title and self-reported } \\
\text { questionnaire } \\
\text { (11.0 } \pm 9.3 \text { years, range } 5- \\
44 \text { years) }\end{array}$ & $\begin{array}{l}\text { [Interviewer-led] } \\
\text { questionnaire; defined } \\
\text { as pregnancy loss at }<24 \\
\text { weeks of gestation }\end{array}$ & $\begin{array}{l}\text { Mann-Whitney U- } \\
\text { test }\end{array}$ & $\begin{array}{l}\text { Greater, but NS, } \\
\text { number of spontaneous } \\
\text { abortions compared to } \\
\text { unexposed nurses } \\
\text { (unexposed = } 9 \text { cases, } \\
5.3 \% \text { prevalence; } \\
\text { exposed = } 7 \text { cases, } 8.3 \% \\
\text { prevalence) }\end{array}$ \\
\hline
\end{tabular}




\begin{tabular}{|c|c|c|c|c|c|c|}
\hline Study & $\begin{array}{c}\text { Study Design } \\
\text { (Occupation, Location, } \\
\text { Years Data Collected, } \\
\text { Sex) }\end{array}$ & $\begin{array}{c}\text { No. of Pregnancies } \\
\text { (Unless Otherwise } \\
\text { Stated) }\end{array}$ & Exposure Measure & $\begin{array}{c}\text { Outcome } \\
\text { Assessment }\end{array}$ & Analysis & Results \\
\hline $\begin{array}{l}\text { Zhang et al. } \\
\text { (2016) }\end{array}$ & $\begin{array}{l}\text { Cross-sectional (Nurses, } \\
\text { China, } 2011 \text { pre-PIVAS and } \\
2013 \text { (post-PIVAS) in } \\
\text { Hospital A, F) }\end{array}$ & $\begin{array}{l}\text { Pre-PIVAS: } 106 \\
\text { unexposed, } 30 \text { exposed } \\
\text { non-oncology, } 82 \\
\text { exposed oncology; Post- } \\
\text { PIVAS: } 108 \text { unexposed, } \\
29 \text { exposed non- } \\
\text { oncology, } 87 \text { exposed } \\
\text { oncology, and } 112 \\
\text { exposed oncology } \\
\text { nurses at Hospital B) }\end{array}$ & $\begin{array}{l}\text { Job title and self-reported } \\
\text { questionnaire ( } \geq 2 \text { years in } \\
\text { current job) } \\
\text { Note: All exposed nurses } \\
\text { involved in mixing and/or } \\
\text { administration of cancer } \\
\text { chemotherapy agents }\end{array}$ & $\begin{array}{l}\text { Self-reported } \\
\text { questionnaire; } \\
\text { pregnancy loss at }<24 \\
\text { weeks of gestation }\end{array}$ & $\begin{array}{l}\text { [Student] t-test, } \\
\text { Chi-square, and } \\
\text { Wilcoxon [signed- } \\
\text { rank sum] test }\end{array}$ & $\begin{array}{l}\text { No difference in rate of } \\
\text { spontaneous abortion } \\
\text { compared to unexposed } \\
\text { nurses prior to PIVAS } \\
\text { at Hospital A; after } \\
\text { implementation of } \\
\text { PIVAS, the rate of } \\
\text { spontaneous abortions } \\
\text { in exposed oncology } \\
\text { nurses was significantly } \\
\text { (p<0.05) lower } \\
\text { compared to pre-PIVAS } \\
\text { values as well as non- } \\
\text { significant reductions in } \\
\text { the rates for unexposed } \\
\text { and exposed non- } \\
\text { oncology nurses. } \\
\text { Significantly (p<0.01) } \\
\text { higher rate of } \\
\text { spontaneous abortions } \\
\text { was reported for } \\
\text { exposed oncology } \\
\text { nurses at Hospital B (no } \\
\text { PIVAS) compared to } \\
\text { post-PIVAS exposed } \\
\text { oncology nurses at } \\
\text { Hospital A }\end{array}$ \\
\hline
\end{tabular}

adjOR = adjusted odds ratio; $\mathrm{CI}=$ confidence interval, $\mathrm{CP}=$ cyclophosphamide, $\mathrm{F}=$ female, $\mathrm{IF}=$ ifosfamide; $\mathrm{M}=$ male, $\mathrm{NR}=$ not reported, $\mathrm{NS}=$ not significant, $\mathrm{OR}=$ odds ratio, PIVAS = pharmacy intravenous admixture service, and USA = United States of America, wk = week. 
The remaining nine studies evaluating spontaneous abortion were less informative for a variety of reasons. Hemminki et al. (1985) conducted a case-control study of the same population of nurses in France as Selevan et al. (1985) to evaluate the association with occupational exposure to anesthesia gases, sterilizing agents, ionizing radiation, cancer chemotherapy agents, and other exposures. The authors observed no association between occupational exposure to cancer chemotherapy agents and spontaneous abortion (Hemminki et al. 1985); however, the authors stated that the nurses were from hospital departments with reduced exposure to cancer chemotherapy agents (i.e., anesthesia surgery, intensive care, operating room or internal medicine department of a general hospital) relative to the nurses with high levels of handling and administering these agents reported in the study by Selevan et al. (1985). A retrospective study of oncology nurses in five hospitals in Denmark from 1977-1988 reported that spontaneous abortion was not associated with occupational exposure to cancer chemotherapy agents when evaluating exposed versus unexposed nurses or in an exposure level (low, moderate, high exposure) analysis conducted by head nurses consulting hospital drug-handling logs (Skov et al. 1992). However, the authors did not provide details on how they categorized the nurses into the exposure levels, and there was no information on use of personal protective equipment; the authors hypothesized that part of the lack of effect might be due to the implementation of safety handling procedures for cancer chemotherapy agents after 1980.

One retrospective cohort study of 2,168 pregnancies of pharmacy assistants in Denmark observed no spontaneous abortions among the eight pregnancies exposed to cancer chemotherapy agents; however, the workers may not have been highly exposed (characterized as daily or weekly exposure versus unexposed) (Schaumburg and Olsen 1990b). No association of spontaneous abortion with occupational exposure to cancer chemotherapy agents was observed in a cross-sectional study assessing occupational exposures of nurses and doctors in Canada; however, there was no information provided about the tasks or frequency of drug handling of the exposed individuals (McDonald et al. 1988b). Two case-control studies evaluating the influence of various occupational exposures of pharmaceutical workers (Taskinen et al. 1986) and laboratory assistants (Taskinen et al. 1994) reported higher, but non-significant, odds for spontaneous abortion with exposure to cancer chemotherapy agents; however, these studies included very few exposed pregnancies (6-8 pregnancies) with very few cases (3-5 cases) and the range of exposure during pregnancy was insufficient to determine a difference between groups (controls: 1-2 days per week versus exposed: $\geq 3$ days per week). A cross-sectional study of physicians, nurses, and auxiliary workers handling cancer chemotherapy agents in the Czech Republic reported a higher occurrence of early spontaneous abortions in exposed subjects compared to unexposed spouses of the exposed subjects across all pregnancies (Medková 1991a); however, the authors did not provide a definition of early spontaneous abortion, no statistical analyses were reported, and there were only two unexposed subjects with pregnancy outcome data compared to 38 exposed subjects with pregnancy outcome data. A cross-sectional study of doctors, nurses, and orderlies in Poland reported that there was no difference in spontaneous abortion in the exposed subjects versus the unexposed personnel (Walusiak et al. 2003). The authors reported that doctors were the least exposed of the medical personnel, but they do not explain how they characterized exposure and they did not run an analysis with only the nurses and orderlies (Walusiak et al. 2003). Finally, a cross-sectional study of oncology nurses in Egypt employed in the same position for $>10$ years reported a significantly $(\mathrm{p}<0.05)$ higher rate of abortions - which was not defined, but was presumed to be spontaneous abortions - relative to controls (22.6\%) compared to unexposed nurses (10.3\%); the authors 
reported poor adherence to safety measures of the exposed nurses, such as eating in the drug administration area (45.7\%) and only $42.9 \%$ used gloves when cleaning up spills or handling patient waste.

\section{Risk-of-bias Assessment}

Risk-of-bias assessments were conducted on all studies included in the evidence synthesis for spontaneous abortion (Figure D-2 and Figure D-3). Approximately 60\% of the studies reporting on the occurrence of spontaneous abortion were rated probably low risk of bias for confounding (e.g., appropriate confounders were considered and adjusted when necessary), exposure characterization, and outcome assessment.

Most studies (10 of 16) were rated probably low or definitely low risk of bias for confounding. Schaumburg and Olsen (1990b) did not adjust for confounders or control for multiple exposures among the pharmacy assistants working with cancer chemotherapy drugs on a daily or weekly basis; however, this study was rated as probably low risk of bias because no spontaneous abortions were observed among the eight exposed pregnancies. One study was rated as probably high risk of bias (Medková 1991a); the author did not adjust for differences in age or smoking, and it was not clear whether both the male employees and their pregnant spouses were smokers or whether second hand smoke was addressed. Five studies were rated probably high risk of bias for lack of adjustment for confounding variables (Elshamy et al. 2010; McDonald et al. 1988b; Skov et al. 1992; Taskinen et al. 1986; Walusiak et al. 2003). One study of female pharmaceutical workers did not appear to adjust for possible co-exposures (e.g., ionizing radiation) in their analysis, and the authors did not report whether they evaluated for confounders of smoking or alcohol consumption (Taskinen et al. 1986). McDonald et al. (1988b) identified significant differences in the prevalence of several confounders (i.e., maternal age, gravidity, previous spontaneous abortion, smoking, and alcohol consumption) between the exposed and unexposed nurses and doctors, but did not report whether they adjusted for these confounders. Skov et al. (1992) did not evaluate smoking or alcohol consumption in their retrospective cohort of oncology nurses; however, the authors comment that nurses were likely to have similar rates of these two possible confounders, regardless of occupational exposure. A study of oncology nurses in Egypt did not report an assessment of potential confounders for either exposed or controls (e.g., smoking, ionizing radiation) (Elshamy et al. 2010). Finally, Walusiak et al. (2003) reported data on the sex, age, and smoking status of the exposed and unexposed nurses in their study, but did not present any statistical analysis evaluating possible differences in these confounders.

Ten of 16 studies were rated probably low risk of bias for exposure characterization. For example, Fransman et al. (2007c) estimated exposure using data on self-reported tasks of oncology nursing staff (preparation, administration, handling patient urine, washing a patient, removing bed sheets, cleaning toilets) and PPE use with task-based exposure measurements of glove and hand (dermal) contamination of cyclophosphamide. In another study, Lawson et al. (2012) characterized exposure from the Nurses' Health Study, a well-established cohort, using a self-reported questionnaire with questions regarding occupational exposure to cancer chemotherapy agents as well as other genetic toxicant exposures (e.g., anesthetic gases, sterilizing agents, x-rays), shiftwork, and anti-viral medication. The questionnaire included examples for each class of exposures, which has been demonstrated to improve the accuracy of the exposure recall (Teschke et al. 2002). While use of questionnaires (self-report or interviewer- 
led) to characterize exposure in retrospective cohort studies can be influenced by recall bias, support for accurate recall of exposure by medical staff has been demonstrated by a study of pharmacists whose self-reported questionnaire was corroborated by their supervisor (McDiarmid et al. 1992). Six studies were rated probably high risk of bias for exposure characterization (Hemminki et al. 1985; McDonald et al. 1988b; Skov et al. 1992; Taskinen et al. 1994; Taskinen et al. 1986; Walusiak et al. 2003). Two studies were rated probably high risk of bias for a lack of details regarding exposure characterization; for example, Walusiak et al. (2003) only provided job title as the description of physicians, nurses, and orderlies handling cancer chemotherapy agents although they stated that doctors were less exposed, and Skov et al. (1992) provided no description of the semi-quantitative categories of exposure of oncology nurses from two hospitals (Skov et al. 1992). Two studies did not provide a sufficient range or variance in exposure measures across groups (Taskinen et al. 1994; Taskinen et al. 1986); e.g., these studies compared female laboratory workers (Taskinen et al. 1994) or pharmaceutical factory workers (Taskinen et al. 1986) exposed (1994) 1-2 days per week (considered rarely handled) versus $\geq 3$ days per week (considered frequently handled) during pregnancy. Another study reported the exposure of each participant was assessed by questionnaire of the head nurse of the hospital on behalf of the study participants, which may lead to high potential for recall bias (Hemminki et al. 1985). Finally, McDonald et al. (1988b) exposure was assessed by interviewerled questionnaire to doctors and nurses regarding what chemicals (including antineoplastic agents) they were exposed to while at work in the first four weeks past their first missed menstrual period; thus, the study assessed only whether they were exposed or not exposed to cancer chemotherapy agents with no details about frequency of use or personal protective equipment use.

Regarding outcome assessment, most studies included in the evidence synthesis were rated definitely low risk of bias or probably low risk of bias because they assessed outcome by identifying cases from hospital discharge records or collected data from questionnaires from which the participants were not aware of the connection between outcome and exposure. For example, Fransman et al. (2007c) and Peelen et al. (1999) conducted their self-reported questionnaire of oncology nurses in the Netherlands as a study of reproductive outcomes in nurses. The interviewer-led questionnaire by Stucker et al. (1990) presented the purpose of the study as an assessment of reproductive characteristics in hospital personnel. Similarly, exposure was not likely to influence the outcome assessment from the Nurses' Health Study II as it is a well-established cohort study, which collects data on all types of lifestyle, exposure and health data (Lawson et al. 2012). While recall error can be a limitation of questionnaires assessing health outcomes, women have accurate recall of pregnancy outcomes (Axelsson 1990; Joffe et al. 1993), and nurses have accurate recall of health data (Colditz et al. 1986; Colditz et al. 1987). Thus, even when participants were advised of the purpose of the study as in three studies reporting on spontaneous abortion (McDonald et al. 1988b; Valanis et al. 1999; Walusiak et al. 2003), it would not likely influence their recall of spontaneous abortion or other pregnancy outcomes. Two studies were rated as probably high risk of bias due to a lack of definitions for early and late spontaneous abortions (Medková 1991a) or abortions [presumed to be spontaneous abortions] (Elshamy et al. 2010).

\section{Confidence Ratings and Level-of-evidence Conclusions}

The initial rating of moderate confidence in the body of evidence for spontaneous abortion was not downgraded or upgraded for any factor (Figure 5). The confidence rating was based on seven 
studies with the lowest risk of bias (defined as none or no more than one probably high risk-ofbias rating for one of three key questions of confounding, exposure characterization, and outcome assessment) and the most highly exposed personnel (Fransman et al. 2007c; Lawson et al. 2012; Peelen et al. 1999; Selevan et al. 1985; Stücker et al. 1990; Valanis et al. 1999; Zhang et al. 2016). The remaining studies had higher risk of bias and/or provided less detail to determine level of drug handling; thus, there were no changes in the initial rating of moderate confidence in the body of evidence after considering factors that may increase or decrease confidence (Figure 5). Thus, the final rating was moderate confidence in the body of evidence. The final rating of moderate confidence directly translates into a moderate level of evidence that occupational exposure to cancer chemotherapy agents is associated with spontaneous abortion.

\begin{tabular}{|c|c|c|c|c|c|c|c|c|c|c|c|}
\hline \multirow[b]{2}{*}{$\begin{array}{l}\text { Initial Confidence for } \\
\text { Each Body of Evidence } \\
\text { (\# of Studies) }\end{array}$} & \multicolumn{5}{|c|}{$\begin{array}{l}\text { Factors Decreasing Confidence } \\
\text { (““” if no concern; “ “”” if serious } \\
\text { concern to downgrade confidence) }\end{array}$} & \multicolumn{5}{|c|}{$\begin{array}{c}\text { Factors Increasing Confidence } \\
\text { (“-” if not present; “ } \uparrow ” \text { if sufficient } \\
\text { to upgrade confidence) }\end{array}$} & \multirow[b]{2}{*}{$\begin{array}{l}\text { Final } \\
\text { Confidence } \\
\text { Rating }\end{array}$} \\
\hline & 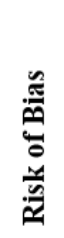 & 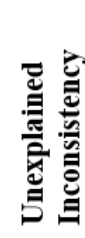 & 岂 & 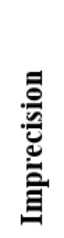 & 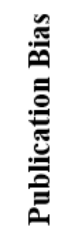 & 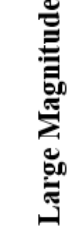 & 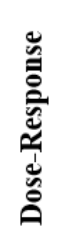 & 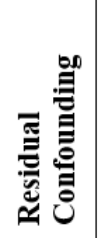 & 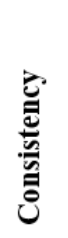 & $\dot{\bar{g}}$ & \\
\hline \multicolumn{12}{|l|}{ Spontaneous Abortion $\mathrm{p}^{\mathrm{a}, \mathrm{b}}$} \\
\hline $\begin{array}{l}\text { Initial Moderate } \\
\text { ( } 4 \text { cohort [retrospective], } \\
4 \text { case-controlc) }\end{array}$ & - & - & - & - & - & - & - & - & - & - & Moderate \\
\hline $\begin{array}{l}\text { Initial Low } \\
(6 \text { cross-sectionald })\end{array}$ & - & - & - & - & - & - & - & - & - & - & Low \\
\hline
\end{tabular}

Figure 5. Confidence in the Body of Evidence for Spontaneous Abortions

aSpontaneous abortion references: (Fransman et al. 2007c; Hemminki et al. 1985; Lawson et al. 2012; Medková 1991a; Peelen et al. 1999; Schaumburg and Olsen 1990b; Selevan et al. 1985; Skov et al. 1992; Stücker et al. 1990; Taskinen et al. 1994; Taskinen et al. 1986; Valanis et al. 1999; Walusiak et al. 2003; Zhang et al. 2016).

${ }^{b}$ The final confidence in the body of literature was based on seven high-quality studies of highly exposed workers that reported higher case numbers of spontaneous abortions, including: one retrospective cohort (Lawson et al. 2012), one case-control (Selevan et al. 1985), and five cross-sectional studies (Fransman et al. 2007c; Lawson et al. 2012; Peelen et al. 1999; Selevan et al. 1985; Stücker et al. 1990; Valanis et al. 1999; Zhang et al. 2016).

'One research group conducted case-control studies of the same population of nurses in France; however, the nurses evaluated in each study were slightly different: Selevan et al. (1985) evaluated nurses with high usage of cancer chemotherapy agents and Hemminki et al. (1985) studied the occupational exposure in nurses from departments of the hospitals with lesser exposure to these agents.

${ }^{\mathrm{d}}$ Two cross-sectional studies evaluated the same study population and data set using different exposure characterization methods: job title and work tasks only (Peelen et al. 1999) or estimated dermal exposure based on job title, work tasks and measured glove and skin contamination (Fransman et al. 2007c).

\section{Genetic Toxicity: DNA Damage}

Genetic toxicity assays have been used to evaluate whether occupational exposure to cancer chemotherapy agents results in chromosomal or DNA damage. These types of assays are often used in surveillance programs as biomarkers of exposure (i.e., to determine if occupational exposure to the chemical(s) of interest has occurred) (Kirsch-Volders et al. 2011; Mateuca et al. 2006). Nearly all of the classes of cancer chemotherapy agents are known to induce genetic toxicity [reviewed in Suspiro and Prista (2011) and Alam et al. (2011)]. NTP evaluated the 
effects of occupational exposure to cancer chemotherapy agents on three types of genetic toxicity assays in this review: induction of structural CA, MN, and DNA damage measured by the alkaline comet assay. All three of these assays have standardized testing protocols validated by the OECD, and are used to evaluate genetic toxicity in both epidemiological studies and laboratory animal toxicity testing of environmental and occupational exposures (OECD 2016a; 2016b; 2016c). Both CA and MN induction are considered early biomarkers of effect of cancer incidence (Bonassi et al. 2007; Bonassi et al. 2004; Norppa et al. 2006) and are frequently included in support of a carcinogenic agent listing in IARC and NTP's Report on Carcinogens (IARC 1966; 1980; 1981; 1990; 2000; NTP 2016). There is much interest in the comet assay to measure DNA damage as a biomarker of effect in environmental and occupational biomonitoring due to its affordability and relative ease of use (Valverde and Rojas 2009; Zare Sakhvidi et al. 2016); however, its association to cancer has not been established. All three assays rely on cells obtained in samples of peripheral blood.

The NTP evaluation identified 66 studies of 53 unique populations reporting structural CA, MN induction, and/or DNA damage measured by the comet assay (Table F-1). The available epidemiological studies in the body of evidence that evaluated the association between occupational exposure to cancer chemotherapy and the three DNA damage assays included: one prospective cohort study of pharmacists (Roth et al. 1994), one longitudinal cohort study of nurses (Mader et al. 2009), and 64 cross-sectional studies of medical personnel (e.g., nurses, physicians, pharmacists, pharmacy technicians) as well as chemists, laboratory assistants, and production or manufacturing workers). Most of the studies evaluated nursing or pharmacy personnel, while drug production, manufacturing employees, chemists, and laboratory assistants were the least studied (only four studies). The studies varied greatly in size (from 12 to 594 subjects), and length of time in job position (1 month to 30 years). The studies originated from 27 different countries, mainly in Europe $(n=42)$; there were three studies from the United States (all on the same study population) and one study from Canada.

\section{Overall Summary of Genetic Toxicity Evidence}

Summary: Based on the available studies, there is a moderate confidence in the body of evidence that occupational exposure to cancer chemotherapy agents is associated with genetic toxicity. A clear majority of studies reported significant effects of structural CA (22 of 29 studies measuring either percentage of cells with CA and number of CA per 100 cells scored), MN induction (24 of 36 studies measuring either number of micronucleated cells or number of MN per 1,000 cells scored) or DNA damage by comet assay (18 of 21 studies reporting on either percentage of tail DNA, tail length, tail moment and DNA damage (Table F-1). Of the 18 studies that reported on measurements of more than one of the three genetic toxicity assays, 16 reported consistent effects. The initial rating of moderate confidence in the body of evidence for each genetic toxicity assay was not downgraded or upgraded for various factors (Figure 6, Figure 7, and Figure 8). Therefore, the final rating of moderate confidence in the body of evidence directly translates into a rating of a moderate level of evidence that occupational exposure to cancer chemotherapy agents is associated with genetic toxicity as measured by structural CA, MN induction, and DNA damage measured by comet assay.

\section{Overall Risk-of-bias Assessment of Genetic Toxicity Evidence}

Risk-of-bias ratings of individual genetic toxicity studies are available in Figure D-4 and Figure D-5. A general discussion of the common issues for this dataset on the key risk-of-bias 
questions (confounding, exposure characterization, and outcome assessment) is included below. Additional detailed discussion of specific risk-of-bias issues that impact the bodies of evidence for individual measures (i.e., structural CA, MN induction, and DNA damage measured by comet assay) are presented in the following sections.

Risk-of-bias assessment of potential confounders included age, sex, smoking, and co-exposure to other known or suspected genetic toxicants (e.g., anesthetic gases, ionizing radiation, and organic solvents). Most studies (73\%) measuring CA, MN induction or DNA damage measured by comet assay controlled for these potential confounders resulting in definitely low or probably low risk of bias. Among the remaining 27\% of studies, 14 studies (21\%) were identified as probably high risk of bias due to lack of reporting on one or more confounders and modifiers, or the authors did not statistically analyze whether the confounders or modifiers were significantly different between the exposure groups (Figure D-4 and Figure D-5). Six percent of studies (four studies) were rated as definitely high risk of bias for lack of adjustment of confounding or modifying variables, including age, sex, smoking status (Cooke et al. 1991; Ensslin et al. 1997; Kašuba et al. 1999; Kopjar and Garaj-Vrhovac 2001), or genetic toxicant co-exposures [e.g., ionizing radiation (Kašuba et al. 1999)].

Risk-of-bias assessment of the exposure characterization assessed the consistency and reliability of the exposure measures regarding the use of established test methods and whether exposure was assessed in a relevant time-window for development of the outcome. Most of the studies assessing genetic toxicity as probably low risk of bias for exposure characterization because they reported data on work tasks (e.g., preparing drugs, administering drugs, or cleaning up patient excreta) and drug handling (e.g., drug types, frequency of handling, doses, duration of exposure); the data were usually collected by self-reported or interviewer-led questionnaire. Data on work practices, safety equipment availability, and use of personal protective equipment also supported a rating of probably low risk of bias. Three studies characterized exposure using a daily diary in which exposed subjects recorded their work activities and usual events (e.g., accidents), which improved the exposure characterization by allowing for an analysis of number of drug-handling events, handling events for the drugs that were measured, lifetime handling of antineoplastic drugs, job classification, and class of drugs (alkylating, non-alkylating) (Connor et al. 2010) or an incidence rate ratio of CA per the frequency of drug-handling events (based on a daily diary of exposure) (McDiarmid et al. 2010; McDiarmid et al. 2014); these studies were also rated probably low risk of bias because frequency of drug-handling events is considered an acceptable approach for exposure assessment of mixed exposures such as cancer chemotherapy agents (Albertini et al. 2000; Nieuwenhuijsen 2003). Two studies were rated probably high risk of bias because they only reported on the job title of the exposed population, but did not provide any further details characterizing the exposure (Garaj-Vrhovac and Kopjar 1998; Mušák et al. 2006).

Risk-of-bias evaluation of the outcome assessment considered the use of established methods, whether the outcome had been assessed consistently across all groups and whether the outcome assessors had been blinded to the study groups or exposure levels prior to assessing the outcomes. Most of the studies measuring DNA damage as CA, MN induction or DNA damage measured by comet assay were rated probably or definitely low risk of bias for outcome assessment. All studies used standard methods for assessing CA, MN induction, and DNA damage by comet assay (see protocol in Appendix I). Most studies reported the OECD recommended measurements or common measurements for each of these genetic toxicity assays and assessed outcomes consistently across all groups. Most studies also reported blinding of the 
outcome assessor. In addition, the outcome assessment was automated for some studies reporting on MN induction and comet assay; thus, for these studies, there was less of a concern if there was a lack of reporting on whether blinding occurred. Eight studies were also rated probably high risk of bias for lack of details regarding assessment methods for MN (Ensslin et al. 1997) or sample processing for comet assay (Cornetta et al. 2008; Deng et al. 2006; Deng et al. 2005; Mader et al. 2009; Ündeğer et al. 1999; Yang et al. 2002a; Yin et al. 2010). Only one study (Ursini et al. 2006) received a ranking of definitely high risk of bias for outcome assessment for maintaining blood samples at room temperature until processing; this action may have biased the results toward the null because DNA repair enzymes are known to begin working within 30 minutes of sample collection unless they are chilled. The authors reported no difference in tail moment between the exposed and unexposed groups (Ursini et al. 2006).

\section{Overall Confidence Ratings and Level-of-evidence Conclusions for Genetic Toxicity}

Across the three measures of genetic toxicity (increased structural CA, MN induction, and DNA damage measured by the comet assay), there is moderate confidence in the body of evidence that occupational exposure to cancer chemotherapy agents is associated with genetic toxicity. The body of evidence across a total of 66 studies presents a consistent pattern of findings of higher levels of CA, MN induction, and DNA damage measured by comet assay in occupationally exposed subjects. Furthermore, studies that evaluated more than one genetic toxicity assay (e.g., CA, MN, or DNA damage measured by comet assay) were largely consistent in the direction of their results: 14 of 18 studies reported significantly higher levels of DNA damage across multiple assays and two studies reported no difference between exposure groups; two studies reported inconsistent effects across more than one genetic toxicity assay. A potential upgrade in the overall confidence for genetic toxicity was considered based on consistent evidence across multiple effect measures. However, no change was made in the confidence rating because these three indications of genetic toxicity are closely related measures of an effect without support for an independent mechanism. Two of the genetic toxicity assays (CA and MN induction) had the same mechanism of action, and comet assay is a biomarker of effect, which has not yet been associated with any downstream health outcome. The final rating of moderate confidence directly translates into a moderate level of evidence that occupational exposure to cancer chemotherapy agents is associated with genetic toxicity.

\section{Structural Chromosomal Aberrations}

The NTP evaluation identified 29 studies of 26 unique populations reporting structural CA (i.e., breaks, deletions, duplications, translocations, inversions, and rings) resulting from direct DNA breakage, replication on a damaged DNA template, inhibition of DNA synthesis and other mechanisms (e.g., inhibition of topoisomerase II enzymes) in peripheral blood lymphocytes arrested at metaphase. The evaluation focused the level-of-evidence conclusions on studies reporting on the two measurements of CA: percent of cells with CA per 100 cells scored (excluding chromosomal gaps), which are referred to here as percentage of cells with CA (excluding gaps) (Figure D-6), and the number of CA per 100 cells scored (excluding gaps)

(Figure D-7). The percentage of cells with CA (excluding gaps) is a measure of the frequency of cells that exhibited any type of CA, and is a recommended measurement for CA by the OECD (2016a). The number of CA per 100 cells (excluding gaps) scored is a less precise, but commonly reported, measure than percentage of cells with CA (without gaps) because some cells have more than one CA; however, it is included because it is commonly reported in the published literature. Chromosomal and chromatid gaps (also called gaps or achromatic lesions) 
are lightly stained bands in the chromosomes and chromatids and are not accepted as a measure of damage because they occur naturally in apparently unexposed populations, their identification is highly subjective, and the implication of gaps is not known. The OECD Test Guideline 473 for the in vitro mammalian CA test recommends to count the gaps, but not to include them in the measure of total CA (OECD 2016a). NTP also considered studies that measured aberrations in chromosome 5, 7, or 11 to support the CA data because a higher number of aberrations in these three chromosomes have been reported in patients following administration of cancer chemotherapy agents for treatment of cancer [reviewed in McDiarmid et al. (2010)].

\section{Summary}

Based on the available studies, there is a moderate confidence in the body of evidence that occupational exposure to cancer chemotherapy agents is associated with increased structural CA. A clear majority of studies reporting on percentage of cells with CA and number of aberrations per 100 cells scored reported significantly higher effects in individuals with occupational exposure to chemotherapy agents (Table F-1). Eleven of 14 studies reporting on percentage of cells with CA and 11 of 17 studies reporting on the number of aberrations per 100 cells scored reported significantly higher effects on exposed subjects compared to control subjects. Four studies evaluated both CA measurements and reported similar results for each endpoint (ElEbiary et al. 2013; Jakab et al. 2001; Kopjar et al. 2009; Sorsa et al. 1988). Similarly, a preliminary study by Mušák et al. (2006) reported a non-significant ( $p=0.07)$ number of CA in healthcare workers in Slovakia, which was corroborated by a larger study of the same population that reported significantly higher percentage of cells with CA compared to unexposed subjects (Mušák et al. 2013). Furthermore, two studies evaluating CA in chromosomes 5, 7, and 11 also support higher number of CA for individuals with occupational chemotherapy exposure. Specifically, samples from the same population of nursing and pharmacy personnel reported a significantly higher number of CA on chromosome 5 following occupational exposure to alkylating (McDiarmid et al. 2010) as well as non-alkylating agents compared to unexposed subjects (McDiarmid et al. 2014). The initial rating of moderate confidence in the body of evidence was not downgraded or upgraded for various factors (Figure 6). Therefore, the final rating of moderate confidence in the body of evidence directly translates into a rating of a moderate level of evidence that occupational exposure to cancer chemotherapy agents is associated with structural CA.

\section{Overview}

The NTP evaluation identified 29 studies of 26 unique populations reporting structural CA (\% of cells with CA, number of CA/100 cells scored, or incidence rate ratio of CA) resulting from direct DNA breakage, replication on a damaged DNA template, inhibition of DNA synthesis and other mechanisms (e.g., inhibition of topoisomerase II enzymes) in peripheral blood lymphocytes arrested at metaphase. The available epidemiological studies in the body of evidence that evaluated the association between occupational exposure to cancer chemotherapy and CA included: 28 cross-sectional studies of medical personnel as well as chemists, laboratory assistants, and production or manufacturing workers, and one prospective cohort study of pharmacists (Table F-1). The majority of the studies evaluated nurses or pharmacy personnel, while drug production or manufacturing employees were the subject of only three studies. The studies varied greatly in continent where the study was conducted (Europe $=20$ studies, Middle East $=$ five studies, North America = three studies, and Asia = one study), size (from 12 to 594 subjects), and length of time in job position (>6 months to 25 years). Some of the studies also 
measured other DNA damage endpoints, such as MN induction or comet assay (see following sections for discussion of these other health outcomes).

\section{Evidence Synthesis}

The evidence synthesis for percentage of cells with CA (excluding gaps) was based on 14 studies (Table F-1), including one prospective cohort study of pharmacists (Roth et al. 1994) and 13 cross-sectional studies evaluating nurses, pharmacists, physicians, and other health workers as well as studies evaluating employees of drug production and manufacturing facilities (Anwar et al. 1994; Burgaz et al. 2002; El-Ebiary et al. 2013; Hola et al. 1988; Jakab et al. 2001; Kopjar et al. 2009; Medková 1990; Mušák et al. 2013; Nikula et al. 1984; Pohlová et al. 1986; Rubeš et al. 1998; Sessink et al. 1994a; Sorsa et al. 1988). The results show a consistent increase in CA as measured by an increase in the percentage of cells with CA in occupationally exposed subjects. Eleven of the 14 studies reported significantly higher percentage of cells with CA in exposed subjects relative to controls (Figure D-6). Of the three studies reporting no difference between exposed and unexposed subjects, a study of exposed nurses in Turkey reported a non-significant higher percentage of cells with CA compared to unexposed, and exposure was a significant factor in the analysis of variance (Burgaz et al. 2002). The remaining two studies attributed the lack of effect on CA to the availability of safety equipment and use of personal protective equipment by the exposed subjects. A single prospective cohort of pharmacists in Finland reported no difference between exposed subjects and controls (Roth et al. 1994); the authors reported that all exposed subjects used personal protective equipment and prepared the drugs in a vertical laminar flow safety cabinet in an isolated negative-pressured laboratory, separate from other work areas. Sorsa et al. (1988) attributed the lack of difference in percentage off cells with CA to improvements in the proper exposure prevention practices; however, the controls in the factory had higher number of CA than controls from outside the factory.

The evidence synthesis for the number of CA per 100 cells scored (excluding gaps) was based on 17 cross-sectional studies of nurses, pharmacists, and physicians handling cytostatic agents as well as workers in production and manufacturing of these agents (Table F-1) (Bouraoui et al. 2011; Cavallo et al. 2005; Cooke et al. 1991; El-Ebiary et al. 2013; Grummt et al. 1993; Jakab et al. 2001; Kopjar et al. 2009; Krepinsky et al. 1990; Mahmoodi et al. 2017; Milković-Kraus and Horvat 1991; Moretti et al. 2015; Mušák et al. 2006; Sorsa et al. 1988; Stücker et al. 1986; Testa et al. 2007; Thulin et al. 1995; Yang et al. 2002b). The results show a consistent increase in CA as measured by the number of CA per 100 cells in occupationally exposed subjects. Eleven of the 17 cross-sectional studies reported that occupational exposure to cancer chemotherapy agents resulted in a significantly higher number of CA per 100 cells scored (Figure D-7). Of the six studies that did not report a significant association for CA, a study of health workers in Slovakia reported that exposed subjects tended $(\mathrm{p}=0.07)$ to have a higher number of CA than controls (Mušák et al. 2006); the authors subsequently published a larger study of the same population that found a significantly higher percentage of cells with $\geq 1$ CA (Mušák et al. 2013). Two studies of drug production workers in Finland (Sorsa et al. 1988) and Sweden (Thulin et al. 1995) reported similar number of CA compared to unexposed workers; the authors stated that employees wore appropriate personal protective equipment and used safety equipment (e.g., personal fresh-air devices, gas-protective suits). Cooke et al. (1991) attributed the lack of difference between exposed pharmacists and nurses and the control subjects in the United Kingdom to the appropriate use of personal protective equipment and safety equipment (i.e., biological safety cabinets), and the fact the exposed nurses were only responsible for 
administering, but not preparing, the cancer chemotherapy doses. In a study of nurses in France, Stucker et al. (1986) suggested that the lack of effect on the number of CA may be due to less frequent handling of cancer chemotherapy agents in the pneumology department of this study, relative to studies that examine medical staff working in oncology departments. Finally, Krepinsky et al. (1990) suggested that the variability in the dose and frequency of handling of cancer chemotherapy agents among the exposed nurses in may have contributed to the observation of no difference compared to unexposed nurses.

Further support for an association between occupational exposure to cancer chemotherapy agents and induction of CA was provided by McDiarmid and colleagues, who examined the number of aberrations at chromosomes 5, 7, and 11 in a group of nurses, pharmacists, and pharmacy technicians in three university-based hospital cancer centers in the United States (McDiarmid et al. 2010; McDiarmid et al. 2014). The exposed subjects were asked to maintain a 6-week daily diary of the types and frequency of cancer chemotherapy drugs they handled as well as any unusual events (e.g., accidents). The data were presented as incidence rate ratios (IRR) that described the association between the number of CA predicted by the model at the select drughandling frequencies compared with the number of CA at zero drug-handling events (controls). Significantly higher IRRs of aberrations were observed in chromosome 5 as the number of handling events increased for either alkylating agents (McDiarmid et al. 2010) or non-alkylating agents (e.g., anthracycline antibiotics, biologics, antimetabolites) (McDiarmid et al. 2014). The IRRs of aberrations at chromosome 7 and 11 were also increased in a dose-related manner for either alkylating agents or non-alkylating agents, but did not reach statistical significance (McDiarmid et al. 2010; McDiarmid et al. 2014).

\section{Risk-of-bias Assessment}

Risk of bias was assessed for all 29 included studies reporting on percentage of cells with CA, number of CA per 100 cells scored, (Figure D-8 and Figure D-9) and number of aberrations on chromosome 5, 7, or 11 (data not graphed).

Most studies were rated probably low risk of bias for confounding because they evaluated the key confounders and addressed differences in age, sex, smoking status, and possible co-exposure to other genetic toxicants (e.g., ionizing radiation). One study was rated definitely high risk of bias for confounding because the authors did not adjust for age although they reported that controls were significantly older than exposed pharmacists, which could bias the results toward the null (Cooke et al. 1991); the authors reported no difference in number of CA/100 cells. Nine studies receiving a rating of probably high risk of bias for confounding due to either lack of adjustment for confounding variables or insufficient information on how confounders were assessed (Hola et al. 1988; Jakab et al. 2001; Medková 1990; Mušák et al. 2006; Rubeš et al. 1998; Sessink et al. 1994a; Stücker et al. 1986; Thulin et al. 1995; Yang et al. 2002b). Lack of adjustment for confounders did not appear to influence the outcome for one study; thus, it was rated as probably low risk of bias for confounding (Nikula et al. 1984). Specifically, a study of nurses from Finland reported that 6 of 16 unexposed subjects were exposed to other chemicals (e.g., organic solvents) while exposed subjects were not similarly co-exposed (Nikula et al. 1984); the co-exposure did not appear to influence the results toward the null as the exposed subjects had a significantly greater percentage of cells with CA.

Most studies were rated probably low risk of bias for exposure characterization because exposure was based on job title in combination with work tasks (e.g., preparation and/or administration of 
cancer chemotherapy agents, handling patient excreta) and drug handling (e.g., frequency of drug handling, doses administered). Many studies also collected and reported on the level of safety protection available (e.g., biological safety cabinet, isolated room for drug preparation) and personal protective equipment used (e.g., gloves, masks, aprons). Two studies were ranked probably low risk of bias for exposure characterization because the authors further characterized exposure by requiring exposed participants to maintain a 6-week daily diary of the agents used, the frequency of handling each agent as well as any usual events (e.g., accidents) (McDiarmid et al. 2010; McDiarmid et al. 2014), which allowed the authors to analyze the data number of handling events. Biomonitoring further supported a rating of probably low risk of bias because a majority of the exposed subjects (eight of 12 exposed drug manufacturing workers) had levels of cyclophosphamide in their urine above the LOD, while urine samples from all control subjects were below the LOD (Sessink et al. 1994a). Three other studies reporting on biomonitoring of urine samples did not provide further support for a rating of probably low risk of bias rating because two studies reported very few of the urine samples had levels of the agent(s) above the LOD (Cavallo et al. 2005; Moretti et al. 2015), and another study detected cyclophosphamide in the urine samples 12 of the 12 exposed subjects tested, but did not test samples from unexposed subjects (Burgaz et al. 2002).

For outcome assessment, all studies included in the evidence synthesis followed a standard protocol and most studies included blinding of outcome assessors to the exposure status of the participants resulting in a rating of probably low risk of bias. Five studies did not report whether there was blinding for outcome assessment [two studies measuring percentage of cells with CA (Hola et al. 1988; Rubeš et al. 1998) and three studies reporting on the number of CA/100 cells (Krepinsky et al. 1990; Stücker et al. 1986; Yang et al. 2002b)], which resulted in a rating of probably high risk of bias for outcome assessment.

\section{Confidence Ratings and Level-of-evidence Conclusions}

The initial rating of moderate confidence in the body of evidence for CA, including percentage of cells with CA and number of CA per 100 cells scored, was not downgraded or upgraded for various factors (Figure 6). The final rating of moderate confidence in the body of evidence did not change when NTP excluded the study with definitely high risk of bias for confounding (Cooke et al. 1991). 


\begin{tabular}{|c|c|c|c|c|c|c|c|c|c|c|c|}
\hline \multirow[b]{2}{*}{$\begin{array}{l}\text { Initial Confidence for Each } \\
\text { Body of Evidence } \\
\text { (\# of Studies) }\end{array}$} & \multicolumn{5}{|c|}{$\begin{array}{l}\text { Factors Decreasing Confidence } \\
\text { (“-"” if no concern; “ } \downarrow ” \text { if serious } \\
\text { concern to downgrade confidence) }\end{array}$} & \multicolumn{5}{|c|}{$\begin{array}{c}\text { Factors Increasing Confidence } \\
\text { ("“_" if not present; “ } \uparrow " \text { if sufficient to } \\
\text { upgrade confidence) }\end{array}$} & \multirow[b]{2}{*}{$\begin{array}{l}\text { Final } \\
\text { Confidence } \\
\text { Rating }\end{array}$} \\
\hline & 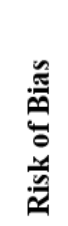 & 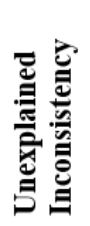 & 苞 & 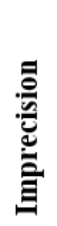 & 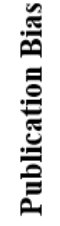 & 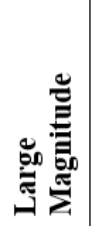 & 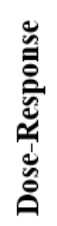 & 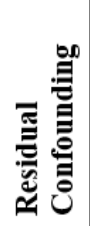 & $\begin{array}{l}\vec{U} \\
\frac{3}{2} \\
\frac{n}{n} \\
0 \\
0\end{array}$ & $\stackrel{\dot{\Xi}}{0}$ & \\
\hline \multicolumn{12}{|l|}{$\%$ Cells with CA $^{\mathbf{b}}$} \\
\hline $\begin{array}{l}\text { Initial Moderate } \\
\text { ( } 1 \text { cohort [prospective], } \\
13 \text { cross-sectional) }\end{array}$ & - & - & - & - & - & - & - & - & - & - & Moderate \\
\hline \multicolumn{12}{|c|}{ Number of $C A / 100$ cells scored ${ }^{c}$} \\
\hline $\begin{array}{l}\text { Initial Moderate } \\
\text { ( } 1 \text { cohort [prospective], } \\
12 \text { cross-sectional) }\end{array}$ & - & - & - & - & - & - & - & - & - & - & Moderate \\
\hline \multicolumn{12}{|c|}{ Number of aberrations on chromosome 5,7 or $11 / 100$ cells scored ${ }^{d}$} \\
\hline $\begin{array}{l}\text { Initial Moderate } \\
\text { ( } 2 \text { cross-sectional })\end{array}$ & - & - & - & - & - & - & - & - & - & - & Moderate \\
\hline
\end{tabular}

Figure 6. Confidence in the Body of Evidence for Structural Chromosomal Aberrations (Excluding Gaps)

anitial confidence of cross-sectional studies of genetic toxicity started as moderate due to the determination that knowledge of exposure status would not influence the results of the CA assays.

b\% cells with CA references: (Anwar et al. 1994; Burgaz et al. 2002; El-Ebiary et al. 2013; Hola et al. 1988; Jakab et al. 2001; Kopjar et al. 2009; Medková 1990; Mušák et al. 2013; Nikula et al. 1984; Pohlová et al. 1986; Roth et al. 1994; Rubeš et al. 1998; Sessink et al. 1994a; Sorsa et al. 1988).

'Number of CA references: (Bouraoui et al. 2011; Cavallo et al. 2005; Cooke et al. 1991; El-Ebiary et al. 2013; Grummt et al. 1993; Jakab et al. 2001; Kopjar et al. 2009; Krepinsky et al. 1990; Mahmoodi et al. 2017; Milković-Kraus and Horvat 1991; Moretti et al. 2015; Mušák et al. 2006; Sorsa et al. 1988; Stücker et al. 1986; Testa et al. 2007; Thulin et al. 1995; Yang et al. 2002b).

${ }^{\mathrm{d}}$ Number of aberrations on chromosome 5, 7, or 11 references (presented as incidence rate ratios): (McDiarmid et al. 2010; McDiarmid et al. 2014).

\section{Micronucleus Induction}

The NTP evaluation identified 35 studies of 32 unique populations reporting MN induction and focused the level-of-evidence conclusions on studies reporting on the number of micronucleated cells per 1,000 cells scored (also called number of cells with micronuclei (MN)) and the number of MN per 1,000 cells scored. This assay detects $\mathrm{MN}$ in the cytoplasm of interphase cells, which may arise from acentric chromosome fragments or whole chromosomes that cannot migrate to the poles during cell division. Micronucleus induction signify structural or numerical chromosomal alterations that have been transmitted to daughter cells. The number of micronucleated cells per 1,000 cells scored is the most precise measure and is recommended by the 2014 OECD Testing Guidelines for the In Vitro Micronucleus Test (OECD 2016b). NTP also evaluated the number of MN per 1,000 cells scored because it is a frequently reported endpoint; however, this measure is considered slightly less reliable because a cell can have more than one micronucleus. Micronuclei induction was evaluated in two cells types: binucleated lymphocytes and buccal cells. The NTP evaluation used the abbreviation MN for both the singular (micronucleus) and plural forms (micronuclei) of the word, as applicable. 


\section{Summary}

Based on the available studies, there is moderate confidence in the body of evidence that occupational exposure to cancer chemotherapy is associated with greater MN induction. Eleven of 13 studies measuring the number of cells with $\mathrm{MN}$ and 16 of 26 studies reporting on the number of MN per 1,000 cells scored reported significantly greater effects in exposed subjects compared to unexposed subjects (Table F-1). Four studies evaluated both measurements of MN and reported similar results for each endpoint (Deng et al. 2006; Deng et al. 2005; Kašuba et al. 1999; Kopjar et al. 2009). In addition, three sets of studies-(Boughattas et al. 2010; Bouraoui et al. 2011), (Garaj-Vrhovac and Kopjar 1998; Kašuba et al. 1999), and (Sorsa et al. 1988; Yager et al. 1988)_evaluated the three similar or overlapping populations and reported consistent results with the exception of Yager et al. (1988), which included an extra group of oncology nurses with a high number of MN compared to Sorsa et al. (1988). The initial rating of moderate confidence in the body of evidence was not downgraded or upgraded for various factors (Figure 7). The final confidence rating in the body of evidence directly translates into a rating of moderate level of evidence that occupational exposure to cancer chemotherapy agents is associated with MN induction.

\section{Overview}

The available epidemiological studies in the body of evidence that evaluated the association between occupational exposure to cancer chemotherapy and MN induction included 34 crosssectional studies of medical personnel as well as chemists, laboratory assistants, and production or manufacturing workers; and one prospective cohort study of pharmacists (Table F-1). Most studies evaluated nurses, while drug production or manufacturing employees were the least studied (four studies). The studies varied greatly in country of origin (Europe $=20$, Middle East $=5$, Asia $=4$, South America $=3$, and North and Central America $=2$ ), size (from $n=20$ to 156), and length of time in job position (1 month to 30 years). Some of the studies also measured other DNA damage endpoints, such as CA or comet assay (see other sections of Results and Evidence Synthesis for discussion of these other health outcomes).

\section{Evidence Synthesis}

The evidence synthesis for the number of cells with MN per 1,000 cells scored was based on 13 studies (Table F-1): one prospective cohort study of pharmacists (Roth et al. 1994) and 12 crosssectional studies evaluating nurses, doctors, pharmacists and production workers (Anwar et al. 1994; Boughattas et al. 2010; Buono-Michel et al. 2000; Deng et al. 2006; Deng et al. 2005; Kašuba et al. 1999; Kopjar et al. 2009; Machado-Santelli et al. 1994; Maluf and Erdtmann 2000b; Rodríguez-Montero et al. 2016; Rombaldi et al. 2009; Yang et al. 2002b). The results show a consistently higher number of cells with MN per 1,000 cells scored in exposed subjects relative to controls (Figure D-10). Eleven of the 13 studies reported a significantly higher number of cells with $\mathrm{MN}$ in exposed subjects relative to controls. Of the two studies reporting negative a lack of effect on MN, a study of nurses and pharmacists in Brazil reported no difference in the number of cells with MN compared to unexposed subjects following an adjustment in the work schedule to limit exposure and the hiring of additional employees (Maluf and Erdtmann 2000b); four years prior, this population reported a significantly higher number of cells with MN in exposed subjects than controls. A prospective cohort of pharmacists in Finland reported no difference between exposed subjects and controls (Roth et al. 1994), which the authors attribute to the use of personal protective equipment and the availability of a vertical 
laminar flow safety cabinet for drug preparation in an isolated negative-pressured laboratory, separate from other work areas.

The evidence synthesis for the number of MN/1,000 cells scored was based on 26 cross-sectional studies evaluating 23 unique populations of nurses, doctors, pharmacists and drug production workers (Table F-1) (Bolognesi et al. 2005; Bouraoui et al. 2011; Burgaz et al. 1999; Cavallo et al. 2005; Cornetta et al. 2008; Deng et al. 2006; Deng et al. 2005; El-Ebiary et al. 2013; Ensslin et al. 1997; Garaj-Vrhovac and Kopjar 1998; Harris et al. 1992; Hessel et al. 2001; Kašuba et al. 1999; Kevekordes et al. 1998; Kopjar et al. 2009; Ladeira et al. 2014; Laffon et al. 2005; Mahmoodi et al. 2017; Moretti et al. 2015; Moretti et al. 2013; Mrđanović et al. 2012; Pilger et al. 2000; Rekhadevi et al. 2007; Sorsa et al. 1988; Thiringer et al. 1991; Yager et al. 1988). The results show a consistent increase in $\mathrm{MN}$ induction as measured by an increase in the number of MN/1,000 cells scored in exposed subjects relative to control subjects (Figure D-11 and Figure D-12). Sixteen of 26 studies reported a significantly higher number of MN/1,000 cells scored in exposed subjects compared to controls. Of the nine studies reporting no effect of exposure, a study of exposed medical workers including nurses, pharmacy personnel, one physician, and one orderly in Germany did not observe any difference in the number of $\mathrm{MN}$ compared to unexposed office workers (Hessel et al. 2001); frequency of drug handling of the exposed subjects was not reported. Moretti et al. (2013) reported no significant differences in the number of MN between exposed workers in a hospital in Italy responsible for preparation, transport, administration, and disposal of cancer chemotherapy agents unexposed blood donors, but did report a significantly higher level of DNA damage measured by tail intensity in exposed subjects. Eight studies reported a lack of effect of occupational exposure on the number of MN in nursing and pharmacy personnel that used personal protective equipment and/or prepared drugs in vertical laminar flow safety cabinets (Bolognesi et al. 2005; Cavallo et al. 2005; Ensslin et al. 1997; Harris et al. 1992; Laffon et al. 2005; Moretti et al. 2013; Pilger et al. 2000; Thiringer et al. 1991). Harris et al. (1992) also observed that nurses with low and moderate exposure that used gloves $<100 \%$ of the time had significantly higher number of MN compared to nurses using gloves $100 \%$ of the time. Finally, one study of drug production and manufacturing workers, and associated laboratory technicians in Finland reported a non-significant higher number of MN compared to unexposed workers following exposure safety protection improvements (Sorsa et al. 1988). Of note, two studies reported contrasting results for number of MN from studies overlapping in the population under study. While Sorsa et al. (1988) reported no effect on the number of MN, Yager et al. (1988) tested the same population and included an extra group of exposed workers in their study (oncology nurses) who had a higher number of MN than the other exposed groups; the authors combined the data for all the exposed workers and observed a significantly higher number of MN when analyzed by an ANOVA of the log-transformed data (Yager et al. 1988).

\section{Risk-of-bias Assessment}

Risk-of-bias assessment was conducted on all studies included in the evidence synthesis for MN induction presented number of cells with MN per 1,000 cells scored and number of MN per 1,000 cells scored (Figure D-13 and Figure D-14). Similar to the body of evidence for CA, most studies on $\mathrm{MN}$ induction were rated probably low risk of bias for confounding (e.g., appropriate confounders were considered and adjusted when necessary) and outcome assessment (e.g., the assessors were generally blinded to treatment), as well as probably high risk of bias for exposure 
characterization due to exposure classification by job position without confirmation of exposure from biomonitoring measurements.

Most studies were rated as probably low risk of bias for confounding. Two studies were rated as definitely high risk of bias for confounding (Ensslin et al. 1997; Kašuba et al. 1999). In a study reporting no difference in the number of MN per 1,000 cells scored between exposed pharmacists ( $\mathrm{n}=13$ subjects) and controls ( $\mathrm{n}=13$ subjects) in Germany, Ensslin et al. (1997) did not adjust for reported differences in sex and smoking status between exposed and control subjects (women: controls $=11$ of 13 and exposed $=8$ of 13; smoking: controls $=4$ of 13 and exposed $=1$ of 13), which could have biased the results toward the null. Kašuba et al. (1999) did not adjust for a higher number of exposed subjects co-exposed to ionizing radiation (7 of 20) compared to controls exposed (1 of 16), which could have biased the results away from the null; this study observed a significantly higher number of MN per 1,000 cells scored in exposed nurses relative to control subjects. Four studies did not report confounding variables or the methods used to collect data on confounding (Deng et al. 2006; Garaj-Vrhovac and Kopjar 1998; Harris et al. 1992; Yager et al. 1988; Yang et al. 2002b). One study was rated probably low risk of bias even though the authors did not adjust for significant covariates. Hessel et al. (2001) did not directly adjust the results for a significantly greater number of men in the controls versus the exposed pharmacy personnel in Germany, which could have biased the results away from the null: however, there was no difference in the number of MN/1,000 cells scored between exposure groups.

Similar to studies assessing CA, most studies were rated probably low risk of bias for exposure characterization because exposure was based on job title in combination with work tasks (e.g., preparation and/or administration of cancer chemotherapy agents, handling patient excreta) and drug handling (e.g., frequency of drug handling, doses administered). Many studies also collected and reported on the level of safety protection available (e.g., biological safety cabinet, isolated room for drug preparation) and personal protective equipment used (e.g., gloves, masks, aprons). Urine biomonitoring results did not provide further support for a rating of probably low risk of bias for five studies testing urine samples from both exposed and unexposed subjects (Bolognesi et al. 2005; Cavallo et al. 2005; Ensslin et al. 1997; Moretti et al. 2015; Pilger et al. 2000) as none of the studies reported positive samples for a majority of the exposed subjects. Another MN study that measured cyclophosphamide in urine of exposed and unexposed occupationally exposed hospital, pharmacy, and medical personnel reported no correlation between a positive urine sample $\geq$ LOD versus urine samples that measured $<$ LOD (Hessel et al. 2001). Two studies reported positive biomonitoring results in a majority of exposed subjects, but did not test unexposed subjects (Burgaz et al. 1999; Rekhadevi et al. 2007); specifically, cyclophosphamide was above the LOD in urine samples of 19 of 25 (Burgaz et al. 1999) and 42 of 52 exposed nurses (Rekhadevi et al. 2007).

Regarding risk of bias for outcome assessment, 11 studies did not report blinding and the authors did not respond to NTP's request for information (Bolognesi et al. 2005; Boughattas et al. 2010; Buono-Michel et al. 2000; Cornetta et al. 2008; Deng et al. 2006; Deng et al. 2005; Ensslin et al. 1997; Garaj-Vrhovac and Kopjar 1998; Kašuba et al. 1999; Yager et al. 1988; Yang et al. 2002b). 


\section{Confidence Ratings and Level-of-evidence Conclusions}

The initial rating of moderate confidence in the body of evidence for $\mathrm{MN}$, including number of cells with MN/1,000 cells scored and number of MN/1,000 cells scored, was not downgraded or upgraded for various factors (Figure 7). The final rating of moderate confidence in the body of evidence did not change when NTP excluded the studies with definitely high risk of bias for confounding.

\begin{tabular}{|c|c|c|c|c|c|c|c|c|c|c|c|}
\hline \multirow[b]{2}{*}{$\begin{array}{l}\text { Initial Confidence for Each } \\
\text { Body of Exidence }{ }^{\mathrm{a}} \\
\text { (\# of studies) }\end{array}$} & \multicolumn{5}{|c|}{$\begin{array}{l}\text { Factors Decreasing Confidence } \\
\text { ("-"” if no concern; " } \downarrow " \text { if serious } \\
\text { concern to downgrade confidence) }\end{array}$} & \multicolumn{5}{|c|}{$\begin{array}{l}\text { Factors Increasing Confidence } \\
\text { ("-" if not present; "个" if sufficient } \\
\text { to upgrade confidence) }\end{array}$} & \multirow[b]{2}{*}{$\begin{array}{l}\text { Final } \\
\text { Confidence } \\
\text { Rating }\end{array}$} \\
\hline & 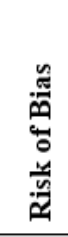 & 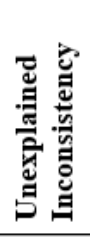 & 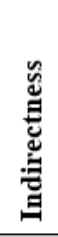 & 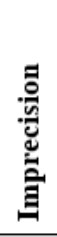 & 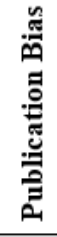 & 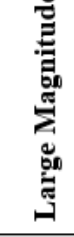 & 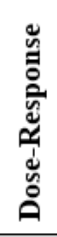 & 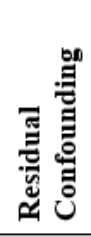 & 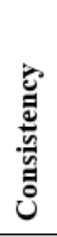 & ఏँ & \\
\hline \multicolumn{12}{|c|}{ Number of cells with micronuclei $(\mathrm{MN}) / 1,000$ cells scored ${ }^{b, c}$} \\
\hline $\begin{array}{l}\text { Initial Moderate } \\
\text { (1 cohort [prospective], } \\
12 \mid \text { cross-sectional) }\end{array}$ & - & - & - & - & - & - & - & - & - & - & Moderate \\
\hline \multicolumn{12}{|c|}{ Number of MN/1,000 cells scored d,e } \\
\hline $\begin{array}{l}\text { Initial Moderate } \\
\text { ( } 26 \text { cross-sectional) }\end{array}$ & - & - & - & - & - & - & - & - & - & - & Moderate \\
\hline
\end{tabular}

Figure 7. Confidence in the Body of Evidence for Micronucleus Induction

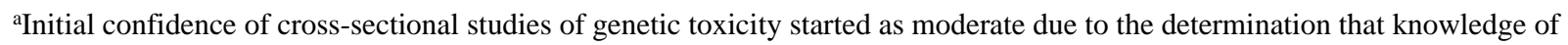
exposure status would not influence the results of the MN assays.

${ }^{b}$ Number of cells with MN/1,000 binucleated lymphocytes scored references: (Anwar et al. 1994; Boughattas et al. 2010; BuonoMichel et al. 2000; Deng et al. 2006; Deng et al. 2005; Kašuba et al. 1999; Kopjar et al. 2009; Maluf and Erdtmann 2000b;

Rombaldi et al. 2009; Roth et al. 1994; Yang et al. 2002b).

${ }^{\mathrm{c} N u m b e r}$ of cells with MN/1,000 buccal cells scored references: (Machado-Santelli et al. 1994; Rodríguez-Montero et al. 2016). dNumber of MN/1,000 binucleated lymphocytes scored references: (Bolognesi et al. 2005; Bouraoui et al. 2011; Burgaz et al. 1999; Cavallo et al. 2005; Cornetta et al. 2008; Deng et al. 2006; Deng et al. 2005; El-Ebiary et al. 2013; Ensslin et al. 1997; Garaj-Vrhovac and Kopjar 1998; Harris et al. 1992; Hessel et al. 2001; Kašuba et al. 1999; Kevekordes et al. 1998; Kopjar et al. 2009; Ladeira et al. 2014; Laffon et al. 2005; Mahmoodi et al. 2017; Moretti et al. 2015; Moretti et al. 2013; Mrđanović et al. 2012; Pilger et al. 2000; Rekhadevi et al. 2007; Sorsa et al. 1988; Thiringer et al. 1991; Yager et al. 1988).

eNumber of MN/1,000 buccal cells scored references: (Bolognesi et al. 2005; Burgaz et al. 1999; Cavallo et al. 2005; Rekhadevi et al. 2007).

\section{DNA Damage Measured by the Comet Assay}

The NTP evaluation identified 21 studies of 18 unique populations reporting DNA damage measured by the comet assay. The level-of-evidence conclusions were based on studies reporting the following four measurements: the percent of tail DNA (also called \% tail intensity), tail length $(\mu \mathrm{m})$, tail moment, and DNA damage index. The percent of tail DNA corresponds to the fraction of DNA intensity of the comet tail relative to total intensity (head plus tail); this comet assay measurement is recommended for use by the OECD Testing Guideline for In Vivo Mammalian Comet Assay (OECD 2016c). Tail length is the maximum length of DNA migration and is measured either from the center of the head or the leading edge of the head to the tip of the tail. Tail moment may be measured as extent tail moment (\% tail DNA multiplied by tail length) or Olive tail moment (\% tail DNA multiplied by tail moment length; tail moment is calculated as 
length from the center of the head to the center of the tail). Tail length and tail moment are considered acceptable measurements if an image analysis software system is used to score slides. However, tail moment is not a recommended measurement for comet assay due to variability in the methods to calculate tail moment (OECD 2016c) and the fact that tail moment has no units attached to it. Another commonly reported measurement of comet assay was a DNA damage index, which was a sum of the cells with DNA damage weighted by the severity of damage; this method is not among the recommended measured by the OECD (2016c). The majority of studies evaluated either lymphocytes or leukocytes. For accurate comet assay results, the OECD guidelines state that samples must be kept ice-cold, or frozen, until processing. Otherwise, enzymes will act to repair any damaged DNA and may cause the test results to be inaccurate.

\section{Summary}

Based on the available studies, there is a moderate confidence in the body of evidence that occupational exposure to cancer chemotherapy agents is associated with increased DNA damage measured by the comet assay. Five of seven studies reporting on percent of tail DNA, 10 of 11 studies reporting on tail length $(\mu \mathrm{m})$, and five of five studies reporting a DNA damage index reported significantly higher rates of DNA damage compared to unexposed subjects (Table F-1). Only two of seven studies reporting on tail moment reported significantly higher levels of DNA damage in exposed subjects than controls; however, tail moment is not recommended measurement by the OECD due to variability in methods to calculate tail moment (OECD 2016c). Seven studies evaluated two or more tail measurements (Connor et al. 2010; Deng et al. 2006; Deng et al. 2005; Kopjar and Garaj-Vrhovac 2001; Sasaki et al. 2008; Villarini et al. 2011; Yin et al. 2010); however, only three studies reported consistent results between each endpoint (Connor et al. 2010; Kopjar and Garaj-Vrhovac 2001; Yin et al. 2010); tail length and tail moment were the most common endpoint with no difference compared to unexposed subjects. The initial rating of moderate confidence in the body of evidence was not downgraded or upgraded for various factors (Figure 8). The final rating of moderate confidence in the body of evidence directly translates into a rating of moderate level of evidence that occupational exposure to cancer chemotherapy agents is associated with DNA damage measured by comet assay.

\section{Overview}

The available epidemiological studies in the body of evidence that evaluated the association between occupational exposure to cancer chemotherapy and DNA damage measured by comet assay included one prospective cohort study (Mader et al. 2009) and 20 cross-sectional studies of medical personnel as well as chemists, laboratory assistants, and production or manufacturing workers); and one prospective cohort study of pharmacists (Table F-1). Most the studies evaluated nursing and pharmacy personnel, while drug production or manufacturing employees were the least studied (only two studies). The studies originated from 10 countries: Italy $(\mathrm{n}=5)$, China $(n=4)$, Brazil $(n=2)$, Croatia $(n=2)$, Japan $(n=2)$, Turkey $(n=2)$, Austria $(n=1)$, India $(n=1)$, Portugal $(n=1)$, and United States $(n=1)$. The studies varied in number of participants (from $n=15$ to 167), and length of time in job position (2.4 months to 23 years). Some of the studies also measured other DNA damage endpoints, such as CA or MN induction (see other sections of Results and Evidence Synthesis for discussion of these other health outcomes).

\section{Evidence Synthesis}

The evidence synthesis for percent of tail DNA was based on the seven cross-sectional studies of nurses, pharmacists, and health workers handling cytostatic agents (Table F-1) (Buschini et al. 
2013; Connor et al. 2010; Cornetta et al. 2008; Kopjar and Garaj-Vrhovac 2001; Moretti et al. 2013; Villarini et al. 2011; Yin et al. 2010). The results show a consistently higher rate of DNA damage measured by comet assay as measured by percent of tail DNA in exposed subjects relative to controls (Figure D-15). Five of seven studies reported that exposed subjects had a higher percent of tail DNA than controls (Cornetta et al. 2008; Kopjar and Garaj-Vrhovac 2001; Moretti et al. 2013; Villarini et al. 2011; Yin et al. 2010). The observation of no effect of exposure in a study of nurses in Italy may have been due to the lack of chilling the blood samples upon collection (Buschini et al. 2013); a lack of chilling may allow DNA repair enzymes to correct DNA damage thus biasing toward the null. A study of nurses, pharmacists, and pharmacist technicians in the United States reported a lack of effect of exposure, which the authors attributed to adequate provision of safety equipment and use of personal protective equipment by the exposed personnel (Connor et al. 2010).

The evidence synthesis for tail length was based on 11 cross-sectional studies reporting on nurses, physicians, and production workers handling cancer chemotherapy agents (Table F-1). The results show a consistently higher rate of DNA damage measured by comet assay as measured by tail length in exposed subjects relative to controls (Figure D-16). Ten of 11 studies reported significantly greater tail length $(\mu \mathrm{m})$ in exposed subjects relative to controls (Deng et al. 2006; Deng et al. 2005; Kopjar and Garaj-Vrhovac 2001; Kopjar et al. 2009; Laffon et al. 2005; Rekhadevi et al. 2007; Sasaki et al. 2008; Yang et al. 2002a; Yin et al. 2010; Yoshida et al. 2006). One study of health workers in Italy reported no difference in tail length between exposed and control subjects (Villarini et al. 2011); however, the authors reported significantly greater percent of tail DNA in exposed subjects than controls from the same study.

The evidence synthesis for tail moment was based on seven cross-sectional studies reporting on nurses, pharmacists, physicians, and production workers working with cancer chemotherapy agents (Table F-1). The results for tail moment showed a less consistent effect of higher DNA damage measured by comet assay as were observed for percent of tail DNA or tail length, with only two studies reporting significantly greater mean tail moment compared to unexposed subjects (Kopjar and Garaj-Vrhovac 2001; Yin et al. 2010) (Figure D-17). Of the five studies reporting a lack of effect on tail moment, Connor et al. (2010) also reported no effect on percent of tail DNA or tail length, which the authors attributed to proper use of personal protective equipment and other safe handling practices. Tail moment appeared to be a less sensitive DNA damage endpoint for three other studies that reported significant effects of occupational exposure on percent of tail DNA (Villarini et al. 2011) or tail length (Deng et al. 2006; Deng et al. 2005). Ursini et al. (2006) reported that exposed nurses had similar tail moment values to unexposed nurses, which they attributed to the use of personal protective equipment and the use of specialized pharmacy to use the drugs, which meant that the nurses were not involved in drug preparation. However, the authors did not chill blood samples prior to processing, which may have contributed to the lack of difference in tail moment between exposed subjects and controls (Ursini et al. 2006). Another study reported no difference in the log-transformed values of mean tail moment between exposed nurses and unexposed nurses (Sasaki et al. 2008).

The evidence synthesis for studies reporting a DNA damage index measured by comet assay was based on one longitudinal cohort study spanning 9 months (Mader et al. 2009) and four crosssectional studies reporting on nurses and pharmacists (Izdes et al. 2009; Maluf and Erdtmann 2000b; Rombaldi et al. 2009; Ündeğer et al. 1999) (Table F-1). The results for DNA damage measured as DNA damage index by comet assay were consistent with all five studies reporting 
significantly higher number of cells with DNA damage than controls (Figure D-18). All five studies evaluated the proportion of DNA fragments in the tail; however, the number of cells evaluated, the number of categories, and the data presentation differed among the studies (see footnote of Figure D-18 for more details).

\section{Risk-of-bias Assessment}

Risk-of-bias assessment was conducted on all included studies reporting on DNA damage measured by comet assay including measurements of percent of tail DNA, tail length, tail moment, and DNA damage index (Figure D-19 and Figure D-20). Like the body of evidence for CA and MN, most studies on DNA damage measured by comet assay were rated probably low risk of bias for confounding (e.g., appropriate confounders were considered and adjusted when necessary), exposure assessment, and outcome assessment (e.g., the assessors were generally blinded to treatment).

Regarding confounding, a study reporting significantly higher tail length in exposed physicians and nurses in Croatia versus controls was rated definitely high risk of bias because there was no adjustment for sex even though all exposed subjects were females compared to $50 \%$ of control subjects, which could bias the results away from the null (Kopjar and Garaj-Vrhovac 2001). One study was rated probably high risk of bias: a cross-sectional study reporting a greater tail length in exposed nurses in China versus controls did not report the gender of the participants [presumed female] and did not adjust for age differences between controls were younger than exposed subjects (average age 18 years versus 29 years), which could have biased the results away from the null (Yang et al. 2002a).

All studies were rated probably low risk of bias for exposure characterization because exposure was based on job title in combination with work tasks (e.g., preparation and/or administration of cancer chemotherapy agents, handling patient excreta) and drug handling (e.g., frequency of drug handling, doses administered). Many studies also collected and reported on the level of safety protection available (e.g., biological safety cabinet, isolated room for drug preparation) and personal protective equipment used (e.g., gloves, masks, aprons). Urine biomonitoring was conducted in both exposed and unexposed subjects of three studies (Connor et al. 2010; Ursini et al. 2006; Villarini et al. 2011); none of the studies reported positive samples for a majority of the exposed subjects and the biomonitoring was consistent with no difference in comet assay measurements compared to unexposed subjects of these studies (Connor et al. 2010; Ursini et al. 2006). One study conducted biomonitoring in exposed subjects only and reported that cyclophosphamide was above the LOD in urine samples of 42 of 52 exposed nurses (Rekhadevi et al. 2007).

For outcome assessment, two studies reporting no difference in DNA damage measured by comet assay compared to unexposed subjects reported that blood samples were maintained at room temperature after sample collection, which resulted in definitely high risk of bias [tail moment in (Ursini et al. 2006)] or probably high risk of bias [\% tail DNA in (Buschini et al. 2013)]; author responded to query for information about methods). For six studies, a rating of probably high risk of bias for blinding for lack of reporting on blinding was upgraded to probably low risk of bias due to use of automated methods for outcome assessment (Connor et al. 2010; Cornetta et al. 2008; Sasaki et al. 2008; Ursini et al. 2006; Yin et al. 2010; Yoshida et al. 2006). Seven studies did not report the methods used for the comet assay or sample processing details and, thus, were assigned probably high risk of bias (Cornetta et al. 2008; Deng 
et al. 2006; Deng et al. 2005; Mader et al. 2009; Ündeğer et al. 1999; Yang et al. 2002a; Yin et al. 2010).

\section{Confidence Ratings and Level-of-evidence Conclusions}

The initial rating of moderate confidence in the body of evidence for DNA damage measured by the comet assay, including percent tail DNA, tail length and tail moment, and DNA damage index, was not upgraded or downgraded for factors affecting confidence in the body of evidence (Figure 8). The final rating of moderate confidence in the body of evidence did not change when NTP excluded data on tail moment due to known heterogeneity across laboratories in calculating the outcome measurement (OECD 2016c).

\begin{tabular}{|c|c|c|c|c|c|c|c|c|c|c|c|}
\hline \multirow[b]{2}{*}{$\begin{array}{l}\text { Initial Confidence for Each } \\
\text { Body of Exidence } \\
\text { (\# of Studies) }\end{array}$} & \multicolumn{5}{|c|}{$\begin{array}{l}\text { Factors Decreasing Confidence } \\
\text { (“-”" if no concern; “ } \downarrow " \text { if serious } \\
\text { concern to downgrade confidence) }\end{array}$} & \multicolumn{5}{|c|}{$\begin{array}{c}\text { Factors Increasing Confidence } \\
\text { (“-” if not present; “个” if sufficient } \\
\text { to upgrade confidence) }\end{array}$} & \multirow[b]{2}{*}{$\begin{array}{l}\text { Final } \\
\text { Confidence } \\
\text { Rating }\end{array}$} \\
\hline & 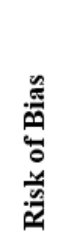 & 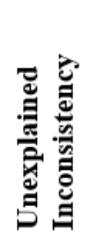 & 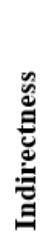 & 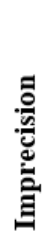 & 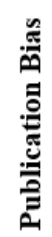 & 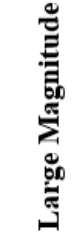 & 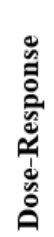 & 葛 & 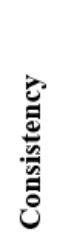 & 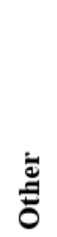 & \\
\hline \multicolumn{12}{|l|}{ \% Tail DNA } \\
\hline $\begin{array}{l}\text { Initial Moderate } \\
\text { ( } 7 \text { cross-sectional) }\end{array}$ & - & - & - & - & - & - & - & - & - & - & Moderate \\
\hline \multicolumn{12}{|l|}{ Tail Lengthe } \\
\hline $\begin{array}{l}\text { Initial Moderate } \\
\text { (11 cross-sectional) }\end{array}$ & - & - & - & - & - & - & - & - & - & - & Moderate \\
\hline \multicolumn{12}{|l|}{ Tail Moment ${ }_{n}^{d}$} \\
\hline $\begin{array}{l}\text { Initial Moderate } \\
\text { ( } 7 \text { cross-sectional) }\end{array}$ & - & - & - & - & - & - & - & - & - & - & Moderate \\
\hline \multicolumn{12}{|c|}{ DNA Damage, Manual Scoring and Binninge } \\
\hline $\begin{array}{l}\text { Initial Moderate } \\
\text { ( } 1 \text { cohort [prospective], } \\
4 \text { cross-sectional })\end{array}$ & - & - & - & - & - & - & - & - & - & - & Moderate \\
\hline
\end{tabular}

Figure 8. Confidence in the Body of Evidence for DNA Damage Measured by the Comet Assay

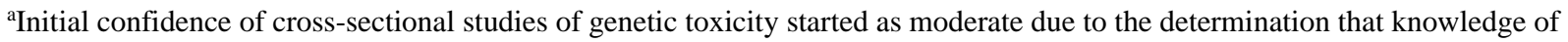
exposure status would not influence the results of the comet assays.

b\% tail DNA: (Buschini et al. 2013; Connor et al. 2010; Cornetta et al. 2008; Kopjar and Garaj-Vrhovac 2001; Moretti et al. 2013; Villarini et al. 2011; Yin et al. 2010).

'Tail length: (Deng et al. 2006; Deng et al. 2005; Kopjar and Garaj-Vrhovac 2001; Kopjar et al. 2009; Laffon et al. 2005; Rekhadevi et al. 2007; Sasaki et al. 2008; Villarini et al. 2011; Yang et al. 2002a; Yin et al. 2010; Yoshida et al. 2006).

${ }^{d}$ Tail moment: (Connor et al. 2010; Deng et al. 2006; Deng et al. 2005; Kopjar and Garaj-Vrhovac 2001; Sasaki et al. 2008; Ursini et al. 2006; Yin et al. 2010).

eDNA damage assessed by manual scoring and binning: (Izdes et al. 2009; Mader et al. 2009; Maluf and Erdtmann 2000b; Rombaldi et al. 2009; Ündeğer et al. 1999).

\section{Evidence of Occupational Exposure to Cancer Chemotherapy Agents}

The NTP evaluation reviewed the literature reporting on environmental monitoring of the workplace (e.g., surfaces, air) and biomonitoring surveillance (e.g., urine or blood levels) of 
cancer chemotherapy agents in occupationally exposed personnel to determine the prevalence of contamination of the work environment and internal doses to personnel. The studies are available in Appendix I in Supplemental Table 1 (Environmental Monitoring) and Supplemental Table 2 (Biomonitoring). The following details were collected per study: citation, publication year, study country, study setting, sampling year (date samples were collected), samples taken (type of cancer chemotherapy agent measured), sensitivity of contaminant measurements (limit of detection or limit of quantitation), and summary of results. Guidelines for safe handling of cancer chemotherapy agents were first issued in the 1980s by professional trade organizations and federal agencies of several countries, including the United States (OSHA 1986). Since that time, additional guidelines have been written and revised for handling cancer chemotherapy agents and other hazardous drugs by 13 different countries [reviewed in Bernabeu-Martinez et al. (2018)]. One of NTP's original goals for Objective 2 of the evaluation was to identify whether levels of contamination had declined in the years since implementation of the 1986 guidelines in the United States and other countries. However, NTP found this objective was challenging to address because the data were not always expressed in the same increment of measure, the same type of sample (e.g., air versus wipe sample, urine versus blood), the same chemotherapy agent (e.g., cyclophosphamide versus 5-fluorouracil), or the same type of sample location (e.g., worktop of the biological safety cabinet versus patient drug administration area). For this reason, NTP selected cyclophosphamide as a surrogate cancer chemotherapy agent and compared studies that measured the same type of sample with the same increments of measure, regardless of the country of origin of the study.

\section{Environmental Monitoring}

The NTP evaluation identified 104 studies reporting on environmental monitoring ranging in publication dates from 1983 to 2017 and years sampled from 1981 to 2016 (Supplemental Table 1 in Appendix I). The studies tested for contamination on a range of samples, including air, cleaning water, gloves, vials, and work surfaces (e.g., floor, biological safety cabinet, and storage shelf). The studies tested and reported on 20 different cancer chemotherapy agents; cyclophosphamide was the most frequently evaluated agent (83 studies). Other agents frequently evaluated in environmental monitoring studies included: ifosfamide ( $\mathrm{n}=42$ ), 5-fluorouracil ( $\mathrm{n}=35)$, methotrexate $(\mathrm{n}=27)$, and platinum (a marker for platinum-containing agents, including cisplatin, carboplatin, and oxaliplatin; $n=18$ ). The remaining agents were evaluated in seven or fewer studies (Supplemental Table 1 in Appendix I). The environmental monitoring studies were conducted in 18 countries. Thirty-nine percent of the studies were conducted in the United States or Canada (41 studies; United States, $\mathrm{n}=22$, and Canada, $\mathrm{n}=18$, and one study reporting on both countries), while 52\% of the studies were conducted in Europe (54 studies; including studies in Italy ( $n=11)$, the Netherlands $(n=9)$, and Germany $(n=7)$. In addition, there were three environmental monitoring studies each from Australia and Japan, and two studies each conducted in Brazil and Iran.

A majority of the environmental monitoring studies (96\%; 100/104 studies) reported that one or more samples (e.g., wipe test, air filters) tested above the LOD for any of the cancer chemotherapy agents evaluated (Supplemental Table 1 in Appendix I). To observe possible trends in contamination of the workplace by cancer chemotherapy agents over time, NTP focused on the studies testing for cyclophosphamide because this agent was measured and reported in a majority of the studies available over a wide range of sampling years (1983-2016). 
Specifically, NTP evaluated the data for two areas of the drug preparation room that are frequently monitored: the workspace of the biological safety cabinet and the floor in front of the biological safety cabinet; 26 studies were available that reported the maximal level of surface contamination for these two areas. The maximum levels detected in the workspace of the biological safety cabinet ranged from $<$ LOD to $1,193 \mathrm{ng} / \mathrm{cm}^{2}$ for the workspace of the biological safety cabinet ( $\mathrm{n}=22$ studies) and $<$ LOD to $402.5 \mathrm{ng} / \mathrm{cm}^{2}$ for the floor in front of the biological safety cabinet (24 studies) (Figure 9). The highest levels of cyclophosphamide surface contamination were reported for these two areas in 2004-2005 (maximum levels for workspace were 17.17-44.17 ng/cm² and floor were 26.8-100 ng/cm²) (Acampora et al. 2005; Sessink et al. 2011; Sessink et al. 2013). The frequency of studies reporting wipe samples with maximum levels above $1.5 \mathrm{ng} / \mathrm{cm}^{2}$ was lower in 2011-2016 (38\%, 3/8 studies) compared to 2005-2010 (67\%, 4/7 studies) based on workspace measurements. However, for the floor samples, the frequency of studies reporting wipe samples with maximum levels above $1.5 \mathrm{ng} / \mathrm{cm}^{2}$ appears to have remained the same over time (2011-2016: 29\%, 2/7 studies versus 2005-2010: 25\%, 4/12).

Workspace

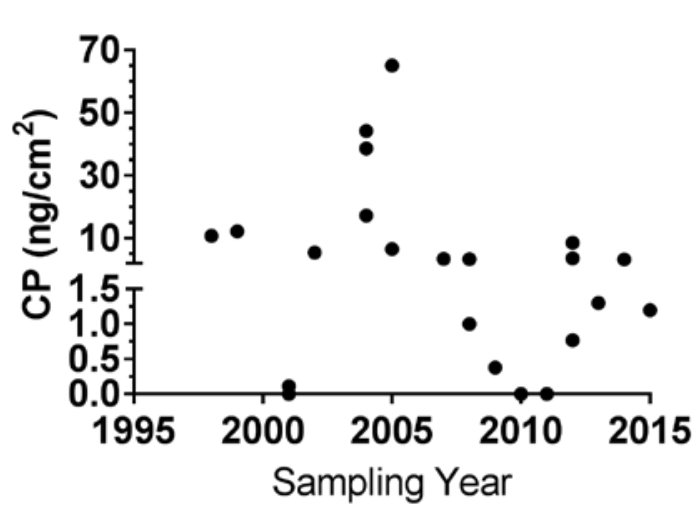

Floor

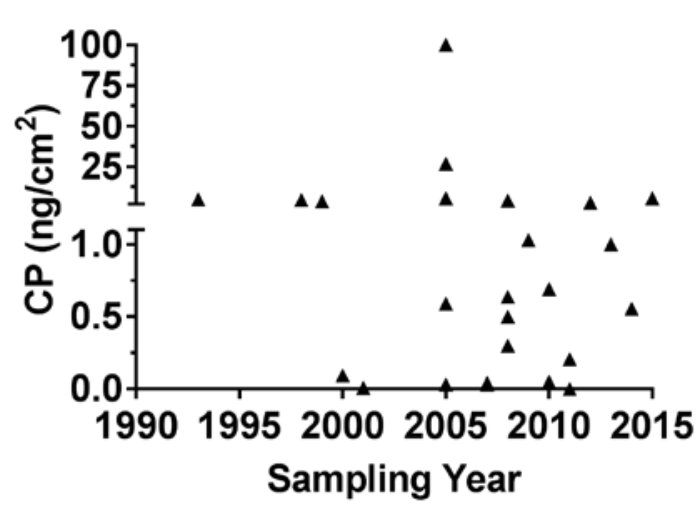

Figure 9. Maximum Levels of Cyclophosphamide Contamination Reported for the Biological Safety Cabinet Workspace and Floor in Front of the Biological Safety Cabinet from 1993 to 2016

Data included 26 studies reporting maximum levels of cyclophosphamide contamination of the workspace of biological safety cabinet (also called hood) or isolator and the floor in front of this space ( $=19)$ : (Acampora et al. 2005; Berruyer et al. 2015; Bigelow et al. 2009; Bussières et al. 2012; Chu et al. 2012; Connor et al. 1999; Couch and dePerio 2011; Couch et al. 2012; Couch and West 2012; Crauste-Manciet et al. 2005; Hedmer et al. 2008; Janes et al. 2015; Martins et al. 2008 ; McDevitt et al. 1993; Merger et al. 2014; Minoia et al. 1998; Odraska et al. 2014; Odraska et al. 2011; Schmaus et al. 2002; Sessink et al. 2013; Siderov et al. 2009; Touzin et al. 2009; Vandenbroucke and Robays 2001; Wick et al. 2003) Six references reported on floor samples only (Bigelow et al. 2009; Hedmer et al. 2008; Odraska et al. 2014; Odraska et al. 2011; Schmaus et al. 2002; Siderov et al. 2009) and one reference measured biological safety cabinet samples only (Schierl et al. 2016).

\section{Biomonitoring}

The NTP evaluation identified 82 studies reporting biomonitoring of occupationally exposed workers. The studies included data collected in 1982-2016 (Supplemental Table 2 in Appendix I). Only four biomonitoring studies were conducted prior to or including 1986, when safe handling guidelines were first published (OSHA 1986). The studies reported on a total of 16 cancer chemotherapy agents or their metabolites with cyclophosphamide (54 studies) as the most frequently evaluated agent. Other agents frequently evaluated in biomonitoring studies include: ifosfamide $(n=19)$, platinum (as a marker of platinum-containing agents: cisplatin, carboplatin, 
and oxaliplatin; $n=21)$, methotrexate $(n=12)$, alpha-fluoro-beta-alanine (a metabolite of 5fluorouracil; $n=9)$, and epirubicin $(n=8)$. The remaining agents were evaluated in six or fewer studies: azathioprine, daunorubicin, docetaxel, doxorubicin, etoposide, gemcitabine, idarubicin, mitomycin C, paclitaxel, and pemetrexed. The biomonitoring studies were conducted in 17 countries. Most of the studies $(64 \%, n=54)$ were conducted in Europe. Only $13 \%$ were conducted in the United States (six studies) and Canada (five studies), 16 studies in Asia, and one study in Africa. Biomonitoring studies primarily evaluated urine samples, although six studies also measured blood levels only and another five studies measured both blood and urine levels of these agents.

Most studies reported the detection of one or more cancer chemotherapy agent in blood or urine of occupationally exposed individuals (89\%; $n=73$ of 82 total studies) (Supplemental Table 2 in Appendix I). Of the 19 studies that also evaluated control (also called unexposed) subjects, seven studies reported samples above the level of detection for both exposed and control subjects (Ensslin et al. 1994a; Fransman et al. 2007b; Konate et al. 2011; Kopp et al. 2013a; Pilger et al. 2000; Ramphal et al. 2015; Sessink et al. 1994a; Sessink et al. 1994b), which suggested that environmental contamination that may be the issue. For example, in a small study of pharmacy personnel, Ramphal et al. (2015) reported that the urine samples both exposed and unexposed pharmacy staff tested positive for cyclophosphamide on the day they received training on use of the urine sample kits in the oncology laboratory (i.e., the five unexposed pharmacy staff were exposed by entering the oncology pharmacy). In a follow-up evaluation, urine samples tested positive for cyclophosphamide in one unexposed subject who reentered the oncology laboratory, while no agent was detected in samples of four unexposed subjects who did not re-enter the area; it was determined that there was surface contamination of cyclophosphamide and methotrexate both before and after cleaning (Ramphal et al. 2015). Nurses not handling cancer chemotherapy agents, but employed in an outpatient clinic where they were administered, had detectable levels of cyclophosphamide in their urine (Fransman et al. 2007b). Of note, Hon et al. (2015) evaluated employees in five acute care sites and one cancer center in Canada and observed that workers not responsible for handling the agents in the drug administration area (e.g., volunteers, oncologist, nurse aides, and dieticians) had the highest number of positive urine samples for cyclophosphamide. The authors reported there was no correlation between urinary concentration levels and known contact with the agents; however, the urine samples with the highest levels of cancer chemotherapy agents were from employees handling the highest doses, and even higher levels of cancer chemotherapy agents were detected in urine samples from employees who did not receive training in handling these drugs versus those employees who received the training.

To observe possible trends in the number of positive subjects reported in biomonitoring efforts, NTP focused on the studies testing for cyclophosphamide because of the number of studies available (54 studies) and the wide range of years tested (1984-2015). Of the 46 studies reporting the data on a per-subject (e.g., nurse) basis, cyclophosphamide was detected in 35 studies. Twelve studies reported no detection of the agent (Figure 10), including three studies with subjects that tested positive in previous sampling years (Sabatini et al. 2012; Sottani et al. 2010; Yoshida et al. 2013). An additional six studies evaluating the data on a per-sample (not per-subject) basis also detected cyclophosphamide in more than one sample (Fransman et al. 2007a; Peelen et al. 1999; Pethran et al. 2003; Sottani et al. 2008; Turci et al. 2011; Turci et al. 2002) (data not graphed). Thus, biomonitoring studies demonstrated that workers continue to be exposed to cancer chemotherapy agents in the workplace. It is observed that the LOD of 
cyclophosphamide in urine has decreased over time; the LODs for the studies in Figure 10 spanned a range from 0.1 to $1.0 \mathrm{ng} / \mathrm{mL}$ in 1992, which decreased to range from 0.009 to $0.1 \mathrm{ng} / \mathrm{mL}$ from 2011 to 2016 . The prevalence of positive biomonitoring samples may be due to more sensitive analytical methods, as well as a higher volume of patients administered cancer chemotherapy agents.

\section{Other Occupationally Exposed Populations}

The potential for cancer chemotherapy exposure has increased as options for administration of treatment and the patients receiving these agents have expanded. One newer technique for administering chemotherapy directly to the target organ involves surgery. A study that evaluated platinum environmental surface levels and biomonitoring following a heated intraperitoneal perioperative chemotherapy (HIPEC) procedure using oxaliplatin detected platinum on both layers of the surgeon's gloves (external gloves: 16,284.3 to 24,857.1 ng; internal gloves: 24.3 to $83.2 \mathrm{ng}$ ) and a small amount on the surgeon's bare hands (2.0-2.2 ng); however, the urine samples of the surgeon and staff were negative for the agent (Konate et al. 2011). Nursing assistants and housekeeping at home care and nursing centers as well as staff at industry laundry centers maybe exposed to cancer chemotherapy agents through their duties of cleaning patients and emptying pot/urinals, and laundering exposed bed linens, respectively (Meijster et al. 2006). Although less frequently reported, laboratory animal care and veterinary staff are also potentially exposed to cancer chemotherapy agents due to companion animals administered cancer chemotherapy agents for the treatment of cancer. Sessink et al. (1993) reported detection of cyclophosphamide (700 ng) in 1 of 87 urine samples taken from four animal care workers handling mice injected with cyclophosphamide. In a study from Canada, Hall et al. (2013) estimated that $22-24 \%$ of veterinarians and $20-22 \%$ of veterinary technicians are exposed to cancer chemotherapy agents. Exposure may occur not only in preparing and administering these agents (Couch et al. 2013; Janssens et al. 2015a), but cancer chemotherapy agents have been also been detected blood samples (Knobloch et al. 2010), urine, feces, saliva, and cerumen (ear wax) of dogs several days following treatment (Janssens et al. 2015b). In addition to occupational exposure, family members and friends of patients receiving cancer chemotherapy agents may also be exposed to these agents. A cross-sectional study of family members of patients administered cyclophosphamide for breast cancer reported that 21\% of the 243 urine samples (representing six of the 10 family members) tested positive for the agent (Yuki et al. 2015). The level of cyclophosphamide detected was similar in the urine samples of 4 of 6 of the family members (101.5-252 ng) compared to patients administered the drug (108.3-181.5), while urine samples from unexposed subjects were < LOD for cyclophosphamide. Individuals sharing a common bathroom or a higher amount of time in a common space with the cancer patient had the highest levels of cyclophosphamide in their urine (Yuki et al. 2015). 


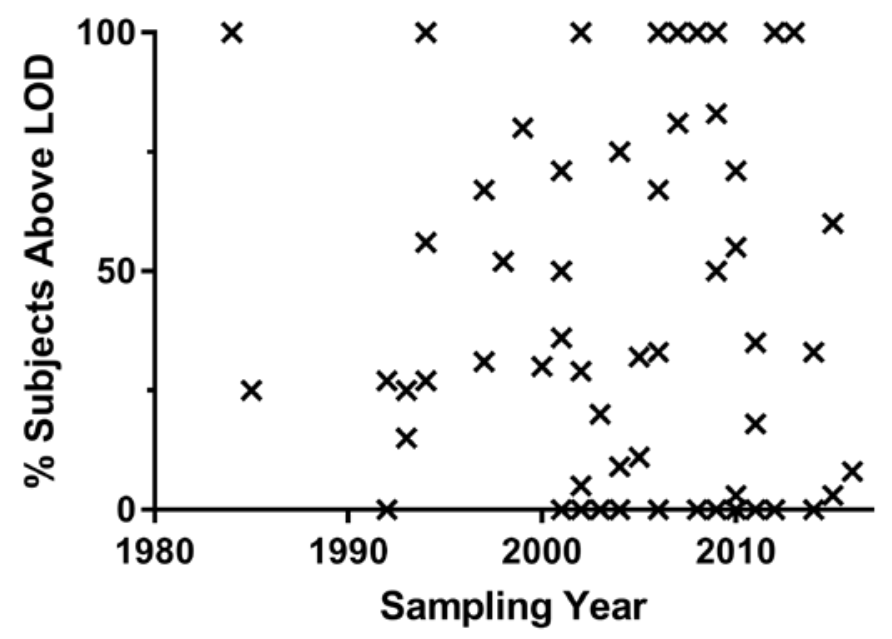

Figure 10. Percent of Workers with Cyclophosphamide Detected Above the Level of Detection in Urine Samples Displayed by the Year Samples Were Collected (1985-2016)

Includes only studies reporting the number of positive subjects or studies for which the number of positive subjects could be determined from the number of samples reported (i.e., one urine sample per subject). Data from 46 studies, including: (Bolognesi et al. 2005; Burgaz et al. 1999; Burgaz et al. 2002; Canal-Raffin et al. 2016; Connor et al. 2010; Ensslin et al. 1997; Ensslin et al. 1994b; Evelo et al. 1986; Hama et al. 2012; Hama et al. 2009; Hedmer et al. 2008; Hirst et al. 1984; Hon et al. 2015; Karahalil and Ilter Akkoyunlu 2004; Kiffmeyer et al. 2002; Korczowska and Jankowiak-Gracz 2013; Maeda et al. 2010; Minoia et al. 1998; Miyake et al. 2013; Moretti et al. 2015; Nyman et al. 2007; Poupeau et al. 2017; Ramphal et al. 2015; Ramphal et al. 2014; Rekhadevi et al. 2007; Sabatini et al. 2012; Schreiber et al. 2003; Sessink et al. 1992a; Sessink et al. 1992b; Sessink et al. 1994a; Sessink et al. 2015; Sessink et al. 1994c; Sessink et al. 1997b; Sessink et al. 1993; Sottani et al. 2010; Sottani et al. 2012; Sugiura et al. 2011a; Sugiura et al. 2011b; Tanimura et al. 2009; Turci et al. 2011; Turci et al. 2002; Vandenbroucke and Robays 2001; Villarini et al. 2011; Wick et al. 2003; Yoshida et al. 2013; Yoshida et al. 2009; Yuki et al. 2015; Ziegler et al. 2002). 


\section{Discussion}

Based on a systematic review of the literature, NTP concludes that there is a moderate level of evidence that occupational exposure to cancer chemotherapy agents is associated with spontaneous abortion and genetic toxicity. The review identified several studies evaluating other health outcomes; however, there were few studies available for most organ systems and there was heterogeneity in the outcomes assessed for many of them, which limited the ability to compare results between studies. Despite the issuance of guidelines for safe handling of cancer chemotherapy, increased usage of personal protective equipment, and improved safety containment equipment, studies of environmental monitoring and biomonitoring demonstrated that workplace contamination and exposure of workers continues to occur even as recently as from 2011 to 2016.

A clear majority of the genetic toxicity studies in the NTP evaluation reported significantly higher levels of structural CA, MN induction, and DNA damage measured by the comet assay. Fourteen of the 18 studies evaluating more than one assay reported significantly greater DNA damage in exposed subjects relative to unexposed subjects. Most of the studies reporting no effect of occupational exposure also documented the availability of containment devices (e.g., ducted biological safety cabinets), isolated rooms for drug preparation and/or the high usage of personnel protective equipment (e.g., aprons, gloves, and respiratory protection) among occupationally exposed personnel. The NTP conclusion of a moderate level of evidence for genetic toxicity reflects moderate confidence in the body of evidence including consideration of study quality in terms of risk of bias and other factors that could decrease or increase confidence. The inclusion of risk-of-bias assessment in this evaluation is more extensive than previous study quality assessment methods (e.g., "quality” scoring by consideration of study features) used in three recent meta-analyses of the genetic toxicity and occupational exposure (Roussel et al. 2017; Villarini et al. 2016; Zare Sakhvidi et al. 2016). A recent meta-analysis of studies reported that occupational exposure to cancer chemotherapy agents was associated with a significantly higher occurrence of MN induction compared to unexposed subjects (Villarini et al. 2016). The authors reported an overall meta-estimate for $\mathrm{MN}$ frequency of 1.67 (95\% CI $=1.41-1.98$; analyzed as a random ratio of means) for exposed subjects compared to control subjects, and the analysis included studies measuring both the number of cells with $\mathrm{MN}$ as well as studies measuring the number of MN (Villarini et al. 2016). Another recent systematic review and meta-analysis of healthcare providers handling cancer chemotherapy agents reported a significantly higher DNA damage index (measured by the comet assay) compared to unexposed workers (Zare Sakhvidi et al. 2016); the authors reported an overall meta-estimate of DNA damage index of 1.93 (95\% $\mathrm{CI}=1.15-2.71$; analyzed as an estimated standardized mean difference). Finally, a meta-analysis of healthcare workers occupationally exposed to cancer chemotherapy agents reported a significantly higher rate of cells with CA or number of CA per cells scored compared to unexposed subjects (Roussel et al. 2017). The overall test results for a pooled standardized mean difference were 1.006, $\mathrm{z}=4.25, \mathrm{p}<0.0001$ (Roussel et al. 2017). The overall results of these three meta-analyses corroborated the NTP's level-of-evidence conclusions for genetic toxicity in the current evaluation.

Many cancer chemotherapy agents are known or reasonably anticipated to be human carcinogens based on the induction of secondary cancers in patients therapeutically treated with these drugs for cancer and toxicological studies in laboratory rodents (IARC 1966; 1980; 1981; 1990; 2000; 
2012; Mistry et al. 2005; NTP 2016 ; Pedersen-Bjergaard 2005). Therapy-related acute myeloid leukemia (t-AML) is the most frequently diagnosed secondary cancer and was recently reported to occur 4.70 times more frequently in patients previously administered cancer chemotherapy than in the general population of the United States in 1975-2008 (Morton et al. 2013). Secondary cancers of the bladder, lung, skin, and uterus are also reported to occur more frequently following administration of cancer chemotherapy agents to patients or laboratory animal models, including following treatment with alkylating agents, antimetabolites, and topoisomerase II inhibitors (IARC 2012). The data on cancer incidence evaluated in the NTP monograph were inadequate to reach level-of-evidence conclusions (three studies) due to the availability of only one or two studies and a low number of cases. Two risk assessments estimated the risk of leukemia, skin cancer, and bladder cancer in nurses handling cyclophosphamide based on their average measurements of dermal exposure (Fransman et al. 2014) or urine levels (Sessink et al. 1995) and cancer incidence in experimental animal studies or primary and secondary cancers in patients receiving cyclophosphamide as a treatment for cancer. Both risk assessments reported that the estimated cancer incidence based on average occupational exposure was within the prohibitory risk level (i.e., no risk higher than 100 per million a year); however, the authors point out that these assessments were based on only one cancer chemotherapy agent when many are used, and there are individuals exposed to higher than average levels, which could increase their risk of developing cancer.

Adverse reproductive outcomes were the second most frequently evaluated health outcome category associated with occupational exposure to cancer chemotherapy. There is strong biological support and concern for potential adverse reproductive health outcomes because many cancer chemotherapy agents are known teratogens or embryo toxicants as identified in toxicology studies in animals and observational studies of patients receiving these drugs for treatment of cancer and other health conditions (NTP 2016; Shepard and Lemire 2004). The NTP monograph observed a higher occurrence of spontaneous abortions in nursing and pharmacy personnel responsible for handling cancer chemotherapy agents compared to controls. One recent review and meta-analysis (Dranitsaris et al. 2005) and a systematic review (Quansah and Jaakkola 2010) of healthcare providers working with cancer chemotherapy agents reached similar conclusions that exposure was associated with spontaneous abortions. The NTP conclusions extended the evaluation of spontaneous abortion by these two reviews (Dranitsaris et al. 2005; Quansah and Jaakkola 2010) by assessing the quality of the studies (Dranitsaris et al. (2005) did not include this step), and by the addition of studies published since the previous reviews (Lawson et al. 2012; Zhang et al. 2016). While other reproductive outcomes were reported in the published literature, there was insufficient evidence to reach level-of-evidence conclusions on the body of literature for a variety of reasons. For example, while 12 studies assessed congenital malformations, only three studies limited exposure to the first trimester and the results were inconsistent. Several studies reported no association of stillbirth with occupational exposure to cancer chemotherapy agents. While the lack of an association of stillbirth with occupational exposure to cancer chemotherapy was contrary to the observed higher rate of stillbirth in women administered cancer chemotherapy during pregnancy reviewed collectively in a recent NTP monograph (NTP 2013), the results on stillbirth risk with occupational exposure to cancer chemotherapy appear to be consistent with the results of two pregnancy registry studies of women exposed to cancer chemotherapy for treatment of cancer compared to pregnancies of unexposed cancer patients (Cardonick et al. 2010; Van Calsteren et al. 2010). However, it was determined that the body of evidence was inadequate for reaching 
level-of-evidence conclusions due to the inability to reach a high level of confidence needed to determine no effect, given the designs of the available studies (retrospective cohort and crosssectional studies) and the lack of dose-response data (see OHAT Handbook for Conducting a Literature-Based Health Assessment [https://ntp.niehs.nih.gov/go/systematic_review]).

\section{Impact of Exposure Protection Efforts}

Several studies suggested that adherence to good practices, implementation of safety containment equipment, and other exposure mitigation efforts can be successful at reducing occupational exposure and, thus, lowering the potential for adverse health outcomes. While studies only measuring environmental monitoring and/or biomonitoring of occupational exposure to cancer chemotherapy agents following improvements to reduce exposure were not systematically reviewed in this report, the studies are briefly mentioned here because of the importance that reducing occupational exposure has in ultimately reducing adverse health outcomes. For example, a study of four hospital pharmacies in Italy from 1998 to 2007 observed decreases in positive urine samples from pharmacy staff and wipe samples of the work environment that coincided with the implementation of safe handling guidelines for handling these drugs in 1999, and the usage of personal protective equipment (e.g., chemotherapy gloves, gowns, hair covers) in the 2000s (Sottani et al. 2010). Reductions in the levels of cancer chemotherapy agents detected in exposed workers' urine samples or environmental monitoring of workspaces where cancer chemotherapy drugs are prepared and/or handled have also been observed with the implementation of a centralized system for the preparation of doses (Sabatini et al. 2012) or closed-system drug transfer devices, which limit the chance of drug spills or the escape of vapor during drug preparation and intravenous drug administration (Sessink et al. 2011; Sessink et al. 2013; Wick et al. 2003; Yoshida et al. 2009).

Of the studies reporting health outcomes that were all systematically reviewed, only one study assessed health outcomes before and after implementation of exposure control methods. The study in China reported a significant reduction in a variety of adverse health outcomes (i.e., incidence of spontaneous abortion, infertility, and hematotoxicity) in oncology nurses 2 years after the implementation of a PIVAS at the hospital, compared to the frequency of these adverse health outcomes at the time the PIVAS was first established (Zhang et al. 2016). In addition, several studies reporting no difference in the number of MN between occupationally exposed employees and controls attributed this lack of effect to the documented usage of personal protective equipment, available biological safety cabinets, and/or centralized units for drug preparation by the occupationally exposed workers (Bolognesi et al. 2005; Cavallo et al. 2005; Ensslin et al. 1997; Harris et al. 1992; Laffon et al. 2005; Pilger et al. 2000; Thiringer et al. 1991).

\section{Limitations of the Evidence Base}

\section{Study Design Limitations}

Recall bias can occur in retrospective cohort studies and case-control studies, and most of the studies reporting on reproductive outcomes or cancer in the NTP monograph were retrospective. Recall bias is most likely when both the exposure and disease status are known at time of study, and it is recommended that the study design does not explicitly reveal the purpose of questions in an interview or survey and/or uses a validated questionnaire (Andrews et al. 2002). For example, 
parents of children with autism associated the condition with exposure to the MMR (mumpsmeasles-rubella) vaccine after this hypothesis was published in 1998; whereas, parents did not associate this exposure with MMR prior to publication of the hypothesis (Andrews et al. 2002). However, studies report that women have accurate recall of pregnancy outcomes (Axelsson 1990; Joffe et al. 1993), and nurses have accurate recall of pregnancy outcomes and health data for many outcomes (Colditz et al. 1986; Colditz et al. 1987). Thus, studies of reproductive health and major disease outcomes in medical personnel are considered of greater reliability despite the retrospective study design (Axelsson 1990). Furthermore, a study of pharmacists demonstrated accurate recall of occupational exposure to cancer chemotherapy agents using a self-reported questionnaire was corroborated by their supervisor (McDiarmid et al. 1992). Many of the studies reporting health outcomes were of the cross-sectional study design, and this study design generally cannot assure that exposure occurred prior to outcome so reverse causation is possible. Following the Hill considerations on causality (which are embedded in the OHAT adaptations of the GRADE approach to assessing confidence in the body evidence used here), there is less confidence that the relationship between exposure and outcome is causal. The initial confidence rating for cross-sectional studies is generally low confidence based on only two features: individual outcomes assessed, and that a comparison group was used. In this evaluation, the cross-sectional studies of genetic toxicity were rated moderate initial confidence, based primarily on the rapidity of the genetic toxicity mechanism (hours to days). Therefore, for genetic toxicity, NTP considered cross-sectional studies to provide sufficiently clear evidence that exposure preceded outcome, based on the fact that the studies measured rapidly developing health outcomes (hours to days versus years), the subjects worked full time the week the samples were drawn, and most subjects had worked from 1 month to a year prior to the study.

\section{Exposure Characterization}

A potential limitation of the exposure characterization of occupational exposure to cancer chemotherapy agents is the nature of the primary source of information on exposure: employment history or historical industrial hygiene data. The utility of this information is improved by use of validated questionnaires regarding work activities (e.g., drugs handled, quantities, frequency) and unusual events (i.e., accidents and needle sticks) that may influence exposure during the study period. In fact, the use of a daily diary of work activities that has been validated to estimate exposure levels has been proposed for use with genetic toxicity outcomes by the World Health Organization's International Programme on Chemical Safety (Albertini et al. 2000). The daily diary has been proposed as the most useful source to construct exposure metrics based on the level of exposure (drugs handled, frequency of handling), use of protective measures (personal protective equipment, ventilation), variances in procedures, and adverse events (Nieuwenhuijsen 2003). Biomonitoring of urine or blood samples for levels of a cancer chemotherapy agent(s) or its metabolites may be helpful to confirm that exposure occurred, but likely only provide confirmation of only the most recent exposure. Environmental monitoring of the work environment (e.g., surface wipes, air samples, dermal pads) is a useful tool to identify contaminated areas, and, thus, the potential for exposure; however, it may or may not relate to actual internal dose due to use of safety equipment (e.g., biological safety cabinets) or personal protective equipment (e.g., gloves, mask, gown). A recent review of swipe tests compared to biomonitoring results did not find a statistically significant correlation (Kibby 2017); however, the authors did observe decreases in the levels of surface contamination detected in wipe samples and urine monitoring following improvements to personal protective equipment and safe 
handling techniques. Another challenge for biomonitoring and environmental monitoring assessments is the fact that most occupational exposures in the medical or veterinary setting are likely to multiple cancer chemotherapy agents, while most monitoring assessments are designed to test one or a handful of agents as surrogates of the total cancer chemotherapy exposure.

\section{Challenges in Evaluating Reproductive Toxicity}

There were many challenges in the outcome assessment of reproductive toxicity associated with occupational exposure to cancer chemotherapy. The occurrence of some pregnancy outcomes, such as major congenital malformations, is relatively rare; thus, large sample sizes are required to detect a difference between exposed and unexposed subjects. Some adverse reproductive health outcomes may be difficult to accurately detect and, thus, may not always be fully documented in the workers' medical records (e.g., early spontaneous abortion). However, several studies have determined that women have accurate recall of pregnancy outcomes, including spontaneous abortion (Axelsson 1990) and time to pregnancy (Cooney et al. 2009). Finally, occupational exposure to cancer chemotherapy agents was not a frequently reported exposure during pregnancy. For example, some studies evaluated the association of fetal loss or congenital malformations with a woman's occupation (Lorente et al. 2000; McDonald et al. 1988b), or occupational exposures to a variety of hazardous chemicals in hospitals (Hemminki et al. 1985; McAbee et al. 1993), the pharmaceutical manufacturing industry (Taskinen et al. 1986), or laboratory work (Taskinen et al. 1994). The total number of workers handling cancer chemotherapy agents in these studies was small and may not be representative of the actual prevalence of fetal loss or congenital malformations in the larger population of exposed workers.

\section{Limitations of the Systematic Review}

There were some limitations of the current systematic review. The NTP evaluation excluded studies that did not include a comparator group due to concerns that knowledge of the outcome may have influenced the association with occupational exposure to cancer chemotherapy; thus, the evaluation did not include 21 studies (e.g., case reports, case series or surveys) that reported on a variety of health outcomes, including acute effects, asthma, cancer, and reproduction. However, these studies could be useful to identify additional health outcomes that could be evaluated in future research. Secondly, the literature search may not have identified all possible studies reporting on health outcomes following occupational exposure to cancer chemotherapy, particularly if journals were not indexed for the databases that NTP searched (i.e., PubMed, Scopus, and Embase). For example, NTP identified one reference reporting on CA and MN induction in peripheral lymphocytes (Ferguson et al. 1988) and another reference reporting on MN induction in buccal cells (Odio et al. 2004) from the reference lists of recently published papers, as NTP completed its evaluation. NTP is confident it has identified the majority of papers published on these genetic toxicity outcomes and have confirmed that the results of the additional two papers reporting on CA and MN induction would not change NTP's level-ofevidence conclusions for these endpoints or genetic toxicity overall. Finally, NTP's literature search may not have captured the entire body of literature on environmental monitoring (e.g., contamination of drug vials, gloves) and biomonitoring studies because the literature search was primarily focused on identifying the health outcome studies. However, NTP is confident its review of this literature provides an accurate characterization of the levels of environmental contamination in the workplace and the prevalence of positive biomonitoring studies for a surrogate exposure (i.e., the commonly used cancer chemotherapy agent cyclophosphamide). 


\section{Research Needs}

The NTP evaluation identified several data gaps and research needs to improve the scientific community's understanding of potential health risks associated with occupational exposure to cancer chemotherapy agents:

1. Expand the range of health outcomes assessed in evaluations of occupational exposure to cancer chemotherapy agents, including bone marrow function (e.g., hematotoxicity), and toxicity of the cardiovascular, kidney, and liver systems. Although some reports of these health outcomes were identified in the NTP evaluation, there was insufficient evidence to evaluate whether or not there is an association with occupational exposure to chemotherapy.

2. Expand the array of workers studied for health effects associated with occupational exposure to cancer chemotherapy agents to include support personnel (e.g., waste collectors, hospital cleaning and laundry staff), home health aides, emergency responders, and veterinary clinic staff. In addition, family caregivers could be studied as exposure to this population has been documented. There were very few studies reporting on the health outcomes or exposure levels of these worker populations, including family caregivers, in the NTP evaluation.

3. Expand the evaluation of health effects associated with occupational exposure to cancer chemotherapy agents to include emerging cancer drug therapies that target specific genes, transcription factors, or signaling molecules. Side effects have been observed in patients following administration of newer targeted drug therapies, such as monoclonal antibodies (Halsen and Kramer 2011) and B-Raf proto-oncogene inhibitors (Sibaud et al. 2013). Most studies in the NTP evaluation focused on health outcomes associated with occupational exposure primarily to antineoplastic agents.

4. Conduct more prospective cohort studies versus retrospective cohort or case-control studies to reduce the potential for recall bias in identifying occupational exposure, especially for endpoints such as cancer. Cross-sectional studies, which were among the most common study design in the NTP evaluation, also present concerns about possible reverse causation. Prospective cohort studies allow for a baseline measure of health outcomes of all subjects in the study to which subsequent health evaluations can be compared.

5. Improve exposure characterization methods:

a. Advance analytical chemistry methods for detection of cancer chemotherapy agents to lower the LOD, allow for simultaneous detection of multiple cancer chemotherapy agent exposures (e.g., most oncology nurses and pharmacists handle multiple agents), and develop techniques to detect metabolites of the agents (e.g., most biomonitoring assays only measure the parent agent). While many of the environmental monitoring studies did evaluate more than one agent, most of the biomonitoring studies assessed only one agent. Those studies that assessed multiple agents tended to evaluate them sequentially. Very few biomonitoring studies (16 of 82 studies) tested for metabolites of cancer chemotherapy agents. 
b. Continue research on ways to effectively use daily diaries that are assessed and validated to estimate exposure levels (e.g., frequency of handling, dose levels, as well as incorporation of information on use of personal protective equipment and safety containment equipment). Although many studies collected information about drugs handled, doses, and frequency of handling, these data were not generally incorporated into the association with the health outcome. In contrast, two studies of the same population that characterized exposure by daily diary allowed for an analysis that considered the individual's health outcome by the individual's drug handling activity (McDiarmid et al. 2010; McDiarmid et al. 2014).

6. Design studies to evaluate the effectiveness of different types of personal protective equipment and other exposure control strategies (e.g., closed-system drug transfer devices, centralized drug preparation units) to reduce occupational exposure (environmental monitoring and biomonitoring) and to reduce possible adverse health outcomes. The impact of exposure control strategies was evaluated in only one study reporting on health outcomes and a handful of environmental monitoring and/or biomonitoring studies reviewed in the NTP monograph. 


\section{Conclusions}

The NTP evaluation concluded that there is a moderate level of evidence for spontaneous abortion and genetic toxicity associated with occupational exposure to cancer chemotherapy agents. Data on other health effects was inadequate to reach level-of-evidence conclusions due to limitations in the body of evidence (e.g., few studies, small case sizes and, in some cases, heterogeneity in the outcomes assayed). Environmental monitoring and biomonitoring studies report that workplace contamination with cancer chemotherapy agents continues to occur, although maximum levels of surface contamination detected have declined. Considering the potential for exposure, the association between exposure and DNA damage in workers, and the widespread use of cancer chemotherapy agents (e.g., veterinary use, home health care), there is a continued need to improve exposure characterization (e.g., improved analytical chemistry methods, daily diaries of drug handling) and health surveillance as well as to identify the most effective safe handling practices and exposure control equipment to reduce potential adverse health outcomes of occupationally exposed personnel. 


\section{References}

Acampora A, Castiglia L, Miraglia N, Pieri M, Soave C, Liotti F, Sannolo N. 2005. A case study: Surface contamination of cyclophosphamide due to working practices and cleaning procedures in two Italian hospitals. Ann Occup Hyg. 49(7):611-618.

http://dx.doi.org/10.1093/annhyg/mei029

Alam SS, Hafiz NA, Abd El-Rahim AH. 2011. Protective role of taurine against genotoxic damage in mice treated with methotrexate and tamoxfine. Environ Toxicol Pharmacol. 31(1):143-152. http://dx.doi.org/10.1016/j.etap.2010.10.001

Albertini RJ, Anderson D, Douglas GR, Hagmar L, Hemminki K, Merlo F, Natarajan AT, Norppa H, Shuker DE, Tice R et al. 2000. IPCS guidelines for the monitoring of genotoxic effects of carcinogens in humans. International Programme on Chemical Safety. Mutat Res. 463(2):111-172. http://dx.doi.org/10.1016/S1383-5742(00)00049-1

Andrews N, Miller E, Taylor B, Lingam R, Simmons A, Stowe J, Waight P. 2002. Recall bias, MMR, and autism. Arch Dis Child. 87(6):493-494. http://dx.doi.org/10.1136/adc.87.6.493

Anwar WA, Salama SI, el Serafy MM, Hemida SA, Hafez AS. 1994. Chromosomal aberrations and micronucleus frequency in nurses occupationally exposed to cytotoxic drugs. Mutagenesis. 9(4):315-317. http://dx.doi.org/10.1093/mutage/9.4.315

Axelsson G. 1990. Use of questionnaires in a study of spontaneous-abortion in a generalpopulation. J Epidemiol Commun H. 44(3):202-204. http://dx.doi.org/10.1136/jech.44.3.202

Benhamou S, Pot-Deprun J, Sancho-Garnier H, Chouroulinkov I. 1988. Sister chromatid exchanges and chromosomal aberrations in lymphocytes of nurses handling cytostatic agents. Int J Cancer. 41(3):350-353. http://dx.doi.org/10.1002/ijc.2910410305

Bernabeu-Martinez MA, Ramos Merino M, Santos Gago JM, Alvarez Sabucedo LM, WandenBerghe C, Sanz-Valero J. 2018. Guidelines for safe handling of hazardous drugs: A systematic review. PLoS One. 13(5):e0197172. http://dx.doi.org/10.1371/journal.pone.0197172

Berruyer M, Tanguay C, Caron NJ, Lefebvre M, Bussières JF. 2015. Multicenter study of environmental contamination with antineoplastic drugs in 36 Canadian Hospitals: A 2013 follow-up study. J Occup Environ Hyg. 12(2):87-94.

http://dx.doi.org/10.1080/15459624.2014.949725

Bigelow S, Schulz H, Dobish R, Chambers CR. 2009. Antineoplastic agent workplace contamination study: The Alberta Cancer Board Pharmacy perspective Phase III. J Oncol Pharm Pract. 15(3):157-160. http://dx.doi.org/10.1177/1078155208101097

Biró A, Fodor Z, Major J, Tompa A. 2011. Immunotoxicity monitoring of hospital staff occupationally exposed to cytostatic drugs. Pathol Oncol Res. 17(2):301-308.

http://dx.doi.org/10.1007/s12253-010-9317-z

Bolognesi C, Nucci MC, Colacci AM, Grilli S, Ippoliti F, Mucci N, Romano Spica V, Barbieri A, Canitano N, Chiozzotto D et al. 2005. [Biomonitoring of nurses occupationally exposed to antineoplastic drugs: The IMEPA Project]. Epidemiol Prev. 29(5-6 Suppl):91-95. 
Bonassi S, Znaor A, Ceppi M, Lando C, Chang WP, Holland N, Kirsch-Volders M, Zeiger E, Ban S, Barale R et al. 2007. An increased micronucleus frequency in peripheral blood lymphocytes predicts the risk of cancer in humans. Carcinogenesis. 28(3):625-631. http://dx.doi.org/10.1093/carcin/bgl177

Bonassi S, Znaor A, Norppa H, Hagmar L. 2004. Chromosomal aberrations and risk of cancer in humans: an epidemiologic perspective. Cytogenet Genome Res. 104(1-4):376-382. http://dx.doi.org/10.1159/000077519

Boughattas AB, Bouraoui S, Debbabi F, El Ghazel H, Saad A, Mrizak N. 2010. [Genotoxic risk assessment of nurses handling antineoplastic drugs]. Ann Biol Clin. 68(5):545-553. http://dx.doi.org/10.1684/abc.2010.0464

Bouraoui S, Brahem A, Tabka F, Mrizek N, Saad A, Elghezal H. 2011. Assessment of chromosomal aberrations, micronuclei and proliferation rate index in peripheral lymphocytes from Tunisian nurses handling cytotoxic drugs. Environ Toxicol Pharmacol. 31(1):250-257. http://dx.doi.org/10.1016/j.etap.2010.11.004

Bouyer J, Saurel-Cubizolles MJ, Grenier C, Aussel L, Job-Spira N. 1998. Ectopic pregnancy and occupational exposure of hospital personnel. Scand J Work Environ Health. 24(2):98-103. http://dx.doi.org/10.5271/sjweh.285

Buono-Michel M, Orsière T, Sari-Minodier I, Botta A. 2000. Cytogenetic biomonitoring of nurses handling cytostatics. Arch Mal Prof. 61(3):148-155.

Burgaz S, Karahalil B, Bayrak P, Taskin L, Yavuzaslan F, Bokesoy I, Anzion RB, Bos RP, Platin N. 1999. Urinary cyclophosphamide excretion and micronuclei frequencies in peripheral lymphocytes and in exfoliated buccal epithelial cells of nurses handling antineoplastics. Mutat Res. 439(1):97-104. http://dx.doi.org/10.1016/S1383-5718(98)00180-6

Burgaz S, Karahalil B, Canhi Z, Terzioglu F, Ancel G, Anzion RB, Bos RP, Huttner E. 2002. Assessment of genotoxic damage in nurses occupationally exposed to antineoplastics by the analysis of chromosomal aberrations. Hum Exp Toxicol. 21(3):129-135.

http://dx.doi.org/10.1191/0960327102ht230oa

Buschini A, Villarini M, Feretti D, Mussi F, Dominici L, Zerbini I, Moretti M, Ceretti E, Bonfiglioli R, Carrieri M et al. 2013. Multicentre study for the evaluation of mutagenic/carcinogenic risk in nurses exposed to antineoplastic drugs: Assessment of DNA damage. Occup Environ Med. 70(11):789-794. http://dx.doi.org/10.1136/oemed-2013-101475

Bussières JF, Tanguay C, Touzin K, Langlois E, Lefebvre M. 2012. Environmental contamination with hazardous drugs in Quebec hospitals. Can J Hosp Pharm. 65(6):428-435. http://dx.doi.org/10.4212/cjhp.v65i6.1190

Caciari T, Casale T, Tomei F, Samperi I, Tomei G, Capozzella A, Ripamonti K, Scala B, Andreozzi G, Nardone N et al. 2012. Exposure to antineoplastic drugs in health care and blood chemistry parameters. Clin Ter. 163(6):e387-392.

Canal-Raffin M, Khennoufa K, Martinez B, Goujon Y, Folch C, Ducint D, Titier K, Brochard P, Verdun-Esquer C, Molimard M. 2016. Highly sensitive LC-MS/MS methods for urinary 
biological monitoring of occupational exposure to cyclophosphamide, ifosfamide, and methotrexate antineoplastic drugs and routine application. J Chromatogr B. 1038:109-117. http://dx.doi.org/10.1016/j.jchromb.2016.10.021

Cardonick E, Usmani A, Ghaffar S. 2010. Perinatal outcomes of a pregnancy complicated by cancer, including neonatal follow-up after in utero exposure to chemotherapy: results of an international registry. Am J Clin Oncol. 33(3):221-228.

http://dx.doi.org/10.1097/COC.0b013e3181a44ca9

Cavallo D, Ursini CL, Omodeo-Sale E, Iavicoli S. 2007. Micronucleus induction and FISH analysis in buccal cells and lymphocytes of nurses administering antineoplastic drugs. Mutat Res. 628(1):11-18. http://dx.doi.org/10.1016/j.mrgentox.2006.10.014

Cavallo D, Ursini CL, Perniconi B, Francesco AD, Giglio M, Rubino FM, Marinaccio A, Iavicoli S. 2005. Evaluation of genotoxic effects induced by exposure to antineoplastic drugs in lymphocytes and exfoliated buccal cells of oncology nurses and pharmacy employees. Mutat Res. 587(1-2):45-51. http://dx.doi.org/10.1016/j.mrgentox.2005.07.008

Cavallo D, Ursini CL, Rondinone B, Iavicoli S. 2009. Evaluation of a suitable DNA damage biomarker for human biomonitoring of exposed workers. Environ Mol Mutagen. 50(9):781-790. http://dx.doi.org/10.1002/em.20501

Chu WC, Hon CY, Danyluk Q, Chua PP, Astrakianakis G. 2012. Pilot assessment of the antineoplastic drug contamination levels in British Columbian hospitals pre- and post-cleaning. J Oncol Pharm Pract. 18(1):46-51. http://dx.doi.org/10.1177/1078155211402106

Cogliano VJ, Baan R, Straif K, Grosse Y, Lauby-Secretan B, El Ghissassi F, Bouvard V, Benbrahim-Tallaa L, Guha N, Freeman C et al. 2011. Preventable exposures associated with human cancers. J Natl Cancer Inst. 103(24):1827-1839. http://dx.doi.org/10.1093/jnci/djr483

Colditz GA, Martin P, Stampfer MJ, Willett WC, Sampson L, Rosner B, Hennekens CH, Speizer FE. 1986. Validation of questionnaire information on risk-factors and disease outcomes in a prospective cohort study of women. Am J Epidemiol. 123(5):894-900.

http://dx.doi.org/10.1093/oxfordjournals.aje.a114319

Colditz GA, Stampfer MJ, Willett WC, Stason WB, Rosner B, Hennekens CH, Speizer FE. 1987. Reproducibility and validity of self-reported menopausal status in a prospective cohort study. Am J Epidemiol. 126(2):319-325. http://dx.doi.org/10.1093/aje/126.2.319

Collins A, Koppen G, Valdiglesias V, Dusinska M, Kruszewski M, Moller P, Rojas E, Dhawan A, Benzie I, Coskun E et al. 2014. The comet assay as a tool for human biomonitoring studies: the ComNet project. Mutat Res Rev Mutat Res. 759:27-39.

http://dx.doi.org/10.1016/j.mrrev.2013.10.001

Connor TH, Anderson RW, Sessink PJ, Broadfield L, Power LA. 1999. Surface contamination with antineoplastic agents in six cancer treatment centers in Canada and the United States. Am J Health Syst Pharm. 56(14):1427-1432.

Connor TH, DeBord DG, Pretty JR, Oliver MS, Roth TS, Lees PS, Krieg EF, Jr., Rogers B, Escalante CP, Toennis CA et al. 2010. Evaluation of antineoplastic drug exposure of health care 
workers at three university-based US cancer centers. J Occup Environ Med. 52(10):1019-1027. http://dx.doi.org/10.1097/JOM.0b013e3181f72b63

Connor TH, Lawson CC, Polovich M, McDiarmid MA. 2014. Reproductive health risks associated with occupational exposures to antineoplastic drugs in health care settings: a review of the evidence. J Occup Environ Med. 56(9):901-910.

http://dx.doi.org/10.1097/jom.0000000000000249

Connor TH, McDiarmid MA. 2006. Preventing occupational exposures to antineoplastic drugs in health care settings. CA Cancer J Clin. 56(6):354-365.

http://dx.doi.org/10.3322/canjclin.56.6.354

Cooke J, Williams J, Morgan RJ, Cooke P, Calvert RT. 1991. Use of cytogenetic methods to determine mutagenic changes in the blood of pharmacy personnel and nurses who handle cytotoxic agents. Am J Hosp Pharm. 48(6):1199-1205.

Cooney MA, Buck Louis GM, Sundaram R, McGuiness BM, Lynch CD. 2009. Validity of selfreported time to pregnancy. Epidemiology. 20(1):56-59.

http://dx.doi.org/10.1097/EDE.0b013e31818ef47e

Cornetta T, Pádua L, Testa A, Ievoli E, Festa F, Tranfo G, Baccelliere L, Cozzi R. 2008. Molecular biomonitoring of a population of nurses handling antineoplastic drugs. Mutat Res. 638(1-2):75-82. http://dx.doi.org/10.1016/j.mrfmmm.2007.08.017

Correa A, Cragan JD, Kucik JE, Alverson CJ, Gilboa SM, Balakrishnan R, Strickland MJ, Duke CW, O'Leary LA, Riehle-Colarusso T et al. 2007. Reporting birth defects surveillance data 19682003. Birth Defects Res A Clin Mol Teratol. 79(2):65-186. http://dx.doi.org/10.1002/bdra.20350

Couch J, dePerio MA. 2011. Health Hazard Evaluation Report: HETA-2010-0118-3142, September 2011. Chemotherapy drug evaluation at a medical laboratory - Pennsylvania. Govt Reports Announcements \& Index. (04):24.

Couch J, Gibbins J, Connor T. 2012. Health Hazard Evaluation Report: HETA-2010-0068-3156, April 2012. Chemotherapy drug evaluation at a veterinary teaching hospital - Michigan. Govt Reports Announcements \& Index. (15):32.

Couch J, Gibbins J, Connor TH. 2013. Evaluation of chemotherapy drug exposure at a veterinary teaching hospital in Michigan. J Occup Environ Hyg. 10(4):D45-51.

http://dx.doi.org/10.1080/15459624.2013.766561

Couch J, West C. 2012. Health Hazard Evaluation Report: HETA-2009-0148-3158, June 2012. Chemotherapy drug exposure at an oncology clinic - Florida. Govt Reports Announcements \& Index. (20):36.

Crauste-Manciet S, Sessink PJ, Ferrari S, Jomier JY, Brossard D. 2005. Environmental contamination with cytotoxic drugs in healthcare using positive air pressure isolators. Ann Occup Hyg. 49(7):619-628. http://dx.doi.org/10.1093/annhyg/mei045

Deng HP, Lou JL, Zhang MB, Wu W, Jin LF, Chen SJ, Zheng W, Wang BH, He JL. 2006. Detecting the cytogenetic effects in workers occupationally exposed to vincristine with four genetic tests. Mutat Res. 599(1-2):152-159. http://dx.doi.org/10.1016/j.mrfmmm.2006.02.003 
Deng HP, Zhang MB, He JL, Wu W, Jin LF, Zheng W, Lou JL, Wang BH. 2005. Investigating genetic damage in workers occupationally exposed to methotrexate using three genetic endpoints. Mutagenesis. 20(5):351-357. http://dx.doi.org/10.1093/mutage/gei048

Dranitsaris G, Johnston M, Poirier S, Schueller T, Milliken D, Green E, Zanke B. 2005. Are health care providers who work with cancer drugs at an increased risk for toxic events? A systematic review and meta-analysis of the literature. J Oncol Pharm Pract. 11(2):69-78. http://dx.doi.org/10.1191/1078155205jp155oa

El-Ebiary AA, Abuelfadl AA, Sarhan NI. 2013. Evaluation of genotoxicity induced by exposure to antineoplastic drugs in lymphocytes of oncology nurses and pharmacists. J Appl Toxicol. 33(3):196-201. http://dx.doi.org/10.1002/jat.1735

Elshamy K, El-Hadidi M, El-Roby M, Fouda M. 2010. Health hazards among oncology nurses exposed to chemotherapy drugs. Afr J Haematol Oncol. 1(3):70-78.

Ensslin AS, Huber R, Pethran A, Rommelt H, Schierl R, Kulka U, Fruhmann G. 1997. Biological monitoring of hospital pharmacy personnel occupationally exposed to cytostatic drugs: Urinary excretion and cytogenetics studies. Int Arch Occup Environ Health. 70(3):205208. http://dx.doi.org/10.1007/s004200050208

Ensslin AS, Pethran A, Schierl R, Fruhmann G. 1994a. Urinary platinum in hospital personnel occupationally exposed to platinum-containing antineoplastic drugs. Int Arch Occup Environ Health. 65(5):339-342. http://dx.doi.org/10.1007/BF00405699

Ensslin AS, Stoll Y, Pethran A, Pfaller A, Rommelt H, Fruhmann G. 1994b. Biological monitoring of cyclophosphamide and ifosfamide in urine of hospital personnel occupationally exposed to cytostatic drugs. Occup Environ Med. 51(4):229-233.

http://dx.doi.org/10.1136/oem.51.4.229

Evelo CT, Bos RP, Peters JG, Henderson PT. 1986. Urinary cyclophosphamide assay as a method for biological monitoring of occupational exposure to cyclophosphamide. Int Arch Occup Environ Health. 58(2):151-155. http://dx.doi.org/10.1007/BF00380766

Ferguson LR, Everts R, Robbie MA, Harvey V, Tempel D, Mak D, Gerred AJ. 1988. The use within New Zealand of cytogenetic approaches to monitoring of hospital pharmacists for exposure to cytotoxic drugs: report of a pilot study in Aukland. Aust J Hosp Pharm. 18(3):228233.

Fransman W, Huizer D, Tuerk J, Kromhout H. 2007a. Inhalation and dermal exposure to eight antineoplastic drugs in an industrial laundry facility. Int Arch Occup Environ Health. 80(5):396403. http://dx.doi.org/10.1007/s00420-006-0148-X

Fransman W, Kager H, Meijster T, Heederik D, Kromhout H, Portengen L, Blaauboer BJ. 2014. Leukemia from dermal exposure to cyclophosphamide among nurses in The Netherlands: quantitative assessment of the risk. Ann Occup Hyg. 58(3):271-282. http://dx.doi.org/10.1093/annhyg/met077 
Fransman W, Peelen S, Hilhorst S, Roeleveld N, Heederik D, Kromhout H. 2007b. A pooled analysis to study trends in exposure to antineoplastic drugs among nurses. Ann Occup Hyg. 51(3):231-239. http://dx.doi.org/10.1093/annhyg/mel081

Fransman W, Roeleveld N, Peelen S, de Kort W, Kromhout H, Heederik D. 2007c. Nurses with dermal exposure to antineoplastic drugs: reproductive outcomes. Epidemiology. 18(1):112-119. http://dx.doi.org/10.1097/01.ede.0000246827.44093.c1

Fucic A, Jazbec A, Mijic A, Seso-Simic D, Tomek R. 1998. Cytogenetic consequences after occupational exposure to antineoplastic drugs. Mutat Res. 416(1-2):59-66.

http://dx.doi.org/10.1016/S1383-5718(98)00084-9

Garaj-Vrhovac V, Kopjar N. 1998. Micronuclei in cytokinesis-blocked lymphocytes as an index of occupational exposure to antineoplastic drugs. Radiol Oncol. 32(4):385-392.

Goloni-Bertollo EM, Tajara EH, Manzato AJ, Varella-Garcia M. 1992. Sister chromatid exchanges and chromosome aberrations in lymphocytes of nurses handling antineoplastic drugs. Int J Cancer. 50(3):341-344. http://dx.doi.org/10.1002/ijc.2910500302

Grummt T, Grummt HJ, Schott G. 1993. Chromosomal aberrations in peripheral lymphocytes of nurses and physicians handling antineoplastic drugs. Mutat Res. 302(1):19-24.

http://dx.doi.org/10.1016/0165-7992(93)90085-A

Gudkov SV, Shilyagina NY, Vodeneev VA, Zvyagin AV. 2015. Targeted radionuclide therapy of human tumors. Int J Mol Sci. 17(1). http://dx.doi.org/10.3390/ijms17010033

Gunnarsdóttir H, Rafnsson V. 1995. Cancer incidence among Icelandic nurses. J Occup Environ Med. 37(3):307-312. http://dx.doi.org/10.1097/00043764-199503000-00007

Gunnarsdóttir HK, Aspelund T, Karlsson T, Rafnsson VV. 1997. Occupational risk factors for breast cancer among nurses. Int J Occup Environ Health. 3(4):254-258.

http://dx.doi.org/10.1179/oeh.1997.3.4.254

Guyatt G, Oxman AD, Akl EA, Kunz R, Vist G, Brozek J, Norris S, Falck-Ytter Y, Glasziou P, DeBeer $\mathrm{H}$ et al. 2011. GRADE guidelines: 1. Introduction-GRADE evidence profiles and summary of findings tables. J Clin Epidemiol. 64(4):383-394.

http://dx.doi.org/10.1016/j.jclinepi.2010.04.026

Hall AL, Davies HW, Demers PA, Nicol AM, Peters CE. 2013. Occupational exposures to antineoplastic drugs and ionizing radiation in Canadian veterinary settings: findings from a national surveillance project. Can J Public Health. 104(7):e460-465.

http://dx.doi.org/10.17269/cjph.104.4167

Halsen G, Kramer I. 2011. Assessing the risk to health care staff from long-term exposure to anticancer drugs--the case of monoclonal antibodies. J Oncol Pharm Pract. 17(1):68-80. http://dx.doi.org/10.1177/1078155210376847

Hama K, Fukushima K, Hirabatake M, Hashida T, Kataoka K. 2012. Verification of surface contamination of Japanese cyclophosphamide vials and an example of exposure by handling. $\mathrm{J}$ Oncol Pharm Pract. 18(2):201-206. http://dx.doi.org/10.1177/1078155211419543 
Hama T, Aoyama T, Shirai T, Higuchi S, Yokokawa T, Sugimoto Y. 2009. Environmental contamination due to preparation of cyclophosphamide and exposure of pharmacists to it. Jpn J Pharm Health Care Sci. 35(10):693-700. http://dx.doi.org/10.5649/jjphcs.35.693

Harris PE, Connor TH, Stevens KR, Theiss JC. 1992. Cytogenetic assessment of occupational exposure of nurses to antineoplastic agents. J Occup Med Toxicol. 1(3):243-254.

Hedmer M, Tinnerberg H, Axmon A, Jonsson BA. 2008. Environmental and biological monitoring of antineoplastic drugs in four workplaces in a Swedish hospital. Int Arch Occup Environ Health. 81(7):899-911. http://dx.doi.org/10.1007/s00420-007-0284-y

Hemminki K, Kyyronen P, Lindbohm ML. 1985. Spontaneous abortions and malformations in the offspring of nurses exposed to anaesthetic gases, cytostatic drugs, and other potential hazards in hospitals, based on registered information of outcome. J Epidemiol Commun H. 39(2):141147. http://dx.doi.org/10.1136/jech.39.2.141

Hessel H, Radon K, Pethran A, Maisch B, Grobmair S, Sautter I, Fruhmann G. 2001. The genotoxic risk of hospital, pharmacy and medical personnel occupationally exposed to cytostatic drugs--evaluation by the micronucleus assay. Mutat Res. 497(1-2):101-109.

http://dx.doi.org/10.1016/S1383-5718(01)00236-4

Hirst M, Tse S, Mills DG, Levin L, White DF. 1984. Occupational exposure to cyclophosphamide. Lancet. 323(8370):186-188. http://dx.doi.org/10.1016/S01406736(84)92111-1

Hola N, Sram RJ, Roznickova I. 1988. Evaluation of genotoxic risk in occupational exposure to cytostatics. Prac Lek. 40(4):154-157.

Hon CY, Teschke K, Chua P, Venners S, Nakashima L. 2011. Occupational exposure to antineoplastic drugs: Identification of job categories potentially exposed throughout the hospital medication system. Saf Health Work. 2(3):273-281.

http://dx.doi.org/10.5491/SHAW.2011.2.3.273

Hon CY, Teschke K, Demers PA, Venners S. 2014. Antineoplastic drug contamination on the hands of employees working troughout the hospital medication system. Ann Occup Hyg. 58(6):761-770. http://dx.doi.org/10.1093/annhyg/meu019

Hon CY, Teschke K, Shen H, Demers PA, Venners S. 2015. Antineoplastic drug contamination in the urine of Canadian healthcare workers. Int Arch Occup Environ Health. 88(7):933-941. http://dx.doi.org/10.1007/s00420-015-1026-1

International Agency for Research on Cancer (IARC). 1966. IARC Monograph on the Evaluation of the Carcinogenic Risk of Chemicals to Humans: Some Pharmaceutical Drugs. International Agency for Research on Cancer. Lyon, France: World Health Organization. p. 1514.

International Agency for Research on Cancer (IARC). 1980. IARC Monograph on the Evaluation of the Carcinogenic Risk of Chemical to Human: Some Antineoplastic and Immunosuppressive Agents. International Agency for Research on Cancer. Lyon, France: World Health Organization. p. 396. 
International Agency for Research on Cancer (IARC). 1981. IARC Monograph on the Evaluation of the Carcinogenic Risk of Chemicals to Humans: Some Antineoplastics \& Immunosuppressive Agents. International Agency for Research on Cancer. Lyon, France: World Health Organization. p. 1-411.

International Agency for Research on Cancer (IARC). 1990. IARC Monograph on the Evaluation of the Carcinogenic Risk of Chemicals to Humans: Pharmaceutical Drugs. International Agency for Research on Cancer. Lyon, France: World Health Organization. p. 1415.

International Agency for Research on Cancer (IARC). 2000. IARC Monograph on the Evaluation of the Carcinogenic Risk of Chemicals to Humans: Some Antineoplastics, Antivirals, and Other Pharmaceutical Drugs. International Agency for Research on Cancer. Lyon, France: World Health Organization. p. 1-531.

International Agency for Research on Cancer (IARC). 2012. A review of human carcinogens. Part A: Pharmaceuticals. IARC Working Group on the Evaluation of Carcinogenic Risks to Humans Meeting on 14-21 October 2008. Lyon, France: International Agency for Research on Cancer. http://monographs.iarc.fr/ENG/Monographs/vol100A/mono100A.pdf. [7 August 2017]

International Agency for Research on Cancer (IARC). 2016. IARC Monographs on the Evaluation of Carcinogenic Risks to Humans. Lyon, France: World Health Organization (WHO). http://monographs.iarc.fr/ENG/Classification/index.php. [Accessed: 10 July 2016]

Izdes S, Sardas S, Kadioglu E, Kaymak C, Ozcagli E. 2009. Assessment of genotoxic damage in nurses occupationally exposed to anaesthetic gases or antineoplastic drugs by the comet assay. J Occup Health. 51(3):283-286. http://dx.doi.org/10.1080/152873901300018011

Jakab MG, Major J, Tompa A. 2001. Follow-up genotoxicological monitoring of nurses handling antineoplastic drugs. J Toxicol Environ Health A. 62(5):307-318.

http://dx.doi.org/10.1080/152873901300018011

Janes A, Tanguay C, Caron NJ, Bussieres JF. 2015. Environmental contamination with cyclophosphamide, ifosfamide, and methotrexate: A study of 51 Canadian centres. Can J Hosp Pharm. 68(4):279-289.

Janssens T, Brouwers EE, de Vos JP, de Vries N, Schellens JH, Beijnen JH. 2015a. Determination of platinum surface contamination in veterinary and human oncology centres using inductively coupled plasma mass spectrometry. Vet Comp Oncol. 13(3):305-313. http://dx.doi.org/10.1111/vco.12049

Janssens T, Brouwers EE, de Vos JP, de Vries N, Schellens JH, Beijnen JH. 2015b. Inductively coupled plasma mass-spectrometric determination of platinum in excretion products of clientowned pet dogs. Vet Comp Oncol. 13(2):124-132. http://dx.doi.org/10.1111/vco.12025

Jeebhay M, Mbuli S, Uebel R. 1993. Assessment of exposure to chloramphenicol and azathioprine among workers in a South African pharmaceutical plant. Int Arch Occup Environ Health. 65(S1):S119-122. http://dx.doi.org/10.1007/BF00381321 
Jochimsen PR, Corder MP, Lachenbruch PA, Spaight ME. 1988. Preparation and administration of chemotherapy. Haematological consequences for hospital-based nurses. Med Toxicol Adverse Drug Exp. 3(1):59-63. http://dx.doi.org/10.1007/BF03259931

Joffe M, Villard L, Li ZM, Plowman R, Vessey M. 1993. Long-term recall of time-to-pregnancy. Fertil Steril. 60(1):99-104. http://dx.doi.org/10.1016/S0015-0282(16)56044-0

Karahalil B, Ilter Akkoyunlu K. 2004. Determination of urinary cyclophosphamide in oncology nurses handling antineoplastic drugs by gas chromatography-mass spectrometry. FABAD J Pharm Sci. 28(3):125-130.

Kašuba V, Rozgaj R, Garaj-Vrhovac V. 1999. Analysis of sister chromatid exchange and micronuclei in peripheral blood lymphocytes of nurses handling cytostatic drugs. J Appl Toxicol. 19(6):401-404. http://dx.doi.org/10.1002/(SICI)1099-1263(199911/12)19:6<401::AIDJAT592>3.0.CO;2-H

Kevekordes S, Gebel TW, Hellwig M, Dames W, Dunkelberg H. 1998. Human effect monitoring in cases of occupational exposure to antineoplastic drugs: A method comparison. Occup Environ Med. 55(3):145-149. http://dx.doi.org/10.1136/oem.55.3.145

Kibby T. 2017. A review of surface wipe sampling compared to biologic monitoring for occupational exposure to antineoplastic drugs. J Occup Environ Hyg. 14(3):159-174. http://dx.doi.org/10.1080/15459624.2016.1237026

Kiffmeyer TK, Kube C, Opiolka S, Schmidt KG, Schöppe G, Sessink PJM. 2002. Vapour pressures, evaporation behaviour and airborne concentrations of hazardous drugs: Implications for occupational safety. Pharm J. 268(7188):331-337.

Kiffmeyer TK, Tuerk J, Hahn M, Stuetzer H, Hadtstein C, Heinemann A, Eickmann U. 2013. Application and assessment of a regular environmental monitoring of the antineoplastic drug contamination level in pharmacies - the MEWIP project. Ann Occup Hyg. 57(4):444-455. http://dx.doi.org/10.1093/annhyg/mes081

Kirsch-Volders M, Plas G, Elhajouji A, Lukamowicz M, Gonzalez L, Vande Loock K, Decordier I. 2011. The in vitro MN assay in 2011: origin and fate, biological significance, protocols, high throughput methodologies and toxicological relevance. Arch Toxicol. 85(8):873-899. http://dx.doi.org/10.1007/s00204-011-0691-4

Klahn S. 2014. Chemotherapy safety in clinical veterinary oncology. Vet Clin North Am Small Anim Pract. 44(5):941-963. http://dx.doi.org/10.1016/j.cvsm.2014.05.009

Knobloch A, Mohring SA, Eberle N, Nolte I, Hamscher G, Simon D. 2010. Drug residues in serum of dogs receiving anticancer chemotherapy. J Vet Intern Med. 24(2):379-383. http://dx.doi.org/10.1111/j.1939-1676.2009.0469.x

Konate A, Poupon J, Villa A, Garnier R, Hasni-Pichard H, Mezzaroba D, Fernandez G, Pocard M. 2011. Evaluation of environmental contamination by platinum and exposure risks for healthcare workers during a heated intraperitoneal perioperative chemotherapy (HIPEC) procedure. J Surg Oncol. 103(1):6-9. http://dx.doi.org/10.1002/jso.21740 
Kopjar N, Garaj-Vrhovac V. 2001. Application of the alkaline comet assay in human biomonitoring for genotoxicity: A study on Croatian medical personnel handling antineoplastic drugs. Mutagenesis. 16(1):71-78. http://dx.doi.org/10.1093/mutage/16.1.71

Kopjar N, Garaj-Vrhovac V, Kasuba V, Rozgaj R, Ramic S, Pavlica V, Zeljezic D. 2009. Assessment of genotoxic risks in Croatian health care workers occupationally exposed to cytotoxic drugs: A multi-biomarker approach. Int J Hyg Environ Health. 212(4):414-431. http://dx.doi.org/10.1016/j.ijheh.2008.10.001

Kopp B, Crauste-Manciet S, Guibert A, Mourier W, Guerrault-Moro MN, Ferrari S, Jomier JY, Brossard D, Schierl R. 2013a. Environmental and biological monitoring of platinum-containing drugs in two hospital pharmacies using positive air pressure isolators. Ann Occup Hyg. 57(3):374-383. http://dx.doi.org/10.1093/annhyg/mes073

Kopp B, Schierl R, Nowak D. 2013b. Evaluation of working practices and surface contamination with antineoplastic drugs in outpatient oncology health care settings. Int Arch Occup Environ Health. 86(1):47-55. http://dx.doi.org/10.1007/s00420-012-0742-z

Korczowska E, Jankowiak-Gracz H. 2013. How can we improve safe handling of antineoplastic drugs: Can devices be helpful. Eur J Onc Pharm. 7(1):5-7.

Krepinsky A, Bryant DW, Davison L, Young B, Heddle J, McCalla DR, Douglas G, Michalko K. 1990. Comparison of three assays for genetic effects of antineoplastic drugs on cancer patients and their nurses. Environ Mol Mutagen. 15(2):83-92.

http://dx.doi.org/10.1002/em.2850150205

Krstev S, Perunicic B, Vidakovic A. 2003. Work practice and some adverse health effects in nurses handling antineoplastic drugs. Med Lav. 94(5):432-439.

Ladeira C, Viegas S, Pádua M, Gomes M, Carolino E, Gomes MC, Brito M. 2014. Assessment of genotoxic effects in nurses handling cytostatic drugs. J Toxicol Environ Health A. 77(1416):879-887. http://dx.doi.org/10.1080/15287394.2014.910158

Laffon B, Teixeira JP, Silva S, Loureiro J, Torres J, Pasaro E, Mendez J, Mayan O. 2005. Genotoxic effects in a population of nurses handling antineoplastic drugs, and relationship with genetic polymorphisms in DNA repair enzymes. Am J Ind Med. 48(2):128-136.

http://dx.doi.org/10.1080/15287394.2014.910158

Lawson CC, Rocheleau CM, Whelan EA, Lividoti Hibert EN, Grajewski B, Spiegelman D, Rich-Edwards JW. 2012. Occupational exposures among nurses and risk of spontaneous abortion. Am J Obstet Gynecol. 206(4):327.e321-327.e328.

http://dx.doi.org/10.1016/j.ajog.2011.12.030

Lawson CC, Whelan EA, Hibert EN, Grajewski B, Spiegelman D, Rich-Edwards JW. 2009. Occupational factors and risk of preterm birth in nurses. Am J Obstet Gynecol. 200(1):51 e5158. http://dx.doi.org/10.1016/j.ajog.2008.08.006

Li ML, Zhang YY, Wang QX, Pei Y, Xiao CS. 2005. [Effects of antineoplastics drug on Tlymphocytes subsets in nurses of occupational exposure to antineoplastic drugs]. Zhonghua Lao Dong Wei Sheng Zhi Ye Bing Za Zhi. 23(6):450-451. 
Lie J, Andersen A, Kjaerheim K. 2007. Cancer risk among 43000 Norwegian nurses. Scand J Work Environ Health. 33(1):66-73. http://dx.doi.org/doi:10.5271/sjweh.1066

Lorente C, Cordier S, Bergeret A, De Walle HE, Goujard J, Ayme S, Knill-Jones R, Calzolari E, Bianchi F. 2000. Maternal occupational risk factors for oral clefts. Occupational Exposure and Congenital Malformation Working Group. Scand J Work Environ Health. 26(2):137-145. http://dx.doi.org/10.5271/sjweh.523

Machado-Santelli GM, Cerqueira EM, Oliveira CT, Pereira CA. 1994. Biomonitoring of nurses handling antineoplastic drugs. Mutat Res. 322(3):203-208. http://dx.doi.org/10.1016/0165$\underline{1218(94) 90007-8}$

Mader RM, Kokalj A, Kratochvil E, Pilger A, Rudiger HW. 2009. Longitudinal biomonitoring of nurses handling antineoplastic drugs. J Clin Nurs. 18(2):263-269.

http://dx.doi.org/10.1111/j.1365-2702.2007.02189.x

Maeda S, Miyawaki K, Matsumoto S, Oishi M, Miwa Y, Kurokawa N. 2010. Evaluation of environmental contaminations and occupational exposures involved in preparation of chemotherapeutic drugs. Yakugaku Zasshi. 130(6):903-910.

http://dx.doi.org/10.1248/yakushi.130.903

Mahmoodi M, Soleyman-Jahi S, Zendehdel K, Mozdarani H, Azimi C, Farzanfar F, Safari Z, Mohagheghi MA, Khaleghian M, Divsalar K et al. 2017. Chromosomal aberrations, sister chromatid exchanges, and micronuclei in lymphocytes of oncology department personnel handling anti-neoplastic drugs. Drug Chem Toxicol. 40(2):235-240.

http://dx.doi.org/10.1080/01480545.2016.1209678

Mahrous HS, Ismail SR, Hashishe MM, Kohail HM. 1998. Sister chromatid exchanges and chromosome aberrations in lymphocytes of medical personnel handling cytostatic drugs. J Egypt Public Health Assoc. 73(3-4):297-323.

Maluf SW, Erdtmann B. 2000a. Evaluation of occupational genotoxic risk in a Brazilian hospital. Genet Mol Biol. 23(2):485-488. http://dx.doi.org/10.1590/S1415-47572000000200040

Maluf SW, Erdtmann B. 2000b. Follow-up study of the genetic damage in lymphocytes of pharmacists and nurses handling antineoplastic drugs evaluated by cytokinesis-block micronuclei analysis and single cell gel electrophoresis assay. Mutat Res. 471(1-2):21-27.

http://dx.doi.org/10.1016/S1383-5718(00)00107-8

Martins I, Apostoli P, Della Rosa HV. 2008. Cyclophosphamide levels in sites of preparation and administration of antineoplastic drugs. Lat Am J Pharm. 27(2):217-223.

Mateuca R, Lombaert N, Aka PV, Decordier I, Kirsch-Volders M. 2006. Chromosomal changes: induction, detection methods and applicability in human biomonitoring. Biochimie. 88(11):15151531. http://dx.doi.org/10.1016/j.biochi.2006.07.004

McAbee RR, Gallucci BJ, Checkoway H. 1993. Adverse reproductive outcomes and occupational exposures among nurses: An investigation of multiple hazardous exposures. AAOHN J. 41(3):110-119. 
McDevitt JJ, Lees PS, McDiarmid MA. 1993. Exposure of hospital pharmacists and nurses to antineoplastic agents. J Occup Med. 35(1):57-60.

McDiarmid MA, Kolodner K, Humphrey F, Putman D, Jacobson-Kram D. 1992. Baseline and phosphoramide mustard-induced sister-chromatid exchanges in pharmacists handling anti-cancer drugs. Mutat Res. 279(3):199-204.

McDiarmid MA, Oliver MS, Roth TS, Rogers B, Escalante C. 2010. Chromosome 5 and 7 abnormalities in oncology personnel handling anticancer drugs. J Occup Environ Med. 52(10):1028-1034. http://dx.doi.org/10.1097/JOM.0b013e3181f73ae6

McDiarmid MA, Rogers B, Oliver MS. 2014. Chromosomal effects of non-alkylating drug exposure in oncology personnel. Environ Mol Mutagen. 55(4):369-374.

http://dx.doi.org/10.1002/em.21852

McDonald AD, McDonald JC, Armstrong B, Cherry NM, Cote R, Lavoie J, Nolin AD, Robert D. 1988a. Congenital defects and work in pregnancy. Brit J Ind Med. 45(9):581-588.

McDonald AD, McDonald JC, Armstrong B, Cherry NM, Cote R, Lavoie J, Nolin AD, Robert D. 1988b. Fetal death and work in pregnancy. Brit J Ind Med. 45(3):148-157.

Medková J. 1990. Cytogenetic analysis of peripheral lymphocytes in occupationally exposed health personnel. Acta Univ Palacki Olomuc Fac Med. 126:93-106.

Medková J. 1991a. An analysis of the health condition of personnel exposed to cytostatics at an oncology unit. Acta Univ Palacki Olomuc Fac Med. 130:311-322.

Medková J. 1991b. Analysis of the health condition of the children born to the personnel exposed to cytostatics at an oncology unit. Acta Univ Palacki Olomuc Fac Med. 130:323-332.

Meijster T, Fransman W, Veldhof R, Kromhout H. 2006. Exposure to antineoplastic drugs outside the hospital environment. Ann Occup Hyg. 50(7):657-664.

http://dx.doi.org/10.1093/annhyg/mel023

Merger D, Tanguay C, Langlois E, Lefebvre M, Bussieres JF. 2014. Multicenter study of environmental contamination with antineoplastic drugs in 33 Canadian hospitals. Int Arch Occup Environ Health. 87(3):307-313. http://dx.doi.org/10.1007/s00420-013-0862-0

Milković-Kraus S, Horvat D. 1991. Chromosomal abnormalities among nurses occupationally exposed to antineoplastic drugs. Am J Ind Med. 19(6):771-774.

http://dx.doi.org/10.1002/ajim.4700190609

Minoia C, Turci R, Sottani C, Schiavi A, Perbellini L, Angeleri S, Draicchio F, Apostoli P. 1998. Application of high performance liquid chromatography/tandem mass spectrometry in the environmental and biological monitoring of health care personnel occupationally exposed to cyclophosphamide and ifosfamide. Rapid Commun Mass Spectrom. 12(20):1485-1493. http://dx.doi.org/10.1002/(SICI)1097-0231(19981030)12:20<1485::AID-RCM333>3.0.CO;2-N

Mistry AR, Felix CA, Whitmarsh RJ, Mason A, Reiter A, Cassinat B, Parry A, Walz C, Wiemels JL, Segal MR et al. 2005. DNA topoisomerase II in therapy-related acute promyelocytic leukemia. N Engl J Med. 352(15):1529-1538. http://dx.doi.org/10.1056/NEJMoa042715 
Miyake T, Iwamoto T, Tanimura M, Okuda M. 2013. Impact of closed-system drug transfer device on exposure of environment and healthcare provider to cyclophosphamide in Japanese hospital. Springerplus. 2(1):273. http://dx.doi.org/10.1186/2193-1801-2-273

Moretti M, Grollino MG, Pavanello S, Bonfiglioli R, Villarini M, Appolloni M, Carrieri M, Sabatini L, Dominici L, Stronati L et al. 2015. Micronuclei and chromosome aberrations in subjects occupationally exposed to antineoplastic drugs: A multicentric approach. Int Arch Occup Environ Health. 88(6):683-695. http://dx.doi.org/10.1007/s00420-014-0993-y

Moretti M, Villarini M, Dominici L, Fatigoni C, Dell'omo M, Elisei E, Muzi G, Monarca S. 2013. [Evaluation of genotoxic effects in subjects occupationally exposed to antineoplastic drugs]. Ig Sanita Pubbl. 69(1):55-77.

Morton LM, Dores GM, Tucker MA, Kim CJ, Onel K, Gilbert ES, Fraumeni Jr JF, Curtis RE. 2013. Evolving risk of therapy-related acute myeloid leukemia following cancer chemotherapy among adults in the United States, 1975-2008. Blood. 121(15):2996-3004. http://dx.doi.org/10.1182/blood-2012-08-448068

Mrđanović J, Jungic S, Solajic S, Bogdanovic V, Jurisic V. 2012. Effects of orally administered antioxidants on micronuclei and sister chromatid exchange frequency in workers professionally exposed to antineoplastic agents. Food Chem Toxicol. 50(8):2937-2944.

http://dx.doi.org/10.1016/j.fct.2012.04.027

Mušák L, Halasova E, Matakova T, Letkova L, Vodickova L, Buchancova J, Hudeckova H, Osina O, Soucek P, Vodicka P. 2009a. Comparison of chromosomal aberrations frequency and polymorphism of GSTs genes in workers occupationally exposed to cytostatics or anaesthetics. Interdiscip Toxicol. 2(3):190-194. http://dx.doi.org/10.2478/v10102-009-0016-0

Mušák L, Polakova V, Halasova E, Osina O, Vodickova L, Buchancova J, Hudeckova H, Vodicka P. 2009b. Effect of occupational exposure to cytostatics and nucleotide excision repair polymorphism on chromosomal aberrations frequency. Interdiscip Toxicol. 2(1):13-17. http://dx.doi.org/10.2478/v10102-009-0002-6

Mušák L, Smerhovsky Z, Halasova E, Osina O, Letkova L, Vodickova L, Polakova V, Buchancova J, Hemminki K, Vodicka P. 2013. Chromosomal damage among medical staff occupationally exposed to volatile anesthetics, antineoplastic drugs, and formaldehyde. Scand J Work Environ Health. 39(6):618-630. http://dx.doi.org/10.5271/sjweh.3358

Mušák L, Vodicka P, Klimentova G, Soucek P, Hanova M, Mikulkova R, Buchancova J, Vodickova L, Polakova V, Pec M. 2006. Chromosomal damage and polymorphisms of DNA repair genes XRCC1 and XRCC3 in workers exposed to cytostatics. Neuro Endocrinol Lett. 27 Suppl 2:57-60.

National Toxicology Program (NTP). 2008. NTP-CERHR Monograph on the Potential Human Reproductive and Developmental Effects of Hydroxyurea. National Toxicology Program Center for Evaluation of Risks to Human Reproduction. Research Triangle Park, NC: US Department of Health and Human Services, Public Health Service. p. vii-viii, v, ix-III1.

National Toxicology Program (NTP). 2013. NTP Monograph: Developmental Effects and Pregnancy Outcomes Associated With Cancer Chemotherapy Use During Pregnancy. National 
Toxicology Program. 2014/04/17 ed. Research Triangle Park, NC: US Department of Health and Human Services, Public Health Service. p. i-214.

National Toxicology Program (NTP). 2015a. Health Assessment Workspace Collaborative (HAWC) page for occupational chemotherapy. https://hawcproject.org/assessment/196/.

National Toxicology Program (NTP). 2015b. MGRAPH-5: Occupational exposure to cancer chemotherapy agents supplementary files. Research Triangle Park, NC: U.S. Department of Health and Human Services, National Institute of Environmental Health Sciences, National Toxicology Program. https://doi.org/10.22427/NTP-DATA-MONOGRAPH5.

National Toxicology Program (NTP). 2016. Report on Carcinogens, 14th Edition. Research Triangle Park, NC: US Department of Health and Human Services, Public Health Service. https://ntp.niehs.nih.gov/go/roc14 [7 August 2017]

Nieuwenhuijsen M. 2003. Exposure Assessment in Occupational and Environmental Epidemiology. New York, New York: Oxford University Press, Inc.

Nikula E, Kiviniitty K, Leisti J, Taskinen PJ. 1984. Chromosome aberrations in lymphocytes of nurses handling cytostatic agents. Scand J Work Environ Health. 10(2):71-74.

http://dx.doi.org/10.5271/sjweh.2355

Norppa H, Bonassi S, Hansteen IL, Hagmar L, Stromberg U, Rossner P, Boffetta P, Lindholm C, Gundy S, Lazutka J et al. 2006. Chromosomal aberrations and SCEs as biomarkers of cancer risk. Mutat Res. 600(1-2):37-45. http://dx.doi.org/10.1016/j.mrfmmm.2006.05.030

Nyman HA, Jorgenson JA, Slawson MH. 2007. Workplace contamination with antineoplastic agents in a new cancer hospital using a closed-system drug transfer device. Hosp Pharm. 42(3):219-225. http://dx.doi.org/10.1310/hpj4203-219

Occupational Safety and Health Administration (OSHA). 1986. Work practice guidelines for personnel dealing with cytotoxic (antineoplastic) drugs. OSHA Publication 8-1.1. Washington, DC: US Department of Labor, Occupational Safety and Health Administration.

https://www.osha.gov/pls/oshaweb/owadisp.show_document?p_table=DIRECTIVES\&p_id=170 2. [June 1, 2016]

Occupational Safety and Health Administration (OSHA). 1999. Technical Manual (OTM), Section VI: Chapter 2 Controlling Occupational Exposure to Hazardous Drugs. Directive number: TED 01-00-015 [TED 1-0.15A]. Washington, DC: United States Department of Labor, Occupational Safety and Health Administration.

http://www.osha.gov/dts/osta/otm/otm_vi/otm_vi_2.html\#2.

Odio AD, Duharte AB, Carnesoltas D, Ibrahín L, García R, Loaces EL, Almarales DC, Mora YL, Cabrera LG. 2004. Efectos citogenéticos por exposición ocupacional a citostáticos. Revista Médica de Instituto Mexicano del Seguro Social. 42(6):487-492.

Odraska P, Dolezalova L, Kuta J, Oravec M, Piler P, Synek S, Blaha L. 2014. Association of surface contamination by antineoplastic drugs with different working conditions in hospital pharmacies. Arch Environ Occup Health. 69(3):148-158.

http://dx.doi.org/10.1080/19338244.2013.763757 
Odraska P, Dolezalova L, Piler P, Oravec M, Blaha L. 2011. Utilization of the solid sorbent media in monitoring of airborne cyclophosphamide concentrations and the implications for occupational hygiene. J Environ Monit. 13(5):1480-1487. http://dx.doi.org/10.1039/c0em00660b

Oestreicher U, Stephan G, Glatzel M. 1990. Chromosome and SCE analysis in peripheral lymphocytes of persons occupationally exposed to cytostatic drugs handled with and without use of safety covers. Mutat Res. 242(4):271-277. http://dx.doi.org/10.1016/0165-1218(90)90045-4

Organization for Economic Cooperation and Development (OECD). 2016a. Test No. 473: In Vitro Mammalian Chromosomal Aberration Test. Paris, France: OECD Publishing. http://dx.doi.org/10.1787/9789264264649-en [2 October 2017]

Organization for Economic Cooperation and Development (OECD). 2016b. Test No. 487: In Vitro Mammalian Cell Micronucleus Test. Paris, France: OECD Publishing. http://dx.doi.org/10.1787/9789264224438-en. [2 October 2017]

Organization for Economic Cooperation and Development (OECD). 2016c. Test No. 489: In Vivo Mammalian Alkaline Comet Assay. Paris, France: OECD Publishing. http://dx.doi.org/10.1787/9789264264885-en [2 October 2017]

Pedersen-Bjergaard J. 2005. Insights into leukemogenesis from therapy-related leukemia. N Engl J Med. 352(15):1591-1594. http://dx.doi.org/10.1056/NEJMe048336

Pedersen-Bjergaard J, Andersen MK, Christiansen DH, Nerlov C. 2002. Genetic pathways in therapy-related myelodysplasia and acute myeloid leukemia. Blood. 99(6):1909-1912. http://dx.doi.org/10.1182/blood.V99.6.1909

Peelen S, Roeleveld N, Heederik D, Kromhout H, De Kort W. 1999. Reproductietoxische effecten bij ziekenhuispersoneel [Toxic effects on reproduction in hospital personnel]. The Hague, Netherlands: Dutch Ministry of Social Affairs and Employment.

Pethran A, Schierl R, Hauff K, Grimm CH, Boos KS, Nowak D. 2003. Uptake of antineoplastic agents in pharmacy and hospital personnel. Part I: Monitoring of urinary concentrations. Int Arch Occup Environ Health. 76(1):5-10. http://dx.doi.org/10.1007/s00420-002-0383-8

Pilger A, Kohler I, Stettner H, Mader RM, Rizovski B, Terkola R, Diem E, Franz-Hainzl E, Konnaris C, Valic E et al. 2000. Long-term monitoring of sister chromatid exchanges and micronucleus frequencies in pharmacy personnel occupationally exposed to cytostatic drugs. Int Arch Occup Environ Health. 73(7):442-448. http://dx.doi.org/10.1007/s004200000164

Pohlová H, Cerna M, Rossner P. 1986. Chromosomal aberrations, SCE and urine mutagenicity in workers occupationally exposed to cytostatic drugs. Mutat Res. 174(3):213-217. http://dx.doi.org/10.1016/0165-7992(86)90154-5

Poupeau C, Tanguay C, Plante C, Gagne S, Caron N, Bussieres JF. 2017. Pilot study of biological monitoring of four antineoplastic drugs among Canadian healthcare workers. J Oncol Pharm Pract. 23(5):323-332. http://dx.doi.org/10.1177/1078155216643860

Quansah R, Jaakkola JJ. 2010. Occupational exposures and adverse pregnancy outcomes among nurses: a systematic review and meta-analysis. J Womens Health. 19(10):1851-1862.

http://dx.doi.org/10.1089/jwh.2009.1876 
Ramphal R, Bains T, Goulet G, Vaillancourt R. 2015. Occupational exposure to chemotherapy of pharmacy personnel at a single centre. Can J Hosp Pharm. 68(2):104-112.

Ramphal R, Bains T, Vaillancourt R, Osmond MH, Barrowman N. 2014. Occupational exposure to cyclophosphamide in nurses at a single center. J Occup Environ Med. 56(3):304-312. http://dx.doi.org/10.1097/JOM.0000000000000097

Ratner PA, Spinelli JJ, Beking K, Lorenzi M, Chow Y, Teschke K, Le ND, Gallagher RP, Dimich-Ward H. 2010. Cancer incidence and adverse pregnancy outcome in registered nurses potentially exposed to antineoplastic drugs. BMC Nurs. 9:15. http://dx.doi.org/10.1186/1472$\underline{6955-9-15}$

Rekhadevi PV, Sailaja N, Chandrasekhar M, Mahboob M, Rahman MF, Grover P. 2007. Genotoxicity assessment in oncology nurses handling anti-neoplastic drugs. Mutagenesis. 22(6):395-401. http://dx.doi.org/10.1093/mutage/gem032

Rodríguez-Montero HM, Argote-Pelegrino E, Díaz-Curbelo A, Cuétara-Lugo EB. 2016. Genotoxicity biomarkers for monitoring occupational exposure to antineoplastic drugs. J Pharm Pharmacogn Res. 4(3):122-133.

Rogers B, Emmett EA. 1987. Handling antineoplastic agents: Urine mutagenicity in nurses. Image J Nurs Sch. 19(3):108-113. http://dx.doi.org/10.1111/j.1547-5069.1987.tb00604.x

Rombaldi F, Cassini C, Salvador M, Saffi J, Erdtmann B. 2009. Occupational risk assessment of genotoxicity and oxidative stress in workers handling anti-neoplastic drugs during a working week. Mutagenesis. 24(2):143-148. http://dx.doi.org/10.1093/mutage/gen060

Rooney AA, Boyles AL, Wolfe MS, Bucher JR, Thayer KA. 2014. Systematic review and evidence integration for literature-based environmental health science assessments. Environ Health Perspect. 122(7):711-718. http://dx.doi.org/10.1289/ehp.1307972

Rooney AA, Cooper GS, Jahnke GD, Lam J, Morgan RL, Boyles AL, Ratcliffe JM, Kraft AD, Schunemann HJ, Schwingl P et al. 2016. How credible are the study results? Evaluating and applying internal validity tools to literature-based assessments of environmental health hazards. Environ Int. 92-93:617-629. https://doi.org/10.1016/j.envint.2016.01.005

Roth S, Norppa H, Jarventaus H, Kyyronen P, Ahonen M, Lehtomaki J, Sainio H, Sorsa M. 1994. Analysis of chromosomal aberrations, sister-chromatid exchanges and micronuclei in peripheral lymphocytes of pharmacists before and after working with cytostatic drugs. Mutat Res. 325(4):157-162. http://dx.doi.org/10.1016/0165-7992(94)90079-5

Roussel C, Witt KL, Shaw PB, Connor TH. 2017. Meta-analysis of chromosomal aberrations as a biomarker of exposure in healthcare workers occupationally exposed to antineoplastic drugs.

Mutat Res. 770:35-45. http://dx.doi.org/10.1016/j.mrrev.2017.08.002

Rubeš J, Kucharova S, Vozdova M, Musilova P, Zudova Z. 1998. Cytogenetic analysis of peripheral lymphocytes in medical personnel by means of FISH. Mutat Res. 412(3):293-298. http://dx.doi.org/10.1016/S1383-5718(97)00201-5

Sabatini L, Barbieri A, Lodi V, Violante FS. 2012. Biological monitoring of occupational exposure to antineoplastic drugs in hospital settings. Med Lav. 103(5):394-401. 
Sarto F, Trevisan A, Tomanin R, Canova A, Fiorentino M. 1990. Chromosomal aberrations, sister chromatid exchanges, and urinary thioethers in nurses handling antineoplastic drugs. Am J Ind Med. 18(6):689-695. http://dx.doi.org/10.1002/ajim.4700180607

Sasaki M, Dakeishi M, Hoshi S, Ishii N, Murata K. 2008. Assessment of DNA damage in Japanese nurses handling antineoplastic drugs by the comet assay. J Occup Health. 50(1):7-12. http://dx.doi.org/10.1539/joh.50.7

Saurel-Cubizolles MJ, Job-Spira N, Estryn-Behar M. 1993. Ectopic pregnancy and occupational exposure to antineoplastic drugs. Lancet. 341(8854):1169-1171. http://dx.doi.org/10.1016/0140$\underline{6736(93) 91000-C}$

Schaumburg I, Olsen J. 1989. Time to pregnancy among Danish pharmacy assistants. Scand J Work Environ Health. 15:222-226. http://dx.doi.org/10.5271/sjweh.1859

Schaumburg I, Olsen J. 1990a. Congenital malformations and death among the offspring of Danish pharmacy assistants. Am J Ind Med. 18(5):555-564. http://dx.doi.org/10.1002/ajim.4700180506

Schaumburg I, Olsen J. 1990b. Risk of spontaneous abortion among Danish pharmacy assistants. Scand J Work Environ Health. 16(3):169-174. http://dx.doi.org/10.5271/sjweh.1797

Schaumburg I, Olsen J. 1991. Birth weight and gestational age among children of Danish pharmacy assistants. J Epidemiol Commun H. 45(1):49-51.

http://dx.doi.org/10.1136/jech.45.1.49

Schierl R, Masini C, Groeneveld S, Fischer E, Bohlandt A, Rosini V, Paolucci D. 2016.

Environmental contamination by cyclophosphamide preparation: Comparison of conventional manual production in biological safety cabinet and robot-assisted production by APOTECAchemo. J Oncol Pharm Pract. 22(1):37-45. http://dx.doi.org/10.1177/1078155214551316

Schmaus G, Schierl R, Funck S. 2002. Monitoring surface contamination by antineoplastic drugs using gas chromatography-mass spectrometry and voltammetry. Am J Health Syst Pharm. 59(10):956-961.

Schreiber C, Radon K, Pethran A, Schierl R, Hauff K, Grimm CH, Boos KS, Nowak D. 2003. Uptake of antineoplastic agents in pharmacy personnel. Part II: Study of work-related risk factors. Int Arch Occup Environ Health. 76(1):11-16. http://dx.doi.org/10.1007/s00420-002$\underline{0385-6}$

Selevan SG, Lindbohm ML, Hornung RW, Hemminki K. 1985. A study of occupational exposure to antineoplastic drugs and fetal loss in nurses. N Engl J Med. 313(19):1173-1178. http://dx.doi.org/10.1056/NEJM198511073131901

Sessink PJ, Anzion RB, Van den Broek PH, Bos RP. 1992a. Detection of contamination with antineoplastic agents in a hospital pharmacy department. Pharm Weekbl Sci. 14(1):16-22.

Sessink PJ, Boer KA, Scheefhals AP, Anzion RB, Bos RP. 1992b. Occupational exposure to antineoplastic agents at several departments in a hospital. Environmental contamination and 
excretion of cyclophosphamide and ifosfamide in urine of exposed workers. Int Arch Occup Environ Health. 64(2):105-112. http://dx.doi.org/10.1007/BF00381477

Sessink PJ, Cerna M, Rossner P, Pastorkova A, Bavarova H, Frankova K, Anzion RB, Bos RP. 1994a. Urinary cyclophosphamide excretion and chromosomal aberrations in peripheral blood lymphocytes after occupational exposure to antineoplastic agents. Mutat Res. 309(2):193-199. http://dx.doi.org/10.1016/0027-5107(94)90092-2

Sessink PJ, Connor TH, Jorgenson JA, Tyler TG. 2011. Reduction in surface contamination with antineoplastic drugs in 22 hospital pharmacies in the US following implementation of a closedsystem drug transfer device. J Oncol Pharm Pract. 17(1):39-48.

http://dx.doi.org/10.1177/1078155210361431

Sessink PJ, Friemel NS, Anzion RB, Bos RP. 1994b. Biological and environmental monitoring of occupational exposure of pharmaceutical plant workers to methotrexate. Int Arch Occup Environ Health. 65(6):401-403. http://dx.doi.org/10.1007/BF00383251

Sessink PJ, Kroese ED, van Kranen HJ, Bos RP. 1995. Cancer risk assessment for health care workers occupationally exposed to cyclophosphamide. Int Arch Occup Environ Health. 67(5):317-323. http://dx.doi.org/10.1007/BF00385647

Sessink PJ, Leclercq GM, Wouters DM, Halbardier L, Hammad C, Kassoul N. 2015. Environmental contamination, product contamination and workers exposure using a robotic system for antineoplastic drug preparation. J Oncol Pharm Pract. 21(2):118-127. http://dx.doi.org/10.1177/1078155214522840

Sessink PJ, Trahan J, Coyne JW. 2013. Reduction in surface contamination with cyclophosphamide in 30 US hospital pharmacies following implementation of a closed-system drug transfer device. Hosp Pharm. 48(3):204-212. http://dx.doi.org/10.1310/hpj4803-204

Sessink PJ, Van de Kerkhof MC, Anzion RB, Noordhoek J, Bos RP. 1994c. Environmental contamination and assessment of exposure to antineoplastic agents by determination of cyclophosphamide in urine of exposed pharmacy technicians: Is skin absorption an important exposure route? Arch Environ Health. 49(3):165-169.

http://dx.doi.org/10.1080/00039896.1994.9940377

Sessink PJ, Verplanke AJ, Herber RF, Bos RP. 1997a. Occupational exposure to antineoplastic agents and parameters for renal dysfunction. Int Arch Occup Environ Health. 69(3):215-218. http://dx.doi.org/10.1007/s004200050139

Sessink PJ, Wittenhorst BC, Anzion RB, Bos RP. 1997b. Exposure of pharmacy technicians to antineoplastic agents: Reevaluation after additional protective measures. Arch Environ Health. 52(3):240-244. http://dx.doi.org/10.1080/00039899709602893

Sessink PJM, Joost HCdR, Pierik FH, Anzion RBM, Bos RP. 1993. Occupational exposure of animal caretakers to cyclophosphamide. J Occup Med. 35(1):47-52.

Shahrasbi AA, Afshar M, Shokraneh F, Monji F, Noroozi M, Ebrahimi-Khojin M, Madani SF, Ahadi-Barzoki M, Rajabi M. 2014. Risks to health professionals from hazardous drugs in Iran: A 
pilot study of understanding of healthcare team to occupational exposure to cytotoxics. EXCLI Journal. 13:491-501.

Shepard TH, Lemire RJ. 2004. Catalog of Teratogenic Agents. Baltimore, MD: The Johns Hopkins University Press.

Shortridge LA, Lemasters GK, Valanis B, Hertzberg V. 1995. Menstrual cycles in nurses handling antineoplastic drugs. Cancer Nurs. 18(6):439-444. http://dx.doi.org/10.1097/00002820$\underline{199512000-00003}$

Sibaud V, Lamant L, Maisongrosse V, Delord JP. 2013. [Adverse skin reactions induced by BRAF inhibitors: a systematic review]. Ann Dermatol Venereol. 140(8-9):510-520. http://dx.doi.org/10.1016/j.annder.2013.02.031

Siderov J, Kirsa S, McLauchlan R. 2009. Surface contamination of cytotoxic chemotherapy preparation areas in Australian Hospital Pharmacy Departments. J Pharm Pract Res. 39(2):117121. http://dx.doi.org/10.1002/j.2055-2335.2009.tb00434.x

Sill H, Olipitz W, Zebisch A, Schulz E, Wolfler A. 2011. Therapy-related myeloid neoplasms: pathobiology and clinical characteristics. Br J Pharmacol. 162(4):792-805.

http://dx.doi.org/10.1111/j.1476-5381.2010.01100.x

Skov T, Maarup B, Olsen J, Rorth M, Winthereik H, Lynge E. 1992. Leukaemia and reproductive outcome among nurses handling antineoplastic drugs. Brit J Ind Med. 49(12):855861.

Sorsa M, Pyy L, Salomaa S, Nylund L, Yager JW. 1988. Biological and environmental monitoring of occupational exposure to cyclophosphamide in industry and hospitals. Mutat Res. 204(3):465-479. http://dx.doi.org/10.1016/0165-1218(88)90042-0

Sottani C, Porro B, Comelli M, Imbriani M, Minoia C. 2010. An analysis to study trends in occupational exposure to antineoplastic drugs among health care workers. J Chromatogr B Analyt Technol Biomed Life Sci. 878(27):2593-2605.

http://dx.doi.org/10.1016/j.jchromb.2010.04.030

Sottani C, Porro B, Imbriani M, Minoia C. 2012. Occupational exposure to antineoplastic drugs in four Italian health care settings. Toxicol Lett. 213(1):107-115.

http://dx.doi.org/10.1016/j.toxlet.2011.03.028

Sottani C, Rinaldi P, Leoni E, Poggi G, Teragni C, Delmonte A, Minoia C. 2008. Simultaneous determination of cyclophosphamide, ifosfamide, doxorubicin, epirubicin and daunorubicin in human urine using high-performance liquid chromatography/electrospray ionization tandem mass spectrometry: Bioanalytical method validation. Rapid Commun Mass Spectrom.

22(17):2645-2659. http://dx.doi.org/10.1002/rcm.3657

Spatari G, Fenga C, Minciullo PL, Di Pasquale G, Cacciola A, Ventura-Spagnolo E, Gangemi S. 2005. Modification of interleukin-15 serum levels in workers exposed to chemotherapeutic agents. Mediators Inflamm. 2005(1):60-62. http://dx.doi.org/10.1155/MI.2005.60

Stiller A, Obe G, Boll I, Pribilla W. 1983. No elevation of the frequencies of chromosomal alterations as a consequence of handling cytostatic drugs. Analyses with peripheral blood and 
urine of hospital personnel. Mutat Res. 121(3-4):253-259. http://dx.doi.org/10.1016/0165$\underline{7992(83) 90211-7}$

Stücker I, Caillard JF, Collin R, Gout M, Poyen D, Hemon D. 1990. Risk of spontaneous abortion among nurses handling antineoplastic drugs. Scand J Work Environ Health. 16(2):102107. http://dx.doi.org/10.5271/sjweh.1811

Stücker I, Hirsch A, Doloy T, Bastie-Sigeac I, Hemon D. 1986. Urine mutagenicity, chromosomal abnormalities and sister chromatid exchanges in lymphocytes of nurses handling cytostatic drugs. Int Arch Occup Environ Health. 57(3):195-205.

http://dx.doi.org/10.1007/BF00405788

Stücker I, Mandereau L, Hemon D. 1993. Relationship between birthweight and occupational exposure to cytostatic drugs during or before pregnancy. Scand J Work Environ Health. 19(3):148-153. http://dx.doi.org/10.5271/sjweh.1488

Sugiura S, Asano M, Kinoshita K, Tanimura M, Nabeshima T. 2011a. Risks to health professionals from hazardous drugs in Japan: A pilot study of environmental and biological monitoring of occupational exposure to cyclophosphamide. J Oncol Pharm Pract. 17(1):14-19. https://doi.org/10.1177/1078155209358632

Sugiura S, Nakanishi H, Asano M, Hashida T, Tanimura M, Hama T, Nabeshima T. 2011b. Multicenter study for environmental and biological monitoring of occupational exposure to cyclophosphamide in Japan. J Oncol Pharm Pract. 17(1):20-28. http://dx.doi.org/10.1177/1078155210369851

Suspiro A, Prista J. 2011. Biomarkers of occupational exposure do anticancer agents: a minireview. Toxicol Lett. 207(1):42-52. http://dx.doi.org/10.1016/j.toxlet.2011.08.022

Tanimura M, Yamada K, Sugiura S, Mori K, Nagata H, Tadokoro K, Miyake T, Hamaguchi Y, Sessink P, Nabeshima T. 2009. An environmental and biological study of occupational exposure to cyclophosphamide in the pharmacy of a japanese community hospital designated for the treatment of cancer. J Health Sci. 55(5):750-756. http://dx.doi.org/10.1248/jhs.55.750

Taskinen H, Kyyronen P, Hemminki K, Hoikkala M, Lajunen K, Lindbohm ML. 1994. Laboratory work and pregnancy outcome. J Occup Environ Med. 36(3):311-319. http://dx.doi.org/10.1097/00043764-199403000-00008

Taskinen H, Lindbohm ML, Hemminki K. 1986. Spontaneous abortions among women working in the pharmaceutical-industry. Brit J Ind Med. 43(3):199-205.

Teschke K, Olshan AF, Daniels JL, De Roos AJ, Parks CG, Schulz M, Vaughan TL. 2002. Occupational exposure assessment in case-control studies: opportunities for improvement. Occup Environ Med. 59(9):575-593; discussion 594. http://dx.doi.org/10.1136/oem.59.9.575

Testa A, Giachelia M, Palma S, Appolloni M, Padua L, Tranfo G, Spagnoli M, Tirindelli D, Cozzi R. 2007. Occupational exposure to antineoplastic agents induces a high level of chromosome damage. Lack of an effect of GST polymorphisms. Toxicol Appl Pharmacol. 223(1):46-55. http://dx.doi.org/10.1016/j.taap.2007.05.006 
Thiringer G, Granung G, Holmen A, Hogstedt B, Jarvholm B, Jonsson D, Persson L, Wahlstrom J, Westin J. 1991. Comparison of methods for the biomonitoring of nurses handling antitumor drugs. Scand J Work Environ Health. 17(2):133-138. http://dx.doi.org/10.5271/sjweh.1724

Thulin H, Sundberg E, Hansson K, Cole J, Hartley-Asp B. 1995. Occupational exposure to nornitrogen mustard: Chemical and biological monitoring. Toxicol Ind Health. 11(1):89-97. http://dx.doi.org/10.1177/074823379501100108

Tigha Bouaziz N, Tourab D, Nezzal AM. 2016. Study of cardiovascular morbidity in nurses exposed to cytostatic drugs: Multivaried approach analysis. Ann Cardiol Angeiol. 65(3):179-184. http://dx.doi.org/10.1016/j.ancard.2016.04.014

Tompa A, Biro A, Jakab M. 2016. Genotoxic monitoring of nurses handling cytotoxic drugs. Asia Pac J Oncol Nurs. 3(4):365-369. http://dx.doi.org/10.4103/2347-5625.196484

Tompa A, Jakab M, Biro A, Magyar B, Fodor Z, Klupp T, Major J. 2006a. Chemical safety and health conditions among Hungarian hospital nurses. Ann N Y Acad Sci. 1076:635-648.

http://dx.doi.org/10.1196/annals.1371.054

Tompa A, Jakab M, Biro A, Major J. 2015. [Genetic and immune-toxicologic studies on abnormal thyroid functions in hospital employees exposed to cytostatic drugs]. Orv Hetil. 156(2):60-66. http://dx.doi.org/10.1556/oh.2015.30064

Tompa A, Magyar B, Toth F, Biro A, Fodor Z, Jakab M, Major J. 2006b. [The state of health of oncology nurses characterized by genetic and immunotoxicologic biomarkers]. Magy Onkol. 50(2):153-161. http://dx.doi.org/Huon.2006.50.2.0153

Touzin K, Bussieres JF, Langlois É, Lefebvre M. 2009. Evaluation of surface contamination in a hospital hematology-oncology pharmacy. J Onc Pharm Pract. 15(1):53-61. http://dx.doi.org/10.1177/1078155208096904

Turci R, Minoia C, Sottani C, Coghi R, Severi P, Castriotta C, Del Bianco M, Imbriani M. 2011. Occupational exposure to antineoplastic drugs in seven Italian hospitals: The effect of quality assurance and adherence to guidelines. J Oncol Pharm Pract. 17(4):320-332. http://dx.doi.org/10.1177/1078155210381931

Turci R, Sottani C, Ronchi A, Minoia C. 2002. Biological monitoring of hospital personnel occupationally exposed to antineoplastic agents. Toxicol Lett. 134(1-3):57-64. http://dx.doi.org/10.1016/S0378-4274(02)00163-7

Ündeğer U, Basaran N, Kars A, Guc D. 1999. Assessment of DNA damage in nurses handling antineoplastic drugs by the alkaline COMET assay. Mutat Res. 439(2):277-285. http://dx.doi.org/10.1016/S1383-5718(99)00002-9

Ursini CL, Cavallo D, Colombi A, Giglio M, Marinaccio A, Iavicoli S. 2006. Evaluation of early DNA damage in healthcare workers handling antineoplastic drugs. Int Arch Occup Environ Health. 80(2):134-140. http://dx.doi.org/10.1007/s00420-006-0111-X

Valanis B, Vollmer W, Labuhn K, Glass A. 1997. Occupational exposure to antineoplastic agents and self-reported infertility among nurses and pharmacists. J Occup Environ Med. 39(6):574-580. http://dx.doi.org/10.1097/00043764-199706000-00013 
Valanis B, Vollmer WM, Steele P. 1999. Occupational exposure to antineoplastic agents: Selfreported miscarriages and stillbirths among nurses and pharmacists. J Occup Environ Med. 41(8):632-638. http://dx.doi.org/10.1097/00043764-199908000-00004

Valanis BG, Hertzberg V, Shortridge L. 1987. Antineoplastic drugs. Handle with care. AAOHN J. 35(11):487-492.

Valanis BG, Vollmer WM, Labuhn KT, Glass AG. 1993a. Acute symptoms associated with antineoplastic drug handling among nurses. Cancer Nurs. 16(4):288-295.

http://dx.doi.org/10.1097/00002820-199308000-00005

Valanis BG, Vollmer WM, Labuhn KT, Glass AG. 1993b. Association of antineoplastic drug handling with acute adverse effects in pharmacy personnel. Am J Hosp Pharm. 50(3):455-462.

Valverde M, Rojas E. 2009. Environmental and occupational biomonitoring using the Comet assay. Mutat Res. 681(1):93-109. http://dx.doi.org/10.1016/j.mrrev.2008.11.001

Van Calsteren K, Heyns L, De Smet F, Van Eycken L, Gziri MM, Van Gemert W, Halaska M, Vergote I, Ottevanger N, F A. 2010. Cancer during pregnancy: an analysis of 215 patients emphasizing the obstetrical and the neonatal outcomes. J Clin Oncol. 28(4):683-689.

http://dx.doi.org/10.1200/JCO.2009.23.2801

Vandenbroucke J, Robays H. 2001. How to protect environment and employees against cytotoxic agents, the UZ Ghent experience. J Oncol Pharm Practice. 6:146-152.

http://dx.doi.org/10.1177/107815520100600403

Villa AF, El Balkhi S, Aboura R, Sageot H, Hasni-Pichard H, Pocard M, Elias D, Joly N, Payen D, Blot F et al. 2015. Evaluation of oxaliplatin exposure of healthcare workers during heated intraperitoneal perioperative chemotherapy (HIPEC). Ind Health. 53(1):28-37.

http://dx.doi.org/10.2486/indhealth.2014-0025

Villarini M, Dominici L, Fatigoni C, Muzi G, Monarca S, Moretti M. 2012. Biological effect monitoring in peripheral blood lymphocytes from subjects occupationally exposed to antineoplastic drugs: assessment of micronuclei frequency. J Occup Health. 54(6):405-415. http://dx.doi.org/10.1539/joh.12-0038-OA

Villarini M, Dominici L, Piccinini R, Fatigoni C, Ambrogi M, Curti G, Morucci P, Muzi G, Monarca S, Moretti M. 2011. Assessment of primary, oxidative and excision repaired DNA damage in hospital personnel handling antineoplastic drugs. Mutagenesis. 26(3):359-369. http://dx.doi.org/10.1093/mutage/geq102

Villarini M, Gianfredi V, Levorato S, Vannini S, Salvatori T, Moretti M. 2016. Occupational exposure to cytostatic/antineoplastic drugs and cytogenetic damage measured using the lymphocyte cytokinesis-block micronucleus assay: A systematic review of the literature and meta-analysis. Mutat Res. 770(Pt A):35-45. http://dx.doi.org/10.1016/j.mrrev.2016.05.001

Waksvik H, Klepp O, Brogger A. 1981. Chromosome analyses of nurses handling cytostatic agents. Cancer Treat Rep. 65(7-8):607-610. 
Walusiak J, Wagrowska-Koski E, Palczynski C. 2003. [Health effects of occupational exposure to cytostatics in medicinal personnel in the light of compulsory prophylactics: a cross-sectional study]. Med Pr. 54(3):229-236.

Wick C, Slawson MH, Jorgenson JA, Tyler LS. 2003. Using a closed-system protective device to reduce personnel exposure to antineoplastic agents. Am J Health Syst Pharm. 60(22):2314-2320.

Yager JW, Sorsa M, Selvin S. 1988. Micronuclei in cytokinesis-blocked lymphocytes as an index of occupational exposure to alkylating cytostatic drugs. IARC Sci Publ. (89):213-216.

Yang D, Xu S, Wang J. 2002a. [The study of DNA damage of peripheral lymphocytes in the nurses occupationally exposed to anticancer drugs]. Zhonghua Lao Dong Wei Sheng Zhi Ye Bing Za Zhi. 20(3):197-199.

Yang DP, Xu SJ, Wang JX. 2002b. Study on chromosome damage among nurses occupationally exposed to antineoplastic drugs in an oncology department. Biomed Environ Sci. 15(3):268-270.

Yin H, Xu XQ, Lin LY. 2010. [DNA damage of antineoplastic drugs exposure on nurses]. Zhonghua Lao Dong Wei Sheng Zhi Ye Bing Za Zhi. 28(3):218-219.

Yoshida J, Koda S, Nishida S, Nakano H, Tei G, Kumagai S. 2013. Association between occupational exposure and control measures for antineoplastic drugs in a pharmacy of a hospital. Ann Occup Hyg. 57(2):251-260. https://doi.org/10.1093/annhyg/mes061

Yoshida J, Kosaka H, Tomioka K, Kumagai S. 2006. Genotoxic risks to nurses from contamination of the work environment with antineoplastic drugs in Japan. J Occup Health. 48(6):517-522. http://dx.doi.org/10.1539/joh.48.517

Yoshida J, Tei G, Mochizuki C, Masu Y, Koda S, Kumagai S. 2009. Use of a closed system device to reduce occupational contamination and exposure to antineoplastic drugs in the hospital work environment. Ann Occup Hyg. 53(2):153-160. http://dx.doi.org/10.1093/annhyg/men081

Yuki M, Ishida T, Sekine S. 2015. Secondary exposure of family members to cyclophosphamide after chemotherapy of outpatients with cancer: A pilot study. Oncol Nurs Forum. 42(6):665-671. http://dx.doi.org/10.1188/15.ONF.42-06AP

Zack E. 2012. Chemotherapy and biotherapeutic agents for autoimmune diseases. Clin J Oncol Nurs. 16(4):E125-132. http://dx.doi.org/10.1188/12.CJON.E125-E132

Zare Sakhvidi MJ, Hajaghazadeh M, Mostaghaci M, Mehrparvar AH, Zare Sakhvidi F, Naghshineh E. 2016. Applicability of the comet assay in evaluation of DNA damage in healthcare providers' working with antineoplastic drugs: a systematic review and meta-analysis. Int J Occup Environ Health. 22(1):52-67. http://dx.doi.org/10.1080/10773525.2015.1123380

Zhang X, Zheng Q, Lv Y, An M, Zhang Y, Wei Y, Feng W. 2016. Evaluation of adverse health risks associated with antineoplastic drug exposure in nurses at two Chinese hospitals: The effects of implementing a pharmacy intravenous admixture service. Am J Ind Med. 59(4):264-273. http://dx.doi.org/10.1002/ajim.22553

Ziegler E, Mason HJ, Baxter PJ. 2002. Occupational exposure to cytotoxic drugs in two UK oncology wards. Occup Environ Med. 59(9):608-612. http://dx.doi.org/10.1136/oem.59.9.608 


\section{Appendix A. Literature Search Strategy}

The strategy for this search is broad for the consideration of adverse health outcomes, including biomarkers of effect, and comprehensive for occupational exposure to cancer chemotherapy agents as an exposure or treatment in order to ensure inclusion of relevant papers.

Table A-1. Literature Search Strategy

\begin{tabular}{|c|c|}
\hline Database & Search Terms \\
\hline Embase & $\begin{array}{l}\text { ('Antineoplastic agent'/exp OR 'antineoplastic agent') AND ('health care personnel'/exp OR 'health care } \\
\text { personnel') AND ('occupational exposure'/de OR 'occupational exposure' OR 'occupational hazard'/de OR } \\
\text { 'occupational hazard' OR 'occupational safety'/de OR 'occupational safety') AND ([embase]/lim OR } \\
\text { [embase classic]/lim) }\end{array}$ \\
\hline PubMed & 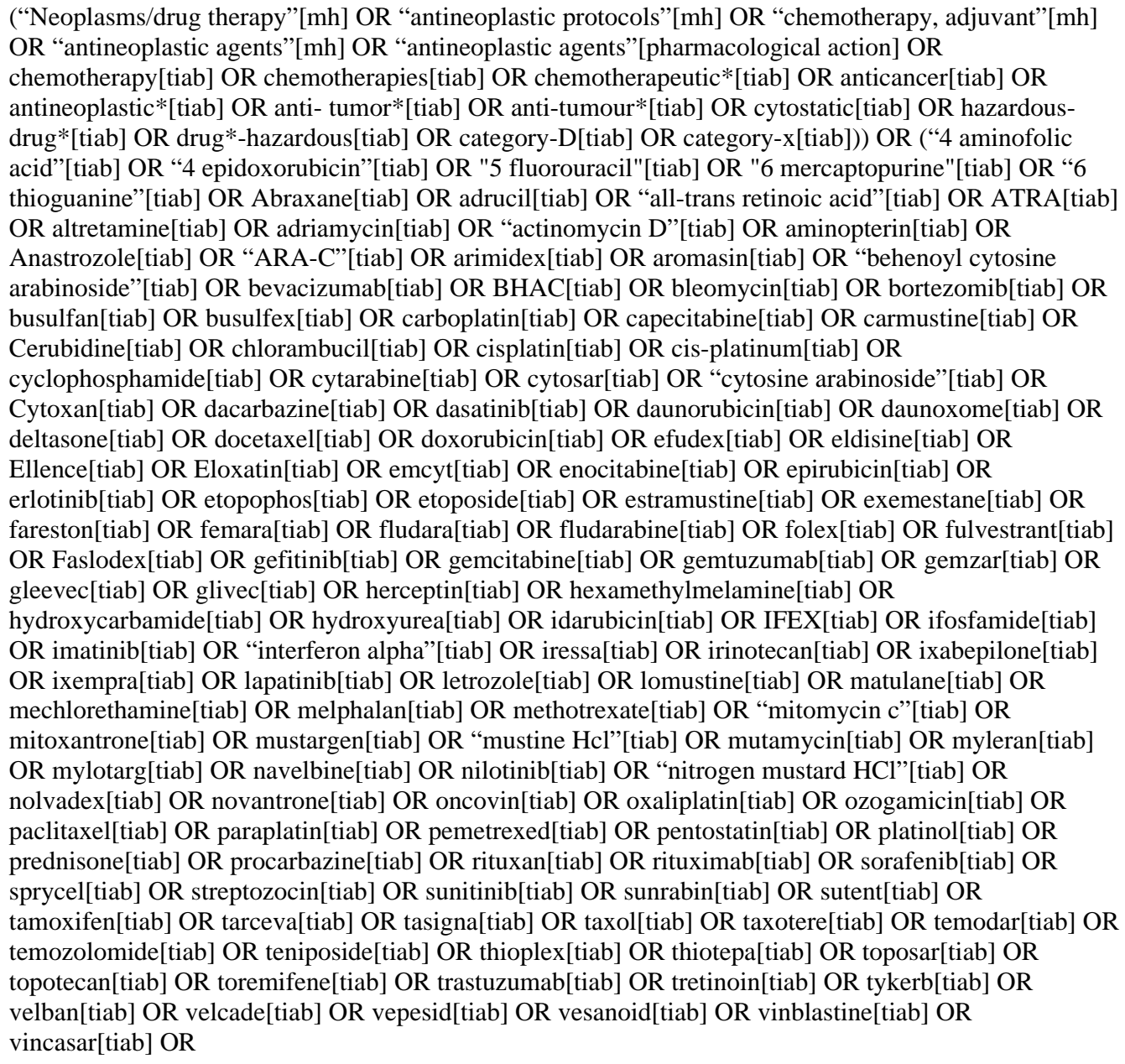 \\
\hline
\end{tabular}


vincrex[tiab] OR vincristine[tiab] OR vindesine[tiab] OR vinorelbine[tiab] OR VM26[tiab] OR VP16[tiab] OR Vumon[tiab] OR Xeloda[tiab] OR zanosar[tiab])

\section{AND}

(Environmental exposure[mh] OR Occupational exposure[mh] OR occupational diseases[mh] OR occupation*[tiab] OR workplace[tiab] OR work-related[tiab] OR exposure*[tiab] OR exposed[tiab] OR contaminat*[tiab] OR handl*[tiab])

\section{AND}

Health services[mh] OR health occupations[mh] OR health personnel[mh] OR pharmacist*[tiab] OR technician*[tiab] OR nurse*[tiab] OR nursing[tiab] OR physician*[tiab] OR clinician*[tiab] OR doctor*[tiab] OR veterinarian*[tiab])) OR ((hospital[tiab] OR clinic[tiab] OR medical[tiab] OR health[tiab] OR healthcare[tiab] OR health-care[tiab] OR pharmacy[tiab] OR pharmaceutical*[tiab] OR cancer[tiab] OR oncolog*[tiab] OR veterinary[tiab]) AND (staff[tiab] OR personnel[tiab] OR worker*[tiab] OR employee*[tiab] OR technician*[tiab] OR aide*[tiab] OR assistant*[tiab] OR professional*[tiab] OR setting*[tiab]

\section{AND}

(Neoplasms[mh] OR neoplas*[tiab] OR cancer[tiab] OR cancerous[tiab] OR carcinogenic*[tiab] OR tumor[tiab] OR tumors[tiab] OR tumorigen*[tiab] OR tumour*[tiab] OR leukemia*[tiab] OR lymphoma*[tiab] OR sarcoma*[tiab] OR carcinoma*[tiab] OR adenoma*[tiab] OR melanoma*[tiab] OR DNA damage[mh] OR dna-damage[tiab] OR genetic-damage[tiab] OR chromosom*[tiab] OR chromatid[tiab] OR mutagenicity tests[mh] OR mutagen*[tiab] OR genotox*[tiab] OR micronucle*[tiab]) OR (Reproduction[mh] OR reproduction[tiab] OR reproductive[tiab] OR sexual[tiab] OR intercourse[tiab] OR coitus[tiab] OR ejaculat*[tiab] OR orgasm*[tiab] OR fertiliz*[tiab] OR conception[tiab] OR gametogenesis[tiab] OR oogenesis[tiab] OR spermatogenesis[tiab] OR inseminat*[tiab] OR luteinization[tiab] OR ovulat*[tiab] OR anovulat*[tiab] OR superovulat*[tiab] OR erection*[tiab] OR Reproductive physiological phenomena[mh] OR andropause[tiab] OR menopause[tiab] OR estrous[tiab] OR fertility[tiab] OR fecund*[tiab] OR time-to-pregnancy[tiab] OR menstrua*[tiab] OR pubert*[tiab] OR adrenarche[tiab] OR menarche[tiab] OR Genital diseases, female[mh] OR endometriosis[tiab] OR infertil*[tiab] OR pelvic-inflamm*[tiab] OR genital diseases, male[mh] OR epididymitis[tiab] OR hypospadia*[tiab] OR priapism[tiab] OR prostatitis[tiab] OR cryptorchidism[tiab] OR varicocele[tiab] OR erectile-dysfunction[tiab] OR Pregnancy[mh] OR Pregnant[tiab] OR pregnancy[tiab] OR pregnancies[tiab] OR obstetric*[tiab] OR maternal*[tiab] OR mother*[tiab] OR embryonic and fetal development[mh] OR embryonic-development[tiab] OR fetaldevelopment[tiab] OR organogenesis[tiab] OR body size[mh] OR birth-weight*[tiab] OR growthretardation[tiab] OR small-for-gestation*[tiab] OR embryo[tiab] OR embryos[tiab] OR embryonic[tiab] OR fetus[tiab] OR foetus[tiab] OR fetal[tiab] OR foetal[tiab] OR gestation*[tiab] OR peripartum[tiab] OR postpartum[tiab] OR placent*[tiab] OR prenatal[tiab] OR perinat*[tiab] OR neonat*[tiab] OR postnat*[tiab] OR labor-onset[tiab] OR Parturition*[tiab] OR birth[tiab] OR births[tiab] OR childbirth*[tiab] OR gravidity[tiab] OR parity[tiab] OR Pregnancy complications[mh] OR pregnancycomplication*[tiab] OR complicated-pregnan*[tiab] OR labor-complication*[tiab] OR complicatedlabor[tiab] OR gestational-diabetes[tiab] OR (pregnancy[tiab] AND hypertension[tiab]) OR maternaldeath[tiab] OR morning-sickness[tiab] OR breech[tiab] OR ectopic[tiab] OR Pregnancy outcome[mh] OR pregnancy-outcome*[tiab] OR live-birth*[tiab] OR full-term[tiab] OR term-birth*[tiab] OR newborn*[tiab] OR baby[tiab] OR babies[tiab] OR infant*[tiab] OR premature[tiab] OR preterm[tiab] OR pre-term[tiab] OR stillbirth*[tiab] OR still-birth*[tiab] OR stillborn*[tiab] OR still-born*[tiab] OR spontaneous-abortion*[tiab] OR miscarriage*[tiab] OR infant mortality[mh] OR infant-mortality[tiab] OR embryo*-loss[tiab] OR congenital, hereditary, and neonatal diseases and abnormalities[mh] OR congenital[tiab] OR abnormal*[tiab] OR malform*[tiab] OR retard*[tiab] OR prenatal exposure delayed effects[mh] OR teratogen*[tiab] OR embryotoxic*[tiab]) 


\section{Database}

\section{Search Terms}

Scopus

TOXLINE xeloda OR zanosar)

AND AND OR assistant* OR professional* OR setting*))
TITLE-ABS-KEY(chemotherapy* OR anticancer OR antineoplastic* OR anti-tumor* OR anti-tumour* OR cytostatic OR hazardous-drug* OR drug*-hazardous OR category-d OR category-x OR "4 aminofolic acid" OR "4 epidoxorubicin" OR "5 fluorouracil" OR "6 mercaptopurine" OR "6 thioguanine" OR abraxane OR adrucil OR "all-trans retinoic acid" OR atra OR altretamine OR adriamycin OR "actinomycin D" OR aminopterin OR anastrozole OR "ARA-C" OR arimidex OR aromasin OR "behenoyl cytosine arabinoside" OR bevacizumab OR bhac OR bleomycin OR bortezomib OR busulfan OR busulfex OR carboplatin OR capecitabine OR carmustine OR cerubidine OR chlorambucil OR cisplatin OR cis-platinum OR cyclophosphamide OR cytarabine OR cytosar OR "cytosine arabinoside" OR cytoxan OR dacarbazine OR dasatinib OR daunorubicin OR daunoxome OR deltasone OR docetaxel OR doxorubicin OR efudex OR eldisine OR ellence OR eloxatin OR emcyt OR enocitabine OR epirubicin OR erlotinib OR etopophos OR etoposide OR estramustine OR exemestane OR fareston OR femara OR fludara OR fludarabine OR folex OR fulvestrant OR faslodex OR gefitinib OR gemcitabine OR gemtuzumab OR gemzar OR gleevec OR glivec OR herceptin OR hexamethylmelamine OR hydroxycarbamide OR hydroxyurea OR idarubicin OR ifex OR ifosfamide OR imatinib OR "interferon alpha" OR iressa OR irinotecan OR ixabepilone OR ixempra OR lapatinib OR letrozole OR lomustine OR matulane OR mechlorethamine OR melphalan OR methotrexate OR "mitomycin c" OR mitoxantrone OR mustargen OR "mustine Hcl" OR mutamycin OR myleran OR mylotarg OR navelbine OR nilotinib OR "nitrogen mustard HCl" OR nolvadex OR novantrone OR oncovin OR oxaliplatin OR ozogamicin OR paclitaxel OR paraplatin OR pemetrexed OR pentostatin OR platinol OR prednisone OR procarbazine OR rituxan OR rituximab OR sorafenib OR sprycel OR streptozocin OR sunitinib OR sunrabin OR sutent OR tamoxifen OR tarceva OR tasigna OR taxol OR taxotere OR temodar OR temozolomide OR teniposide OR thioplex OR thiotepa OR toposar OR topotecan OR toremifene OR trastuzumab OR tretinoin OR tykerb OR velban OR velcade OR vepesid OR vesanoid OR vinblastine OR vincasar OR vincrex OR vincristine OR vindesine OR vinorelbine OR vm26 OR vp16 OR vumon OR

TITLE-ABS-KEY(occupation* OR workplace OR work-related OR contaminat* OR handl*)

TITLE-ABS-KEY((pharmacist* OR technician* OR nurse* OR nursing OR physician* OR clinician* OR doctor* OR veterinarian* OR hospital OR clinic OR medical OR health OR healthcare OR healthcare OR pharmacy OR pharmaceutical* OR cancer OR oncolog* OR veterinary) AND (staff OR personnel OR worker* OR employee* OR technician* OR aide*

(chemotherapy OR chemotherapies OR chemotherapeutic OR anticancer OR "anti cancer" OR antineoplastic* OR "anti tumor" OR "anti tumorigenic" OR "anti tumour" OR “anti tumourigenic” OR cytostatic)

\section{AND}

(occupation* OR workplace OR work related OR contaminat* OR handl*)

\section{AND}

(pharmacist OR pharmacists OR technician OR nurse OR nursing OR physician OR clinician OR doctor OR veterinarian OR hospital OR clinic OR medical OR health OR healthcare OR health care OR pharmacy OR pharmaceutical OR cancer OR oncolog* OR veterinary) AND (staff OR personnel OR worker OR employee OR technician OR aide OR assistant OR professional OR setting) 
Database

Web of Science

\section{Search Terms}

Topic $=$ (Chemotherap* OR anticancer OR antineoplastic* OR anti- tumor* OR anti-tumour* OR cytostatic OR hazardous-drug* OR drug*-hazardous OR category-D OR category-x OR "4 aminofolic acid” OR “4 epidoxorubicin” OR "5 fluorouracil" OR "6 mercaptopurine" OR "6 thioguanine” OR Abraxane OR adrucil OR "all-trans retinoic acid” OR ATRA OR altretamine OR adriamycin OR “actinomycin D” OR aminopterin OR Anastrozole OR “ARA-C” OR arimidex OR aromasin OR "behenoyl cytosine arabinoside” OR bevacizumab OR BHAC OR bleomycin OR bortezomib OR busulfan OR busulfex OR carboplatin OR capecitabine OR carmustine OR Cerubidine OR chlorambucil OR cisplatin OR cis-platinum OR cyclophosphamide OR cytarabine OR cytosar OR “cytosine arabinoside” OR Cytoxan OR dacarbazine OR dasatinib OR daunorubicin OR daunoxome OR deltasone OR docetaxel OR doxorubicin OR efudex OR eldisine OR Ellence OR Eloxatin OR emcyt OR enocitabine OR epirubicin OR erlotinib OR etopophos OR etoposide OR estramustine OR exemestane OR fareston OR femara OR fludara OR fludarabine OR folex OR fulvestrant OR Faslodex OR gefitinib OR gemcitabine OR gemtuzumab OR gemzar OR gleevec OR glivec OR herceptin OR hexamethylmelamine OR hydroxycarbamide OR hydroxyurea OR idarubicin OR IFEX OR ifosfamide OR imatinib OR “interferon alpha” OR iressa OR irinotecan OR ixabepilone OR ixempra OR lapatinib OR letrozole OR lomustine OR matulane OR mechlorethamine OR melphalan OR methotrexate OR "mitomycin c" OR mitoxantrone OR mustargen OR “mustine Hcl” OR mutamycin OR myleran OR mylotarg OR navelbine OR nilotinib OR "nitrogen mustard HCl” OR nolvadex OR novantrone OR oncovin OR oxaliplatin OR ozogamicin OR paclitaxel OR paraplatin OR pemetrexed OR pentostatin OR platinol OR prednisone OR procarbazine OR rituxan OR rituximab OR sorafenib OR sprycel OR streptozocin OR sunitinib OR sunrabin OR sutent OR tamoxifen OR tarceva OR tasigna OR taxol OR taxotere OR temodar OR temozolomide OR teniposide OR thioplex OR thiotepa OR toposar OR topotecan OR toremifene OR trastuzumab OR tretinoin OR tykerb OR velban OR velcade OR vepesid OR vesanoid OR vinblastine OR vincasar OR vincrex OR vincristine OR vindesine OR vinorelbine OR VM26 OR VP16 OR Vumon OR Xeloda OR zanosar)

AND

Topic $=(($ pharmacist* OR technician* OR nurse* OR nursing OR physician* OR clinician* OR doctor* OR veterinarian* OR hospital OR clinic OR medical OR health OR healthcare OR health-care OR pharmacy OR pharmaceutical* OR cancer OR oncolog* OR veterinary) AND (staff OR personnel OR worker* OR employee* OR technician* OR aide* OR assistant* OR professional* OR setting*)) AND

Topic $=($ occupation* OR workplace OR work-related OR contaminat* OR handl*) AND

Topic $=$ (neoplas* OR cancer OR cancerous OR carcinogenic* OR tumor OR tumors OR tumorigen* OR tumour* OR leukemia* OR lymphoma* OR sarcoma* OR carcinoma* OR adenoma* OR melanoma* OR dna-damage OR genetic-damage OR chromosom* OR chromatid OR mutagen* OR genotox* OR micronucle*) OR Topic $=($ reproduction OR reproductive OR sexual OR intercourse OR coitus OR ejaculat* OR orgasm* OR fertiliz* OR conception OR gametogenesis OR oogenesis OR spermatogenesis OR inseminat* OR luteinization OR ovulat* OR anovulat* OR superovulat* OR erection* OR andropause OR menopause OR estrous OR fertility OR fecund* OR time-to-pregnancy OR menstrua* OR pubert* OR adrenarche OR menarche OR endometriosis OR infertil* OR pelvic-inflamm* OR epididymitis OR hypospadia* OR priapism OR prostatitis OR cryptorchidism OR varicocele OR erectiledysfunction OR pregnant OR pregnancy OR pregnancies OR obstetric* OR maternal* OR mother* OR embryonic-development OR fetal-development OR organogenesis OR “body size” OR birth-weight* OR growth-retardation OR small-for-gestation* OR embryo OR embryos OR embryonic OR fetus OR foetus OR fetal OR foetal OR gestation* OR peripartum OR postpartum OR placent* OR prenatal OR perinat* OR neonat* OR postnat* OR labor-onset OR Parturition* OR birth OR births OR childbirth* OR gravidity OR parity OR pregnancy-complication* OR complicated-pregnan* OR labor-complication* OR complicated-labor OR gestational-diabetes OR (pregnancy AND hypertension) OR maternal-death OR morning-sickness OR breech OR ectopic OR pregnancy-outcome* OR live-birth* OR full-term OR termbirth* OR newborn* OR baby OR babies OR infant* OR premature OR preterm OR pre-term OR stillbirth* OR still-birth* OR stillborn* OR still-born* OR spontaneous-abortion* OR miscarriage* OR infant-mortality OR embryo*-loss OR congenital OR abnormal* OR malform* OR retard* OR teratogen* OR embryotoxic*) 


\section{Appendix B. List of Included Studies Reporting Health Outcomes}

Anwar WA, Salama SI, el Serafy MM, Hemida SA, Hafez AS. 1994. Chromosomal aberrations and micronucleus frequency in nurses occupationally exposed to cytotoxic drugs. Mutagenesis. 9(4):315-317. http://dx.doi.org/10.1093/mutage/9.4.315

Biró A, Fodor Z, Major J, Tompa A. 2011. Immunotoxicity monitoring of hospital staff occupationally exposed to cytostatic drugs. Pathol Oncol Res. 17(2):301-308.

http://dx.doi.org/10.1007/s12253-010-9317-z

Bolognesi C, Nucci MC, Colacci AM, Grilli S, Ippoliti F, Mucci N, Romano Spica V, Barbieri A, Canitano N, Chiozzotto D et al. 2005. [Biomonitoring of nurses occupationally exposed to antineoplastic drugs: The IMEPA Project]. Epidemiol Prev. 29(5-6 Suppl):91-95.

Boughattas AB, Bouraoui S, Debbabi F, El Ghazel H, Saad A, Mrizak N. 2010. [Genotoxic risk assessment of nurses handling antineoplastic drugs]. Ann Biol Clin. 68(5):545-553.

http://dx.doi.org/10.1684/abc.2010.0464

Bouraoui S, Brahem A, Tabka F, Mrizek N, Saad A, Elghezal H. 2011. Assessment of chromosomal aberrations, micronuclei and proliferation rate index in peripheral lymphocytes from Tunisian nurses handling cytotoxic drugs. Environ Toxicol Pharmacol. 31(1):250-257. http://dx.doi.org/10.1016/j.etap.2010.11.004

Bouyer J, Saurel-Cubizolles MJ, Grenier C, Aussel L, Job-Spira N. 1998. Ectopic pregnancy and occupational exposure of hospital personnel. Scand J Work Environ Health. 24(2):98-103. http://dx.doi.org/10.5271/sjweh.285

Buono-Michel M, Orsière T, Sari-Minodier I, Botta A. 2000. Cytogenetic biomonitoring of nurses handling cytostatics. Arch Mal Prof. 61(3):148-155.

Burgaz S, Karahalil B, Bayrak P, Taskin L, Yavuzaslan F, Bokesoy I, Anzion RB, Bos RP, Platin N. 1999. Urinary cyclophosphamide excretion and micronuclei frequencies in peripheral lymphocytes and in exfoliated buccal epithelial cells of nurses handling antineoplastics. Mutat Res. 439(1):97-104. http://dx.doi.org/10.1016/S1383-5718(98)00180-6

Burgaz S, Karahalil B, Canhi Z, Terzioglu F, Ancel G, Anzion RB, Bos RP, Huttner E. 2002. Assessment of genotoxic damage in nurses occupationally exposed to antineoplastics by the analysis of chromosomal aberrations. Hum Exp Toxicol. 21(3):129-135.

http://dx.doi.org/10.1191/0960327102ht230oa

Buschini A, Villarini M, Feretti D, Mussi F, Dominici L, Zerbini I, Moretti M, Ceretti E, Bonfiglioli R, Carrieri M et al. 2013. Multicentre study for the evaluation of mutagenic/carcinogenic risk in nurses exposed to antineoplastic drugs: Assessment of DNA damage. Occup Environ Med. 70(11):789-794. http://dx.doi.org/10.1136/oemed-2013-101475

Caciari T, Casale T, Tomei F, Samperi I, Tomei G, Capozzella A, Ripamonti K, Scala B, Andreozzi G, Nardone $\mathrm{N}$ et al. 2012. Exposure to antineoplastic drugs in health care and blood chemistry parameters. Clin Ter. 163(6):e387-392. 
Cavallo D, Ursini CL, Perniconi B, Francesco AD, Giglio M, Rubino FM, Marinaccio A, Iavicoli S. 2005. Evaluation of genotoxic effects induced by exposure to antineoplastic drugs in lymphocytes and exfoliated buccal cells of oncology nurses and pharmacy employees. Mutat Res. 587(1-2):45-51. http://dx.doi.org/10.1016/j.mrgentox.2005.07.008

Connor TH, DeBord DG, Pretty JR, Oliver MS, Roth TS, Lees PS, Krieg EF, Jr., Rogers B, Escalante CP, Toennis CA et al. 2010. Evaluation of antineoplastic drug exposure of health care workers at three university-based US cancer centers. J Occup Environ Med. 52(10):1019-1027. http://dx.doi.org/10.1097/JOM.0b013e3181f72b63

Cooke J, Williams J, Morgan RJ, Cooke P, Calvert RT. 1991. Use of cytogenetic methods to determine mutagenic changes in the blood of pharmacy personnel and nurses who handle cytotoxic agents. Am J Hosp Pharm. 48(6):1199-1205.

Cornetta T, Pádua L, Testa A, Ievoli E, Festa F, Tranfo G, Baccelliere L, Cozzi R. 2008. Molecular biomonitoring of a population of nurses handling antineoplastic drugs. Mutat Res. 638(1-2):75-82. http://dx.doi.org/10.1016/j.mrfmmm.2007.08.017

Deng HP, Lou JL, Zhang MB, Wu W, Jin LF, Chen SJ, Zheng W, Wang BH, He JL. 2006. Detecting the cytogenetic effects in workers occupationally exposed to vincristine with four genetic tests. Mutat Res. 599(1-2):152-159. http://dx.doi.org/10.1016/j.mrfmmm.2006.02.003

Deng HP, Zhang MB, He JL, Wu W, Jin LF, Zheng W, Lou JL, Wang BH. 2005. Investigating genetic damage in workers occupationally exposed to methotrexate using three genetic endpoints. Mutagenesis. 20(5):351-357. http://dx.doi.org/10.1093/mutage/gei048

El-Ebiary AA, Abuelfadl AA, Sarhan NI. 2013. Evaluation of genotoxicity induced by exposure to antineoplastic drugs in lymphocytes of oncology nurses and pharmacists. J Appl Toxicol. 33(3):196-201. http://dx.doi.org/10.1002/jat.1735

Elshamy K, El-Hadidi M, El-Roby M, Fouda M. 2010. Health hazards among oncology nurses exposed to chemotherapy drugs. Afr J Haematol Oncol. 1(3):70-78.

Ensslin AS, Huber R, Pethran A, Rommelt H, Schierl R, Kulka U, Fruhmann G. 1997. Biological monitoring of hospital pharmacy personnel occupationally exposed to cytostatic drugs: Urinary excretion and cytogenetics studies. Int Arch Occup Environ Health. 70(3):205208. http://dx.doi.org/10.1007/s004200050208

Fransman W, Roeleveld N, Peelen S, de Kort W, Kromhout H, Heederik D. 2007. Nurses with dermal exposure to antineoplastic drugs: Reproductive outcomes. Epidemiology. 18(1):112-119. http://dx.doi.org/10.1097/01.ede.0000246827.44093.c1

Garaj-Vrhovac V, Kopjar N. 1998. Micronuclei in cytokinesis-blocked lymphocytes as an index of occupational exposure to antineoplastic drugs. Radiol Oncol. 32(4):385-392.

Grummt T, Grummt HJ, Schott G. 1993. Chromosomal aberrations in peripheral lymphocytes of nurses and physicians handling antineoplastic drugs. Mutat Res. 302(1):19-24.

http://dx.doi.org/10.1016/0165-7992(93)90085-A 
Gunnarsdóttir HK, Aspelund T, Karlsson T, Rafnsson VV. 1997. Occupational risk factors for breast cancer among nurses. Int J Occup Environ Health. 3(4):254-258. http://dx.doi.org/10.1179/oeh.1997.3.4.254

Harris PE, Connor TH, Stevens KR, Theiss JC. 1992. Cytogenetic assessment of occupational exposure of nurses to antineoplastic agents. J Occup Med Toxicol. 1(3):243-254.

Hemminki K, Kyyronen P, Lindbohm ML. 1985. Spontaneous abortions and malformations in the offspring of nurses exposed to anaesthetic gases, cytostatic drugs, and other potential hazards in hospitals, based on registered information of outcome. J Epidemiol Commun H. 39(2):141147. http://dx.doi.org/10.1136/jech.39.2.141

Hessel H, Radon K, Pethran A, Maisch B, Grobmair S, Sautter I, Fruhmann G. 2001. The genotoxic risk of hospital, pharmacy and medical personnel occupationally exposed to cytostatic drugs--evaluation by the micronucleus assay. Mutat Res. 497(1-2):101-109.

http://dx.doi.org/10.1016/S1383-5718(01)00236-4

Hola N, Sram RJ, Roznickova I. 1988. Evaluation of genotoxic risk in occupational exposure to cytostatics. Prac Lek. 40(4):154-157.

Izdes S, Sardas S, Kadioglu E, Kaymak C, Ozcagli E. 2009. Assessment of genotoxic damage in nurses occupationally exposed to anaesthetic gases or antineoplastic drugs by the comet assay. J Occup Health. 51(3):283-286. http://dx.doi.org/10.1080/152873901300018011

Jakab MG, Major J, Tompa A. 2001. Follow-up genotoxicological monitoring of nurses handling antineoplastic drugs. J Toxicol Environ Health A. 62(5):307-318.

http://dx.doi.org/10.1080/152873901300018011

Jeebhay M, Mbuli S, Uebel R. 1993. Assessment of exposure to chloramphenicol and azathioprine among workers in a South African pharmaceutical plant. Int Arch Occup Environ Health. 65(S1):S119-122. http://dx.doi.org/10.1007/BF00381321

Jochimsen PR, Corder MP, Lachenbruch PA, Spaight ME. 1988. Preparation and administration of chemotherapy. Haematological consequences for hospital-based nurses. Med Toxicol Adverse Drug Exp. 3(1):59-63. http://dx.doi.org/10.1007/BF03259931

Kašuba V, Rozgaj R, Garaj-Vrhovac V. 1999. Analysis of sister chromatid exchange and micronuclei in peripheral blood lymphocytes of nurses handling cytostatic drugs. J Appl Toxicol. 19(6):401-404. http://dx.doi.org/10.1002/(SICI)1099-1263(199911/12)19:6<401::AIDJAT592>3.0.CO;2-H

Kevekordes S, Gebel TW, Hellwig M, Dames W, Dunkelberg H. 1998. Human effect monitoring in cases of occupational exposure to antineoplastic drugs: A method comparison. Occup Environ Med. 55(3):145-149. http://dx.doi.org/10.1136/oem.55.3.145

Kopjar N, Garaj-Vrhovac V. 2001. Application of the alkaline comet assay in human biomonitoring for genotoxicity: A study on Croatian medical personnel handling antineoplastic drugs. Mutagenesis. 16(1):71-78. http://dx.doi.org/10.1093/mutage/16.1.71

Kopjar N, Garaj-Vrhovac V, Kasuba V, Rozgaj R, Ramic S, Pavlica V, Zeljezic D. 2009. Assessment of genotoxic risks in Croatian health care workers occupationally exposed to 
cytotoxic drugs: A multi-biomarker approach. Int J Hyg Environ Health. 212(4):414-431. http://dx.doi.org/10.1016/j.ijheh.2008.10.001

Krepinsky A, Bryant DW, Davison L, Young B, Heddle J, McCalla DR, Douglas G, Michalko K. 1990. Comparison of three assays for genetic effects of antineoplastic drugs on cancer patients and their nurses. Environ Mol Mutagen. 15(2):83-92.

http://dx.doi.org/10.1002/em.2850150205

Krstev S, Perunicic B, Vidakovic A. 2003. Work practice and some adverse health effects in nurses handling antineoplastic drugs. Med Lav. 94(5):432-439.

Ladeira C, Viegas S, Pádua M, Gomes M, Carolino E, Gomes MC, Brito M. 2014. Assessment of genotoxic effects in nurses handling cytostatic drugs. J Toxicol Environ Health A. 77(1416):879-887. http://dx.doi.org/10.1080/15287394.2014.910158

Laffon B, Teixeira JP, Silva S, Loureiro J, Torres J, Pasaro E, Mendez J, Mayan O. 2005. Genotoxic effects in a population of nurses handling antineoplastic drugs, and relationship with genetic polymorphisms in DNA repair enzymes. Am J Ind Med. 48(2):128-136.

http://dx.doi.org/10.1080/15287394.2014.910158

Lawson CC, Rocheleau CM, Whelan EA, Lividoti Hibert EN, Grajewski B, Spiegelman D, Rich-Edwards JW. 2012. Occupational exposures among nurses and risk of spontaneous abortion. Am J Obstet Gynecol. 206(4):327.e321-327.e328.

http://dx.doi.org/10.1016/j.ajog.2011.12.030

Lawson CC, Whelan EA, Hibert EN, Grajewski B, Spiegelman D, Rich-Edwards JW. 2009. Occupational factors and risk of preterm birth in nurses. Am J Obstet Gynecol. 200(1):51 e5158. http://dx.doi.org/10.1016/j.ajog.2008.08.006

Li ML, Zhang YY, Wang QX, Pei Y, Xiao CS. 2005. [Effects of antineoplastics drug on Tlymphocytes subsets in nurses of occupational exposure to antineoplastic drugs]. Zhonghua Lao Dong Wei Sheng Zhi Ye Bing Za Zhi. 23(6):450-451.

Lorente C, Cordier S, Bergeret A, De Walle HE, Goujard J, Ayme S, Knill-Jones R, Calzolari E, Bianchi F. 2000. Maternal occupational risk factors for oral clefts. Occupational Exposure and Congenital Malformation Working Group. Scand J Work Environ Health. 26(2):137-145. http://dx.doi.org/10.5271/sjweh.523

Machado-Santelli GM, Cerqueira EM, Oliveira CT, Pereira CA. 1994. Biomonitoring of nurses handling antineoplastic drugs. Mutat Res. 322(3):203-208. http://dx.doi.org/10.1016/0165$\underline{1218(94) 90007-8}$

Mader RM, Kokalj A, Kratochvil E, Pilger A, Rudiger HW. 2009. Longitudinal biomonitoring of nurses handling antineoplastic drugs. J Clin Nurs. 18(2):263-269.

http://dx.doi.org/10.1111/j.1365-2702.2007.02189.x

Mahmoodi M, Soleyman-Jahi S, Zendehdel K, Mozdarani H, Azimi C, Farzanfar F, Safari Z, Mohagheghi MA, Khaleghian M, Divsalar K et al. 2017. Chromosomal aberrations, sister chromatid exchanges, and micronuclei in lymphocytes of oncology department personnel 
handling anti-neoplastic drugs. Drug Chem Toxicol. 40(2):235-240.

http://dx.doi.org/10.1080/01480545.2016.1209678

Maluf SW, Erdtmann B. 2000. Follow-up study of the genetic damage in lymphocytes of pharmacists and nurses handling antineoplastic drugs evaluated by cytokinesis-block micronuclei analysis and single cell gel electrophoresis assay. Mutat Res. 471(1-2):21-27.

http://dx.doi.org/10.1016/S1383-5718(00)00107-8

McAbee RR, Gallucci BJ, Checkoway H. 1993. Adverse reproductive outcomes and occupational exposures among nurses: An investigation of multiple hazardous exposures. AAOHN J. 41(3):110-119.

McDiarmid MA, Oliver MS, Roth TS, Rogers B, Escalante C. 2010. Chromosome 5 and 7 abnormalities in oncology personnel handling anticancer drugs. J Occup Environ Med. 52(10):1028-1034. http://dx.doi.org/10.1097/JOM.0b013e3181f73ae6

McDiarmid MA, Rogers B, Oliver MS. 2014. Chromosomal effects of non-alkylating drug exposure in oncology personnel. Environ Mol Mutagen. 55(4):369-374.

http://dx.doi.org/10.1002/em.21852

McDonald AD, McDonald JC, Armstrong B, Cherry NM, Cote R, Lavoie J, Nolin AD, Robert D. 1988. Congenital defects and work in pregnancy. Brit J Ind Med. 45(9):581-588.

McDonald AD, McDonald JC, Armstrong B, Cherry NM, Cote R, Lavoie J, Nolin AD, Robert D. 1988. Fetal death and work in pregnancy. Brit J Ind Med. 45(3):148-157.

Medková J. 1990. Cytogenetic analysis of peripheral lymphocytes in occupationally exposed health personnel. Acta Univ Palacki Olomuc Fac Med. 126:93-106.

Medková J. 1991. An analysis of the health condition of personnel exposed to cytostatics at an oncology unit. Acta Univ Palacki Olomuc Fac Med. 130:311-322.

Milković-Kraus S, Horvat D. 1991. Chromosomal abnormalities among nurses occupationally exposed to antineoplastic drugs. Am J Ind Med. 19(6):771-774.

http://dx.doi.org/10.1002/ajim.4700190609

Moretti M, Grollino MG, Pavanello S, Bonfiglioli R, Villarini M, Appolloni M, Carrieri M, Sabatini L, Dominici L, Stronati L et al. 2015. Micronuclei and chromosome aberrations in subjects occupationally exposed to antineoplastic drugs: A multicentric approach. Int Arch Occup Environ Health. 88(6):683-695. http://dx.doi.org/10.1007/s00420-014-0993-y

Moretti M, Villarini M, Dominici L, Fatigoni C, Dell'omo M, Elisei E, Muzi G, Monarca S. 2013. [Evaluation of genotoxic effects in subjects occupationally exposed to antineoplastic drugs]. Ig Sanita Pubbl. 69(1):55-77.

Mrđanović J, Jungic S, Solajic S, Bogdanovic V, Jurisic V. 2012. Effects of orally administered antioxidants on micronuclei and sister chromatid exchange frequency in workers professionally exposed to antineoplastic agents. Food Chem Toxicol. 50(8):2937-2944.

http://dx.doi.org/10.1016/j.fct.2012.04.027 
Mušák L, Smerhovsky Z, Halasova E, Osina O, Letkova L, Vodickova L, Polakova V, Buchancova J, Hemminki K, Vodicka P. 2013. Chromosomal damage among medical staff occupationally exposed to volatile anesthetics, antineoplastic drugs, and formaldehyde. Scand J Work Environ Health. 39(6):618-630. http://dx.doi.org/10.5271/sjweh.3358

Mušák L, Vodicka P, Klimentova G, Soucek P, Hanova M, Mikulkova R, Buchancova J, Vodickova L, Polakova V, Pec M. 2006. Chromosomal damage and polymorphisms of DNA repair genes XRCC1 and XRCC3 in workers exposed to cytostatics. Neuro Endocrinol Lett. 27 Suppl 2:57-60.

Nikula E, Kiviniitty K, Leisti J, Taskinen PJ. 1984. Chromosome aberrations in lymphocytes of nurses handling cytostatic agents. Scand J Work Environ Health. 10(2):71-74. http://dx.doi.org/10.5271/sjweh.2355

Peelen S, Roeleveld N, Heederik D, Kromhout H, De Kort W. 1999. Reproductietoxische effecten bij ziekenhuispersoneel [Toxic effects on reproduction in hospital personnel]. The Hague, Netherlands: Dutch Ministry of Social Affairs and Employment.

Pilger A, Kohler I, Stettner H, Mader RM, Rizovski B, Terkola R, Diem E, Franz-Hainzl E, Konnaris C, Valic E et al. 2000. Long-term monitoring of sister chromatid exchanges and micronucleus frequencies in pharmacy personnel occupationally exposed to cytostatic drugs. Int Arch Occup Environ Health. 73(7):442-448. http://dx.doi.org/10.1007/s004200000164

Pohlová H, Cerna M, Rossner P. 1986. Chromosomal aberrations, SCE and urine mutagenicity in workers occupationally exposed to cytostatic drugs. Mutat Res. 174(3):213-217. http://dx.doi.org/10.1016/0165-7992(86)90154-5

Ratner PA, Spinelli JJ, Beking K, Lorenzi M, Chow Y, Teschke K, Le ND, Gallagher RP, Dimich-Ward H. 2010. Cancer incidence and adverse pregnancy outcome in registered nurses potentially exposed to antineoplastic drugs. BMC Nurs. 9:15. http://dx.doi.org/10.1186/1472$\underline{6955-9-15}$

Rekhadevi PV, Sailaja N, Chandrasekhar M, Mahboob M, Rahman MF, Grover P. 2007. Genotoxicity assessment in oncology nurses handling anti-neoplastic drugs. Mutagenesis. 22(6):395-401. http://dx.doi.org/10.1093/mutage/gem032

Rodríguez-Montero HM, Argote-Pelegrino E, Díaz-Curbelo A, Cuétara-Lugo EB. 2016. Genotoxicity biomarkers for monitoring occupational exposure to antineoplastic drugs. J Pharm Pharmacogn Res. 4(3):122-133.

Rogers B, Emmett EA. 1987. Handling antineoplastic agents: Urine mutagenicity in nurses. Image J Nurs Sch. 19(3):108-113. http://dx.doi.org/10.1111/j.1547-5069.1987.tb00604.x

Rombaldi F, Cassini C, Salvador M, Saffi J, Erdtmann B. 2009. Occupational risk assessment of genotoxicity and oxidative stress in workers handling anti-neoplastic drugs during a working week. Mutagenesis. 24(2):143-148. http://dx.doi.org/10.1093/mutage/gen060

Roth S, Norppa H, Jarventaus H, Kyyronen P, Ahonen M, Lehtomaki J, Sainio H, Sorsa M. 1994. Analysis of chromosomal aberrations, sister-chromatid exchanges and micronuclei in 
peripheral lymphocytes of pharmacists before and after working with cytostatic drugs. Mutat Res. 325(4):157-162. http://dx.doi.org/10.1016/0165-7992(94)90079-5

Rubeš J, Kucharova S, Vozdova M, Musilova P, Zudova Z. 1998. Cytogenetic analysis of peripheral lymphocytes in medical personnel by means of FISH. Mutat Res. 412(3):293-298. http://dx.doi.org/10.1016/S1383-5718(97)00201-5

Sasaki M, Dakeishi M, Hoshi S, Ishii N, Murata K. 2008. Assessment of DNA damage in Japanese nurses handling antineoplastic drugs by the comet assay. J Occup Health. 50(1):7-12. http://dx.doi.org/10.1539/joh.50.7

Saurel-Cubizolles MJ, Job-Spira N, Estryn-Behar M. 1993. Ectopic pregnancy and occupational exposure to antineoplastic drugs. Lancet. 341(8854):1169-1171. http://dx.doi.org/10.1016/0140$\underline{6736(93) 91000-C}$

Schaumburg I, Olsen J. 1989. Time to pregnancy among Danish pharmacy assistants. Scand J Work Environ Health. 15:222-226. http://dx.doi.org/10.5271/sjweh.1859

Schaumburg I, Olsen J. 1990a. Congenital malformations and death among the offspring of Danish pharmacy assistants. Am J Ind Med. 18(5):555-564.

http://dx.doi.org/10.1002/ajim.4700180506

Schaumburg I, Olsen J. 1990b. Risk of spontaneous abortion among Danish pharmacy assistants. Scand J Work Environ Health. 16(3):169-174. http://dx.doi.org/10.5271/sjweh.1797

Schaumburg I, Olsen J. 1991. Birth weight and gestational age among children of Danish pharmacy assistants. J Epidemiol Commun H. 45(1):49-51.

http://dx.doi.org/10.1136/jech.45.1.49

Selevan SG, Lindbohm ML, Hornung RW, Hemminki K. 1985. A study of occupational exposure to antineoplastic drugs and fetal loss in nurses. N Engl J Med. 313(19):1173-1178. http://dx.doi.org/10.1056/NEJM198511073131901

Sessink PJ, Cerna M, Rossner P, Pastorkova A, Bavarova H, Frankova K, Anzion RB, Bos RP. 1994a. Urinary cyclophosphamide excretion and chromosomal aberrations in peripheral blood lymphocytes after occupational exposure to antineoplastic agents. Mutat Res. 309(2):193-199. http://dx.doi.org/10.1016/0027-5107(94)90092-2

Sessink PJ, Verplanke AJ, Herber RF, Bos RP. 1997a. Occupational exposure to antineoplastic agents and parameters for renal dysfunction. Int Arch Occup Environ Health. 69(3):215-218. http://dx.doi.org/10.1007/s004200050139

Shahrasbi AA, Afshar M, Shokraneh F, Monji F, Noroozi M, Ebrahimi-Khojin M, Madani SF, Ahadi-Barzoki M, Rajabi M. 2014. Risks to health professionals from hazardous drugs in Iran: A pilot study of understanding of healthcare team to occupational exposure to cytotoxics. EXCLI Journal. 13:491-501.

Shortridge LA, Lemasters GK, Valanis B, Hertzberg V. 1995. Menstrual cycles in nurses handling antineoplastic drugs. Cancer Nurs. 18(6):439-444. http://dx.doi.org/10.1097/00002820$\underline{199512000-00003}$ 
Skov T, Maarup B, Olsen J, Rorth M, Winthereik H, Lynge E. 1992. Leukaemia and reproductive outcome among nurses handling antineoplastic drugs. Brit J Ind Med. 49(12):855861.

Sorsa M, Pyy L, Salomaa S, Nylund L, Yager JW. 1988. Biological and environmental monitoring of occupational exposure to cyclophosphamide in industry and hospitals. Mutat Res. 204(3):465-479. http://dx.doi.org/10.1016/0165-1218(88)90042-0

Spatari G, Fenga C, Minciullo PL, Di Pasquale G, Cacciola A, Ventura-Spagnolo E, Gangemi S. 2005. Modification of interleukin-15 serum levels in workers exposed to chemotherapeutic agents. Mediators Inflamm. 2005(1):60-62. http://dx.doi.org/10.1155/MI.2005.60

Stücker I, Caillard JF, Collin R, Gout M, Poyen D, Hemon D. 1990. Risk of spontaneous abortion among nurses handling antineoplastic drugs. Scand J Work Environ Health. 16(2):102107. http://dx.doi.org/10.5271/sjweh.1811

Stücker I, Hirsch A, Doloy T, Bastie-Sigeac I, Hemon D. 1986. Urine mutagenicity, chromosomal abnormalities and sister chromatid exchanges in lymphocytes of nurses handling cytostatic drugs. Int Arch Occup Environ Health. 57(3):195-205.

http://dx.doi.org/10.1007/BF00405788

Stücker I, Mandereau L, Hemon D. 1993. Relationship between birthweight and occupational exposure to cytostatic drugs during or before pregnancy. Scand J Work Environ Health. 19(3):148-153. http://dx.doi.org/10.5271/sjweh.1488

Taskinen H, Kyyronen P, Hemminki K, Hoikkala M, Lajunen K, Lindbohm ML. 1994. Laboratory work and pregnancy outcome. J Occup Environ Med. 36(3):311-319. http://dx.doi.org/10.1097/00043764-199403000-00008

Taskinen H, Lindbohm ML, Hemminki K. 1986. Spontaneous abortions among women working in the pharmaceutical-industry. Brit J Ind Med. 43(3):199-205.

Testa A, Giachelia M, Palma S, Appolloni M, Padua L, Tranfo G, Spagnoli M, Tirindelli D, Cozzi R. 2007. Occupational exposure to antineoplastic agents induces a high level of chromosome damage. Lack of an effect of GST polymorphisms. Toxicol Appl Pharmacol. 223(1):46-55. http://dx.doi.org/10.1016/j.taap.2007.05.006

Thiringer G, Granung G, Holmen A, Hogstedt B, Jarvholm B, Jonsson D, Persson L, Wahlstrom J, Westin J. 1991. Comparison of methods for the biomonitoring of nurses handling antitumor drugs. Scand J Work Environ Health. 17(2):133-138. http://dx.doi.org/10.5271/sjweh.1724

Thulin H, Sundberg E, Hansson K, Cole J, Hartley-Asp B. 1995. Occupational exposure to nornitrogen mustard: Chemical and biological monitoring. Toxicol Ind Health. 11(1):89-97. http://dx.doi.org/10.1177/074823379501100108

Tigha Bouaziz N, Tourab D, Nezzal AM. 2016. Study of cardiovascular morbidity in nurses exposed to cytostatic drugs: Multivaried approach analysis. Ann Cardiol Angeiol. 65(3):179-184. http://dx.doi.org/10.1016/j.ancard.2016.04.014 
Ündeğer U, Basaran N, Kars A, Guc D. 1999. Assessment of DNA damage in nurses handling antineoplastic drugs by the alkaline COMET assay. Mutat Res. 439(2):277-285. http://dx.doi.org/10.1016/S1383-5718(99)00002-9

Ursini CL, Cavallo D, Colombi A, Giglio M, Marinaccio A, Iavicoli S. 2006. Evaluation of early DNA damage in healthcare workers handling antineoplastic drugs. Int Arch Occup Environ Health. 80(2):134-140. http://dx.doi.org/10.1007/s00420-006-0111-X

Valanis B, Vollmer W, Labuhn K, Glass A. 1997. Occupational exposure to antineoplastic agents and self-reported infertility among nurses and pharmacists. J Occup Environ Med. 39(6):574-580. http://dx.doi.org/10.1097/00043764-199706000-00013

Valanis B, Vollmer WM, Steele P. 1999. Occupational exposure to antineoplastic agents: Selfreported miscarriages and stillbirths among nurses and pharmacists. J Occup Environ Med. 41(8):632-638. http://dx.doi.org/10.1097/00043764-199908000-00004

Valanis BG, Hertzberg V, Shortridge L. 1987. Antineoplastic drugs. Handle with care. AAOHN J. 35(11):487-492.

Valanis BG, Vollmer WM, Labuhn KT, Glass AG. 1993. Acute symptoms associated with antineoplastic drug handling among nurses. Cancer Nurs. 16(4):288-295. http://dx.doi.org/10.1097/00002820-199308000-00005

Valanis BG, Vollmer WM, Labuhn KT, Glass AG. 1993. Association of antineoplastic drug handling with acute adverse effects in pharmacy personnel. Am J Hosp Pharm. 50(3):455-462.

Villarini M, Dominici L, Piccinini R, Fatigoni C, Ambrogi M, Curti G, Morucci P, Muzi G, Monarca S, Moretti M. 2011. Assessment of primary, oxidative and excision repaired DNA damage in hospital personnel handling antineoplastic drugs. Mutagenesis. 26(3):359-369. http://dx.doi.org/10.1093/mutage/geq102

Walusiak J, Wagrowska-Koski E, Palczynski C. 2003. [Health effects of occupational exposure to cytostatics in medicinal personnel in the light of compulsory prophylactics: a cross-sectional study]. Med Pr. 54(3):229-236.

Yager JW, Sorsa M, Selvin S. 1988. Micronuclei in cytokinesis-blocked lymphocytes as an index of occupational exposure to alkylating cytostatic drugs. IARC Sci Publ. (89):213-216.

Yang D, Xu S, Wang J. 2002a. [The study of DNA damage of peripheral lymphocytes in the nurses occupationally exposed to anticancer drugs]. Zhonghua Lao Dong Wei Sheng Zhi Ye Bing Za Zhi. 20(3):197-199.

Yang DP, Xu SJ, Wang JX. 2002b. Study on chromosome damage among nurses occupationally exposed to antineoplastic drugs in an oncology department. Biomed Environ Sci. 15(3):268-270.

Yin H, Xu XQ, Lin LY. 2010. [DNA damage of antineoplastic drugs exposure on nurses]. Zhonghua Lao Dong Wei Sheng Zhi Ye Bing Za Zhi. 28(3):218-219.

Yoshida J, Kosaka H, Tomioka K, Kumagai S. 2006. Genotoxic risks to nurses from contamination of the work environment with antineoplastic drugs in Japan. J Occup Health. 48(6):517-522. http://dx.doi.org/10.1539/joh.48.517 
Zhang X, Zheng Q, Lv Y, An M, Zhang Y, Wei Y, Feng W. 2016. Evaluation of adverse health risks associated with antineoplastic drug exposure in nurses at two Chinese hospitals: The effects of implementing a pharmacy intravenous admixture service. Am J Ind Med. 59(4):264-273. http://dx.doi.org/10.1002/ajim.22553 


\section{Appendix C. Risk-of-bias Assessment for All Included Studies}

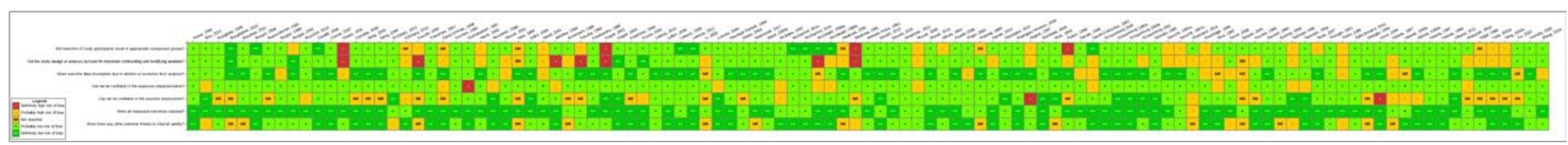

Figure C-1. Risk-of-bias Heat Map for Included Studies Reporting on Health Outcomes

Interactive figure and additional study details in HAWC (NTP, 2015a). Fransman 2007 refers to Fransman et al. (2007c). 


\section{Appendix D. Data Figures}

\section{Figures}

Figure D-1. Spontaneous Abortion Associated with Occupational Exposure to Cancer Chemotherapy Agents Reported as (A) Odds Ratio or (B) Percent of

Pregnancies Affected........................................................................................ D-3

Figure D-2. Risk-of-bias Bar Graph for Studies Reporting Spontaneous Abortions ................ D-4

Figure D-3. Risk-of-bias Heat Map for Studies Reporting Spontaneous Abortions ................. D-4

Figure D-4. Risk-of-bias Bar Graph for All Included Studies Reporting on Genetic

Toxicity Outcomes of Structural Chromosomal Aberrations, Micronucleus

Induction, and DNA Damage Measured by the Comet Assay............................... D-5

Figure D-5. Risk-of-bias Heat Map for All Included Studies Reporting on Genetic

Toxicity Outcomes of Structural Chromosomal Aberrations, Micronucleus

Induction, and DNA Damage Measured by the Comet Assay............................. D-5

Figure D-6. Percent of Lymphocytes with Structural Chromosomal Aberrations

(Excluding Gaps) Associated with Occupational Exposure to Cancer

Chemotherapy Agents Presented as (A) Mean Values or (B) Adjusted Odds

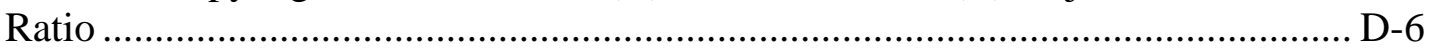

Figure D-7. Number of Chromosomal Aberrations (Excluding Gaps) per 100

Lymphocytes Scored Associated with Occupational Exposure to Cancer

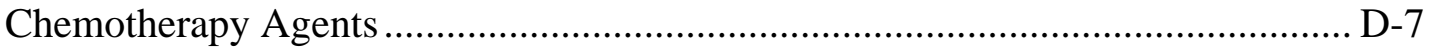

Figure D-8. Risk-of-bias Bar Graph for All Included Studies Reporting on Structural

Chromosomal Aberrations ............................................................................ D-8

Figure D-9. Risk-of-bias Heat Map for All Included Studies Reporting on Structural

Chromosomal Aberrations

Figure D-10. Number of Cells with Micronuclei/1,000 Cells Scored Associated with

Occupational Exposure to Cancer Chemotherapy Agents for (A)

Binucleated Lymphocytes or (B) Buccal Cells....

Figure D-11. Number of Micronuclei/1,000 Cells Scored Associated with Occupational Exposure to Cancer Chemotherapy Agents for Binucleated Lymphocytes

Figure D-12. Number of Micronuclei/1,000 Cells Scored Associated with Occupational Exposure to Cancer Chemotherapy Agents for Buccal Cells D-11

Figure D-13. Risk-of-bias Bar Graph for Included Studies Reporting on Micronucleus Induction

Figure D-14. Risk-of-bias Heat Map for Included Studies Reporting on Micronucleus Induction

Figure D-15. Percent of Tail DNA Measured by the Comet Assay Associated with Occupational Exposure to Cancer Chemotherapy Agents for (A) Studies Reporting Values in the Expected Range and (B) Studies Reporting Higher than Expected Values.

Figure D-16. Tail Length $(\mu \mathrm{m})$ Measured by the Comet Assay Associated with Occupational Exposure to Cancer Chemotherapy Agents

Figure D-17. Tail Moment Measured by the Comet Assay Associated with Occupational Exposure to Cancer Chemotherapy Agents for (A) Studies in the Expected Range and (B) Studies in Higher than the Expected Range ..... 
Figure D-18. DNA Damage Index Measured by the Comet Assay Associated with Occupational Exposure to Cancer Chemotherapy Agents Reported as (A) Mean Comet Score or (B) Mean Number of Cells ..... D-16

Figure D-19. Risk-of-bias Bar Graph for Included Studies Reporting DNA Damage Measured by the Comet Assay.

Figure D-20. Risk-of-bias Heat Map for Included Studies Reporting DNA Damage Measured by the Comet Assay.... 
A

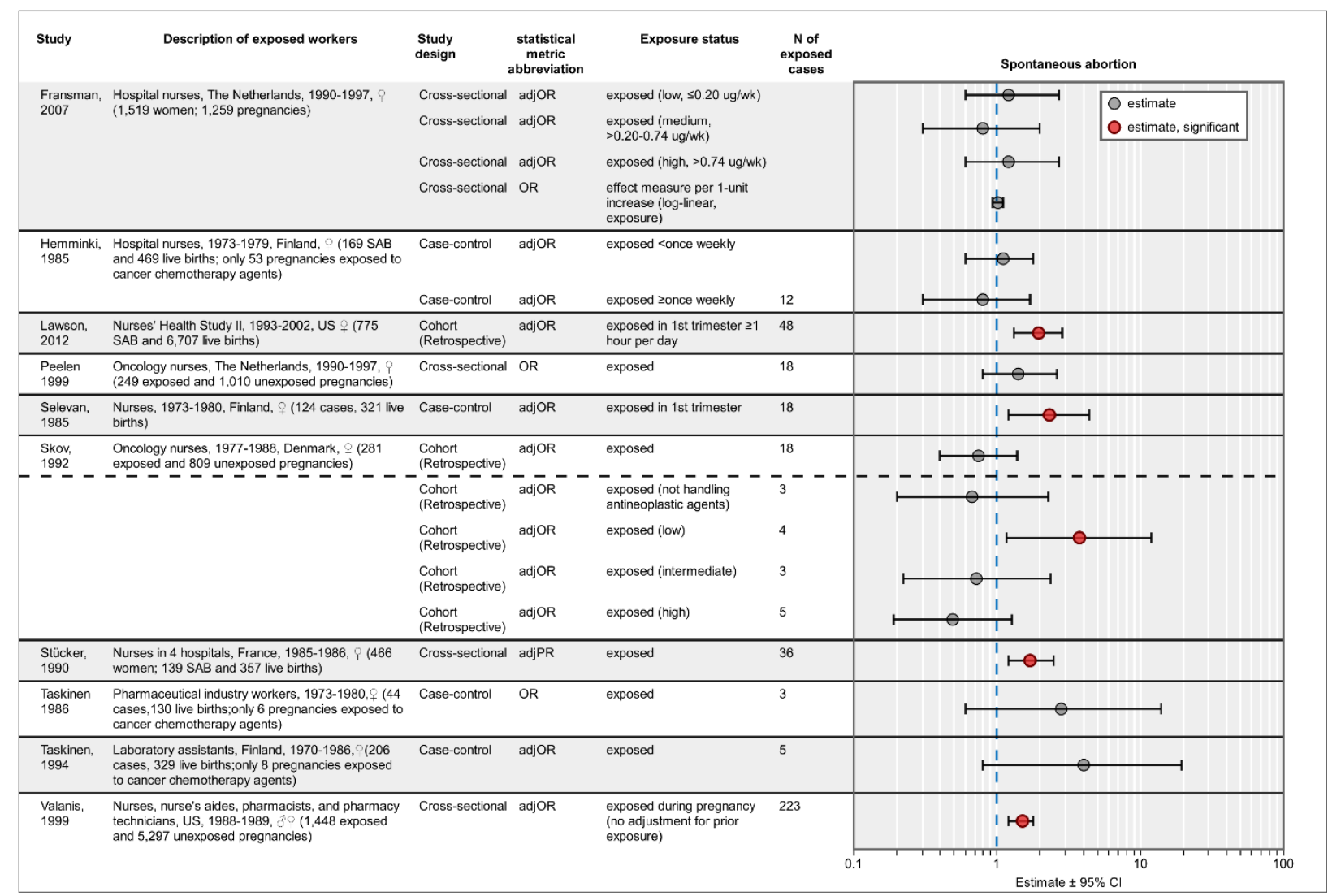

B

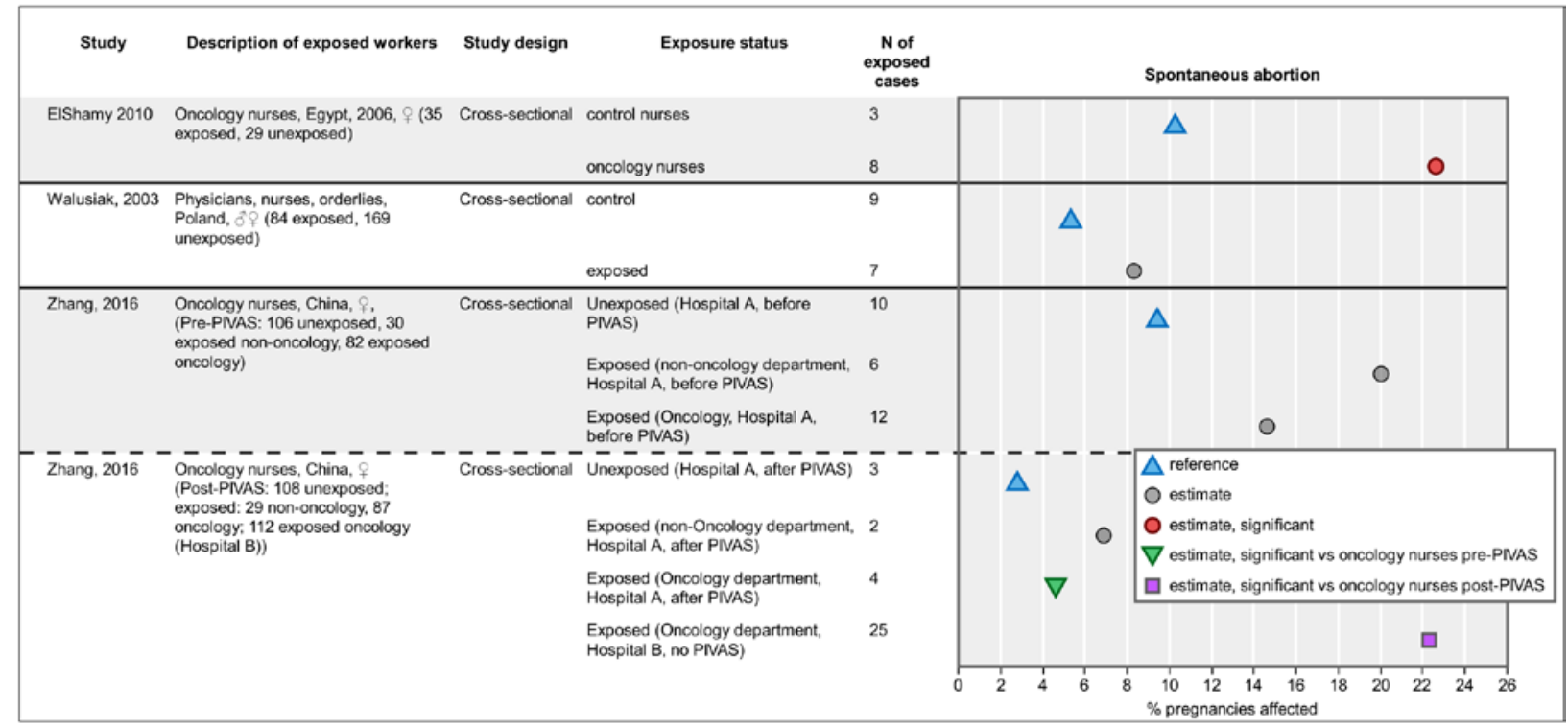

Figure D-1. Spontaneous Abortion Associated with Occupational Exposure to Cancer Chemotherapy Agents Reported as (A) Odds Ratio or (B) Percent of Pregnancies Affected

Interactive figure and additional study details in HAWC (NTP, 2015a). Reference line is indicated as a vertical, blue dotted line in A. Fransman 2007 refers to Fransman et al. (2007c). Number of cases for Valanis et al. (1999) include the spouses of exposed male employees; however, the data graphed are pregnancy outcomes of exposed female employees only. Three studies are not 
graphed: McDonald et al. (1988b) reported that oncology nurses handling antineoplastic drugs had similar rates of spontaneous abortion compared to control subjects (observed versus expected ratio $=0.97$ ), Medková (1991b) reported a higher number of early spontaneous abortions in exposed medical personnel (8 of 74 pregnancies) compared to unexposed spouses of medical personnel (0 of 11 pregnancies), and Schaumburg and Olsen (1990b) observed no spontaneous abortions among eight pregnancies of exposed pharmacy assistants.

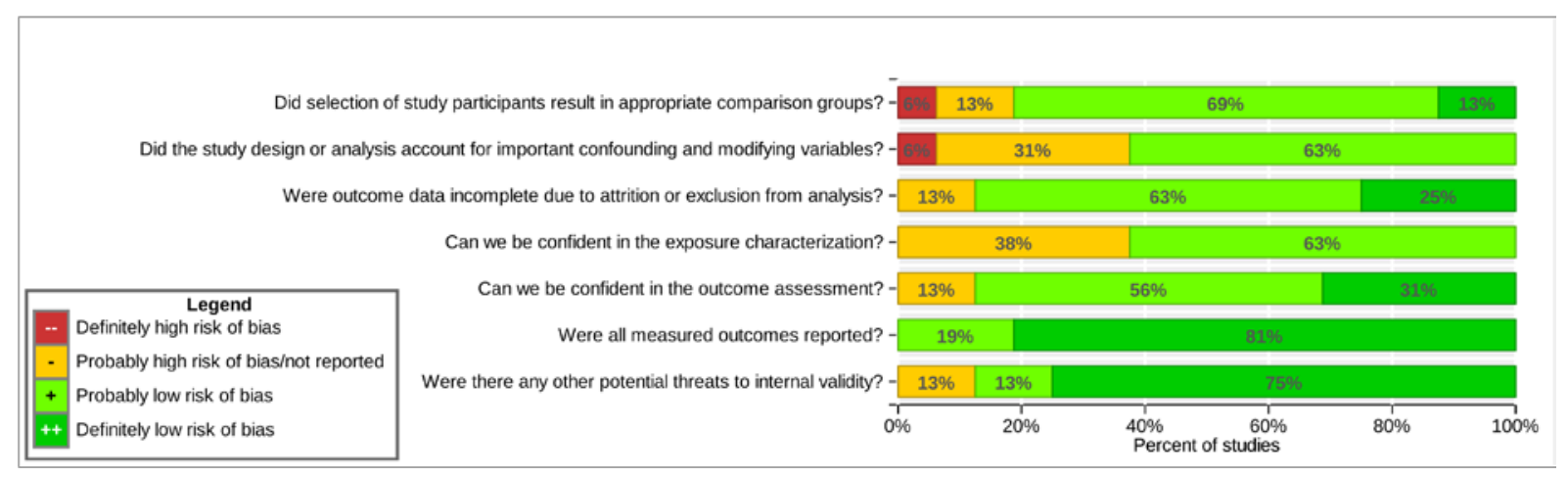

Figure D-2. Risk-of-bias Bar Graph for Studies Reporting Spontaneous Abortions

Interactive figure and additional study details in HAWC (NTP, 2015a).

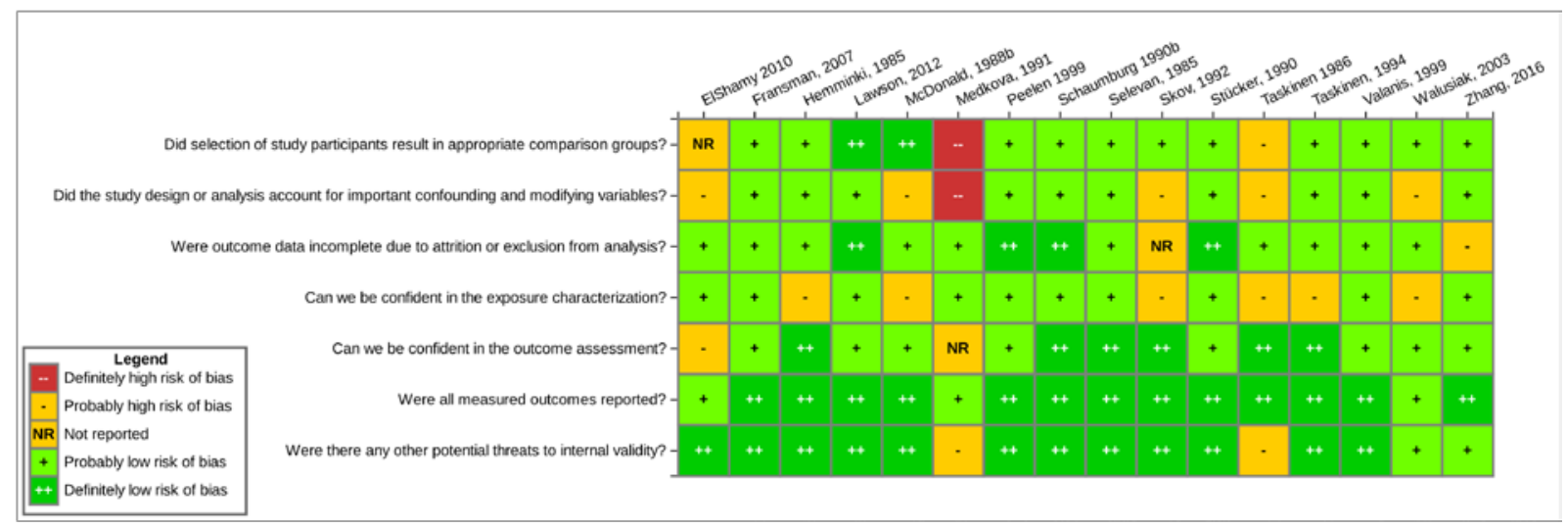

Figure D-3. Risk-of-bias Heat Map for Studies Reporting Spontaneous Abortions

Interactive figure and additional study details in HAWC (NTP, 2015a). Fransman 2007 refers to Fransman et al. (2007c). 


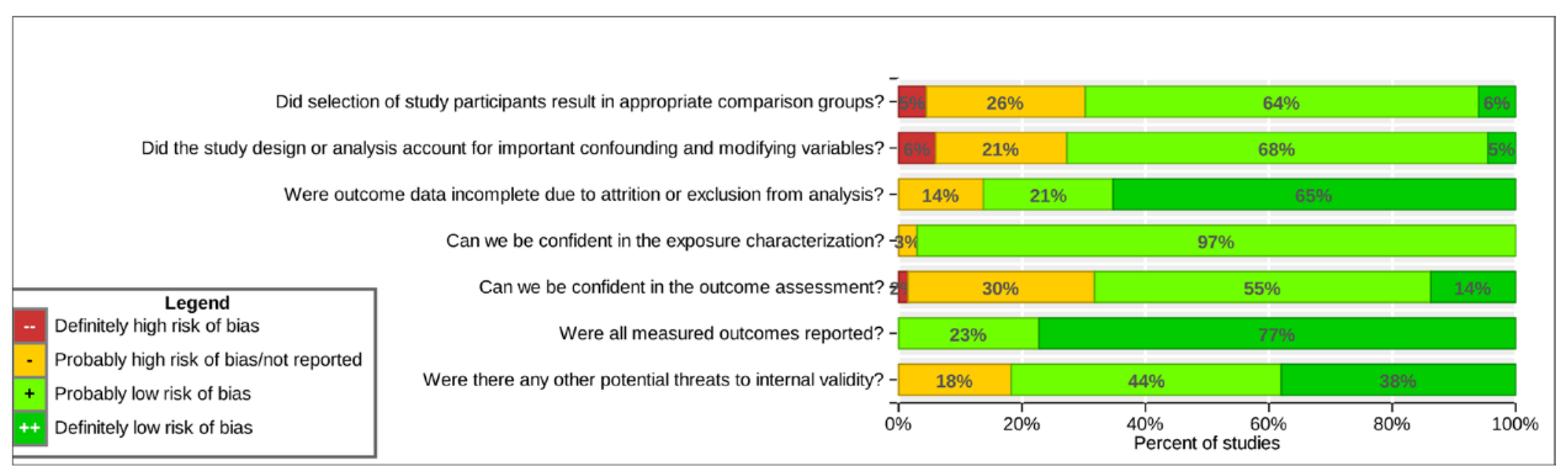

Figure D-4. Risk-of-bias Bar Graph for All Included Studies Reporting on Genetic Toxicity Outcomes of Structural Chromosomal Aberrations, Micronucleus Induction, and DNA Damage Measured by the Comet Assay

Interactive figure and additional study details in HAWC (NTP, 2015a).

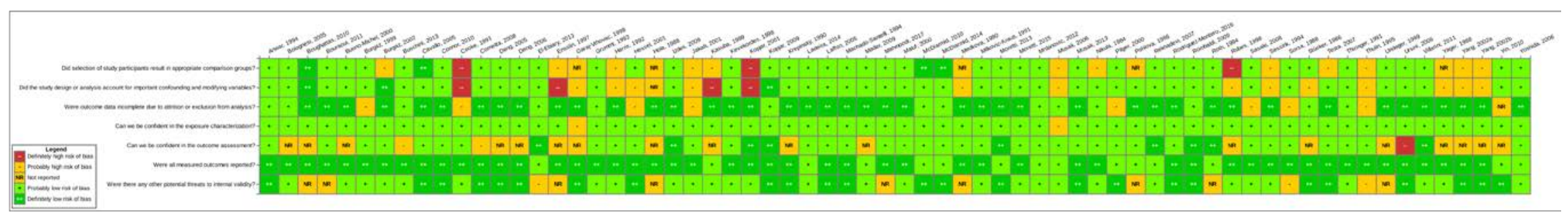

Figure D-5. Risk-of-bias Heat Map for All Included Studies Reporting on Genetic Toxicity Outcomes of Structural Chromosomal Aberrations, Micronucleus Induction, and DNA Damage Measured by the Comet Assay

Interactive figure and additional study details in HAWC (NTP, 2015a). 


\section{A}

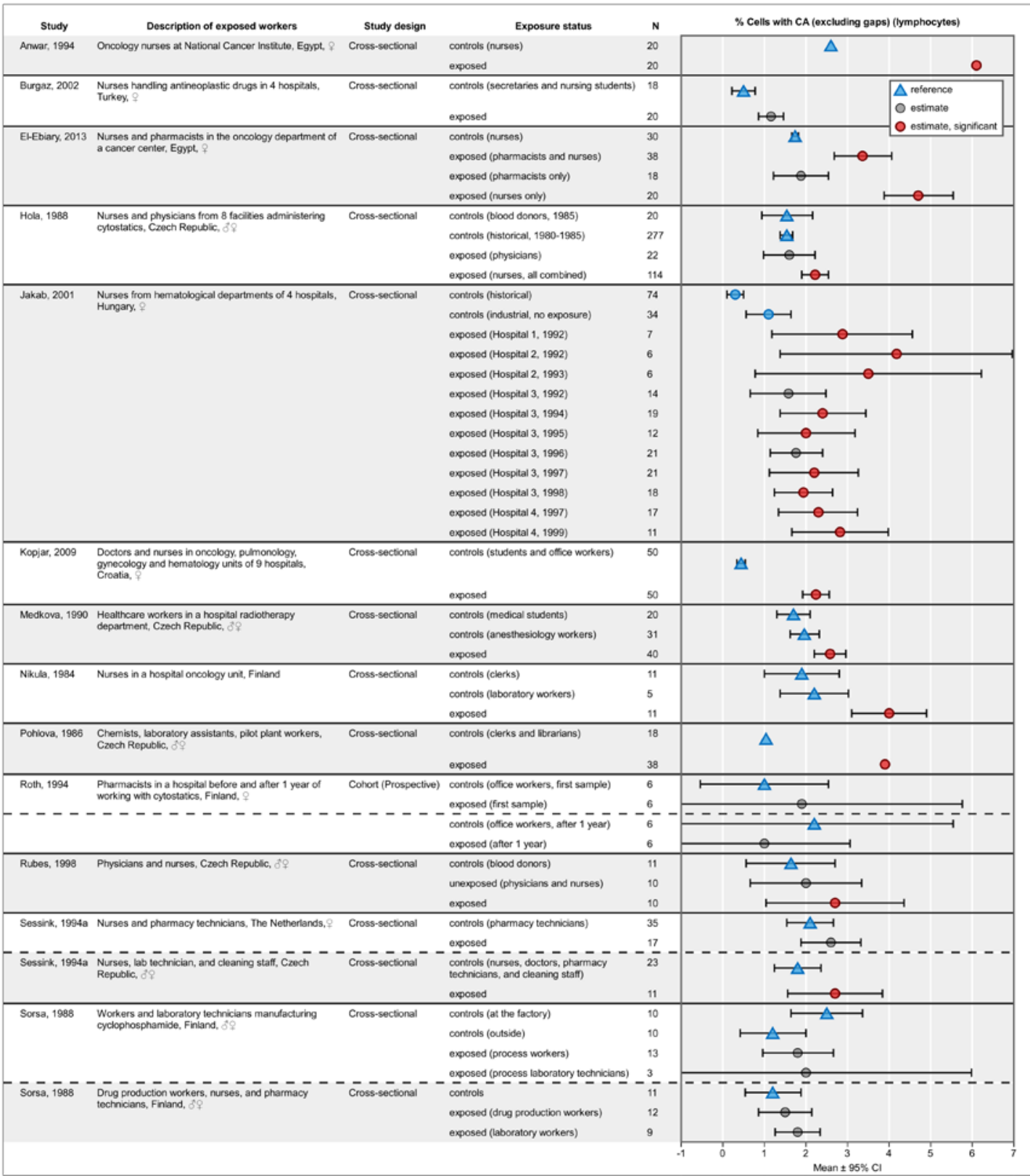

B

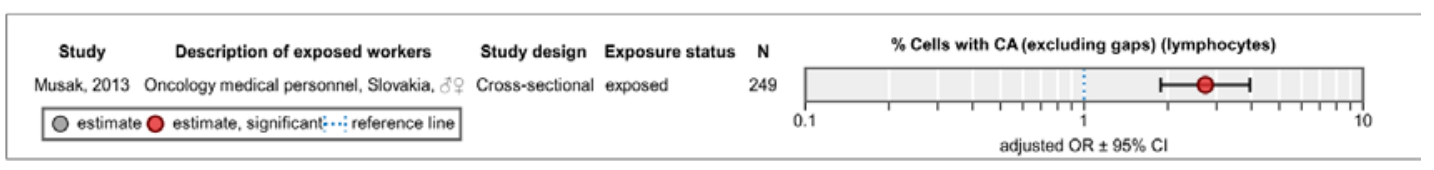

Figure D-6. Percent of Lymphocytes with Structural Chromosomal Aberrations (Excluding Gaps) Associated with Occupational Exposure to Cancer Chemotherapy Agents Presented as (A) Mean Values or (B) Adjusted Odds Ratio

Interactive figures and additional study details in HAWC (NTP, 2015a). Controls refer to the unexposed, reference population. In part B, reference line indicated by blue, dotted line. 


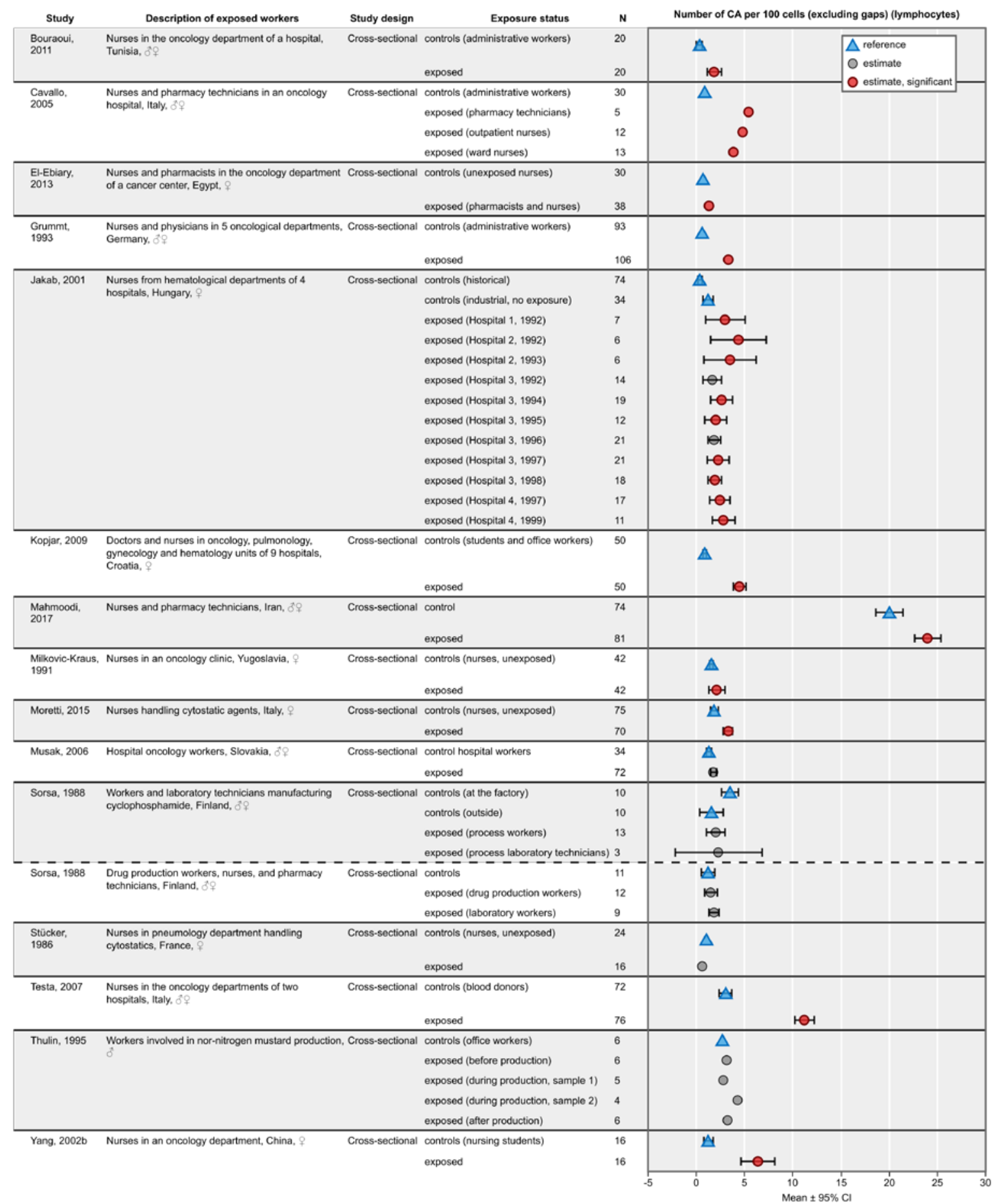

Figure D-7. Number of Chromosomal Aberrations (Excluding Gaps) per 100 Lymphocytes Scored Associated with Occupational Exposure to Cancer Chemotherapy Agents

Interactive figure and additional study details in HAWC (NTP, 2015a). Controls refer to the unexposed, reference population. Two studies reporting no difference between exposed and unexposed subjects do not appear on the figure because the authors did not report the data values in the publication (Krepinsky et al. 1990) or the reported data were log-transformed and thus could not be included on the same graph with remainder of the data (Cooke et al. 1991). 


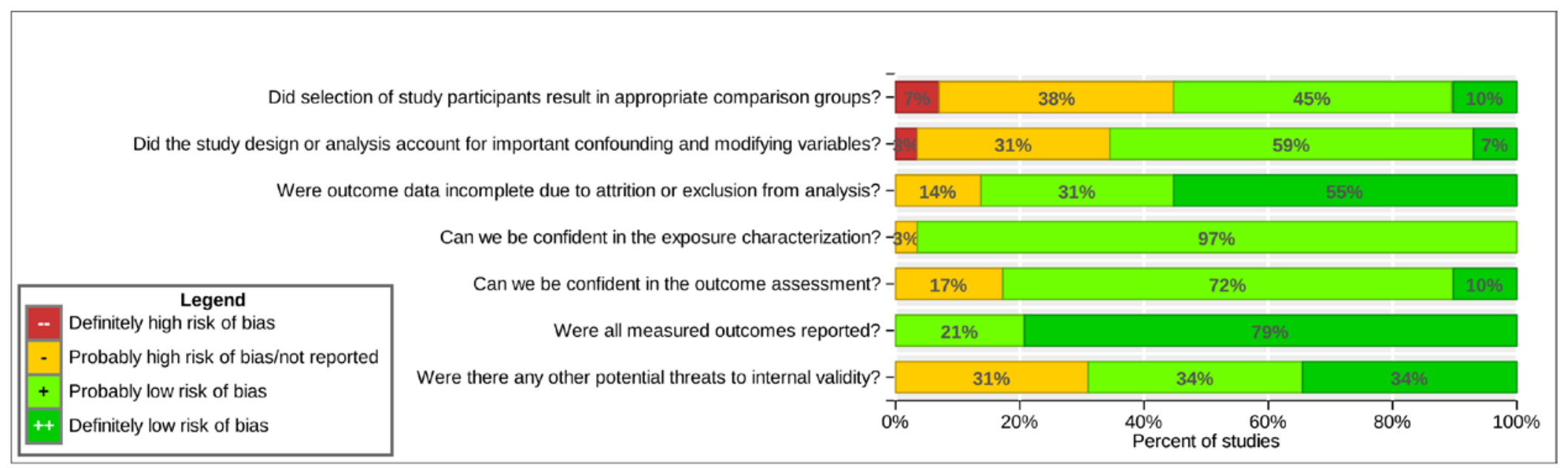

Figure D-8. Risk-of-bias Bar Graph for All Included Studies Reporting on Structural Chromosomal Aberrations

Interactive figure and additional study details in HAWC (NTP, 2015a).

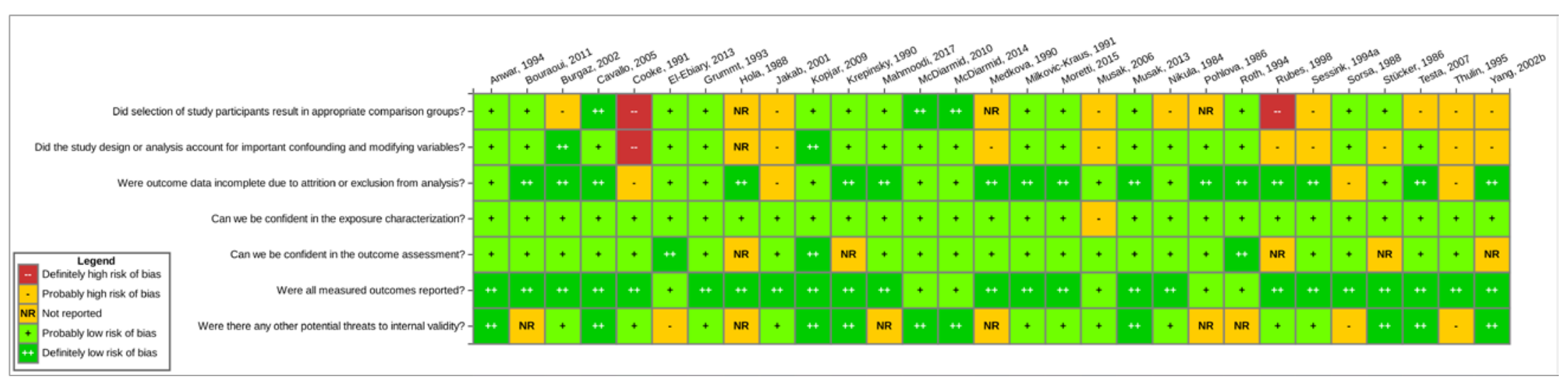

Figure D-9. Risk-of-bias Heat Map for All Included Studies Reporting on Structural Chromosomal Aberrations

Interactive figure and additional study details in HAWC (NTP, 2015a). 


\section{A}

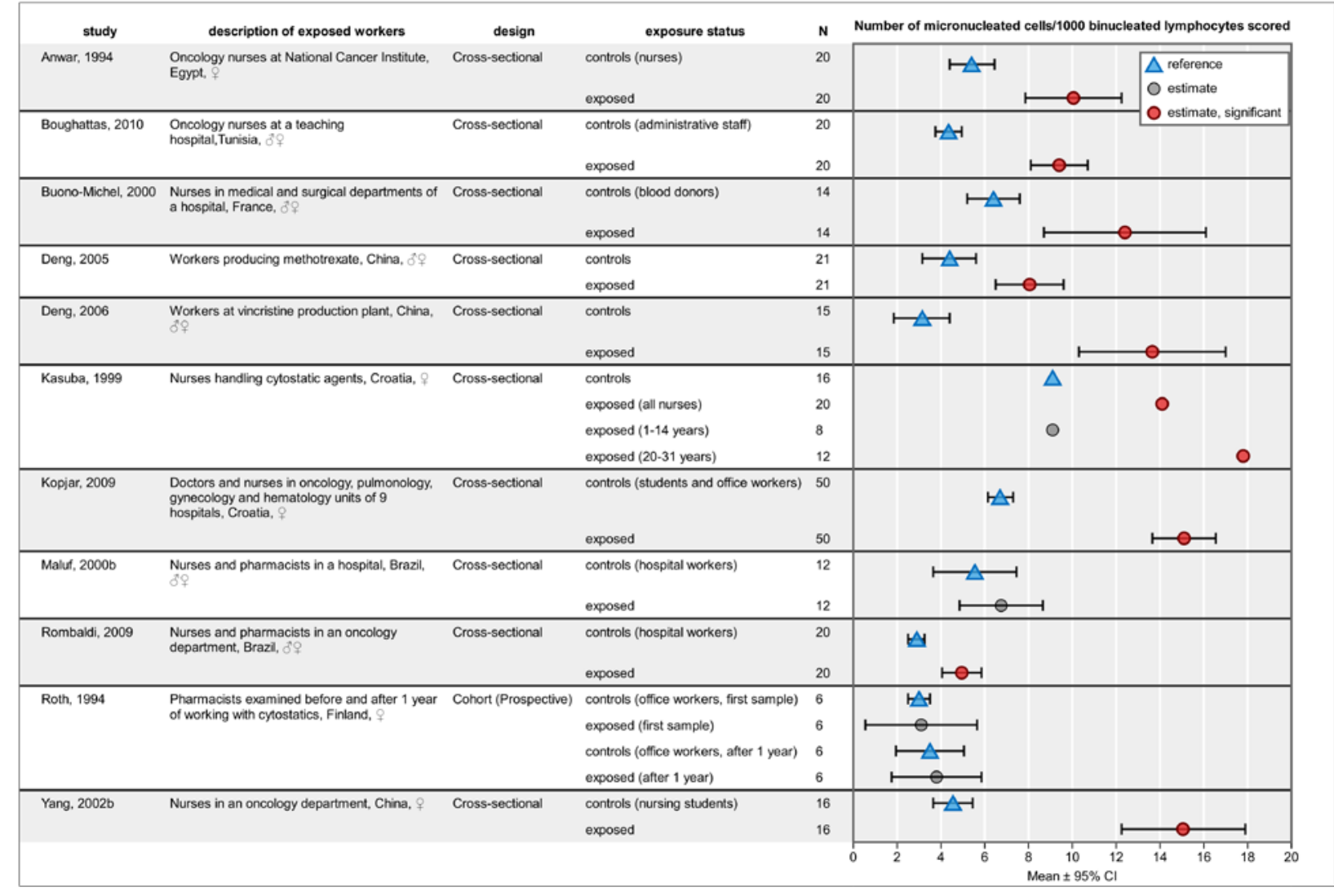

B

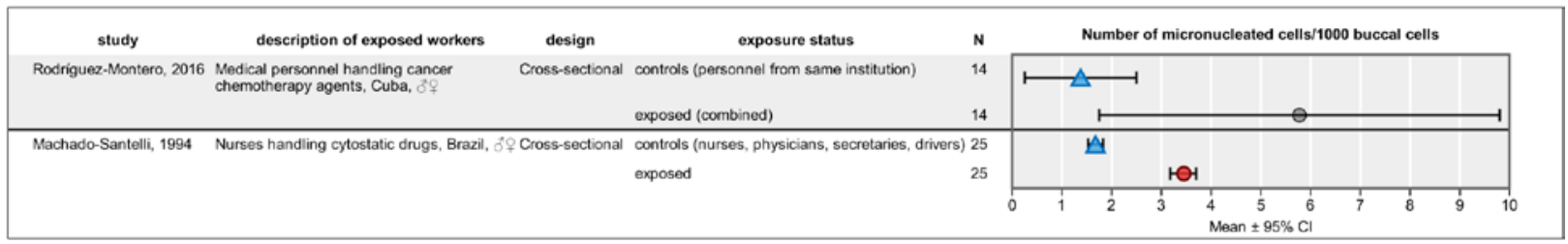

Figure D-10. Number of Cells with Micronuclei/1,000 Cells Scored Associated with Occupational Exposure to Cancer Chemotherapy Agents for (A) Binucleated Lymphocytes or (B) Buccal Cells

Interactive figure and additional study details in HAWC (NTP, 2015a). Controls refer to the unexposed, reference population. 
Systematic Review of Occupational Exposure to Cancer Chemotherapy Agents

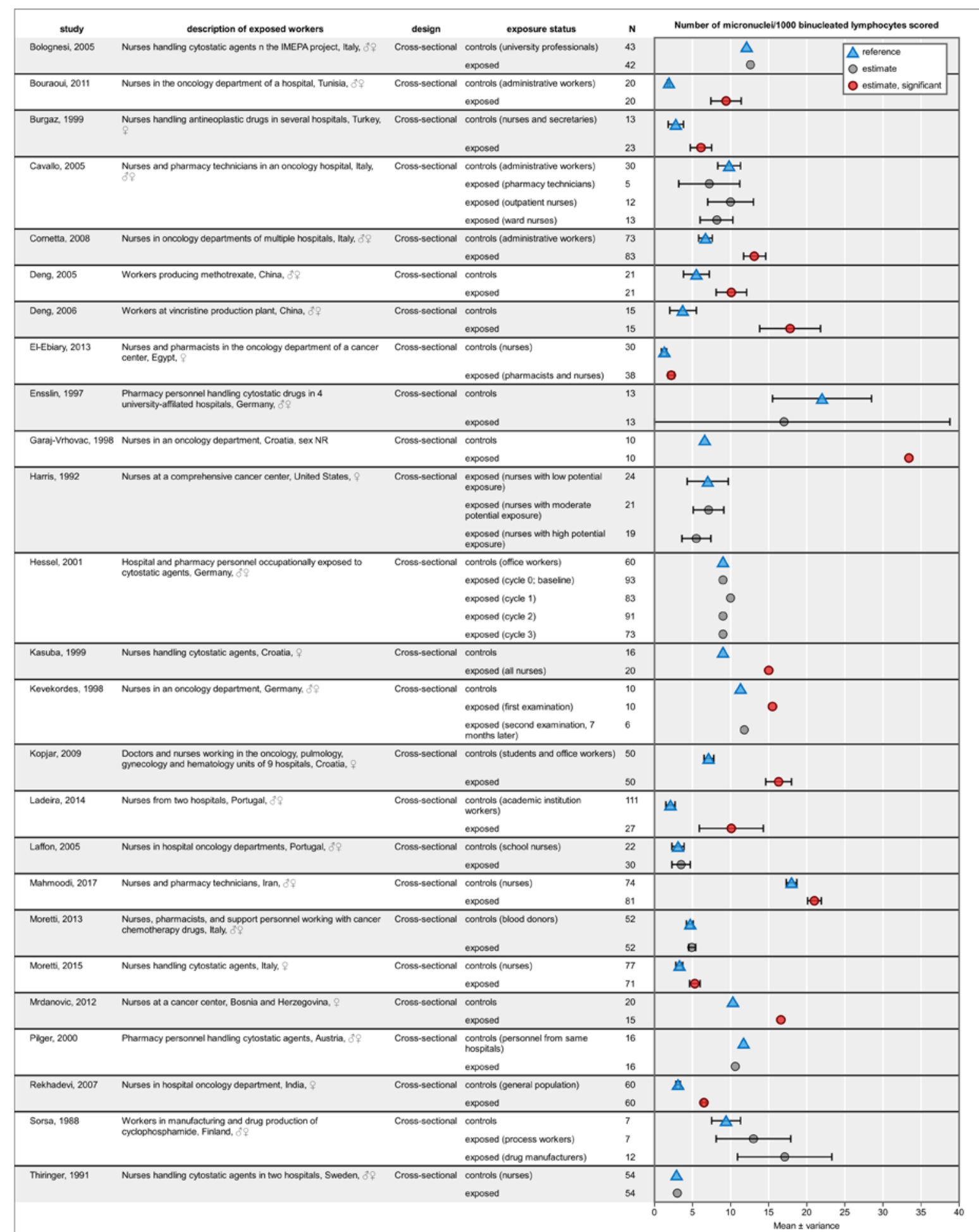

Figure D-11. Number of Micronuclei/1,000 Cells Scored Associated with Occupational Exposure to Cancer Chemotherapy Agents for Binucleated Lymphocytes

Interactive figure and additional study details in HAWC (NTP, 2015a). Controls refer to the unexposed, reference population. An additional study of production workers handling cyclophosphamide reported a significantly $(\mathrm{p}<0.05)$ higher mean logtransformed number of MN/1,000 cells scored in an ANOVA adjusting for age differences (data not shown) (Yager et al. 1988). 
Systematic Review of Occupational Exposure to Cancer Chemotherapy Agents

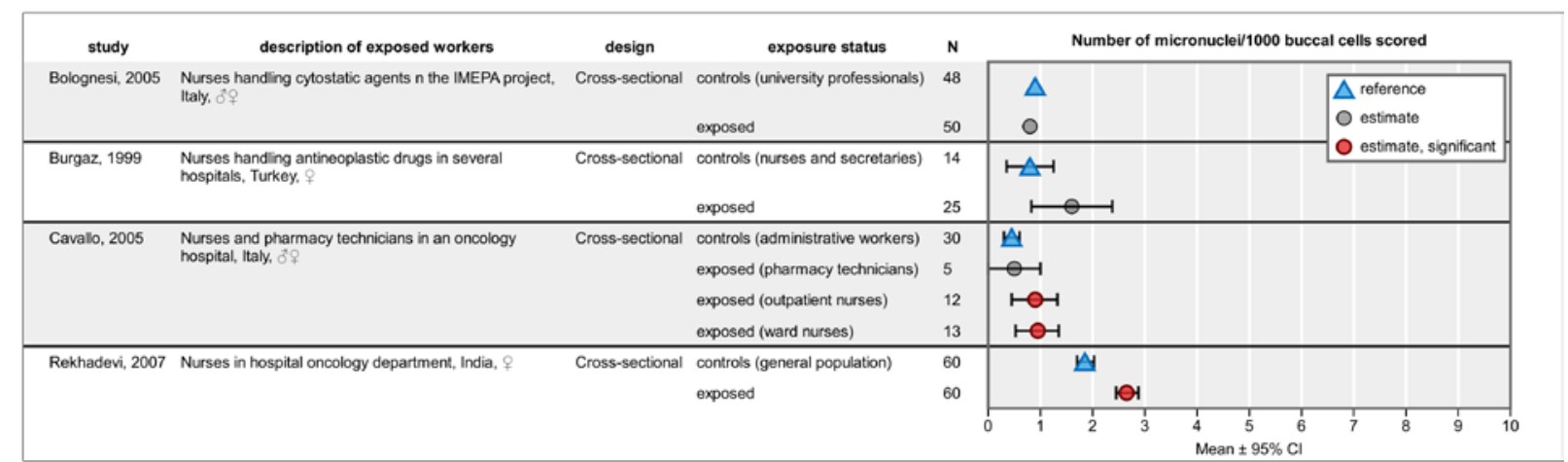

Figure D-12. Number of Micronuclei/1,000 Cells Scored Associated with Occupational Exposure to Cancer Chemotherapy Agents for Buccal Cells

Interactive figure and additional study details in HAWC (NTP, 2015a). Controls refer to the unexposed, reference population. 


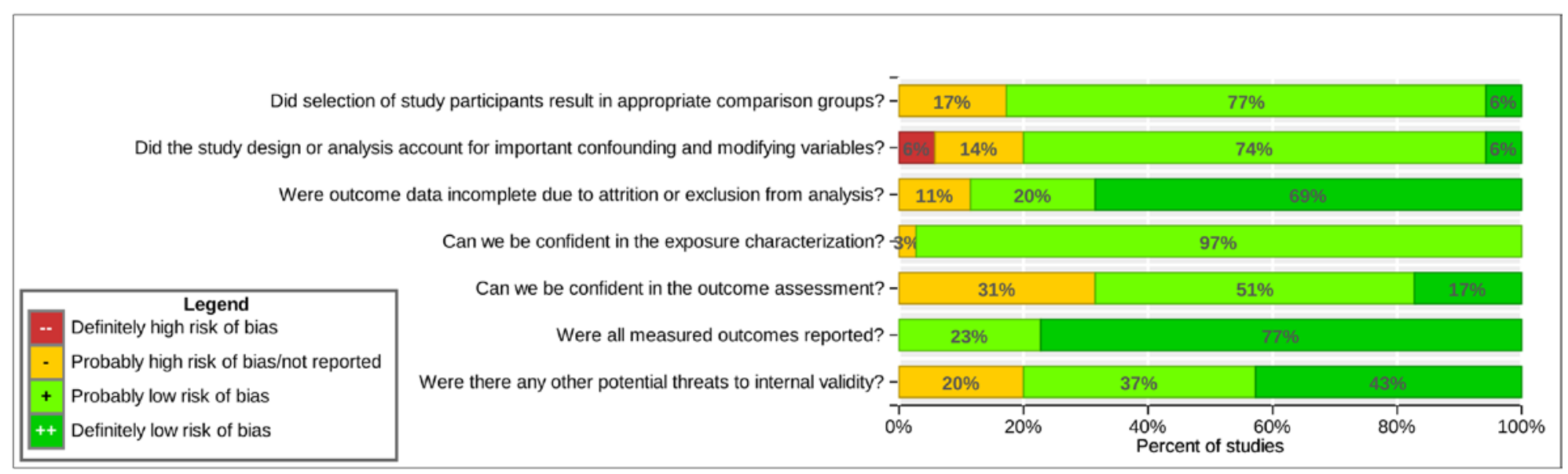

Figure D-13. Risk-of-bias Bar Graph for Included Studies Reporting on Micronucleus Induction

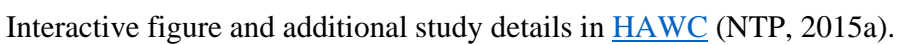

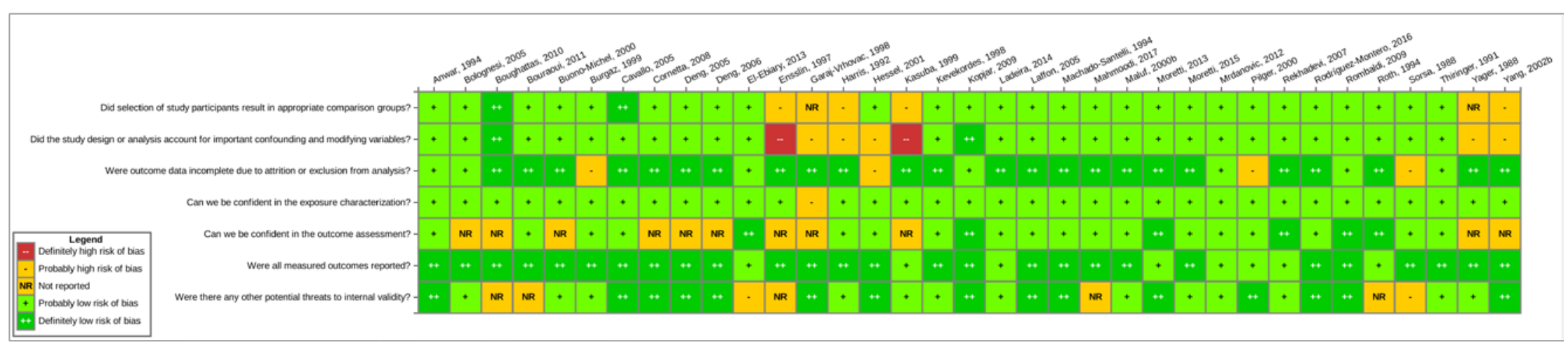

Figure D-14. Risk-of-bias Heat Map for Included Studies Reporting on Micronucleus Induction

Interactive figure and additional study details in $\underline{\text { HAWC }}$ (NTP, 2015a). 


\section{A}

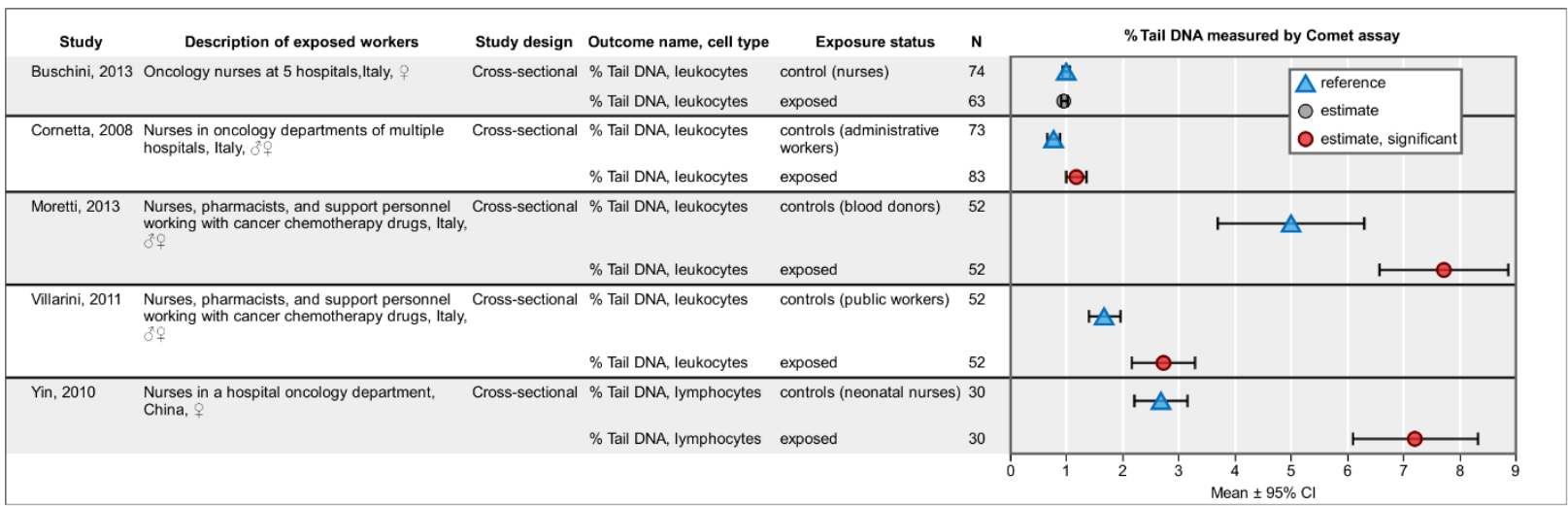

B

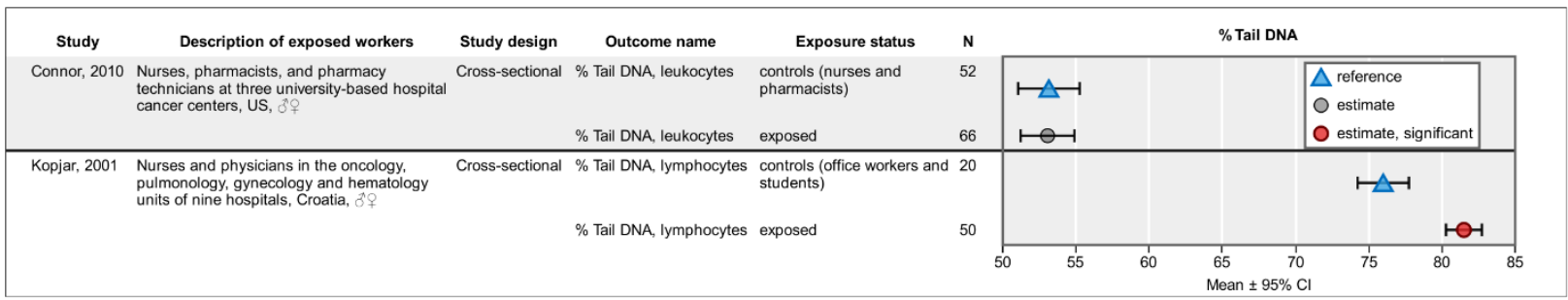

Figure D-15. Percent of Tail DNA Measured by the Comet Assay Associated with Occupational Exposure to Cancer Chemotherapy Agents for (A) Studies Reporting Values in the Expected Range and (B) Studies Reporting Higher than Expected Values

Interactive figure and additional study details in HAWC (NTP, 2015a). Controls refer to the unexposed, reference population. 
Systematic Review of Occupational Exposure to Cancer Chemotherapy Agents

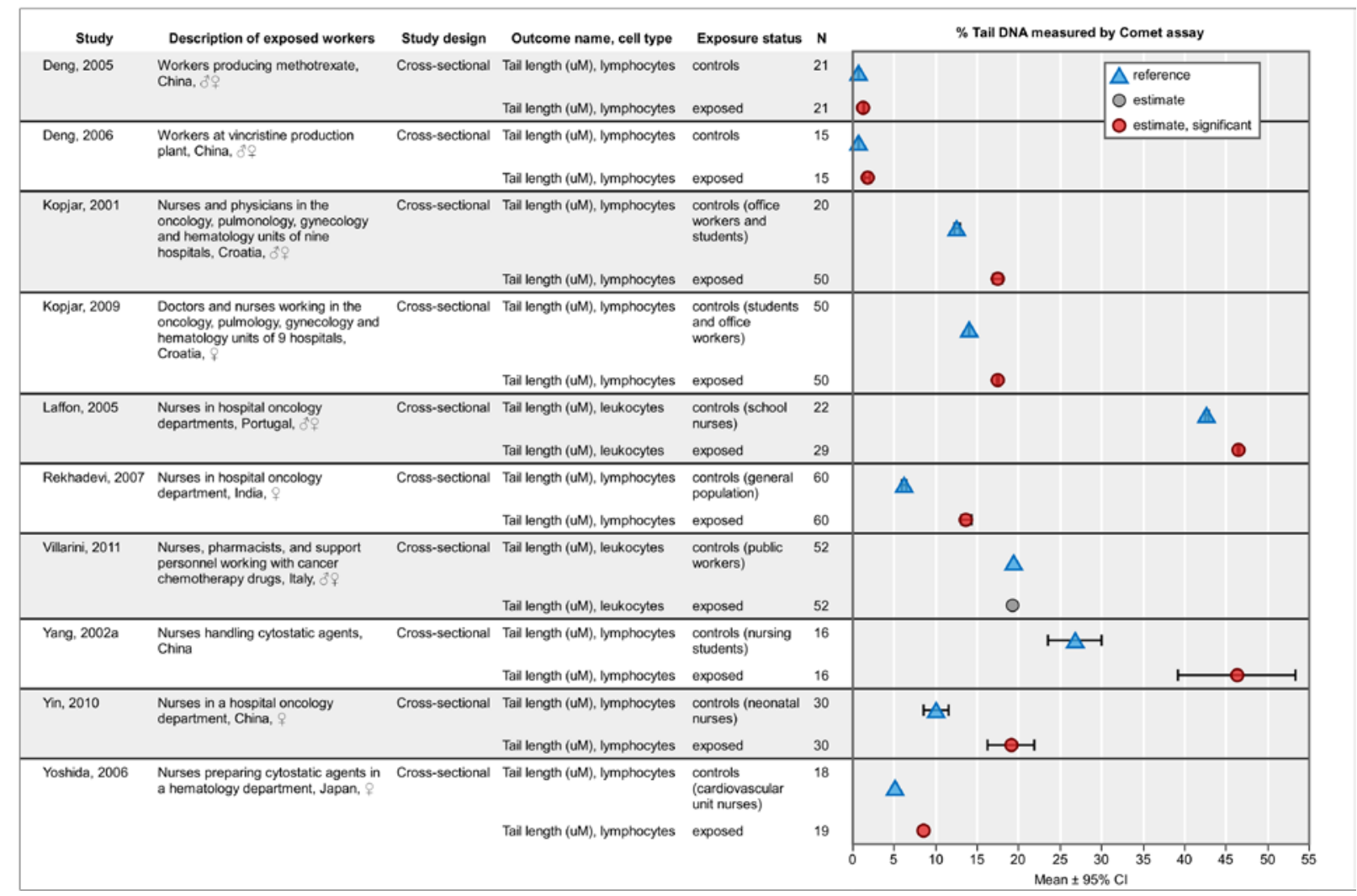

Figure D-16. Tail Length $(\mu \mathrm{m})$ Measured by the Comet Assay Associated with Occupational Exposure to Cancer Chemotherapy Agents

Interactive figure and additional study details in HAWC (NTP, 2015a). Controls refer to the unexposed, reference population. An additional study reported a greater log-transformed mean tail length $(\mu \mathrm{m})$ in exposed nurses than controls following adjustment for age, smoking, coffee-drinking habits, hemoglobin, ionizing radiation, and hospital (data not graphed) (Sasaki et al. 2008). 
A

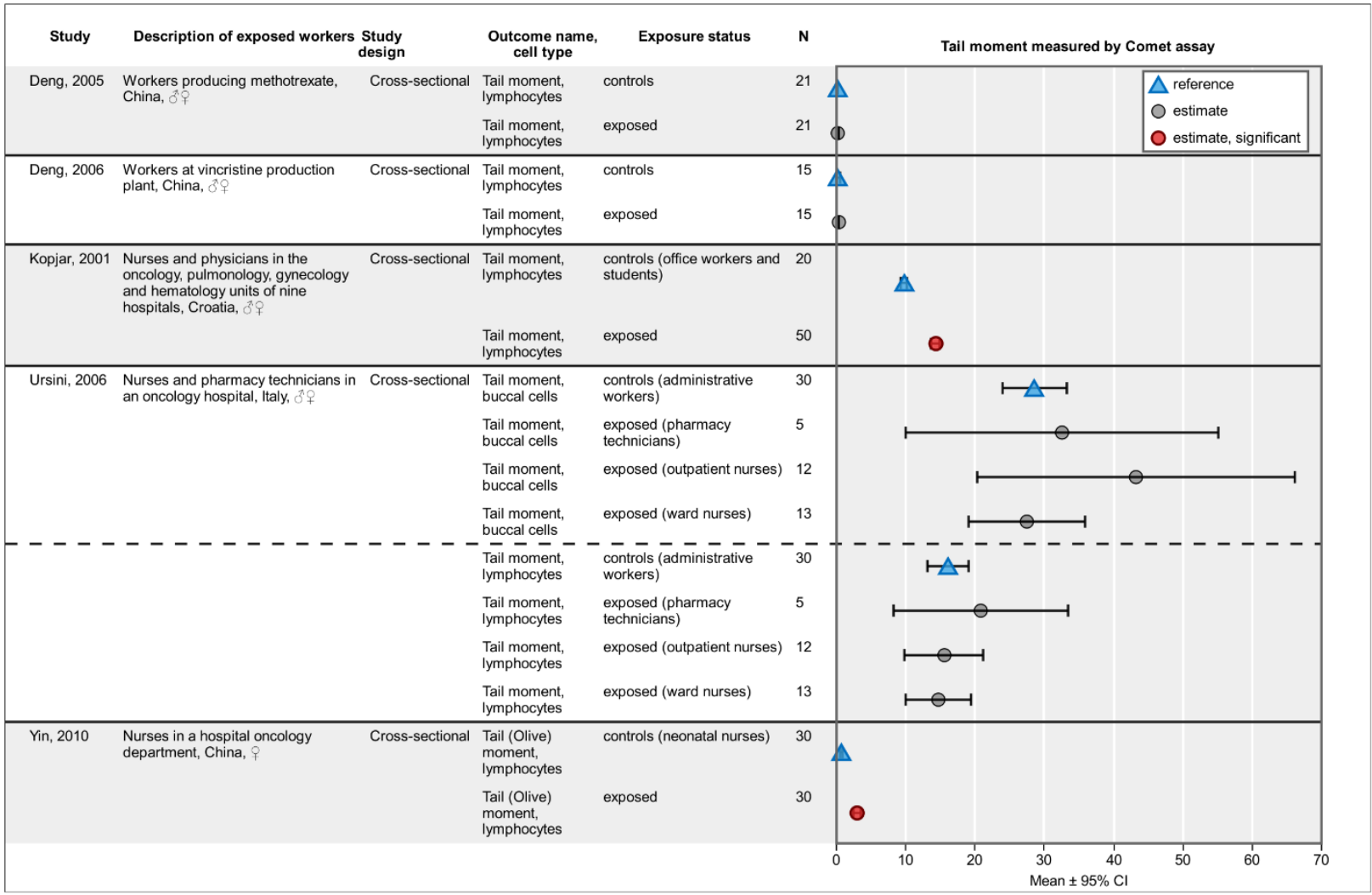

B

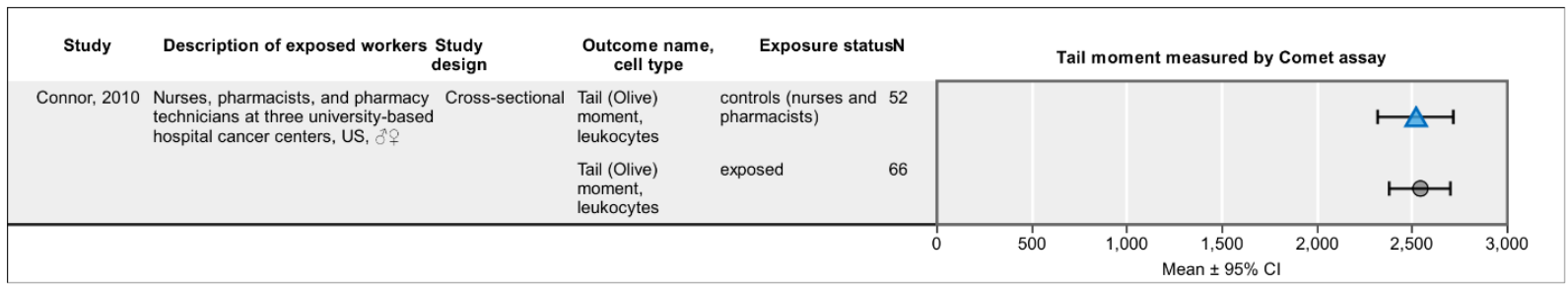

Figure D-17. Tail Moment Measured by the Comet Assay Associated with Occupational Exposure to Cancer Chemotherapy Agents for (A) Studies in the Expected Range and (B) Studies in Higher than the Expected Range

Interactive figure and additional study details in HAWC (NTP, 2015a). Controls refer to the unexposed, reference population. An additional study reported similar values of log-transformed mean tail moment in exposed nurses and controls following adjustment for age, smoking, coffee-drinking habits, hemoglobin, ionizing radiation, and hospital (data not graphed) (Sasaki et al. 2008). 


\section{A}

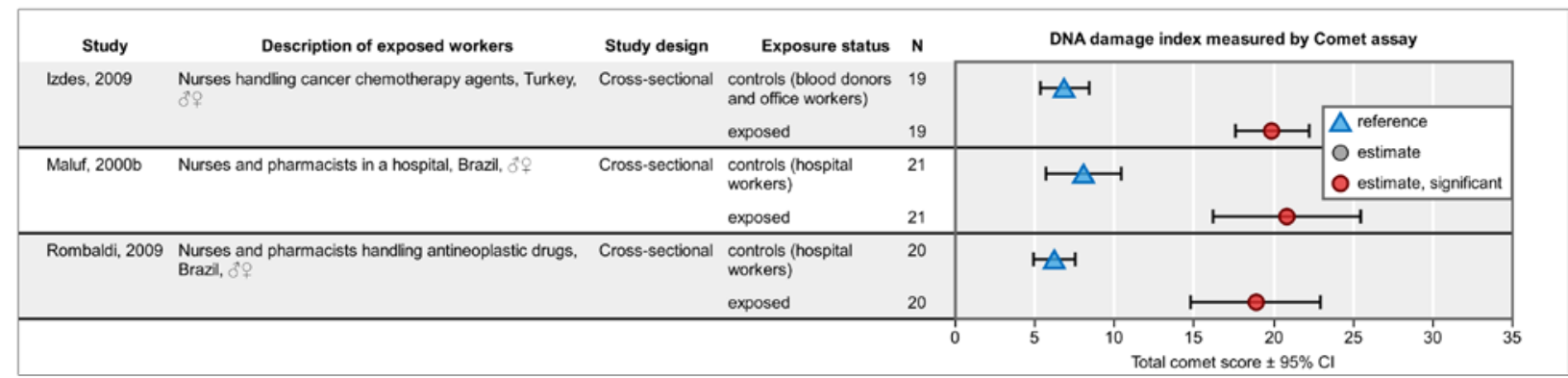

\section{B}

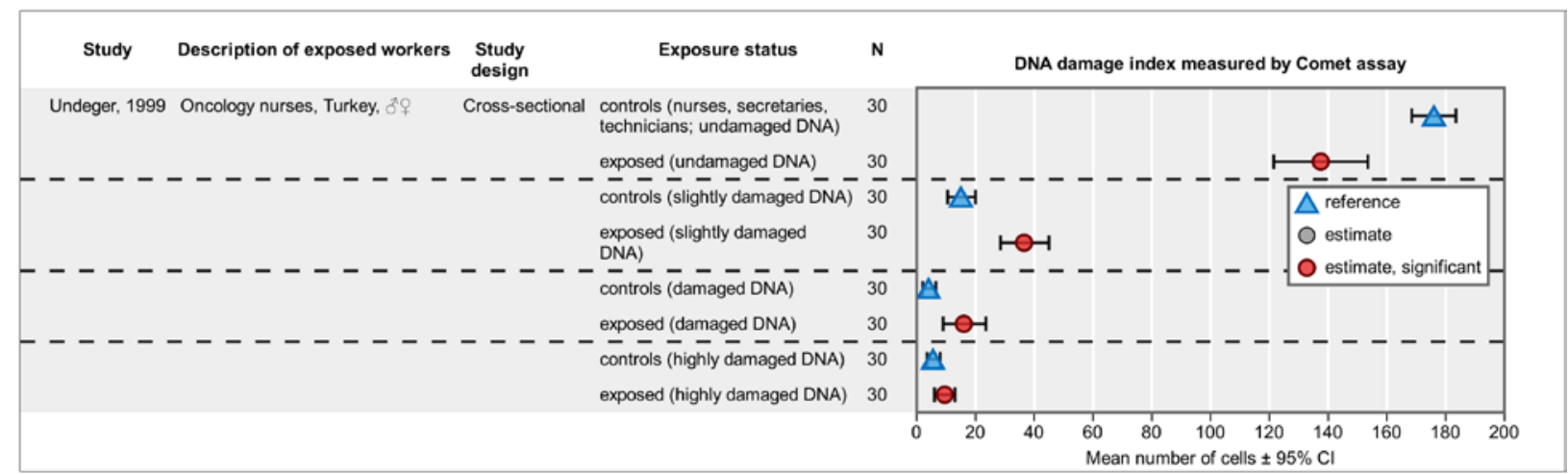

Figure D-18. DNA Damage Index Measured by the Comet Assay Associated with Occupational Exposure to Cancer Chemotherapy Agents Reported as (A) Mean Comet Score or (B) Mean Number of Cells

Interactive figure and additional study details in HAWC (NTP, 2015a). Controls refer to the unexposed, reference population. The mean comet scores (A) were calculated by binning the cells per category of DNA damage and multiplying the number of cells per category by a number per the level of DNA damage and summing up the numbers across the categories (see individual studies for more details). In addition, Mader et al. (2009) reported that a trend analysis for the entire observation period revealed a significantly greater $(\mathrm{p}=0.02)$ genotoxicity in the alkaline comet assay compared to unexposed subjects; no means or standard deviations were provided (data not graphed). 


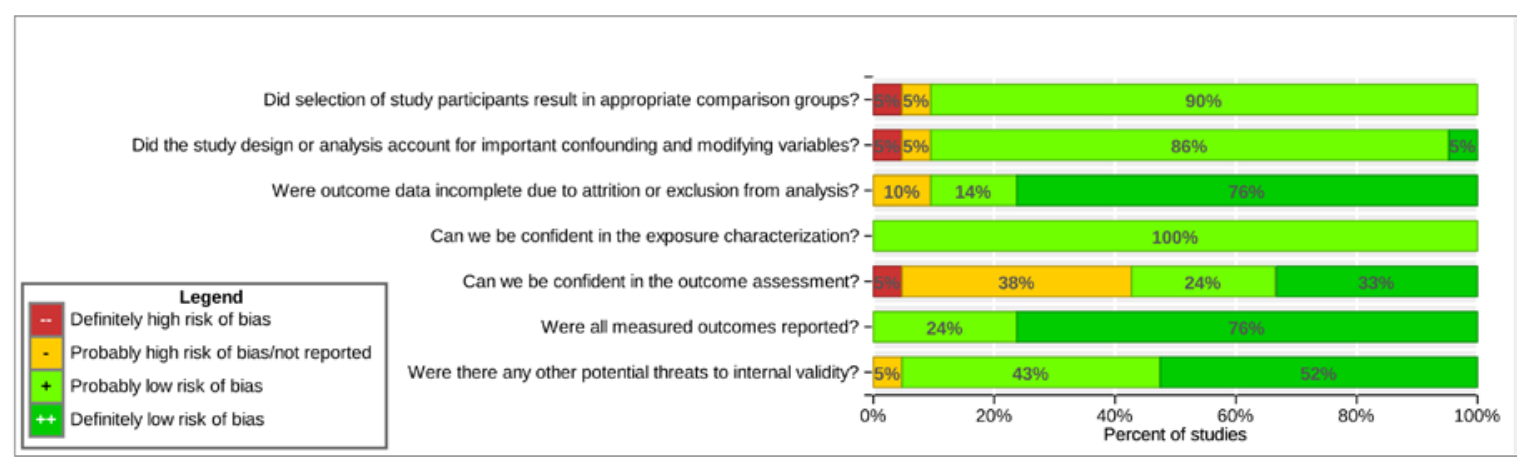

Figure D-19. Risk-of-bias Bar Graph for Included Studies Reporting DNA Damage Measured by the Comet Assay

Interactive figure and additional study details in HAWC (NTP, 2015a).

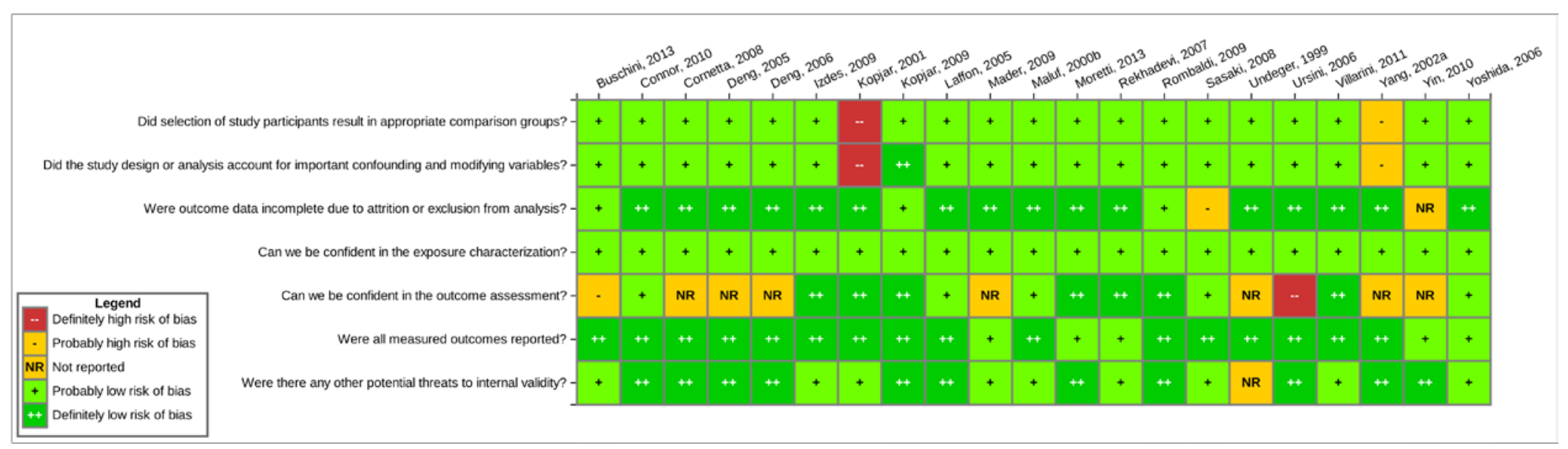

Figure D-20. Risk-of-bias Heat Map for Included Studies Reporting DNA Damage Measured by the Comet Assay

Interactive figure and additional study details in HAWC (NTP, 2015a). 


\section{Appendix E. Inadequate Evidence: Evidence Synthesis and Risk-of-bias Assessment}

\section{Table of Contents}

E.1. Acute Effects

E.2. Cancer

E.3. Immune Toxicity

E.4. Kidney, Liver, and Cardiovascular System Toxicity

E.5. Reproductive Toxicity, Other Than Spontaneous Abortion

\section{Tables}

Table E-1. Studies Reporting on Acute Effects .E-3

Table E-2. Studies Reporting on Incidence of Cancer. E-10

Table E-3. Studies Reporting on Immune System Toxicity $\mathrm{E}-14$

Table E-4. Studies Reporting on Kidney, Liver, or Cardiovascular System Toxicity. $\mathrm{E}-21$

Table E-5. Studies Reporting on Reproductive and Developmental Toxicity, Other Than Spontaneous Abortions

\section{Figures}

Figure E-1. Risk-of-bias Bar Graph for Studies Reporting on Acute Effects Associated with Occupational Exposure to Cancer Chemotherapy Agents

Figure E-2. Risk-of-bias Heat Map for Studies Reporting on Acute Effects Associated with Occupational Exposure to Cancer Chemotherapy Agents

Figure E-3. Risk-of-bias Bar Graph for Studies Reporting on Cancer Associated with Occupational Exposure to Cancer Chemotherapy Agents

Figure E-4. Risk-of-bias Heat Map for Studies Reporting on Cancer Associated with Occupational Exposure to Cancer Chemotherapy Agents

Figure E-5. Risk-of-bias Bar Graph for Studies Reporting on Immune System Effects Associated with Occupational Exposure to Cancer Chemotherapy Agents

Figure E-6. Risk-of-bias Heat Map for Studies Reporting on Immune System Effects Associated with Occupational Exposure to Cancer Chemotherapy Agents

Figure E-7. Risk-of-bias Bar Graph for Studies Reporting on Kidney, Liver, or Cardiovascular System Toxicity Associated with Occupational Exposure to Cancer Chemotherapy Agents

Figure E-8. Risk-of-bias Heat Map for Studies Reporting on Kidney, Liver, or Cardiovascular System Toxicity Associated with Occupational Exposure to Cancer Chemotherapy Agents

Figure E-9. Risk-of-bias Bar Graph for Studies Reporting on Reproductive and Developmental Toxicity, Other Than Spontaneous Abortion, Associated with Occupational Exposure to Cancer Chemotherapy....

Figure E-10. Risk-of-bias Heat Map for Studies Reporting on Reproductive and Developmental Toxicity, Other Than Spontaneous Abortion, Associated with Occupational Exposure to Cancer Chemotherapy. 


\section{E.1. Acute Effects}

The NTP evaluation identified nine cross-sectional studies reporting on acute health effects potentially associated with occupational exposure to cancer chemotherapy (Krstev et al. 2003; Medková 1991a; Rogers and Emmett 1987; Shahrasbi et al. 2014; Valanis et al. 1987; Valanis et al. 1993a; 1993b; Walusiak et al. 2003; Zhang et al. 2016). Acute effects are defined as health effects that develop rapidly, and these effects are often transient in nature and subside when the subject is no longer exposed. Given the wide range of symptoms that could be included under this category, there was a high degree of heterogeneity in type of effects reported, which limited the ability to compare across studies.

Summary: Based on the available studies, there is very low confidence in the body of evidence that occupational exposure to cancer chemotherapy agents is associated with the incidence of acute effects (Table E-1). There was a high degree of heterogeneity in the outcome assessment (i.e., symptoms evaluated), which resulted in few effects evaluated across all studies and the studies that evaluated related endpoints as a group reported mixed evidence of potential effects. For example, fewer than half of the studies that examined dermatological endpoints (e.g., skin rash, skin irritation or eczema) were significantly higher in exposed subjects versus controls (three of eight studies). Other examples of acute effects included significantly higher rates of hair loss reported in five of eight studies and significantly greater incidence of headache reported in four of six studies in exposed subjects relative to controls. The final rating of very low confidence in the body of evidence reflects downgrading for serious risk-of-bias concerns across multiple key risk-of-bias questions (e.g., majority of studies were rated probably high risk of bias for confounding, exposure characterization, and outcome assessment) (Figure E-1 and Figure E-2). 
Table E-1. Studies Reporting on Acute Effects

\begin{tabular}{|c|c|c|c|c|c|c|}
\hline Study & $\begin{array}{l}\text { Study Design } \\
\text { (Population, } \\
\text { Location, Year } \\
\text { Data Collected, } \\
\text { Sex) }\end{array}$ & $\begin{array}{c}\text { No. of } \\
\text { Participants }\end{array}$ & Exposure Measure & $\begin{array}{c}\text { Outcome } \\
\text { Assessment }\end{array}$ & Analysis & Results \\
\hline $\begin{array}{l}\text { Krstev et al. } \\
\text { (2003) }\end{array}$ & $\begin{array}{l}\text { Cross-sectional } \\
\text { (Nurses in } 8 \text { hospitals, } \\
\text { Serbia, year NR, F) }\end{array}$ & $\begin{array}{l}186 \text { exposed, } 77 \\
\text { unexposed }\end{array}$ & $\begin{array}{l}\text { Job title and self- } \\
\text { reported questionnaire; } \\
\text { head nurses reported on } \\
\text { work practice and work } \\
\text { conditions } \\
\text { Note: One hospital had } \\
\text { a vertical laminar } \\
\text { [flow] biological safety } \\
\text { cabinet, and some } \\
\text { concerns about its } \\
\text { maintenance; none of } \\
\text { the hospitals had a } \\
\text { separate room for drug } \\
\text { preparation; gloves } \\
\text { were used by } 81.7 \% \text { of } \\
\text { exposed nurses for drug } \\
\text { preparation and by } \\
60.1 \% \text { for drug } \\
\text { administration }\end{array}$ & $\begin{array}{l}\text { Self-reported } \\
\text { questionnaire }\end{array}$ & $\begin{array}{l}2 x 2 \text { table statistics } \\
\text { used to compute } \\
\text { relative risk (RR); } \\
\text { unconditional } \\
\text { logistic regression } \\
\text { analysis used to } \\
\text { adjust for } \\
\text { confounders: age } \\
\text { ( } \leq 35 \text { and } \geq 36 \text { years } \\
\text { old), smoking status } \\
\text { (smokers versus non- } \\
\text { smokers and ex- } \\
\text { smokers), and shift } \\
\text { work (daily shift vs. } \\
\text { rotating shift with } \\
\text { night work) }\end{array}$ & $\begin{array}{l}\text { Prevalence of several symptoms } \\
\text { assessed: (*significantly higher OR } \\
\text { compared to unexposed after } \\
\text { adjustment for age, smoking and } \\
\text { shiftwork) } \\
\text { Hair loss*, skin rash*, eczema*, skin } \\
\text { flush*, light headedness*, nausea*, } \\
\text { dizziness*, vomiting, eye irritation, } \\
\text { diarrhea, nasal congestion and runny } \\
\text { nose, headache, insomnia, menstrual } \\
\text { dysfunction, disappearance of } \\
\text { symptoms during the weekend*, mean } \\
\text { number of symptoms overall* and by } \\
\text { frequency of exposure } \\
\text { (daily } \geq \text { weekly > monthly)* }\end{array}$ \\
\hline $\begin{array}{l}\text { Medková } \\
\text { (1991a) }\end{array}$ & $\begin{array}{l}\text { Cross-sectional } \\
\text { (Physicians, nurses, } \\
\text { auxiliary workers, } \\
\text { Czech Republic, FM) }\end{array}$ & $\begin{array}{l}44 \text { exposed, } \\
32 \text { unexposed } \\
\text { partners of exposed } \\
\text { subjects }\end{array}$ & $\begin{array}{l}\text { Job title and } \\
\text { interviewer-led } \\
\text { questionnaire ( } \geq 5 \text { years, } \\
\mathrm{n}=25 ; 6-10 \text { years, } \\
\mathrm{n}=14 ; 11-15 \text { years, } \\
\mathrm{n}=4 ; \text { and } 16-20 \text { years, } \\
\mathrm{n}=1) \\
\text { Note: All exposed } \\
\text { personnel used gloves } \\
\text { and gowns; isolated } \\
\text { room for drug } \\
\text { preparation only used } \\
\text { by } 25 \% \text { of exposed } \\
\text { subjects }\end{array}$ & $\begin{array}{l}\text { Interviewer-led } \\
\text { questionnaire }\end{array}$ & NR & $\begin{array}{l}\text { No difference in prevalence of } \\
\text { dermatological afflictions (including } \\
\text { eczema) compared to unexposed } \\
\text { subjects }\end{array}$ \\
\hline
\end{tabular}


Systematic Review of Occupational Exposure to Cancer Chemotherapy Agents

\begin{tabular}{|c|c|c|c|c|c|c|}
\hline Study & $\begin{array}{c}\text { Study Design } \\
\text { (Population, } \\
\text { Location, Year } \\
\text { Data Collected, } \\
\text { Sex) }\end{array}$ & $\begin{array}{c}\text { No. of } \\
\text { Participants }\end{array}$ & Exposure Measure & $\begin{array}{c}\text { Outcome } \\
\text { Assessment }\end{array}$ & Analysis & Results \\
\hline $\begin{array}{l}\text { Rogers and } \\
\text { Emmett (1987) }\end{array}$ & $\begin{array}{l}\text { Cross-sectional } \\
\text { (Nurses in Oncology } \\
\text { Nursing Society, } \\
\text { United States, year } \\
\text { NR, F) }\end{array}$ & $\begin{array}{l}59 \text { exposed, } 64 \\
\text { unexposed } \\
\text { community health } \\
\text { nurses }\end{array}$ & $\begin{array}{l}\text { Job title and } \\
\text { interviewer-led } \\
\text { questionnaire } \\
\text { Note: Percent of nurses } \\
\text { using PPE all the time } \\
\text { was: } 5 \% \text { for masks, } 8 \% \\
\text { for gowns, } 20 \% \text { for } \\
\text { gloves; laminar flow } \\
\text { hoods were not used }\end{array}$ & $\begin{array}{l}\text { Interviewer-led } \\
\text { questionnaire }\end{array}$ & [Chi-squared test] & $\begin{array}{l}\text { Prevalence of } 21 \text { symptoms was } \\
\text { assessed, but authors did not report on } \\
\text { all the outcomes; significantly higher } \\
\text { prevalence ( } \mathrm{p} \leq 0.02 \text { ) of abdominal } \\
\text { pain, hair loss, and headaches on a } \\
\text { daily or weekly basis compared to } \\
\text { unexposed; skin rash tended } \\
\text { (p=0.06) to be more frequently } \\
\text { reported compared to unexposed } \\
\text { nurses }\end{array}$ \\
\hline $\begin{array}{l}\text { Shahrasbi et al. } \\
\text { (2014) }\end{array}$ & $\begin{array}{l}\text { Cross-sectional } \\
\text { (Nurses at } 9 \\
\text { professional and } \\
\text { specialized cancer } \\
\text { hospitals, Iran, year } \\
\text { NR, FM) }\end{array}$ & $\begin{array}{l}225 \text { exposed nurses, } \\
\text { unexposed nurses } \\
\text { from non-oncology } \\
\text { wards [number NR] }\end{array}$ & $\begin{array}{l}\text { Job title and self- } \\
\text { reported questionnaire } \\
\text { Note: Exposed nurses } \\
\text { were working for } \\
\geq 6 \text { months in oncology } \\
\text { ward; they used vertical } \\
\text { flow laminar flow } \\
\text { biological safety } \\
\text { cabinets; PPE was } \\
\text { available but not } \\
\text { regularly used }\end{array}$ & $\begin{array}{l}\text { Self-reported, } \\
\text { validated } \\
\text { questionnaire }\end{array}$ & NR & $\begin{array}{l}\text { Prevalence of several symptoms } \\
\text { assessed: (*significantly higher } \\
\text { compared to unexposed nurses) } \\
\text { hair loss*, irritation of mucous } \\
\text { membranes, eye irritation, skin } \\
\text { irritation*, dizziness, headache, } \\
\text { nausea and vomiting* }\end{array}$ \\
\hline
\end{tabular}




\begin{tabular}{|c|c|c|c|c|c|c|}
\hline Study & $\begin{array}{c}\text { Study Design } \\
\text { (Population, } \\
\text { Location, Year } \\
\text { Data Collected, } \\
\text { Sex) }\end{array}$ & $\begin{array}{c}\text { No. of } \\
\text { Participants }\end{array}$ & Exposure Measure & $\begin{array}{c}\text { Outcome } \\
\text { Assessment }\end{array}$ & Analysis & Results \\
\hline $\begin{array}{l}\text { Valanis et al. } \\
\text { (1987) }\end{array}$ & $\begin{array}{l}\text { Cross-sectional } \\
\text { (Hospital nurses, } \\
\text { United States, 1982- } \\
\text { 1984, sex NR) }\end{array}$ & $\begin{array}{l}134 \text { exposed: } 51 \\
\text { prepared and } \\
\text { administered the } \\
\text { drugs, } 39 \text { only } \\
\text { administered the } \\
\text { drugs, and } 44 \\
\text { handled patient } \\
\text { excreta; } 43 \\
\text { unexposed nurses }\end{array}$ & $\begin{array}{l}\text { Job title and self- } \\
\text { reported, validated } \\
\text { questionnaire (duration } \\
\text { NR) } \\
\text { Note: } 65 \% \text { of exposed } \\
\text { nurses used gloves } \\
\text { during drug } \\
\text { preparation and } 45 \% \\
\text { during administration } \\
\text { of drugs; skin contact } \\
\text { occurred on at least } \\
\text { half of drug-handling } \\
\text { events for 18\% of } \\
\text { exposed nurses during } \\
\text { drug preparation and } \\
25 \% \text { during } \\
\text { administration of } \\
\text { drugs; gowns, goggles } \\
\text { and masks were rarely } \\
\text { used }\end{array}$ & $\begin{array}{l}\text { Self-reported } \\
\text { questionnaire }\end{array}$ & $\begin{array}{l}\text { Chi-square test for } \\
\text { prevalence of } \\
\text { individual acute } \\
\text { symptoms; ANOVA } \\
\text { or t-test, as } \\
\text { appropriate, for } \\
\text { mean number of } \\
\text { symptoms or total } \\
\text { symptoms of acute } \\
\text { symptoms grouped } \\
\text { into one of } 5 \text { body } \\
\text { systems }\end{array}$ & $\begin{array}{l}\text { Prevalence of } 27 \text { acute effects } \\
\text { experienced in past } 3 \text { months: } \\
\text { (*significantly higher compared to } \\
\text { unexposed nurses) } \\
\text { Cardiac related: shortness of breath, } \\
\text { irregular heartbeat, swelling, elevated } \\
\text { blood pressure, chest pain; Infection } \\
\text { related*: chronic cough*, sore } \\
\text { throat*, throat/sinus irritation, } \\
\text { viral/other infection*; } \\
\text { Gastrointestinal*: nausea and } \\
\text { vomiting*, diarrhea, severe } \\
\text { constipation, colicky abdomen, } \\
\text { abdominal pain; General systemic*: } \\
\text { dizziness*, headache*, insomnia, pain } \\
\text { on urination, hair loss, nail } \\
\text { hyperpigmentation; Allergic*: allergic } \\
\text { reactions, dermatitis, eye irritation*; } \\
\text { Miscellaneous: fainting, anorexia, } \\
\text { back pain, other pain; significantly } \\
\text { higher mean total symptoms for high } \\
\text { exposure and lack of glove use } \\
\text { compared to unexposed nurses }\end{array}$ \\
\hline
\end{tabular}




\begin{tabular}{|c|c|c|c|c|c|c|}
\hline Study & $\begin{array}{l}\text { Study Design } \\
\text { (Population, } \\
\text { Location, Year } \\
\text { Data Collected, } \\
\text { Sex) }\end{array}$ & $\begin{array}{c}\text { No. of } \\
\text { Participants }\end{array}$ & Exposure Measure & $\begin{array}{c}\text { Outcome } \\
\text { Assessment }\end{array}$ & Analysis & Results \\
\hline $\begin{array}{l}\text { Valanis et al. } \\
\text { (1993a) }\end{array}$ & $\begin{array}{l}\text { Cross-sectional } \\
\text { (Nurses and nurse } \\
\text { aides in National } \\
\text { Surgical Adjuvant } \\
\text { Breast and Bowel } \\
\text { Project (NSABP), } \\
\text { USA, 1988-1990, F) }\end{array}$ & $\begin{array}{l}\text { 1,346 exposed (283 } \\
\text { skin contact, 1,063 } \\
\text { no skin contact), } 738 \\
\text { unexposed }\end{array}$ & $\begin{array}{l}\text { Job title and self- } \\
\text { reported, validated } \\
\text { questionnaire (duration } \\
\text { NR) } \\
\text { Note: 92\% of exposed } \\
\text { nurses used gloves: } \\
\text { during drug } \\
\text { preparation, 82\% } \\
\text { during administration } \\
\text { of drugs, } 89 \% \text { while } \\
\text { cleaning up spills, and } \\
\text { 75\% when handling } \\
\text { patient excreta; masks } \\
\text { and goggles primarily } \\
\text { during drug } \\
\text { preparation; gowns } \\
\text { worn only during drug } \\
\text { preparation and } \\
\text { cleaning up spills }\end{array}$ & $\begin{array}{l}\text { Self-reported } \\
\text { questionnaire, effects } \\
\text { observed during the } \\
\text { preceding } 3 \text { months }\end{array}$ & $\begin{array}{l}\text { Multiple linear } \\
\text { regression for total } \\
\text { number of symptoms } \\
\text { analyzed effects of } \\
\text { dose, duration, use of } \\
\text { protection, and skin } \\
\text { exposure and factors } \\
\text { of age, smoking } \\
\text { status, stress at work, } \\
\text { and body mass } \\
\text { index; likelihood } \\
\text { ratio tests compared } \\
\text { the goodness of fit of } \\
\text { competing models, } \\
\text { and large sample } \\
\text { maximum likelihood } \\
\text { theory computed } \\
\text { confidence limits and } \\
\text { p-values for specific } \\
\text { parameter estimates; } \\
\text { logistic regression } \\
\text { modelled the effects } \\
\text { of exposure and } \\
\text { control variables }\end{array}$ & $\begin{array}{l}\text { Prevalence of } 27 \text { acute effects } \\
\text { experienced in past } 3 \text { months: } \\
\text { (*significantly higher compared to } \\
\text { unexposed subjects) } \\
\text { Cardiac: shortness of breath, } \\
\text { irregular heartbeat, swelling of hands } \\
\text { and feet, chest pain, elevated blood } \\
\text { pressure; Infectious: chronic cough, } \\
\text { chronic throat irritation, cough when } \\
\text { lying down, fever; Gastrointestinal: } \\
\text { loss of appetite, nausea, vomiting, } \\
\text { diarrhea, colicky abdomen, } \\
\text { constipation; General systemic: hair } \\
\text { loss, nail hyperpigmentation, pain on } \\
\text { urination, decreased libido, malaise; } \\
\text { Allergic: allergic reactions, eye } \\
\text { irritation, rash; Neurological: } \\
\text { headache, dizziness, fainting, } \\
\text { insomnia; no difference in the } \\
\text { prevalence of acute effects compared } \\
\text { to unexposed subjects; however } \\
\text { exposed, nurses with skin exposure } \\
\text { tended (p = 0.07) to have more acute } \\
\text { effects in every category than exposed } \\
\text { nurses without skin exposure, except } \\
\text { neurological symptoms }\end{array}$ \\
\hline $\begin{array}{l}\text { Valanis et al. } \\
\text { (1993b) }\end{array}$ & $\begin{array}{l}\text { Cross-sectional } \\
\text { (Pharmacists and } \\
\text { pharmacy technicians } \\
\text { in NSABP, USA, } \\
\text { 1988-1990, FM) }\end{array}$ & $\begin{array}{l}533 \text { exposed, } 205 \\
\text { unexposed }\end{array}$ & $\begin{array}{l}\text { Job title and self- } \\
\text { reported, validated } \\
\text { questionnaire (duration } \\
\text { NR) } \\
\text { Note: Exposed } \\
\text { pharmacy personnel } \\
\text { used gloves at least } \\
75 \% \text { of the time when } \\
\text { handling drugs }\end{array}$ & $\begin{array}{l}\text { Self-reported } \\
\text { questionnaire, effects } \\
\text { observed during the } \\
\text { preceding } 3 \text { months }\end{array}$ & $\begin{array}{l}\text { Same analysis as } \\
\text { Valanis 1993a }\end{array}$ & $\begin{array}{l}\text { Prevalence of } 27 \text { acute effects } \\
\text { experienced in past } 3 \text { months same as } \\
\text { Valanis 1993a: significantly higher } \\
\text { prevalence of chronic throat irritation* } \\
\text { and diarrhea* compared to unexposed; } \\
\text { skin contact with cancer } \\
\text { chemotherapy agents was a significant } \\
\text { factor for mean total symptoms or } \\
\text { organ system subgroups }\end{array}$ \\
\hline
\end{tabular}




\begin{tabular}{|c|c|c|c|c|c|c|}
\hline Study & $\begin{array}{c}\text { Study Design } \\
\text { (Population, } \\
\text { Location, Year } \\
\text { Data Collected, } \\
\text { Sex) }\end{array}$ & $\begin{array}{c}\text { No. of } \\
\text { Participants }\end{array}$ & Exposure Measure & $\begin{array}{c}\text { Outcome } \\
\text { Assessment }\end{array}$ & Analysis & Results \\
\hline $\begin{array}{l}\text { Walusiak et al. } \\
\text { (2003) }\end{array}$ & $\begin{array}{l}\text { Cross-sectional } \\
\text { (Physicians, nurses, } \\
\text { orderlies, year NR, } \\
\text { Poland, FM) }\end{array}$ & $\begin{array}{l}103 \text { exposed, } 104 \\
\text { unexposed }\end{array}$ & $\begin{array}{l}\text { Job title and self- } \\
\text { reported questionnaire } \\
(11.0 \pm 9.3 \text { years })\end{array}$ & $\begin{array}{l}\text { Self-reported } \\
\text { questionnaire }\end{array}$ & $\begin{array}{l}\text { Mann-Whitney U- } \\
\text { test }\end{array}$ & $\begin{array}{l}\text { Prevalence the following acute } \\
\text { effects: } \\
\text { (*significantly higher compared to } \\
\text { unexposed subjects) } \\
\text { accelerated hair loss*, changes in the } \\
\text { fingernails (fragility, brittleness, } \\
\text { wrinkling), dermal changes } \\
\text { (bleed/bruising tendency), digestive } \\
\text { tract bleeding, and skin symptoms } \\
\text { (allergic or other etiology) }\end{array}$ \\
\hline $\begin{array}{l}\text { Zhang et al. } \\
\text { (2016) }\end{array}$ & $\begin{array}{l}\text { Cross-sectional } \\
\text { (Nurses, China, } 2011 \\
\text { pre-pharmacy } \\
\text { intravenous } \\
\text { admixture service } \\
\text { (pre-PIVAS) and } \\
2013 \text { (post-PIVAS) in } \\
\text { Hospital A, F) }\end{array}$ & $\begin{array}{l}\text { Pre-PIVAS: } 176 \\
\text { unexposed, } 46 \\
\text { exposed non- } \\
\text { oncology, } 123 \\
\text { exposed oncology; } \\
\text { post-PIVAS: } 182 \\
\text { unexposed, } 55 \\
\text { exposed non- } \\
\text { oncology, } 148 \\
\text { exposed oncology, } \\
\text { and } 190 \text { exposed } \\
\text { oncology nurses at } \\
\text { Hospital B) }\end{array}$ & $\begin{array}{l}\text { Job title and self- } \\
\text { reported questionnaire } \\
\text { ( } \geq 2 \text { years in current job) }\end{array}$ & $\begin{array}{l}\text { Self-reported } \\
\text { questionnaire }\end{array}$ & $\begin{array}{l}\text { [Student] t-test, Chi- } \\
\text { square, and } \\
\text { Wilcoxon [signed- } \\
\text { rank sum] test }\end{array}$ & $\begin{array}{l}\text { Significantly higher }(\mathrm{p}<0.01) \\
\text { prevalence of severe hair loss and oral } \\
\text { ulcers compared to unexposed nurses } \\
\text { in pre- and post-PIVAS studies; } \\
\text { however, the post-PIVAS prevalence } \\
\text { of these two acute effects were } \\
\text { significantly lower than pre-PIVAS } \\
\text { values ( }<\text { < } 0.05) \text {; however, the post- } \\
\text { PIVAS prevalence of oral ulcers was } \\
\text { significantly higher than Hospital B } \\
\text { without PIVAS }\end{array}$ \\
\hline
\end{tabular}

$\mathrm{F}$ = female, $\mathrm{M}$ = male, NR = not reported, NS = not significant, NSABP = National Surgical Adjuvant Breast and Bowel Project, OR = odds ratio, PIVAS = pharmacy intravenous admixture service. 


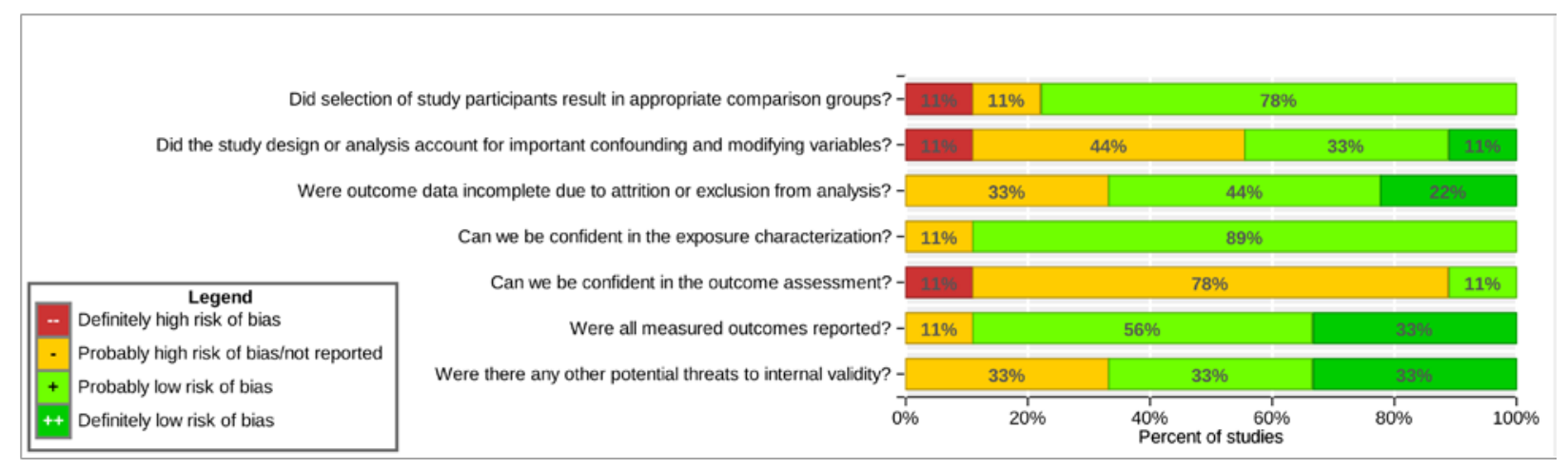

Figure E-1. Risk-of-bias Bar Graph for Studies Reporting on Acute Effects Associated with Occupational Exposure to Cancer Chemotherapy Agents

Interactive figure and additional study details in HAWC (NTP, 2015a).

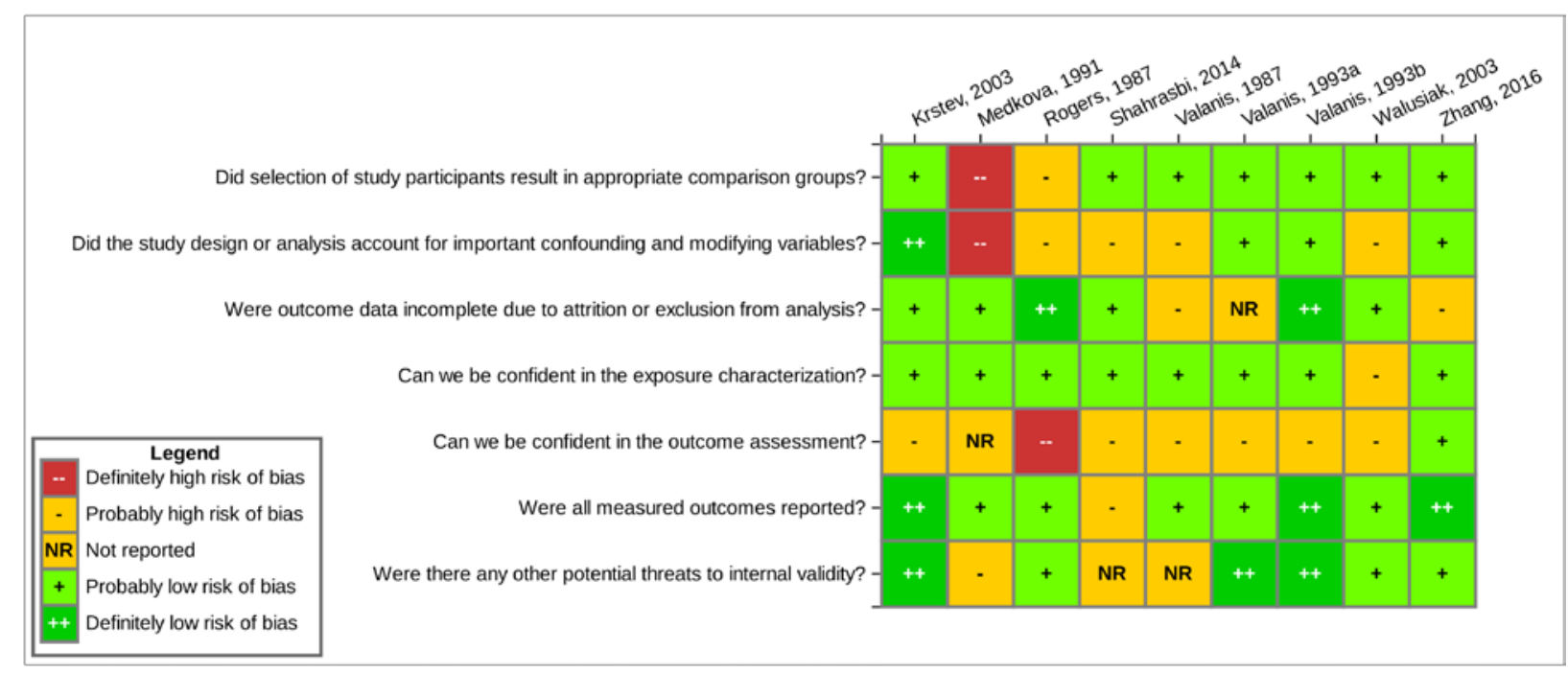

Figure E-2. Risk-of-bias Heat Map for Studies Reporting on Acute Effects Associated with Occupational Exposure to Cancer Chemotherapy Agents

Interactive figure and additional study details in HAWC (NTP, 2015a).

\section{E.2. Cancer}

NTP identified three studies reporting on cancer incidence (Gunnarsdóttir et al. 1997; Ratner et al. 2010; Skov et al. 1992). The confidence rating on the body of evidence were based on studies reporting on the cancers most commonly associated with exposure to cancer chemotherapy agents in patients administered the drugs for treatment of cancer: leukemia and/or lymphoma (Cogliano et al. 2011; Mistry et al. 2005; Pedersen-Bjergaard 2005; Pedersen-Bjergaard et al. 2002) as well as urinary bladder (chlornaphazine, cyclophosphamide), lung (MOPP: vincristineprednisone-nitrogen mustard-procarbazine combination therapy), and skin (azathioprine, nitrogen mustard) (Cogliano et al. 2011). In addition, an elevated risk of breast cancer has been observed in women working as nurses (Gunnarsdóttir and Rafnsson 1995; Lie et al. 2007). 
Summary: Based on the available studies, there is very low confidence in the body of evidence that occupational exposure to cancer chemotherapy agents is associated with cancer. There were very few studies of any one cancer site (Table E-2). For example, only one of two retrospective cohort studies of reporting on leukemia observed a significant association in nurses handling and administering cancer chemotherapy agents (Skov et al. 1992). Also, for some cancers, there were data reporting a lack of effect of exposure from study designs with lower initial confidence ratings (e.g., retrospective cohort studies), which limited the ability to reach level-of-evidence conclusions (e.g., Hodgkin lymphoma) (Ratner et al. 2010; Skov et al. 1992). The final rating of very low confidence in the body of evidence for other cancers reflects downgrading for serious risk-of-bias concerns across multiple key risk-of-bias questions (e.g., majority of studies were rated probably high risk of bias for confounding and exposure characterization) and only one to two available studies per each cancer type (Figure E-3 and Figure E-4). 
Table E-2. Studies Reporting on Incidence of Cancer

\begin{tabular}{|c|c|c|c|c|c|c|}
\hline Study & $\begin{array}{l}\text { Study Design } \\
\text { (Occupation, } \\
\text { Location, Year Data } \\
\text { Collected, Sex) }\end{array}$ & $\begin{array}{c}\text { No. of } \\
\text { Participants }\end{array}$ & $\begin{array}{l}\text { Exposure } \\
\text { Measure }\end{array}$ & $\begin{array}{l}\text { Outcome } \\
\text { Measured }\end{array}$ & Analysis & Results \\
\hline $\begin{array}{l}\text { Gunnarsdóttir et } \\
\text { al. (1997) }\end{array}$ & $\begin{array}{l}\text { Nested case-control in a } \\
\text { cohort of nurses } \\
\text { (Nurses, Iceland, 1955- } \\
1979, \text { F) }\end{array}$ & $\begin{array}{l}55 \text { cases and } 116 \\
\text { referents [number } \\
\text { exposed to cancer } \\
\text { chemotherapy } \\
\text { agents NR] }\end{array}$ & $\begin{array}{l}\text { National } \\
\text { occupational } \\
\text { records for } \\
\text { Icelandic nurses; } \\
\text { telephone- } \\
\text { interviews with } \\
\text { subjects or next of } \\
\text { kin for deceased } \\
\text { subjects }\end{array}$ & $\begin{array}{l}\text { National cancer } \\
\text { registry }\end{array}$ & $\begin{array}{l}\text { Odds ratio adjusted } \\
\text { for year of birth, } \\
\text { breast cancer in } \\
\text { first-degree relative, } \\
\text { marital status, and } \\
\text { nulliparity }\end{array}$ & $\begin{array}{l}\text { No significant association for breast } \\
\text { cancer (adjOR }=1.65,95 \% \\
\mathrm{CI}=0.53-5.17,7 \text { exposed cases) } \\
\text { compared to unexposed }\end{array}$ \\
\hline $\begin{array}{l}\text { Ratner et al. } \\
\text { (2010) }\end{array}$ & $\begin{array}{l}\text { Cohort, retrospective } \\
\text { (Female registered } \\
\text { nurses from British } \\
\text { Columbia, Canada, } \\
\text { 1974-2000, F) } \\
\text { Note: Subjects followed } \\
\text { until } 2000\end{array}$ & $\begin{array}{l}56,213 \text { nurses in } \\
\text { cohort: } \\
905 \text { exposed } \\
\text { (Method 1, ever } \\
\text { working in } \\
\text { oncology or a } \\
\text { cancer center) or } \\
7,635 \text { exposed } \\
\geq 15 \text { days (Method } \\
2 \text {, weighted } \\
\text { duration of } \\
\text { exposure) }\end{array}$ & $\begin{array}{l}\text { Two exposure } \\
\text { measures: } \\
\text { Method } 1 \text { : Years } \\
\text { employed in } \\
\text { oncology or at a } \\
\text { cancer center } \\
\text { (never, }<5 \text { years, } \\
\geq 5 \text { years, or ever) } \\
\text { and } \\
\text { Method 2: weighted } \\
\text { duration of } \\
\text { exposure based on } \\
\text { job responsibilities } \\
\text { ( }<15 \text { days, } \geq 15 \text { to } \\
60 \text { days, and } \\
>60 \text { days, or } \\
\geq 15 \text { days; } 15 \text { days } \\
\text { equivalent to } 1 \text { year } \\
\text { of work in a job } \\
\text { likely not exposed } \\
\text { and } 60 \text { days } \\
\text { equivalent to } 1 \text { year } \\
\text { of work in job } \\
\text { likely exposed to } \\
\text { cancer } \\
\text { chemotherapy } \\
\text { agents) }\end{array}$ & $\begin{array}{l}\text { Incidence of any } \\
\text { cancer (combined } \\
\text { and individual } \\
\text { types); cases } \\
\text { identified in the } \\
\text { Canadian cancer } \\
\text { registry }\end{array}$ & $\begin{array}{l}\text { Poisson regression; } \\
\text { adjusted for } \\
\text { calendar year and } \\
\text { age; lag of } 10 \text { years } \\
\text { applied for cancer } \\
\text { incidence; cancers } \\
\text { with } 3 \text { or fewer } \\
\text { cancers were not } \\
\text { statistically } \\
\text { analyzed }\end{array}$ & 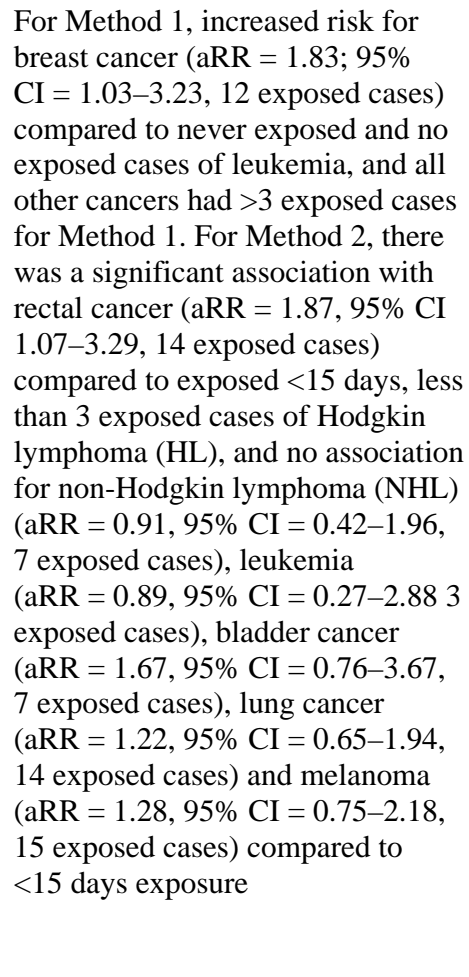 \\
\hline
\end{tabular}




\begin{tabular}{|c|c|c|c|c|c|c|}
\hline Study & $\begin{array}{c}\text { Study Design } \\
\text { (Occupation, } \\
\text { Location, Year Data } \\
\text { Collected, Sex) }\end{array}$ & $\begin{array}{c}\text { No. of } \\
\text { Participants }\end{array}$ & $\begin{array}{l}\text { Exposure } \\
\text { Measure }\end{array}$ & $\begin{array}{l}\text { Outcome } \\
\text { Measured }\end{array}$ & Analysis & Results \\
\hline Skov et al. (1992) & $\begin{array}{l}\text { Cohort, retrospective } \\
\text { (Nurses, Denmark, } \\
\text { 1973-1987, F) } \\
\text { Note: Follow-up until } \\
1987\end{array}$ & $\begin{array}{l}1,282 \text { exposed; } \\
\text { referents were } \\
\text { Danish national } \\
\text { cancer rates }\end{array}$ & $\begin{array}{l}\text { Employment } \\
\text { records of } 5 \\
\text { oncology hospitals, } \\
\text { and data from head } \\
\text { nurses regarding } \\
\text { drug handling and } \\
\text { administration of } \\
\text { individual subjects }\end{array}$ & $\begin{array}{l}\text { Medical records } \\
\text { (Danish Cancer } \\
\text { Registry); incidence } \\
\text { of any cancer } \\
\text { (combined), } \\
\text { leukemia, Hodgkin } \\
\text { lymphoma, non- } \\
\text { Hodgkin } \\
\text { lymphoma, multiple } \\
\text { myeloma, mycosis } \\
\text { fungoides }\end{array}$ & $\begin{array}{l}\text { Standard incidence } \\
\text { ratios }\end{array}$ & $\begin{array}{l}\text { Significant association for leukemia } \\
\text { (RR = 10.65, 95\% CI = } 1.29-38.5 \text {, } \\
2 \text { exposed cases), but not HL } \\
\text { (RR = 8.35, 95\% CI = 0.21-46.5, } 1 \\
\text { exposed case) compared to the } \\
\text { standard incidence ratio; one } \\
\text { exposed nurses had both leukemia } \\
\text { and JL was counted as } 2 \text { exposed } \\
\text { cases }\end{array}$ \\
\hline
\end{tabular}

aRR = adjusted relative risk, $\mathrm{CI}=$ confidence interval, $\mathrm{F}=$ female, $\mathrm{HL}=$ Hodgkin lymphoma, $\mathrm{M}=$ male, $\mathrm{NHL}=$ non-Hodgkin lymphoma, No. = number, RR = relative risk

${ }^{a}$ In Method 2 of Ratner et al. (2010), 15 days is equivalent to 1 year of work in a position not likely to be exposed and 60 days is equivalent to full time work in a position likely to be exposed. 


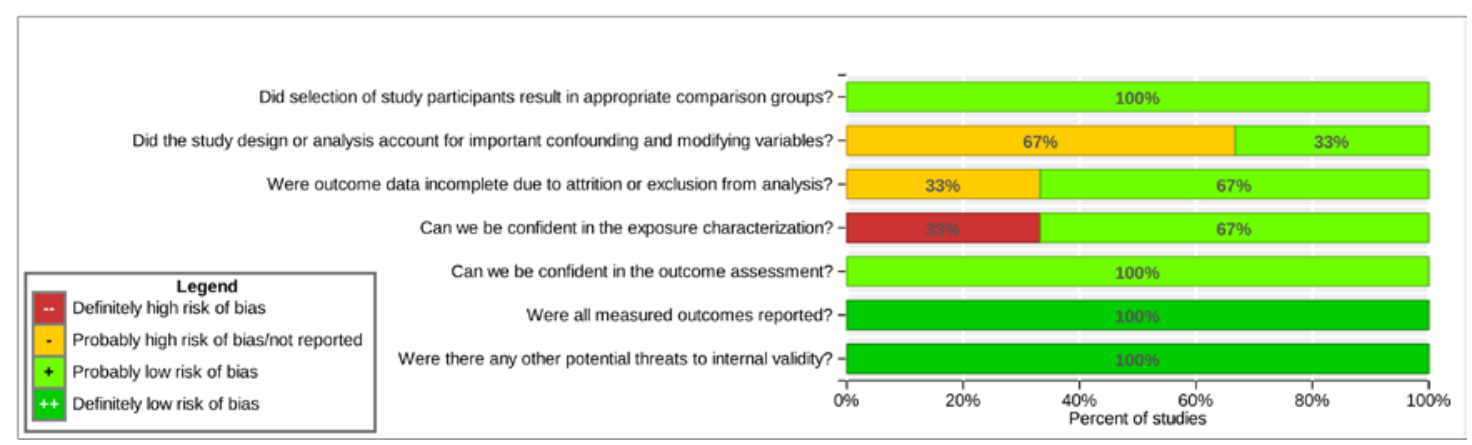

Figure E-3. Risk-of-bias Bar Graph for Studies Reporting on Cancer Associated with Occupational Exposure to Cancer Chemotherapy Agents

Interactive figure and additional study details in HAWC (NTP, 2015a).

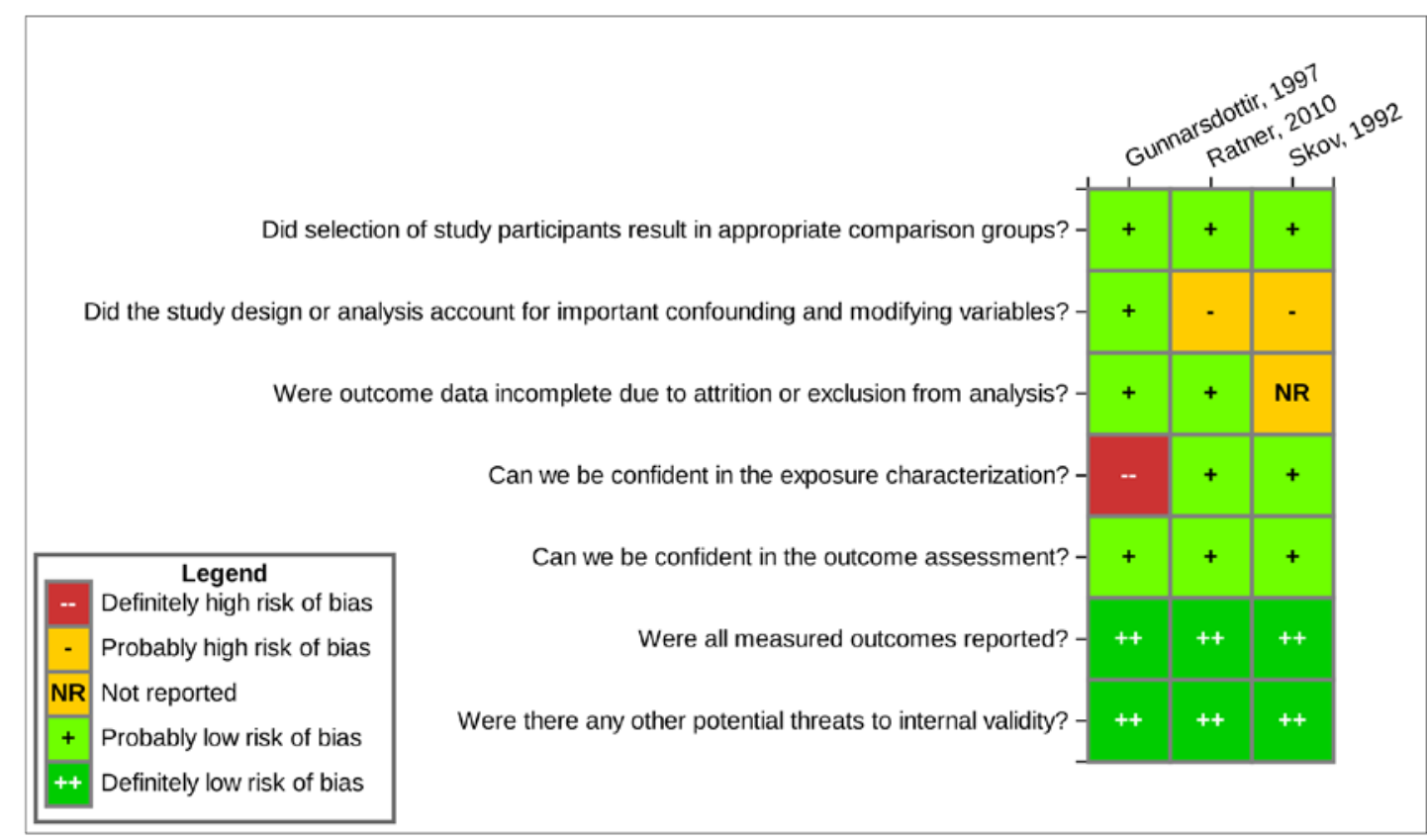

Figure E-4. Risk-of-bias Heat Map for Studies Reporting on Cancer Associated with Occupational Exposure to Cancer Chemotherapy Agents

Interactive figure and additional study details in HAWC (NTP, 2015a).

\section{E.3. Immune Toxicity}

The NTP evaluation identified 11 cross-sectional studies reporting effects on the immune system (Biró et al. 2011; Bolognesi et al. 2005; Caciari et al. 2012; Jeebhay et al. 1993; Jochimsen et al. 1988; Li et al. 2005; Medková 1991a; Spatari et al. 2005; Valanis et al. 1987; Walusiak et al. 2003; Zhang et al. 2016). The effects included any immune-related diseases (e.g., asthma, autoimmune disease). Alterations in immune-related parameters were also reviewed, including but not limited to: white blood cell differential count, oxidative burst potential, cytokine levels, and natural killer cell function. 
Summary: Based on the available studies, there is very low confidence in the body of evidence that occupational exposure to cancer chemotherapy agents is associated with higher incidence of immune-related diseases or alterations of immune-related parameters. The ability to compare among studies was limited due to heterogeneity in the outcomes assessed. For example, only one study reported on an immune-related disease (asthma) and most of the studies investigating possible alterations of immune-related parameters did not measure similar endpoints (Table E-3). Of the few studies that measured a similar endpoint, there were inconsistent results (e.g., only one of five studies measuring white blood cell counts reported significantly lower counts in exposed subjects versus controls). The final rating of very low confidence in the body of evidence confidence reflects downgrading for serious risk-of-bias concerns across multiple key risk-of-bias questions (e.g., majority of studies were rated probably high risk of bias for confounding), heterogeneity in the outcomes measured, and inconsistencies in the outcomes reported (Figure E-5 and Figure E-6). 
Table E-3. Studies Reporting on Immune System Toxicity

\begin{tabular}{|c|c|c|c|c|c|c|}
\hline Study & $\begin{array}{l}\text { Study Design } \\
\text { (Population, } \\
\text { Location, Year } \\
\text { Data Collected, } \\
\text { Sex) }\end{array}$ & $\begin{array}{c}\text { No. of } \\
\text { Participants }\end{array}$ & $\begin{array}{c}\text { Exposure } \\
\text { Characterization } \\
\text { (Duration) }\end{array}$ & $\begin{array}{c}\text { Outcome } \\
\text { Assessment }\end{array}$ & Analysis & $\begin{array}{c}\text { Results } \\
\text { (Outcomes Measured in Bold) }\end{array}$ \\
\hline Biró et al. (2011) & $\begin{array}{l}\text { Cross-sectional } \\
\text { (Nurses, Hungary, } \\
\text { year NR, sex NR) }\end{array}$ & $\begin{array}{l}308 \text { exposed, } 98 \\
\text { unexposed }\end{array}$ & $\begin{array}{l}\text { Job title and } \\
\text { interviewer-led } \\
\text { questionnaire } \\
\text { ( } 9.0 \pm 0.5 \text { years })\end{array}$ & $\begin{array}{l}\text { Medical tests } \\
\text { conducted using } \\
\text { flow cytometry }\end{array}$ & Student's t-test & 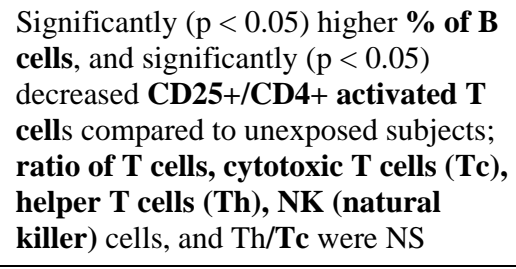 \\
\hline $\begin{array}{l}\text { Bolognesi et al. } \\
\text { (2005) }\end{array}$ & $\begin{array}{l}\text { Cross-sectional } \\
\text { (Nurses, IMPEA } \\
\text { project, Italy, year } \\
\text { NR, FM) }\end{array}$ & $\begin{array}{l}50 \text { exposed, } 48 \\
\text { unexposed }\end{array}$ & $\begin{array}{l}\text { Job title, [self- } \\
\text { reported] } \\
\text { questionnaire, and } \\
\text { biomonitoring } \\
\text { (10.2 years) } \\
\text { Note: Nurses } \\
\text { assigned to } \\
\text { preparation and } \\
\text { administration of } \\
\text { agents; adequate } \\
\text { safety equipment and } \\
\text { PPE were used }\end{array}$ & $\begin{array}{l}\text { Medical tests } \\
\text { conducted using } \\
\text { flow cytometry }\end{array}$ & $\begin{array}{l}\text { Mann-Whitney U- } \\
\text { test }\end{array}$ & $\begin{array}{l}\text { No difference in \% NK cells, } \\
\text { peripheral lymphomonocytes, } \\
\text { regulatory T cell (CD25), and IL-6 } \\
\text { compared to unexposed subjects }\end{array}$ \\
\hline Caciari et al. (2012) & $\begin{array}{l}\text { Cross-sectional } \\
\text { (Healthcare workers } \\
\text { (doctors, nurses, } \\
\text { social and health } \\
\text { assistants), Italy, } \\
\text { year NR, FM) }\end{array}$ & $\begin{array}{l}57 \text { exposed, } 57 \\
\text { unexposed }\end{array}$ & $\begin{array}{l}\text { Job title only } \\
\text { (duration NR) }\end{array}$ & Medical tests & $\begin{array}{l}\text { Student t-test, Chi- } \\
\text { square analysis }\end{array}$ & $\begin{array}{l}\text { Significantly lower levels of monocytes } \\
\text { (possible myelosuppression) compared } \\
\text { to unexposed subjects; red blood cell } \\
\text { populations or other white blood cell } \\
\text { type counts NS }\end{array}$ \\
\hline
\end{tabular}


Systematic Review of Occupational Exposure to Cancer Chemotherapy Agents

\begin{tabular}{|c|c|c|c|c|c|c|}
\hline Study & $\begin{array}{l}\text { Study Design } \\
\text { (Population, } \\
\text { Location, Year } \\
\text { Data Collected, } \\
\text { Sex) }\end{array}$ & $\begin{array}{c}\text { No. of } \\
\text { Participants }\end{array}$ & $\begin{array}{c}\text { Exposure } \\
\text { Characterization } \\
\text { (Duration) }\end{array}$ & $\begin{array}{c}\text { Outcome } \\
\text { Assessment }\end{array}$ & Analysis & $\begin{array}{c}\text { Results } \\
\text { (Outcomes Measured in Bold) }\end{array}$ \\
\hline $\begin{array}{l}\text { Jeebhay et al. } \\
\text { (1993) }\end{array}$ & $\begin{array}{l}\text { Cross-sectional } \\
\text { (Pharmaceutical } \\
\text { plant workers, } \\
\text { South Africa, year } \\
\text { NR, M) }\end{array}$ & $\begin{array}{l}6 \text { exposed to } \\
\text { azathioprine, } 17 \\
\text { unexposed }\end{array}$ & $\begin{array}{l}\text { Job title and [self- } \\
\text { reported] } \\
\text { questionnaire, } \\
\text { biomonitoring, and } \\
\text { environmental } \\
\text { monitoring } \\
\text { Note: Exposed } \\
\text { workers in 2-week } \\
\text { production campaign: } \\
\text { measured at baseline, } \\
\text { day 3, end, and } \\
3 \text { months after end }\end{array}$ & $\begin{array}{l}\text { Medical tests } \\
\text { conducted by a } \\
\text { commercial } \\
\text { laboratory }\end{array}$ & ANOVA & $\begin{array}{l}\text { Significantly fewer reticulocytes at the } \\
\text { end of the production run, and } \\
\text { significantly fewer neutrophils at } \\
3 \text { months following the production run } \\
\text { compared to unexposed subjects; } \\
\text { number of platelets or white blood } \\
\text { cell count NS }\end{array}$ \\
\hline $\begin{array}{l}\text { Jochimsen et al. } \\
\text { (1988) }\end{array}$ & $\begin{array}{l}\text { Cross-sectional } \\
\text { (Nurses, USA, year } \\
\text { NR, sex NR) }\end{array}$ & $\begin{array}{l}18 \text { exposed, } 18 \\
\text { unexposed }\end{array}$ & $\begin{array}{l}\text { Job title, work task, } \\
\text { drug handling, and } \\
\text { PPE usage [method } \\
\text { NR] (4.5 years, range } \\
1-10 \text { years) } \\
\text { Note: PPE not } \\
\text { regularly used and no } \\
\text { hoods or vapor } \\
\text { barriers were used }\end{array}$ & $\begin{array}{l}\text { Medical tests } \\
\text { conducted using } \\
\text { flow cytometry }\end{array}$ & Student's t-test & $\begin{array}{l}\text { Trend, though NS, of lower neutrophil, } \\
\text { monocytes, platelet counts, and } \\
\text { neutrophil reserve compared to } \\
\text { unexposed nurses }\end{array}$ \\
\hline
\end{tabular}




\begin{tabular}{|c|c|c|c|c|c|c|}
\hline Study & $\begin{array}{l}\text { Study Design } \\
\text { (Population, } \\
\text { Location, Year } \\
\text { Data Collected, } \\
\text { Sex) }\end{array}$ & $\begin{array}{c}\text { No. of } \\
\text { Participants }\end{array}$ & $\begin{array}{l}\text { Exposure } \\
\text { Characterization } \\
\text { (Duration) }\end{array}$ & $\begin{array}{c}\text { Outcome } \\
\text { Assessment }\end{array}$ & Analysis & $\begin{array}{c}\text { Results } \\
\text { (Outcomes Measured in Bold) }\end{array}$ \\
\hline Li et al. (2005) & $\begin{array}{l}\text { Cross-sectional } \\
\text { (Nurses, China, } \\
\text { years NR, F) }\end{array}$ & $\begin{array}{l}30 \text { exposed, } 30 \\
\text { unexposed }\end{array}$ & $\begin{array}{l}\text { Job title, drug } \\
\text { handling, and PPE } \\
\text { usage [method NR] } \\
\text { (14 years) } \\
\text { Note: Exposed nurses } \\
\text { handled these agents } \\
\geq 5 \text { years at minimum } \\
5 \text { times per day } \\
\text { without taking } \\
\text { protective measures }\end{array}$ & $\begin{array}{l}\text { Medical tests } \\
\text { conducted using } \\
\text { flow cytometry }\end{array}$ & t-test & $\begin{array}{l}\text { Significantly fewer helper } \mathbf{T} \text { cells } \\
\text { (CD4+) in exposed versus control } \\
\text { nurses; other T lymphocyte cell types } \\
\text { (CD8+, CD4+/CD8+, CD3) or NK } \\
\text { cells NS }\end{array}$ \\
\hline Medková (1991a) & $\begin{array}{l}\text { Cross-sectional } \\
\text { (Nurses, physicians, } \\
\text { cleaning staff, } \\
\text { Czech Republic, } \\
\text { years NR, FM) }\end{array}$ & $\begin{array}{l}44 \text { exposed, } 32 \\
\text { unexposed partners } \\
\text { of exposed subjects }\end{array}$ & $\begin{array}{l}\text { Job title and } \\
\text { interviewer-led } \\
\text { questionnaire } \\
\text { ( } \geq 5 \text { years, } \mathrm{n}=25 ; 6- \\
10 \text { years, } \mathrm{n}=14 ; 11- \\
15 \text { years, } \mathrm{n}=4 \text {; and } \\
16-20 \text { years, } \mathrm{n}=1 \text { ) } \\
\text { Note: All exposed } \\
\text { personnel used gloves } \\
\text { and gowns; isolated } \\
\text { room for drug } \\
\text { preparation only used } \\
\text { by } 25 \% \text { of exposed } \\
\text { subjects }\end{array}$ & $\begin{array}{l}\text { Interviewer-led } \\
\text { questionnaire }\end{array}$ & NR & $\begin{array}{l}\text { Higher incidence of allergy including } \\
\text { bronchial asthma in exposed nurses } \\
\text { versus controls, but statistical } \\
\text { significance unknown }\end{array}$ \\
\hline Spatari et al. (2005) & $\begin{array}{l}\text { Cross-sectional } \\
\text { (Healthcare } \\
\text { workers, Italy, } \\
\text { years NR, FM) }\end{array}$ & $\begin{array}{l}17 \text { exposed, } 17 \\
\text { unexposed }\end{array}$ & $\begin{array}{l}\text { Job title only } \\
(13.25 \pm 8.16 \text { years })\end{array}$ & Elisa kit & $\begin{array}{l}\text { One-way ANOVA, } \\
\text { Student-Newman } \\
\text { Keuls test }\end{array}$ & $\begin{array}{l}\text { Significantly higher interleukin-15 } \\
\text { levels in exposed relative to control } \\
\text { subjects }\end{array}$ \\
\hline
\end{tabular}


Systematic Review of Occupational Exposure to Cancer Chemotherapy Agents

\begin{tabular}{|c|c|c|c|c|c|c|}
\hline Study & $\begin{array}{l}\text { Study Design } \\
\text { (Population, } \\
\text { Location, Year } \\
\text { Data Collected, } \\
\text { Sex) }\end{array}$ & $\begin{array}{c}\text { No. of } \\
\text { Participants }\end{array}$ & $\begin{array}{c}\text { Exposure } \\
\text { Characterization } \\
\text { (Duration) }\end{array}$ & $\begin{array}{c}\text { Outcome } \\
\text { Assessment }\end{array}$ & Analysis & $\begin{array}{c}\text { Results } \\
\text { (Outcomes Measured in Bold) }\end{array}$ \\
\hline $\begin{array}{l}\text { Valanis et al. } \\
\text { (1987) }\end{array}$ & $\begin{array}{l}\text { Cross-sectional } \\
\text { (Pharmacists and } \\
\text { pharmacy } \\
\text { technicians in } \\
\text { NSABP, USA, } \\
\text { 1988-1990, FM) }\end{array}$ & $\begin{array}{l}21 \text { exposed, } 13 \\
\text { unexposed }\end{array}$ & $\begin{array}{l}\text { Job title and self- } \\
\text { reported, validated } \\
\text { questionnaire } \\
\text { (duration NR) } \\
\text { Note: } 65 \% \text { of exposed } \\
\text { nurses used gloves } \\
\text { during drug } \\
\text { preparation and } 45 \% \\
\text { during administration } \\
\text { of drugs; skin contact } \\
\text { occurred on at least } \\
\text { half of drug-handling } \\
\text { events for 18\% of } \\
\text { exposed nurses and } \\
\text { 25\% of nurses during } \\
\text { administration of } \\
\text { drugs; gowns, } \\
\text { goggles and masks } \\
\text { were rarely used }\end{array}$ & $\begin{array}{l}\text { Medical tests } \\
\text { conducted in single } \\
\text { laboratory by } \\
\text { technician blind to } \\
\text { exposure group }\end{array}$ & Chi-squared test & $\begin{array}{l}\text { Similar total white cell counts between } \\
\text { control and exposed subjects; however, } \\
\text { an analysis of subgroups of white cell } \\
\text { counts }(<5,600,5,600-7,000,>7,000) \\
\text { revealed that significantly more } \\
\text { exposed nurses had white blood cell } \\
\text { counts of }<1,500 \text { cells than control } \\
\text { nurses }\end{array}$ \\
\hline $\begin{array}{l}\text { Walusiak et al. } \\
\text { (2003) }\end{array}$ & $\begin{array}{l}\text { Cross-sectional } \\
\text { (Nurses, physicians, } \\
\text { orderlies, year NR, } \\
\text { Poland, FM) }\end{array}$ & $\begin{array}{l}104 \text { exposed, } 103 \\
\text { unexposed }\end{array}$ & $\begin{array}{l}\text { Job title and self- } \\
\text { reported } \\
\text { questionnaire } \\
(11.0 \pm 9.3 \text { years, } \\
\text { range } 5-44 \text { years) }\end{array}$ & Medical tests & $\begin{array}{l}\text { Mann-Whitney U- } \\
\text { test }\end{array}$ & $\begin{array}{l}\text { Similar values between exposed and } \\
\text { control subjects for all immune and } \\
\text { hematopoietic parameters measured } \\
\text { including: white blood cell count, red } \\
\text { blood cells count and platelet count }\end{array}$ \\
\hline
\end{tabular}


Systematic Review of Occupational Exposure to Cancer Chemotherapy Agents

\begin{tabular}{|c|c|c|c|c|c|c|}
\hline Study & $\begin{array}{c}\text { Study Design } \\
\text { (Population, } \\
\text { Location, Year } \\
\text { Data Collected, } \\
\text { Sex) }\end{array}$ & $\begin{array}{l}\text { No. of } \\
\text { Participants }\end{array}$ & $\begin{array}{c}\text { Exposure } \\
\text { Characterization } \\
\text { (Duration) }\end{array}$ & $\begin{array}{l}\text { Outcome } \\
\text { Assessment }\end{array}$ & Analysis & $\begin{array}{c}\text { Results } \\
\text { (Outcomes Measured in Bold) }\end{array}$ \\
\hline Zhang et al. (2016) & $\begin{array}{l}\text { Cross-sectional } \\
\text { (Nurses, China, } \\
2011 \text { pre-PIVAS } \\
\text { and } 2013 \text { (post- } \\
\text { PIVAS) in Hospital } \\
\text { A, F) }\end{array}$ & $\begin{array}{l}\text { Pre-PIVAS: } 88 \\
\text { unexposed, } 33 \\
\text { exposed non- } \\
\text { oncology, } 101 \\
\text { exposed oncology; } \\
\text { post-PIVAS: } 55 \\
\text { unexposed, } 25 \\
\text { exposed non- } \\
\text { oncology, } 85 \\
\text { exposed oncology, } \\
\text { and } 144 \text { exposed } \\
\text { oncology nurses at } \\
\text { Hospital B) }\end{array}$ & $\begin{array}{l}\text { Job title and self- } \\
\text { reported } \\
\text { questionnaire } \\
\text { ( } \geq 2 \text { years in current } \\
\text { job) } \\
\text { Note: All exposed } \\
\text { nurses involved in } \\
\text { mixing and/or } \\
\text { administration of } \\
\text { cancer chemotherapy } \\
\text { agents }\end{array}$ & Medical tests & $\begin{array}{l}\text { t-test, Chi-square, } \\
\text { and Wilcoxon }\end{array}$ & $\begin{array}{l}\text { Significantly lower mean white blood } \\
\text { cell counts in oncology nurses } \\
\text { compared to controls prior to the } \\
\text { implementation of a PIVAS; after } \\
\text { implementation of PIVAS, the white } \\
\text { blood cell counts were similar between } \\
\text { the two groups in the same hospital. } \\
\text { The number of white blood cell } \\
\text { counts, platelets, and red blood cells } \\
\text { counts were higher in controls and } \\
\text { oncology numbers after PIVAS } \\
\text { implementation than before. Finally, } \\
\text { oncology nurses in a second hospital } \\
\text { (Hospital B) without PIVAS had } \\
\text { significantly lower numbers of white } \\
\text { bloods cells, platelets, and red blood } \\
\text { cells compared to the oncology nurses } \\
\text { post-PIVAS at Hospital A }\end{array}$ \\
\hline
\end{tabular}




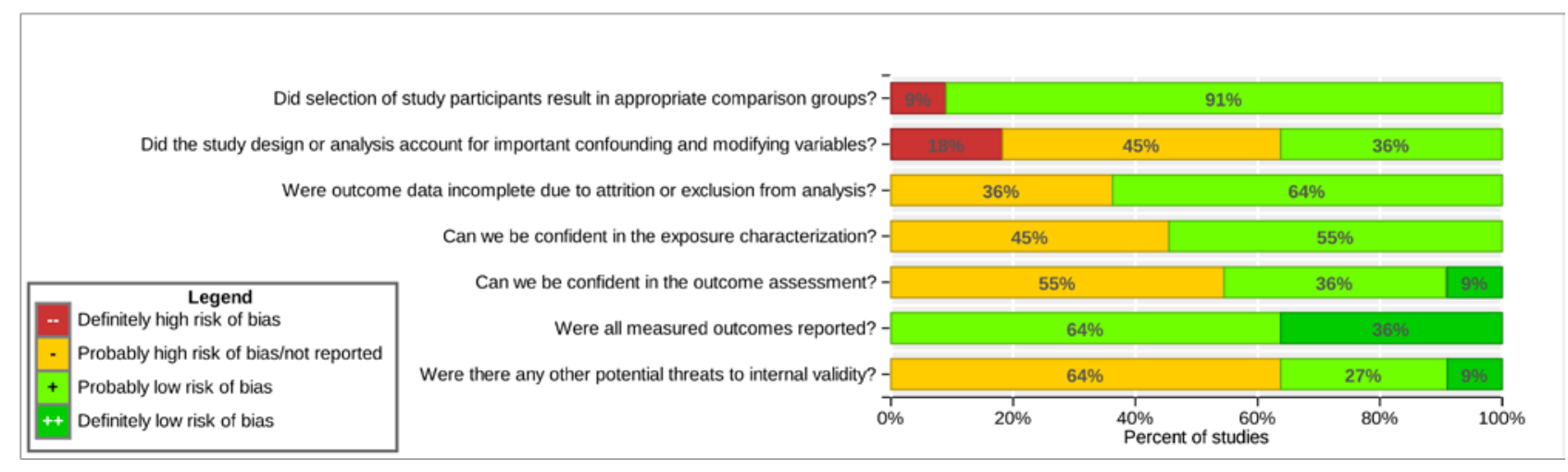

Figure E-5. Risk-of-bias Bar Graph for Studies Reporting on Immune System Effects Associated with Occupational Exposure to Cancer Chemotherapy Agents

Interactive figure and additional study details in HAWC (NTP, 2015a).

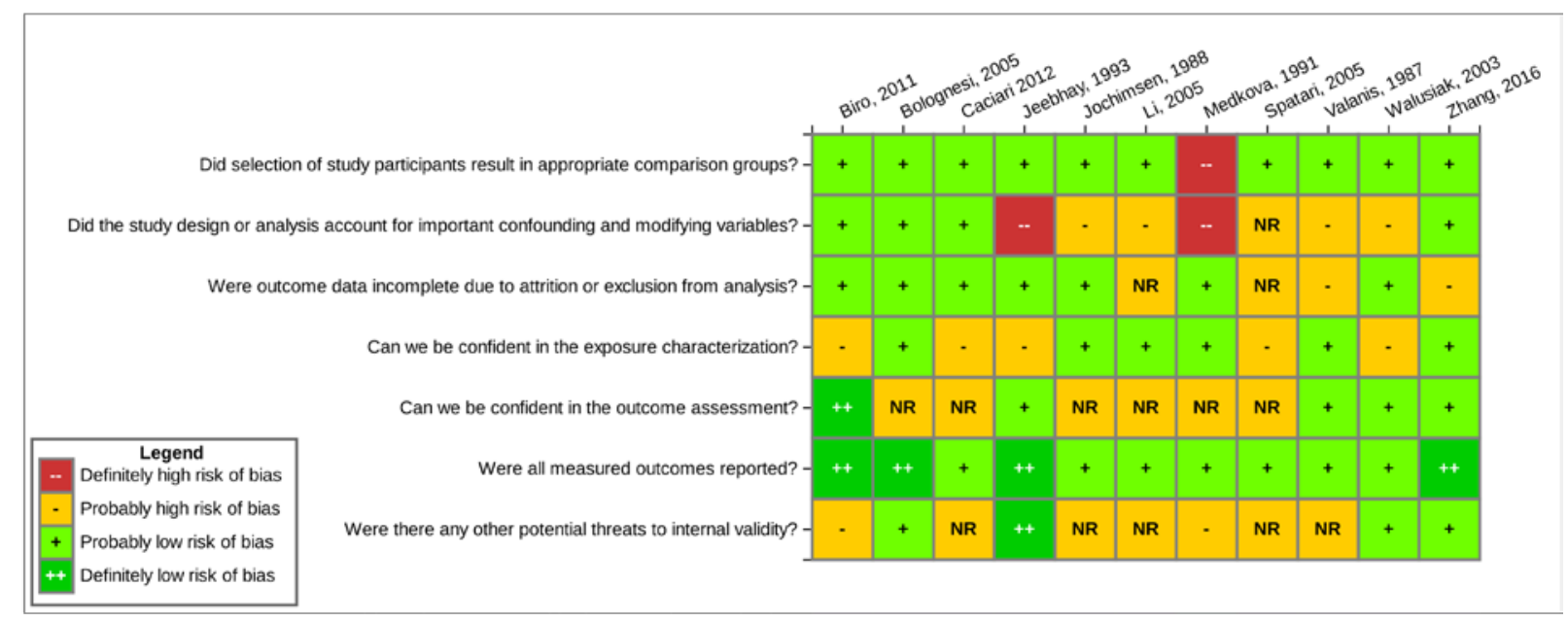

Figure E-6. Risk-of-bias Heat Map for Studies Reporting on Immune System Effects Associated with Occupational Exposure to Cancer Chemotherapy Agents

Interactive figure and additional study details in HAWC (NTP, 2015a).

\section{E.4. Kidney, Liver, and Cardiovascular System Toxicity}

The NTP evaluation identified six cross-sectional studies total that reported on kidney toxicity (five studies), liver toxicity (four studies), and cardiovascular system toxicity (two studies) (Caciari et al. 2012; Medková 1991a; Sessink et al. 1997a; Tigha Bouaziz et al. 2016; Walusiak et al. 2003; Zhang et al. 2016). The type of data available varied from biochemical measurements (e.g., secondary health outcomes) and the incidence of morbidity, which limited the ability to compare among studies.

Summary: Based on the available studies, there is very low confidence in the body of evidence that occupational exposure to cancer chemotherapy agents is associated with kidney, liver, or cardiovascular toxicity. There were very few studies per health outcome, which resulted in few effects evaluated across all studies (Table E-4). Another challenge was the lack of description provided for morbidity provided by the authors of one study (Medková 1991a). Of the four 
studies reporting on kidney toxicity, one study reported a higher rate of renal disease without further description and one of three studies reporting biochemical measurements reported a higher incidence of alterations in exposed subjects versus controls. In another example, one of two studies reported cardiovascular system toxicity. The final rating of very low confidence in the body of evidence reflects downgrading for serious risk-of-bias concerns across multiple key risk-of-bias questions (e.g., majority of studies were rated probably high risk of bias for confounding and exposure characterization) (Figure E-7 and Figure E-8). 
Table E-4. Studies Reporting on Kidney, Liver, or Cardiovascular System Toxicity

\begin{tabular}{|c|c|c|c|c|c|c|}
\hline Study & $\begin{array}{c}\text { Study Design } \\
\text { (Occupation, } \\
\text { Location, Years } \\
\text { Data Collected, Sex) }\end{array}$ & $\begin{array}{c}\text { No. of } \\
\text { Participants }\end{array}$ & Exposure Measure & $\begin{array}{c}\text { Outcome } \\
\text { Assessment }\end{array}$ & Analysis & $\begin{array}{c}\text { Results } \\
\text { (Outcomes Measured in } \\
\text { Bold) }\end{array}$ \\
\hline Caciari et al. (2012) & $\begin{array}{l}\text { Cross-sectional } \\
\text { (Healthcare workers } \\
\text { (doctors, nurses, social } \\
\text { and health assistants), } \\
\text { Italy, year NR, FM) }\end{array}$ & $\begin{array}{l}57 \text { exposed, } 57 \\
\text { unexposed }\end{array}$ & $\begin{array}{l}\text { Job title only (duration } \\
\text { NR) }\end{array}$ & Medical tests & $\begin{array}{l}\text { Student t-test, Chi- } \\
\text { square analysis }\end{array}$ & $\begin{array}{l}\text { Significantly higher levels of } \\
\text { bilirubin (possible indication of } \\
\text { liver damage) compared to } \\
\text { unexposed subjects; alanine or } \\
\text { aspartate aminotransferase, } \\
\text { alkaline phosphatase (measures } \\
\text { of liver function) NS; blood } \\
\text { urea nitrogen or creatinine } \\
\text { (measures of kidney function) } \\
\text { NS }\end{array}$ \\
\hline Medková (1991a) & $\begin{array}{l}\text { Cross-sectional } \\
\text { (Physicians, nurses, } \\
\text { auxiliary workers, } \\
\text { Czech Republic, year } \\
\text { NR, FM) }\end{array}$ & $\begin{array}{l}44 \text { exposed, } 32 \\
\text { unexposed partners } \\
\text { of exposed subjects }\end{array}$ & $\begin{array}{l}\text { Job title and } \\
\text { interviewer-led } \\
\text { questionnaire ( } \geq 5 \text { years, } \\
\mathrm{n}=25 ; 6-10 \text { years, } \\
\mathrm{n}=14 ; 11-15 \text { years, } \\
\mathrm{n}=4 ; \text { and } 16-20 \text { years, } \\
\mathrm{n}=1) \\
\text { Note: Exposed } \\
\text { personnel used gloves } \\
\text { and gowns; isolated } \\
\text { room for drug } \\
\text { preparation only used } \\
\text { by 25\% of exposed } \\
\text { subjects }\end{array}$ & $\begin{array}{l}\text { Interviewer-led } \\
\text { questionnaire }\end{array}$ & NR & $\begin{array}{l}\text { Higher occurrence of renal } \\
\text { disease and liver disease in } \\
\text { compared to unexposed } \\
\text { subjects, but statistics were not } \\
\text { provided; no difference in } \\
\text { occurrence of cardiovascular } \\
\text { morbidity (described as } \\
\text { diseases of the heart and } \\
\text { circulation) or hypertension }\end{array}$ \\
\hline Sessink et al. (1997a) & $\begin{array}{l}\text { Cross-sectional } \\
\text { (Nurses, lab technician, } \\
\text { and cleaning staff, t- } \\
\text { test, year NR, FM) }\end{array}$ & $\begin{array}{l}11 \text { exposed; } 23 \\
\text { unexposed nurses, } \\
\text { doctors, lab } \\
\text { technicians and a } \\
\text { cleaning woman }\end{array}$ & $\begin{array}{l}\text { Job title and [self- } \\
\text { reported] questionnaire } \\
\text { (13, range 3-25 years) } \\
\text { Note: Most exposed } \\
\text { personnel used PPE; } \\
\text { laminar down-flow } \\
\text { cabinets used for drug } \\
\text { preparation }\end{array}$ & Medical test & $\begin{array}{l}\text { Student's t-test, } \\
\text { Pearson's } \\
\text { correlation of log- } \\
\text { transformed data }\end{array}$ & $\begin{array}{l}\text { No difference in renal function } \\
\text { as measured by urinary albumin } \\
\text { and urinary retinol-binding } \\
\text { protein levels }\end{array}$ \\
\hline
\end{tabular}




\begin{tabular}{|c|c|c|c|c|c|c|}
\hline Study & $\begin{array}{c}\text { Study Design } \\
\text { (Occupation, } \\
\text { Location, Years } \\
\text { Data Collected, Sex) }\end{array}$ & $\begin{array}{c}\text { No. of } \\
\text { Participants }\end{array}$ & Exposure Measure & $\begin{array}{c}\text { Outcome } \\
\text { Assessment }\end{array}$ & Analysis & $\begin{array}{c}\text { Results } \\
\text { (Outcomes Measured in } \\
\text { Bold) }\end{array}$ \\
\hline $\begin{array}{l}\text { Tigha Bouaziz et al. } \\
\text { (2016) }\end{array}$ & $\begin{array}{l}\text { Cross-sectional } \\
\text { (Nurses, Algeria, year } \\
\text { NR, FM) }\end{array}$ & $\begin{array}{l}215 \text { unexposed, } 74 \\
\text { exposed }\end{array}$ & $\begin{array}{l}\text { Job title and [self- } \\
\text { reported] questionnaire } \\
(18.04 \pm 11.11 \text { years })\end{array}$ & Medical tests & $\begin{array}{l}\text { t-, z-, and Chi- } \\
\text { squared tests, } \\
\text { ANOVA, and } \\
\text { logistic regression } \\
\text { to adjust for age, } \\
\text { sex, work shift, and } \\
\text { other variables }\end{array}$ & $\begin{array}{l}\text { Significantly higher occurrence } \\
\text { cardiovascular morbidity } \\
\text { associated with oncology } \\
\text { nursing experience and higher } \\
\text { cytostatic contact index levels } \\
\text { (OR = 2.7, 95\% CI = } 1.26- \\
6.04) \text {; significantly higher } \\
\text { levels of creatinine, cholesterol, } \\
\text { and triglycerides compared to } \\
\text { unexposed nurses }\end{array}$ \\
\hline Walusiak et al. (2003) & $\begin{array}{l}\text { Cross-sectional } \\
\text { (Physicians, nurses, } \\
\text { orderlies, year NR, } \\
\text { Poland, F) }\end{array}$ & $\begin{array}{l}104 \text { exposed, } 103 \\
\text { unexposed }\end{array}$ & $\begin{array}{l}\text { Job title and self- } \\
\text { reported questionnaire } \\
(11.0 \pm 9.3 \text { years })\end{array}$ & $\begin{array}{l}\text { Self-report } \\
\text { questionnaire }\end{array}$ & $\begin{array}{l}\text { Mann-Whitney U- } \\
\text { test }\end{array}$ & $\begin{array}{l}\text { No difference in biochemical } \\
\text { measures of liver function } \\
\text { (aspartate and alanine } \\
\text { transaminases, bilirubin, } \\
\text { glutamyltransferase) or kidney } \\
\text { function (creatinine, urea) }\end{array}$ \\
\hline Zhang et al. (2016) & $\begin{array}{l}\text { Cross-sectional } \\
\text { (Nurses, China, } 2011 \\
\text { pre-PIVAS and } 2013 \\
\text { (post-PIVAS) in } \\
\text { Hospital A, F) }\end{array}$ & $\begin{array}{l}\text { Pre-PIVAS: } 138 \\
\text { unexposed, } 39 \\
\text { exposed non- } \\
\text { oncology, } 118 \\
\text { exposed oncology; } \\
\text { Post-PIVAS: } 185 \\
\text { unexposed, } 55 \\
\text { exposed non- } \\
\text { oncology, } 137 \\
\text { exposed oncology, } \\
\text { and } 163 \text { exposed } \\
\text { oncology nurses at } \\
\text { Hospital B) }\end{array}$ & $\begin{array}{l}\text { Job title and self- } \\
\text { reported questionnaire } \\
\text { ( } \geq 2 \text { years in current job) }\end{array}$ & $\begin{array}{l}\text { Self-report } \\
\text { questionnaire }\end{array}$ & $\begin{array}{l}\text { [Student] t-test, } \\
\text { Chi-square, and } \\
\text { Wilcoxon for } \\
\text { outcomes [authors } \\
\text { did not specify } \\
\text { which method] }\end{array}$ & $\begin{array}{l}\text { Significantly }(\mathrm{p}<0.05) \text { altered } \\
\text { levels of biochemical } \\
\text { measurements of liver function } \\
\text { (alanine transaminase, serum } \\
\text { aspartate transaminase) and } \\
\text { kidney function (creatinine, } \\
\text { blood urea nitrogen) compared } \\
\text { to unexposed nurses in Hospital } \\
\text { A regardless of PIVAS } \\
\text { implementation }\end{array}$ \\
\hline
\end{tabular}

ANOVA = analysis of variance, $\mathrm{CI}=$ confidence interval, $\mathrm{F}=$ female, $\mathrm{M}=$ male, No. = number, $\mathrm{NR}=$ not reported, $\mathrm{NS}=$ not significant, $\mathrm{OR}=$ odds ratio, $\mathrm{PIVAS}=$ pharmacy intravenous admixture service. 


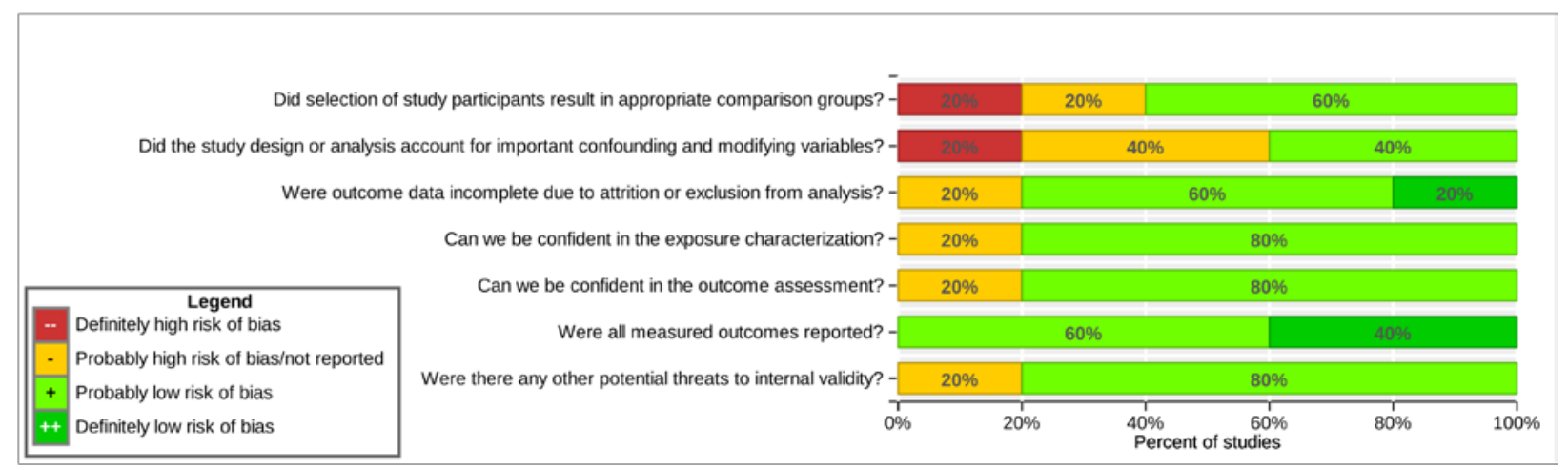

Figure E-7. Risk-of-bias Bar Graph for Studies Reporting on Kidney, Liver, or Cardiovascular System Toxicity Associated with Occupational Exposure to Cancer Chemotherapy Agents

Interactive figure and additional study details in HAWC (NTP, 2015a).

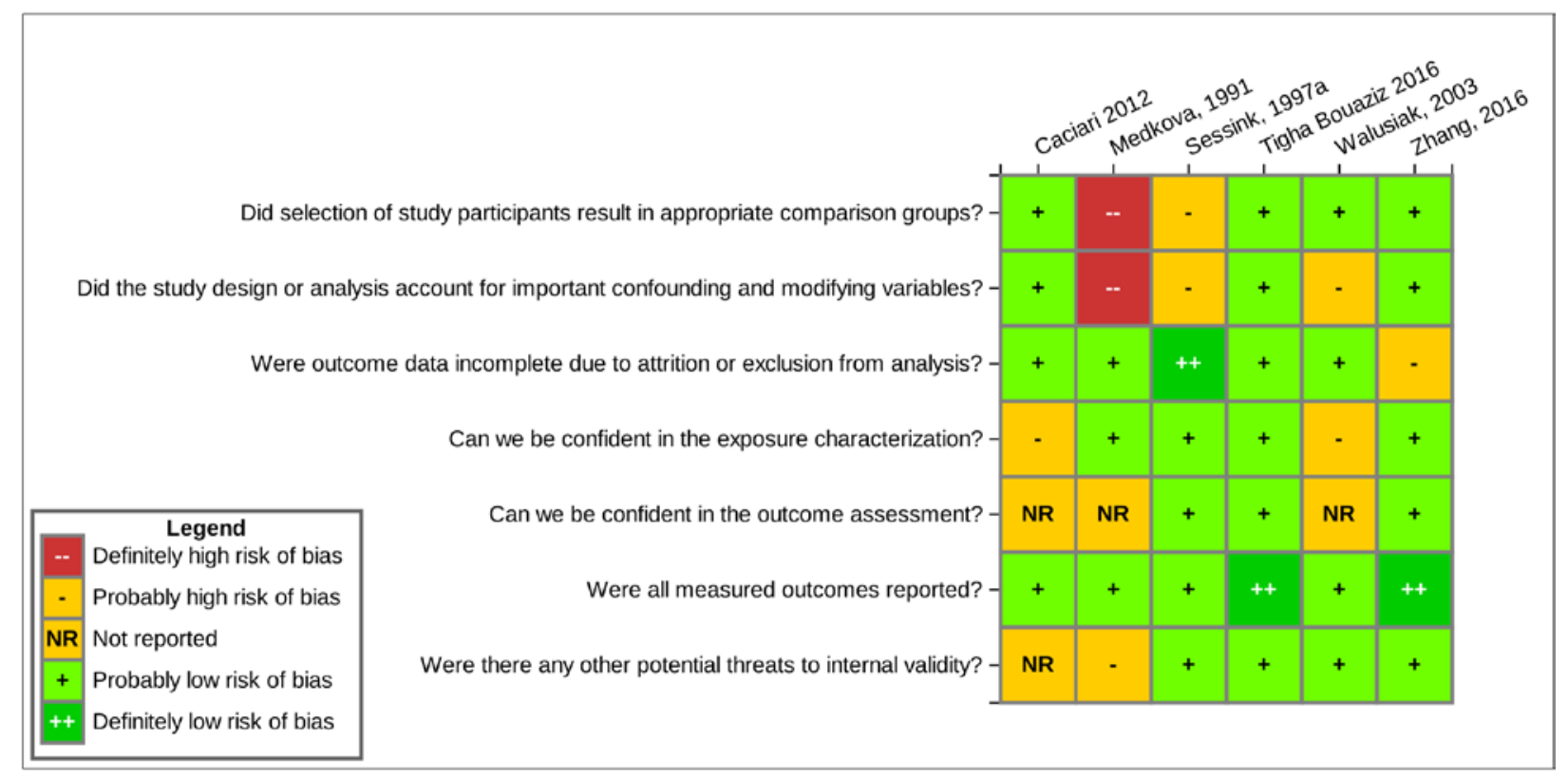

Figure E-8. Risk-of-bias Heat Map for Studies Reporting on Kidney, Liver, or Cardiovascular System Toxicity Associated with Occupational Exposure to Cancer Chemotherapy Agents

Interactive figure and additional study details in $\underline{\text { HAWC }}$ (NTP, 2015a).

\section{E.5. Reproductive Toxicity, Other Than Spontaneous Abortion}

In addition to spontaneous abortion, the NTP evaluation identified 24 studies of 19 unique populations reporting on reproductive and developmental toxicity, including pregnancy outcomes and female reproductive function (Bouyer et al. 1998; Elshamy et al. 2010; Fransman et al. 2007c; Hemminki et al. 1985; Krstev et al. 2003; Lawson et al. 2009; Lorente et al. 2000; McAbee et al. 1993; McDonald et al. 1988a; 1988b; Medková 1991a; Peelen et al. 1999; Ratner et al. 2010; Saurel-Cubizolles et al. 1993; Schaumburg and Olsen 1989; 1990a; 1991; Shortridge et al. 1995; Skov et al. 1992; Stücker et al. 1993; Valanis et al. 1997; Valanis et al. 1999; Walusiak et al. 2003; Zhang et al. 2016). The pregnancy outcomes included endpoints such as 
congenital malformations (12 studies), stillbirth (seven studies), preterm birth (seven studies), low birth weight or small for gestational age (eight studies), and ectopic pregnancy (three studies). Other studies reported on reproductive function, including time to pregnancy (three studies), infertility (three studies), and menstrual irregularities (three studies).

Summary: Based on the available data, there is very low confidence in the body of evidence that occupational exposure to cancer chemotherapy agents during pregnancy is associated with reproductive and developmental toxicity for endpoints other than spontaneous abortion.

Very few exposed cases were observed for some endpoints (e.g., congenital malformations), while there were very few studies were available for other endpoints (e.g., infertility) (Table E-5). There were 12 studies (of 11 unique populations) available that evaluated congenital malformations, including 11 studies evaluating any malformation (Elshamy et al. 2010; Fransman et al. 2007c; Hemminki et al. 1985; McAbee et al. 1993; McDonald et al. 1988a; Medková 1991a; Peelen et al. 1999; Ratner et al. 2010; Schaumburg and Olsen 1990a; Skov et al. 1992; Walusiak et al. 2003) and one study specifically evaluating oral clefts (Lorente et al. 2000). However, the results were inconsistent between studies with six of nine studies (one casecontrol study and five cross-sectional studies) reporting significantly higher rates of congenital malformations from exposed pregnancies, while three retrospective cohort studies, two with a larger number of exposed pregnancies, did not observe a significant increase. Only three studies limited the exposure characterization to the first trimester, and the results remained inconsistent when considering only the highest quality studies. Stillbirth did not appear to be associated with occupational exposure to cancer chemotherapy based on five available studies (one retrospective cohort study and six cross-sectional studies; six unique populations). However, the level of evidence for no effect conclusion requires a final rating of high confidence in the body of evidence (Rooney et al. 2014) and these studies did not achieve higher than a moderate confidence in the body of literature based on their study design. The final rating of very low confidence in the body of evidence for the reproductive and developmental toxicity effects, other than spontaneous abortion, reflected downgrading for inconsistency in the results for congenital malformations. No downgrading or upgrading for key risk-of-bias questions were identified for the other non-spontaneous abortion endpoints and few available studies were available for some endpoints, which also led to a final rating of very low confidence (Figure E-9 and Figure E-10). 
Table E-5. Studies Reporting on Reproductive and Developmental Toxicity, Other Than Spontaneous Abortions

\begin{tabular}{|c|c|c|c|c|c|c|}
\hline Study & $\begin{array}{l}\text { Study Design } \\
\text { (Occupation, } \\
\text { Location, Years } \\
\text { Data Collected, } \\
\text { Sex) }\end{array}$ & $\begin{array}{c}\text { No. of } \\
\text { Participants, } \\
\text { Pregnancies, or } \\
\text { Infants, as Noted }\end{array}$ & $\begin{array}{l}\text { Exposure } \\
\text { Measure }\end{array}$ & $\begin{array}{c}\text { Outcome } \\
\text { Assessment }\end{array}$ & Analysis & $\begin{array}{c}\text { Results } \\
\text { (Outcomes Measured in } \\
\text { Bold) }\end{array}$ \\
\hline Bouyer et al. (1998) & $\begin{array}{l}\text { Case-control } \\
\text { (Hospital personnel, } \\
\text { France, 1995, F) } \\
\text { Note: Follow-up } \\
\text { study to Saurel- } \\
\text { Cubizolles } 1993\end{array}$ & $\begin{array}{l}140 \text { cases, } 279 \\
\text { control pregnancies } \\
\text { (exposure to cancer } \\
\text { chemotherapy: } 37 \\
\text { pregnancies with past } \\
\text { exposure and } 43 \text { with } \\
\text { exposure in } 3 \text { months } \\
\text { prior to conception) }\end{array}$ & $\begin{array}{l}\text { Self-reported } \\
\text { questionnaire on past } \\
\text { and present } \\
\text { occupational } \\
\text { exposure to cancer } \\
\text { chemotherapy agents, } \\
\text { solvents, } \\
\text { disinfectants, } \\
\text { anesthetic agents, and } \\
\text { ionizing radiation } \\
\text { Note: Authors } \\
\text { excluded subjects } \\
\text { self-reporting to be } \\
\text { seldom exposed or } \\
\text { using a protective } \\
\text { device }\end{array}$ & $\begin{array}{l}\text { NR [presumably } \\
\text { medical records] }\end{array}$ & $\begin{array}{l}\text { Multivariate analysis } \\
\text { using unconditional } \\
\text { logistic regression }\end{array}$ & $\begin{array}{l}\text { No association with ectopic } \\
\text { pregnancy and past exposure } \\
\text { (adjOR }=1.56,95 \% \mathrm{CI}=0.65- \\
3.72,15 \text { exposed cases) or } 3 \text { - } \\
\text { month prior exposure } \\
\text { (adjOR }=0.95,95 \% \mathrm{CI}=0.39 \text { - } \\
2.31,10 \text { exposed cases) } \\
\text { compared to controls }\end{array}$ \\
\hline
\end{tabular}




\begin{tabular}{|c|c|c|c|c|c|c|}
\hline Study & $\begin{array}{l}\text { Study Design } \\
\text { (Occupation, } \\
\text { Location, Years } \\
\text { Data Collected, } \\
\text { Sex) }\end{array}$ & $\begin{array}{c}\text { No. of } \\
\text { Participants, } \\
\text { Pregnancies, or } \\
\text { Infants, as Noted }\end{array}$ & $\begin{array}{l}\text { Exposure } \\
\text { Measure }\end{array}$ & $\begin{array}{c}\text { Outcome } \\
\text { Assessment }\end{array}$ & Analysis & $\begin{array}{c}\text { Results } \\
\text { (Outcomes Measured in } \\
\text { Bold) }\end{array}$ \\
\hline Elshamy et al. (2010) & $\begin{array}{l}\text { Cross-sectional } \\
\text { (Nurses, Egypt, 2006, } \\
\text { F) }\end{array}$ & $\begin{array}{l}35 \text { exposed, } 29 \\
\text { unexposed }\end{array}$ & $\begin{array}{l}\text { Job title, length of } \\
\text { employment, self- } \\
\text { administered } \\
\text { questionnaire about } \\
\text { drug handling, } \\
\text { frequency of } \\
\text { handling, number of } \\
\text { accidents with cancer } \\
\text { chemotherapy in past } \\
\text { year, personal } \\
\text { protective equipment } \\
\text { usage, and level of } \\
\text { training. } \\
\text { Note: Only } 42.9 \% \text { of } \\
\text { nurses reported using } \\
\text { gloves when handling } \\
\text { patient waste, and } \\
45.7 \% \text { of nurses } \\
\text { admitted to eating in } \\
\text { the drug preparation } \\
\text { area }\end{array}$ & $\begin{array}{l}\text { Self-reported } \\
\text { questionnaire; no } \\
\text { definitions were } \\
\text { provided }\end{array}$ & $\begin{array}{l}\text { Chi-square test with } \\
\text { continuity correction, } \\
\text { if indicated }\end{array}$ & $\begin{array}{l}\text { Significantly higher ( }<0.05) \\
\text { percentage of exposed than } \\
\text { unexposed nurses experienced } \\
\text { infertility/subfertility }(11.4 \% \\
\text { vs. } 3.4 \%) \text {, premature labor } \\
(11.4 \% \text { vs. } 3.4 \%) \text {, fetal loss } \\
\text { [presumed stillbirth] ( } 8.6 \% \text { vs. } \\
3.4 \%) \text {, congenital } \\
\text { malformations ( } 8.6 \text { vs. } 3.4) \text {, } \\
\text { and developmental and } \\
\text { behavioral abnormalities of } \\
\text { offspring ( } 5.7 \% \text { vs. } 0 \%) \text {, and } \\
\text { higher but NS menstrual } \\
\text { changes and low birth weight }\end{array}$ \\
\hline
\end{tabular}




\begin{tabular}{|c|c|c|c|c|c|c|}
\hline Study & $\begin{array}{l}\text { Study Design } \\
\text { (Occupation, } \\
\text { Location, Years } \\
\text { Data Collected, } \\
\text { Sex) }\end{array}$ & $\begin{array}{c}\text { No. of } \\
\text { Participants, } \\
\text { Pregnancies, or } \\
\text { Infants, as Noted }\end{array}$ & $\begin{array}{l}\text { Exposure } \\
\text { Measure }\end{array}$ & $\begin{array}{c}\text { Outcome } \\
\text { Assessment }\end{array}$ & Analysis & $\begin{array}{c}\text { Results } \\
\text { (Outcomes Measured in } \\
\text { Bold) }\end{array}$ \\
\hline $\begin{array}{l}\text { Fransman et al. } \\
(2007 c)\end{array}$ & $\begin{array}{l}\text { Cross-sectional } \\
\text { (Nurses, the } \\
\text { Netherlands, 1990- } \\
\text { 1997, F) } \\
\text { Note: Same data as } \\
\text { Peelen } 1997\end{array}$ & $\begin{array}{l}\text { Exposed pregnancies: } \\
\text { 279 background, } 146 \\
\text { low, } 145 \text { medium, } \\
134 \text { high; } 555 \\
\text { unexposed } \\
\text { pregnancies }\end{array}$ & $\begin{array}{l}\text { Estimated dermal } \\
\text { exposure based on } \\
\text { job title, self-reported } \\
\text { questionnaire, and } \\
\text { glove usage in first } \\
\text { trimester of } \\
\text { pregnancy (or during } \\
\text { time trying to get } \\
\text { pregnant), combined } \\
\text { with data on CP glove } \\
\text { contamination } \\
\text { conducted in } 1996- \\
\text { 1997 and glove and } \\
\text { hand contamination } \\
\text { in } 2003-2007 \text {; } \\
\text { categorized as: } \\
\text { unexposed, } \\
\text { background, low } \\
\text { ( } \leq 0.20 \mu \mathrm{CP} / \mathrm{wk} \text { ), } \\
\text { medium ( }>0.20- \\
0.74 \mu \mathrm{CP} / \mathrm{wk}) \text { or } \\
\text { high exposure } \\
\text { ( }>0.74 \mu \mathrm{CP} / \mathrm{wk} \text { ) }\end{array}$ & $\begin{array}{l}\text { Self-reported } \\
\text { questionnaire }\end{array}$ & $\begin{array}{l}\text { Logistic regression } \\
\text { analysis; adjusted a } \\
\text { prior for age at } \\
\text { conception, parity, } \\
\text { smoking, alcohol } \\
\text { consumption, coffee } \\
\text { intake during 1st } \\
\text { trimester of } \\
\text { pregnancy, } \\
\text { vitamin/folic acid use } \\
\text { during pregnancy as } \\
\text { well as work factors } \\
\text { (e.g., co-exposures } \\
\text { during 1st trimester) }\end{array}$ & $\begin{array}{l}\text { Significantly longer time to } \\
\text { pregnancy in highly exposed } \\
\text { nurses compared to unexposed } \\
\text { nurses (aHR = } 0.8,95 \% \\
\mathrm{CI}=0.6-0.9 \text { ) and there was a } \\
\text { log-linear increase in low birth } \\
\text { weight per unit exposure } \\
(\mathrm{OR}=1.11,95 \% \mathrm{CI}=1.01- \\
1.21) \text { and premature birth per } \\
\text { unit exposure (OR = 1.08, 95\% } \\
\mathrm{CI}=1.00-1.17) \text {; data on } \\
\text { stillbirth; congenital } \\
\text { anomalies, and alterations in } \\
\text { sex ratio (\% boys) NS }\end{array}$ \\
\hline
\end{tabular}




\begin{tabular}{|c|c|c|c|c|c|c|}
\hline Study & $\begin{array}{l}\text { Study Design } \\
\text { (Occupation, } \\
\text { Location, Years } \\
\text { Data Collected, } \\
\text { Sex) } \\
\end{array}$ & $\begin{array}{c}\text { No. of } \\
\text { Participants, } \\
\text { Pregnancies, or } \\
\text { Infants, as Noted }\end{array}$ & $\begin{array}{l}\text { Exposure } \\
\text { Measure }\end{array}$ & $\begin{array}{c}\text { Outcome } \\
\text { Assessment }\end{array}$ & Analysis & $\begin{array}{c}\text { Results } \\
\text { (Outcomes Measured in } \\
\text { Bold) }\end{array}$ \\
\hline $\begin{array}{l}\text { Hemminki et al. } \\
\text { (1985) }\end{array}$ & $\begin{array}{l}\text { Case-control } \\
\text { (Nurses, 1973-1979, } \\
\text { Finland, F) }\end{array}$ & $\begin{array}{l}38 \text { cases and } 99 \\
\text { normal infants } \\
\text { (16 pregnancies } \\
\text { exposed to cancer } \\
\text { chemotherapy agents) }\end{array}$ & $\begin{array}{l}\text { Central Registry of } \\
\text { Health Care } \\
\text { Personnel and a } \\
\text { questionnaire to head } \\
\text { nurses regarding each } \\
\text { subjects' } \\
\text { occupational } \\
\text { exposures, including } \\
\text { anesthetic gases, } \\
\text { sterilizing agents, } \\
\text { disinfectants, cancer } \\
\text { chemotherapy drugs, } \\
\text { and x-ray radiation, } \\
\text { shift work and } \\
\text { telephone duty } \\
\text { Note: Exposed nurses } \\
\text { were from non- } \\
\text { oncology departments } \\
\text { where exposure was } \\
\text { relatively uncommon }\end{array}$ & Medical records & $\begin{array}{l}\text { Odds ratios and } \\
\text { logistic model of } \\
\text { conditional likelihood } \\
\text { function adjusted for } \\
\text { shift work and co- } \\
\text { exposures (e.g., } \\
\text { ionizing radiation) }\end{array}$ & $\begin{array}{l}\text { Significantly higher odds of } \\
\text { congenital malformations } \\
\text { compared to unexposed } \\
\text { pregnancies (adjOR }=4.7,95 \% \\
\mathrm{CI}=1.2-18.1 \text {, } 8 \text { cases) }\end{array}$ \\
\hline Krstev et al. (2003) & $\begin{array}{l}\text { Cross-sectional } \\
\text { (Nurses in } 8 \\
\text { hospitals, Serbia, year } \\
\text { NR, F) }\end{array}$ & $\begin{array}{l}186 \text { exposed, } 77 \\
\text { unexposed }\end{array}$ & $\begin{array}{l}\text { Job title and self- } \\
\text { reported } \\
\text { questionnaire; head } \\
\text { nurses reported on } \\
\text { work practice and } \\
\text { work conditions } \\
\text { Note: } 60.1 \text { to } 81.7 \% \\
\text { of exposed nurses } \\
\text { used gloves for drug } \\
\text { administration or } \\
\text { preparation; only one } \\
\text { hospital had a } \\
\text { vertical laminar } \\
\text { [flow] biological } \\
\text { safety cabinet, and } \\
\text { some concerns about } \\
\text { its maintenance }\end{array}$ & $\begin{array}{l}\text { Self-reported } \\
\text { questionnaire }\end{array}$ & $\begin{array}{l}\text { Relative risks using } \\
\text { two by two table } \\
\text { statistics. } \\
\text { Unconditional } \\
\text { logistic regression } \\
\text { measured effect of } \\
\text { age, smoking and } \\
\text { shift work }\end{array}$ & $\begin{array}{l}\text { No difference in the occurrence } \\
\text { of menstrual dysfunction } \\
\text { compared to unexposed nurses }\end{array}$ \\
\hline
\end{tabular}




\begin{tabular}{|c|c|c|c|c|c|c|}
\hline Study & $\begin{array}{l}\text { Study Design } \\
\text { (Occupation, } \\
\text { Location, Years } \\
\text { Data Collected, } \\
\text { Sex) }\end{array}$ & $\begin{array}{c}\text { No. of } \\
\text { Participants, } \\
\text { Pregnancies, or } \\
\text { Infants, as Noted }\end{array}$ & $\begin{array}{l}\text { Exposure } \\
\text { Measure }\end{array}$ & $\begin{array}{c}\text { Outcome } \\
\text { Assessment }\end{array}$ & Analysis & $\begin{array}{c}\text { Results } \\
\text { (Outcomes Measured in } \\
\text { Bold) }\end{array}$ \\
\hline Lawson et al. (2009) & $\begin{array}{l}\text { Cohort, retrospective } \\
\text { (Nurses' Health Study } \\
\text { II, USA, 1993-2001, } \\
\text { F) }\end{array}$ & $\begin{array}{l}270 \text { exposed, } \\
6,688 \text { unexposed } \\
\text { pregnancies, and } 19 \\
\text { pregnancies missing } \\
\text { exposure data }\end{array}$ & $\begin{array}{l}\text { Self-reported } \\
\text { questionnaire on first } \\
\text { trimester exposure to } \\
\text { anesthesia, cancer } \\
\text { chemotherapy agents, } \\
\text { anti-viral drugs, } \\
\text { sterilizing agents, and } \\
\text { X-ray radiation }\end{array}$ & $\begin{array}{l}\text { Self-report } \\
\text { questionnaire on most } \\
\text { recent pregnancy } \\
\text { since } 1993\end{array}$ & $\begin{array}{l}\text { Logistic regression } \\
\text { adjusted for age; } \\
\text { multivariate analysis } \\
\text { included all } 5 \text { work } \\
\text { exposures, parity, } \\
\text { shift work, and hours } \\
\text { worked adjusted for } \\
\text { age and parity }\end{array}$ & $\begin{array}{l}\text { No association with preterm } \\
\text { birth (only } 22 \text { exposed preterm } \\
\text { cases) }\end{array}$ \\
\hline Lorente et al. (2000) & $\begin{array}{l}\text { Case-control } \\
\text { (Working women, } \\
\text { Europe, 1989-1992, } \\
\text { F) }\end{array}$ & $\begin{array}{l}\text { Cases (infants): } 64 \\
\text { cleft lip with or } \\
\text { without cleft palate } \\
\text { and } 36 \text { cleft palate } \\
\text { only, } 751 \text { controls } \\
\text { (no. of infants } \\
\text { exposed to cancer } \\
\text { chemotherapy agents } \\
\text { not reported) }\end{array}$ & $\begin{array}{l}\text { Interviewer-led } \\
\text { questionnaire and } \\
\text { industrial hygienist } \\
\text { defined the exposure } \\
\text { by route of exposure, } \\
\text { level of exposure, } \\
\text { frequency, and } \\
\text { reliability of the } \\
\text { assessment } \\
\text { Note: Authors } \\
\text { reported that all } \\
\text { exposure to } \\
\text { antineoplastic drugs } \\
\text { was low-level, low- } \\
\text { frequency, and } \\
\text { certain exposure }\end{array}$ & $\begin{array}{l}\text { National } \\
\text { malformation registry }\end{array}$ & $\begin{array}{l}\text { Odds ratio adjusted } \\
\text { for study center, } \\
\text { maternal age, } \\
\text { mother's } \\
\text { socioeconomic status, } \\
\text { urbanization, and } \\
\text { country of origin; } \\
\text { backward stepwise } \\
\text { logistic regression to } \\
\text { determine exposures } \\
\text { independently } \\
\text { associated with oral } \\
\text { clefts }\end{array}$ & $\begin{array}{l}\text { Cleft palate was not } \\
\text { significantly elevated in cases } \\
\text { exposed to cancer } \\
\text { chemotherapy (adjOR = 5.1, } \\
\text { 95\% CI = 0.8-34, } 2 \text { exposed } \\
\text { cases) }\end{array}$ \\
\hline McAbee et al. (1993) & $\begin{array}{l}\text { Cross-sectional } \\
\text { (Nurses, USA, year } \\
\text { NR, F) }\end{array}$ & $\begin{array}{l}663 \text { women and } 1,133 \\
\text { pregnancies ( } 318 \\
\text { women exposed to } \\
\text { cancer chemotherapy } \\
\text { agents, no. of } \\
\text { exposed pregnancies } \\
\text { NR) }\end{array}$ & $\begin{array}{l}\text { Job title and self- } \\
\text { reported } \\
\text { questionnaire about } \\
\text { occupational } \\
\text { exposure to ionizing } \\
\text { radiation, cancer } \\
\text { chemotherapy agents, } \\
\text { solvents, and video } \\
\text { display terminals }\end{array}$ & $\begin{array}{l}\text { Self-report } \\
\text { questionnaire }\end{array}$ & $\begin{array}{l}\text { Mantel-Haenszel test } \\
\text { for linear associations }\end{array}$ & $\begin{array}{l}\text { Authors report a higher } \\
\text { prevalence of congenital } \\
\text { malformations in nurses } \\
\text { exposed to cancer } \\
\text { chemotherapy than unexposed } \\
\text { nurses ( } 10 \text { exposed cases, } \\
\mathrm{p}=0.02 \text { ) }\end{array}$ \\
\hline
\end{tabular}




\begin{tabular}{|c|c|c|c|c|c|c|}
\hline Study & $\begin{array}{l}\text { Study Design } \\
\text { (Occupation, } \\
\text { Location, Years } \\
\text { Data Collected, } \\
\text { Sex) }\end{array}$ & $\begin{array}{c}\text { No. of } \\
\text { Participants, } \\
\text { Pregnancies, or } \\
\text { Infants, as Noted }\end{array}$ & $\begin{array}{l}\text { Exposure } \\
\text { Measure }\end{array}$ & $\begin{array}{l}\text { Outcome } \\
\text { Assessment }\end{array}$ & Analysis & $\begin{array}{c}\text { Results } \\
\text { (Outcomes Measured in } \\
\text { Bold) }\end{array}$ \\
\hline $\begin{array}{l}\text { McDonald et al. } \\
\text { (1988a) }\end{array}$ & $\begin{array}{l}\text { Cross-sectional } \\
\text { (Working women, } \\
\text { Canada, 1982-1984, } \\
\text { F) } \\
\text { Note: Same } \\
\text { population as } \\
\text { McDonald } 1988 b\end{array}$ & $\begin{array}{l}\text { 47, } 913 \text { pregnancies } \\
\text { of working women } \\
\text { (152 pregnancies } \\
\text { exposed to cancer } \\
\text { chemotherapy agents) }\end{array}$ & $\begin{array}{l}\text { Interviewer-led } \\
\text { questionnaire; jobs } \\
\text { were assigned a code } \\
\text { without knowledge of } \\
\text { outcome; estimate } \\
\text { made from published } \\
\text { and unpublished } \\
\text { studies, and } \\
\text { occupational } \\
\text { surveillance } \\
\text { Note: Medical } \\
\text { questionnaire also } \\
\text { asked nurses or } \\
\text { physicians if they } \\
\text { administered cancer } \\
\text { chemotherapy agents } \\
\text { in the month after the } \\
\text { first missed menstrual } \\
\text { period }\end{array}$ & $\begin{array}{l}\text { Interviewer-led } \\
\text { questionnaire verified } \\
\text { by medical records of } \\
\text { previous pregnancy } \\
\text { outcome }\end{array}$ & $\begin{array}{l}\text { Poisson regression } \\
\text { evaluated observed } \\
\text { versus expected } \\
\text { cases; age, gravidity, } \\
\text { history of previous } \\
\text { abortion, ethnic } \\
\text { group, educational } \\
\text { level as well as } \\
\text { smoking habit and } \\
\text { alcohol consumption }\end{array}$ & $\begin{array}{l}\text { Significantly higher occurrence } \\
\text { of congenital malformations } \\
\text { from exposed pregnancies ( } 8 \\
\text { exposed cases observed versus } \\
4 \text { cases expected) }\end{array}$ \\
\hline $\begin{array}{l}\text { McDonald et al. } \\
\text { (1988b) }\end{array}$ & $\begin{array}{l}\text { Cross-sectional } \\
\text { (Working women, } \\
\text { 1982-1984, Canada, } \\
\text { F) } \\
\text { Note: Same } \\
\text { population as } \\
\text { McDonald 1988a }\end{array}$ & $\begin{array}{l}22,713 \text { ( } 63 \\
\text { pregnancies exposed } \\
\text { to cancer } \\
\text { chemotherapy agents) }\end{array}$ & $\begin{array}{l}\text { Interviewer-led } \\
\text { questionnaire; jobs } \\
\text { were assigned a code } \\
\text { without knowledge of } \\
\text { outcome; estimate } \\
\text { made from published } \\
\text { and unpublished } \\
\text { studies, and } \\
\text { occupational } \\
\text { surveillance }\end{array}$ & $\begin{array}{l}\text { Interviewer-led } \\
\text { questionnaire of } \\
\text { pregnancy outcome } \\
\text { prior to most recent } \\
\text { live birth }\end{array}$ & $\begin{array}{l}\text { Poisson regression } \\
\text { evaluated observed } \\
\text { versus expected } \\
\text { cases; age, gravidity, } \\
\text { history of previous } \\
\text { abortion, ethnic } \\
\text { group, educational } \\
\text { level, and smoking } \\
\text { habit and alcohol } \\
\text { consumption } \\
\end{array}$ & $\begin{array}{l}\text { None of the } 63 \text { exposed } \\
\text { pregnancies ended in stillbirth } \\
\text { (expected rate }=0.48 \text { ) }\end{array}$ \\
\hline Medková (1991a) & $\begin{array}{l}\text { Cross-sectional } \\
\text { (Physicians, nurses, } \\
\text { auxiliary workers, } \\
\text { Czech Republic, year } \\
\text { NR, FM) }\end{array}$ & $\begin{array}{l}50 \text { exposed infants, } \\
11 \text { unexposed infants } \\
\text { (to partners of } \\
\text { exposed subjects) }\end{array}$ & $\begin{array}{l}\text { Job title and } \\
\text { interviewer-led } \\
\text { questionnaire } \\
\text { Note: Isolated room } \\
\text { for drug preparation } \\
\text { only used by } 25 \% \text { of } \\
\text { exposed subjects; } \\
\text { glove and gowns used }\end{array}$ & $\begin{array}{l}\text { Interviewer-led } \\
\text { questionnaire }\end{array}$ & NR & $\begin{array}{l}\text { Two congenital } \\
\text { malformations were reported } \\
\text { in exposed infants compared to } \\
\text { none in the unexposed infants }\end{array}$ \\
\hline
\end{tabular}




\begin{tabular}{|c|c|c|c|c|c|c|}
\hline Study & $\begin{array}{l}\text { Study Design } \\
\text { (Occupation, } \\
\text { Location, Years } \\
\text { Data Collected, } \\
\text { Sex) }\end{array}$ & $\begin{array}{c}\text { No. of } \\
\text { Participants, } \\
\text { Pregnancies, or } \\
\text { Infants, as Noted }\end{array}$ & $\begin{array}{l}\text { Exposure } \\
\text { Measure }\end{array}$ & $\begin{array}{c}\text { Outcome } \\
\text { Assessment }\end{array}$ & Analysis & $\begin{array}{c}\text { Results } \\
\text { (Outcomes Measured in } \\
\text { Bold) }\end{array}$ \\
\hline Peelen et al. (1999) & $\begin{array}{l}\text { Cross-sectional } \\
\text { (Nurses, the } \\
\text { Netherlands, 1990- } \\
\text { 1997, F) } \\
\text { Note: Same data as } \\
\text { Fransman et al. } \\
\text { 2007c }\end{array}$ & $\begin{array}{l}\text { Exposed: } 249 \\
\text { pregnancies and } 229 \\
\text { live births; } \\
\text { unexposed: } 1,010 \\
\text { pregnancies and } 956 \\
\text { live births }\end{array}$ & $\begin{array}{l}\text { Job title, self-reported } \\
\text { questionnaire, } \\
\text { biomonitoring, and } \\
\text { environmental } \\
\text { monitoring }\end{array}$ & $\begin{array}{l}\text { Self-reported } \\
\text { questionnaire on most } \\
\text { recent pregnancies; } \\
\text { [same as Fransman et } \\
\text { al. 2007c] }\end{array}$ & $\begin{array}{l}\text { [Logistic regression]; } \\
\text { adjusted for age, } \\
\text { coffee consumption, } \\
\text { smoking, alcohol } \\
\text { consumption, } \\
\text { pregnancy, physical } \\
\text { strain, and co-- } \\
\text { exposures (e.g., } \\
\text { ionizing radiation, } \\
\text { disinfectants), as } \\
\text { needed }\end{array}$ & $\begin{array}{l}\text { Significantly higher odds of } \\
\text { congenital malformations } \\
\text { (OR = 5.1, 95\% CI 1.1- } \\
23.6 \text {; > } 7 \text { exposed cases), and } \\
\text { low birth weight in infants of } \\
\text { exposed nurses preparing the } \\
\text { agents (adjOR = } 16.7,95 \% \\
\mathrm{CI}=3.4-81.6 \text { ) and low birth } \\
\text { weight in infants of exposed } \\
\text { nurses involved in cleaning } \\
\text { activities associated with the } \\
\text { agents (adjOR = 2.9, 95\% } \\
\text { CI = 1.2-7.2) compared with } \\
\text { unexposed nurses; time to } \\
\text { pregnancy, stillbirth, } \\
\text { premature birth NS }\end{array}$ \\
\hline
\end{tabular}




\begin{tabular}{|c|c|c|c|c|c|c|}
\hline Study & $\begin{array}{l}\text { Study Design } \\
\text { (Occupation, } \\
\text { Location, Years } \\
\text { Data Collected, } \\
\text { Sex) }\end{array}$ & $\begin{array}{c}\text { No. of } \\
\text { Participants, } \\
\text { Pregnancies, or } \\
\text { Infants, as Noted }\end{array}$ & $\begin{array}{l}\text { Exposure } \\
\text { Measure }\end{array}$ & $\begin{array}{c}\text { Outcome } \\
\text { Assessment }\end{array}$ & Analysis & $\begin{array}{c}\text { Results } \\
\text { (Outcomes Measured in } \\
\text { Bold) }\end{array}$ \\
\hline Ratner et al. (2010) & $\begin{array}{l}\text { Cohort, retrospective } \\
\text { (Female registered } \\
\text { nurses from British } \\
\text { Columbia, Canada, } \\
\text { 1974-2000, F) } \\
\text { Note: Subjects } \\
\text { followed until } 2000\end{array}$ & $\begin{array}{l}22,491 \text { infants total: } \\
190 \text { exposed (Method } \\
1 \text {, ever working in } \\
\text { oncology or a cancer } \\
\text { center) or } \\
1,062 \\
\text { exposed } \geq 15 \text { days } \\
\text { during } 1 \text { st trimester } \\
\text { or } 2,650 \text { during } \\
10 \text { years prior to } \\
\text { pregnancy (Method } 2 \text {, } \\
\text { weighted duration of } \\
\text { exposure) }\end{array}$ & $\begin{array}{l}\text { Two exposure } \\
\text { measures: } \\
\text { Method } 1 \text { : Years } \\
\text { employed in } \\
\text { oncology or at a } \\
\text { cancer center (never, } \\
<5 \text { years, } \geq 5 \text { years, or } \\
\text { ever) and Method 2: } \\
\text { weighted duration of } \\
\text { exposure based on } \\
\text { job responsibilities } \\
\text { ( }<15 \text { days, } \geq 15 \text { to } \\
60 \text { days, and } \\
>60 \text { days, or } \\
\geq 15 \text { days; } 15 \text { days } \\
\text { equivalent to } 1 \text { year } \\
\text { of work in a job } \\
\text { likely not exposed } \\
\text { and } 60 \text { days } \\
\text { equivalent to } 1 \text { year } \\
\text { of work in job likely } \\
\text { exposed to cancer } \\
\text { chemotherapy agents) }\end{array}$ & $\begin{array}{l}\text { Provincial cancer } \\
\text { registry }\end{array}$ & $\begin{array}{l}\text { Logistic regression; } \\
\text { adjusted for age of } \\
\text { mother at birth, year } \\
\text { of birth, and sex of } \\
\text { child }\end{array}$ & $\begin{array}{l}\text { Not significant, but higher, odds } \\
\text { of congenital malformations } \\
\text { (Method 1, adjOR = 1.42, 95\% } \\
\text { CI = 0.86-2.36, } 17 \text { exposed } \\
\text { cases), premature birth } \\
\text { (Method 1, adjOR = 1.88, 95\% } \\
\mathrm{CI}=0.80-4.41,6 \text { exposed } \\
\text { cases), and low birth weight } \\
\text { (Method 1, adjOR = 1.41, 95\% } \\
\mathrm{CI}=0.44-4.54,3 \text { exposed } \\
\text { cases) following exposure at } \\
\text { any time during pregnancy; } \\
\text { using Method 2, congenital } \\
\text { malformations (e.g., cleft } \\
\text { palate/lip, adjOR = 1.84, 95\% } \\
\text { CI = 0.75-4.49, } 6 \text { exposed } \\
\text { cases), premature birth, low } \\
\text { birth weight, and stillbirth } \\
\text { were NS }\end{array}$ \\
\hline $\begin{array}{l}\text { Saurel-Cubizolles et } \\
\text { al. (1993) }\end{array}$ & $\begin{array}{l}\text { Cross-sectional } \\
\text { (Medical personnel } \\
\text { except physicians, } \\
\text { France, 1987-1988, } \\
\text { F) }\end{array}$ & $\begin{array}{l}82 \text { exposed, } 599 \\
\text { unexposed } \\
\text { pregnancies }\end{array}$ & $\begin{array}{l}\text { Job title and } \\
\text { interviewer-led } \\
\text { questionnaire asked } \\
\text { about exposure to } \\
\text { anesthetic gases, } \\
\text { formol, ionizing } \\
\text { radiation, or } \\
\text { antineoplastic drugs } \\
\text { during the first } \\
\text { trimester of } \\
\text { pregnancy }\end{array}$ & $\begin{array}{l}\text { Interviewer-led } \\
\text { questionnaire }\end{array}$ & $\begin{array}{l}\text { Chi-square analysis } \\
\text { and logistic } \\
\text { regression }\end{array}$ & $\begin{array}{l}\text { Significantly greater odds of } \\
\text { ectopic pregnancy in medical } \\
\text { personnel exposed during first } \\
\text { trimester to cancer } \\
\text { chemotherapy agents } \\
\text { (adjOR }=11.4,95 \% \mathrm{CI}=2.7- \\
47.6,6 \text { exposed cases) } \\
\text { compared to unexposed } \\
\text { subjects }\end{array}$ \\
\hline
\end{tabular}




\begin{tabular}{|c|c|c|c|c|c|c|}
\hline Study & $\begin{array}{l}\text { Study Design } \\
\text { (Occupation, } \\
\text { Location, Years } \\
\text { Data Collected, } \\
\text { Sex) }\end{array}$ & $\begin{array}{c}\text { No. of } \\
\text { Participants, } \\
\text { Pregnancies, or } \\
\text { Infants, as Noted }\end{array}$ & $\begin{array}{l}\text { Exposure } \\
\text { Measure }\end{array}$ & $\begin{array}{c}\text { Outcome } \\
\text { Assessment }\end{array}$ & Analysis & $\begin{array}{c}\text { Results } \\
\text { (Outcomes Measured in } \\
\text { Bold) }\end{array}$ \\
\hline $\begin{array}{l}\text { Schaumburg and } \\
\text { Olsen (1989) }\end{array}$ & $\begin{array}{l}\text { Cohort, retrospective } \\
\text { (Pharmacy assistants, } \\
\text { Denmark, 1979- } \\
\text { 1984, F) } \\
\text { Note: Same } \\
\text { population as } \\
\text { Schaumburg and } \\
\text { Olsen 1990a; } 1991\end{array}$ & $\begin{array}{l}4.924 \text { women; } 1,124 \\
\text { unexposed; only } 8 \\
\text { exposed to cancer } \\
\text { chemotherapy agents }\end{array}$ & $\begin{array}{l}\text { Membership in } \\
\text { national union of } \\
\text { pharmacy assistants } \\
\text { and self-reported } \\
\text { questionnaire asked } \\
\text { about work during } \\
\text { pregnancy, type of } \\
\text { pharmacy, work } \\
\text { tasks, exposure to } \\
\text { drugs and chemicals }\end{array}$ & Medical records & $\begin{array}{l}\text { Stratified analyses } \\
\text { with the Mantel- } \\
\text { Haenszel test } \\
\text { statistics and an } \\
\text { unconditional logistic } \\
\text { regression model; } \\
\text { adjusted for parity, } \\
\text { year of graduation, } \\
\text { smoking, and alcohol } \\
\text { consumption }\end{array}$ & $\begin{array}{l}\text { Time to pregnancy was not } \\
\text { prolonged for the } 8 \text { exposed } \\
\text { subjects; all women became } \\
\text { pregnancy in } 0-6 \text { months }\end{array}$ \\
\hline $\begin{array}{l}\text { Schaumburg and } \\
\text { Olsen (1990a) }\end{array}$ & $\begin{array}{l}\text { Cohort, retrospective } \\
\text { (Pharmacy assistants, } \\
\text { Denmark, 1979- } \\
\text { 1984, F) } \\
\text { Note: Same } \\
\text { population as } \\
\text { Schaumburg and } \\
\text { Olsen 1989; } 1991\end{array}$ & $\begin{array}{l}\text { 1,182 births; } 718 \\
\text { unexposed } \\
\text { pregnancies; only } 8 \\
\text { pregnancies exposed } \\
\text { to cancer } \\
\text { chemotherapy agents }\end{array}$ & $\begin{array}{l}\text { Membership in } \\
\text { national union of } \\
\text { pharmacy assistants } \\
\text { and self-reported } \\
\text { questionnaire asked } \\
\text { about work during } \\
\text { pregnancy, type of } \\
\text { pharmacy, work } \\
\text { tasks, exposure to } \\
\text { drugs and chemicals }\end{array}$ & $\begin{array}{l}\text { Medical records and } \\
\text { Central Bureau of } \\
\text { Statistics }\end{array}$ & $\begin{array}{l}\text { Unconditional } \\
\text { logistic regression } \\
\text { model; adjusted for } \\
\text { maternal age, parity, } \\
\text { smoking }\end{array}$ & $\begin{array}{l}\text { No congenital malformations } \\
\text { were observed in offspring of } 8 \\
\text { exposed pregnancies }\end{array}$ \\
\hline $\begin{array}{l}\text { Schaumburg and } \\
\text { Olsen (1991) }\end{array}$ & $\begin{array}{l}\text { Cohort, retrospective } \\
\text { (Pharmacy assistants, } \\
\text { Denmark, 1979- } \\
\text { 1984, F) } \\
\text { Note: Same } \\
\text { population as } \\
\text { Schaumburg and } \\
\text { Olsen 1989; 1990a }\end{array}$ & $\begin{array}{l}\text { 1,182 births; } 709 \\
\text { unexposed } \\
\text { pregnancies; only } 8 \\
\text { pregnancies exposed } \\
\text { to cancer } \\
\text { chemotherapy agents }\end{array}$ & $\begin{array}{l}\text { Membership in } \\
\text { national union of } \\
\text { pharmacy assistants } \\
\text { and self-reported } \\
\text { questionnaire asked } \\
\text { about work during } \\
\text { pregnancy, type of } \\
\text { pharmacy, work } \\
\text { tasks, exposure to } \\
\text { drugs and chemicals }\end{array}$ & $\begin{array}{l}\text { Medical records and } \\
\text { Central Bureau of } \\
\text { Statistics }\end{array}$ & $\begin{array}{l}\text { Analysis of variance } \\
\text { and unconditional } \\
\text { logistic regression } \\
\text { model; adjusted for } \\
\text { maternal age, parity, } \\
\text { smoking, and alcohol } \\
\text { consumption }\end{array}$ & $\begin{array}{l}\text { Non-significant decreases in } \\
\text { birth weight in offspring of } \\
\text { exposed subjects; no increased } \\
\text { risk of premature birth } \\
\text { (OR = } 6.50,95 \% \text { CI }=0.9 \text { - } \\
31.6: 2 \text { of } 8 \text { exposed births were } \\
\text { premature) }\end{array}$ \\
\hline
\end{tabular}




\begin{tabular}{|c|c|c|c|c|c|c|}
\hline Study & $\begin{array}{l}\text { Study Design } \\
\text { (Occupation, } \\
\text { Location, Years } \\
\text { Data Collected, } \\
\text { Sex) }\end{array}$ & $\begin{array}{c}\text { No. of } \\
\text { Participants, } \\
\text { Pregnancies, or } \\
\text { Infants, as Noted }\end{array}$ & $\begin{array}{l}\text { Exposure } \\
\text { Measure }\end{array}$ & $\begin{array}{c}\text { Outcome } \\
\text { Assessment }\end{array}$ & Analysis & $\begin{array}{c}\text { Results } \\
\text { (Outcomes Measured in } \\
\text { Bold) }\end{array}$ \\
\hline $\begin{array}{l}\text { Shortridge et al. } \\
\text { (1995) }\end{array}$ & $\begin{array}{l}\text { Prospective cohort } \\
\text { (Nurses in Oncology } \\
\text { Nursing Society, } \\
\text { USA, F) }\end{array}$ & $\begin{array}{l}596 \text { exposed, } 497 \\
\text { unexposed women }\end{array}$ & $\begin{array}{l}\text { Self-administered, } \\
\text { validated } \\
\text { questionnaire }\end{array}$ & $\begin{array}{l}\text { Self-reported } \\
\text { questionnaire }\end{array}$ & $\begin{array}{l}\text { Logistic regression; } \\
\text { evaluated age, } \\
\text { interaction of age and } \\
\text { exposure, and stress }\end{array}$ & $\begin{array}{l}\text { Significant logistic regression } \\
\text { results for menstrual } \\
\text { dysfunction included: age } \\
\text { groups }>30 \text { years old } \\
(\mathrm{p}=0.002) \text {, the interaction of } \\
\text { age group and exposure } \\
(\mathrm{p}=0.03) \text {, and job stress } \\
(\mathrm{p}=0.045) \text {; menstrual } \\
\text { dysfunction was significantly } \\
\text { higher in nurses ages } \geq 30 \text { years } \\
\text { old }(\mathrm{OR}=1.6,95 \% \mathrm{CI}=1.3- \\
1.9) \text { and }>46 \text { years old } \\
(\mathrm{OR}=1.4,95 \% \mathrm{CI}=1.1-1.8)\end{array}$ \\
\hline Skov et al. (1992) & $\begin{array}{l}\text { Cohort, retrospective } \\
\text { (Nurses, Denmark, } \\
\text { 1973-1988, F) }\end{array}$ & $\begin{array}{l}213 \text { exposed, } 604 \\
\text { unexposed } \\
\text { pregnancies }\end{array}$ & $\begin{array}{l}\text { Employment records } \\
\text { of } 5 \text { oncology } \\
\text { hospitals, and data } \\
\text { from head nurses } \\
\text { regarding drug } \\
\text { handling and } \\
\text { administration of } \\
\text { individual subjects of } \\
\text { individual subjects }\end{array}$ & Medical records & $\begin{array}{l}\text { Logistic regression } \\
\text { analysis; adjustment } \\
\text { for maternal age }\end{array}$ & $\begin{array}{l}\text { No higher odds of congenital } \\
\text { malformations (adjOR }=0.99 \text {, } \\
95 \% \mathrm{CI}=0.5-1.96 \text { ) or low } \\
\text { birth weight (adjOR }=1.06 \text {, } \\
95 \% \mathrm{CI}=0.42-2.67 \text { ), and no } \\
\text { higher odds of altered sex ratio } \\
\text { (\% male) (OR }=1.12,95 \% \\
\mathrm{CI}=0.79-1.61 \text { ) compared to } \\
\text { unexposed infants; no } \\
\text { difference in gestational age at } \\
\text { birth }\end{array}$ \\
\hline Stücker et al. (1993) & $\begin{array}{l}\text { Cross-sectional } \\
\text { (Nurses, France, } \\
\text { 1985-1986, F) }\end{array}$ & $\begin{array}{l}107 \text { exposed, } 292 \\
\text { unexposed } \\
\text { pregnancies }\end{array}$ & $\begin{array}{l}\text { Job position and } \\
\text { interviewer-led } \\
\text { questionnaire } \\
\text { Note: At time of } \\
\text { study, no special } \\
\text { protective equipment } \\
\text { was in operation and } \\
\text { use of PPE was not } \\
\text { uniform }\end{array}$ & $\begin{array}{l}\text { Interviewer-led } \\
\text { questionnaire }\end{array}$ & $\begin{array}{l}\text { Logistic regression } \\
\text { analysis; evaluated } \\
\text { effect of gestational } \\
\text { age, infant sex, age of } \\
\text { mother at birth, } \\
\text { parity, BMI maternal } \\
\text { smoking during } \\
\text { pregnancy }\end{array}$ & $\begin{array}{l}\text { No association of birth weight } \\
\text { with exposure to cancer } \\
\text { chemotherapy agents during } \\
\text { pregnancy }\end{array}$ \\
\hline
\end{tabular}




\begin{tabular}{|c|c|c|c|c|c|c|}
\hline Study & $\begin{array}{l}\text { Study Design } \\
\text { (Occupation, } \\
\text { Location, Years } \\
\text { Data Collected, } \\
\text { Sex) }\end{array}$ & $\begin{array}{c}\text { No. of } \\
\text { Participants, } \\
\text { Pregnancies, or } \\
\text { Infants, as Noted }\end{array}$ & $\begin{array}{l}\text { Exposure } \\
\text { Measure }\end{array}$ & $\begin{array}{c}\text { Outcome } \\
\text { Assessment }\end{array}$ & Analysis & $\begin{array}{c}\text { Results } \\
\text { (Outcomes Measured in } \\
\text { Bold) }\end{array}$ \\
\hline Valanis et al. (1997) & $\begin{array}{l}\text { Case-control } \\
\text { (Nurses, nurse's } \\
\text { aides, pharmacists, } \\
\text { and pharmacy } \\
\text { technicians, USA, } \\
\text { 1988-1989, FM) } \\
\text { Note: Same } \\
\text { population as Valanis } \\
1999\end{array}$ & $\begin{array}{l}405 \text { cases and } 1,125 \\
\text { controls ( } 355 \text { exposed } \\
\text { women due to prior } \\
\text { handling of cancer } \\
\text { chemotherapy agents) }\end{array}$ & $\begin{array}{l}\text { Self-reported, } \\
\text { validated } \\
\text { questionnaire }\end{array}$ & $\begin{array}{l}\text { Self-reported } \\
\text { questionnaire }\end{array}$ & $\begin{array}{l}\text { Two-way ANOVA } \\
\text { and conditional } \\
\text { logistic regression }\end{array}$ & $\begin{array}{l}\text { Significant association of } \\
\text { infertility with prior handling } \\
\text { of cancer chemotherapy agents } \\
\text { regardless of skin contact } \\
\text { compared to unexposed } \\
\text { subjects (OR }=1.5,95 \% \text { CI } \\
1.2-2,206 \text { exposed cases) }\end{array}$ \\
\hline Valanis et al. (1999) & $\begin{array}{l}\text { Cross-sectional } \\
\text { (Nurses, nurse's } \\
\text { aides, pharmacists, } \\
\text { and pharmacy } \\
\text { technicians, USA, } \\
\text { 1988-1989, FM) } \\
\text { Note: Same } \\
\text { population as Valanis } \\
1997\end{array}$ & $\begin{array}{l}\text { 1,448 exposed, } 5,297 \\
\text { unexposed } \\
\text { pregnancies ( } 6,041 \\
\text { pregnancies of female } \\
\text { personnel and } 704 \\
\text { from spouse of male } \\
\text { personnel) }\end{array}$ & $\begin{array}{l}\text { Self-reported, } \\
\text { validated } \\
\text { questionnaire; } \\
\text { subjects exposed to } \\
\text { cancer chemotherapy } \\
\text { agents } 2 \text { years prior } \\
\text { or during pregnancy }\end{array}$ & $\begin{array}{l}\text { Self-report } \\
\text { questionnaire }\end{array}$ & $\begin{array}{l}\text { Logistic regression } \\
\text { analyses for (1) } \\
\text { independence model } \\
\text { and ( } 2 \text { ) generalized } \\
\text { estimating equations } \\
\text { model; all models } \\
\text { adjusted for age and } \\
\text { gravidity at time of } \\
\text { pregnancy, maternal } \\
\text { smoking during } \\
\text { pregnancy }\end{array}$ & $\begin{array}{l}\text { No difference in stillbirth in } \\
\text { subjects exposed during } \\
\text { pregnancy (no adjustment for } \\
\text { prior exposure) compared to } \\
\text { unexposed subjects } \\
\text { (adjOR }=1.1,95 \% \text { CI }=0.5- \\
2.2 \text { ) }\end{array}$ \\
\hline Walusiak et al. (2003) & $\begin{array}{l}\text { Cross-sectional } \\
\text { (Physicians, nurses, } \\
\text { orderlies, year NR, } \\
\text { Poland, F) }\end{array}$ & $\begin{array}{l}84 \text { exposed, } 169 \\
\text { unexposed } \\
\text { pregnancies }\end{array}$ & $\begin{array}{l}\text { Job title and self- } \\
\text { reported } \\
\text { questionnaire }\end{array}$ & $\begin{array}{l}\text { Self-report } \\
\text { questionnaire }\end{array}$ & Mann-Whitney U-test & $\begin{array}{l}\text { Significantly higher occurrence } \\
\text { of congenital malformations } \\
\text { in exposed ( } 4.8 \% \text { ) versus } \\
\text { unexposed pregnancies ( } 0.6 \%) \text {; } \\
\text { [but incidence did not appear to } \\
\text { be higher that US general } \\
\text { population value of } 3.0 \% \\
\text { (Correa et al. 2007)]; data on } \\
\text { ectopic pregnancy, premature } \\
\text { birth, and birth weight NS }\end{array}$ \\
\hline
\end{tabular}




\begin{tabular}{|c|c|c|c|c|c|c|}
\hline Study & $\begin{array}{l}\text { Study Design } \\
\text { (Occupation, } \\
\text { Location, Years } \\
\text { Data Collected, } \\
\text { Sex) }\end{array}$ & $\begin{array}{c}\text { No. of } \\
\text { Participants, } \\
\text { Pregnancies, or } \\
\text { Infants, as Noted }\end{array}$ & $\begin{array}{l}\text { Exposure } \\
\text { Measure }\end{array}$ & $\begin{array}{c}\text { Outcome } \\
\text { Assessment }\end{array}$ & Analysis & $\begin{array}{c}\text { Results } \\
\text { (Outcomes Measured in } \\
\text { Bold) }\end{array}$ \\
\hline Zhang et al. (2016) & $\begin{array}{l}\text { Cross-sectional } \\
\text { (Nurses, China, } 2011 \\
\text { pre-PIVAS and } 2013 \\
\text { (post-PIVAS) in } \\
\text { Hospital A, F) }\end{array}$ & $\begin{array}{l}\text { Pre-PIVAS: } 106 \\
\text { unexposed, } 30 \\
\text { exposed non- } \\
\text { oncology, } 82 \text { exposed } \\
\text { oncology; Post- } \\
\text { PIVAS: } 108 \\
\text { unexposed, } 29 \\
\text { exposed non- } \\
\text { oncology, } 87 \text { exposed } \\
\text { oncology, and } 112 \\
\text { exposed oncology } \\
\text { nurses at Hospital B) }\end{array}$ & $\begin{array}{l}\text { Job title and self- } \\
\text { reported } \\
\text { questionnaire } \\
\text { Note: All exposed } \\
\text { nurses involved in } \\
\text { mixing and/or } \\
\text { administration of } \\
\text { cancer chemotherapy } \\
\text { agents }\end{array}$ & $\begin{array}{l}\text { Self-report } \\
\text { questionnaire }\end{array}$ & $\begin{array}{l}\text { [Student] t-test, Chi- } \\
\text { square, and Wilcoxon } \\
\text { for outcomes [authors } \\
\text { did not specify which } \\
\text { method] }\end{array}$ & $\begin{array}{l}\text { Significantly higher rate of } \\
\text { infertility among exposed } \\
\text { oncology nurses versus } \\
\text { unexposed nurses in Hospital A } \\
\text { both before ( }<<0.01 ; 26.8 \\
\text { versus } 9.4 \% \text { ) and after } \\
\text { implementation of the PIVAS } \\
\text { ( }<<0.05 ; 13.8 \% \text { versus } 3.7 \% \text { ); } \\
\text { significant ( }<0.05 \text { ) decrease in } \\
\text { infertility ( } 13.8 \% \text { versus } 26.8 \% \text { ) } \\
\text { and increase in full-term births } \\
\text { (reduction in premature } \\
\text { births) (33.3\% versus } 15.9 \%) \\
\text { in exposed oncology nurses } \\
\text { after implementation of PIVAS } \\
\text { compared to pre-PIVAS; } \\
\text { significantly higher rates of } \\
\text { infertility ( }<<0.01 \text {; } 30.4 \% \\
\text { versus } 13.8 \% \text { ) and lower full- } \\
\text { term births (p<0.05; } 19.6 \% \\
\text { versus } 33.3 \% \text { ) were observed in } \\
\text { Hospital B (no PIVAS) } \\
\text { compared to post-PIVAS } \\
\text { Hospital A }\end{array}$ \\
\hline
\end{tabular}

$\mathrm{NR}=$ not reported, NS = not significant OR = odds ratio, PIVAS = pharmacy intravenous admixture service, USA = United States of America. 


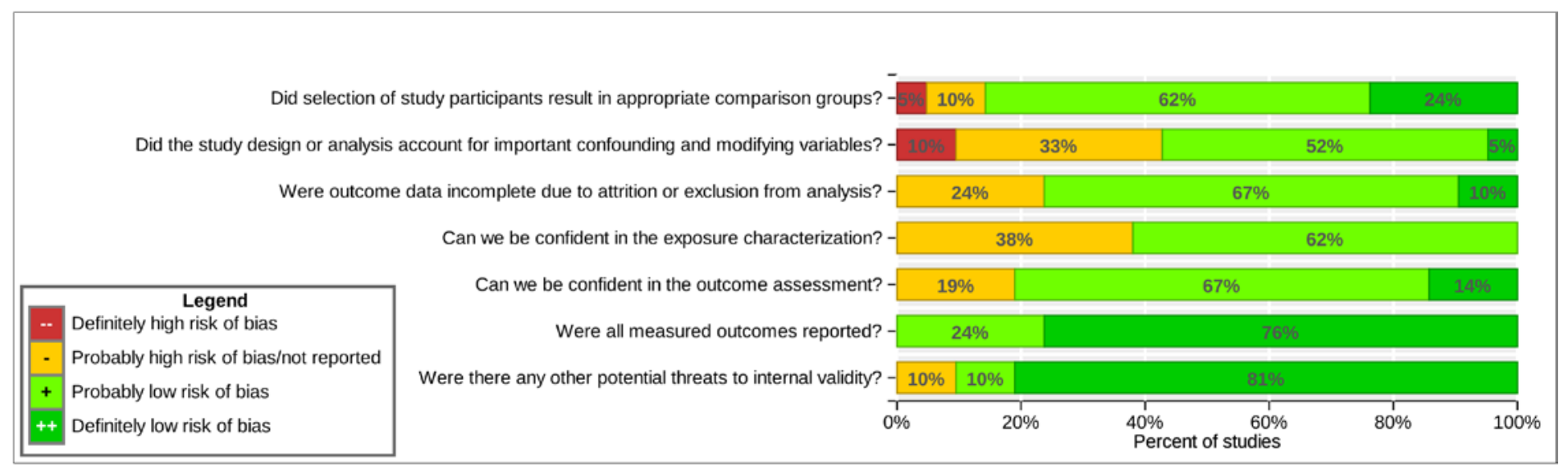

Figure E-9. Risk-of-bias Bar Graph for Studies Reporting on Reproductive and Developmental Toxicity, Other Than Spontaneous Abortion, Associated with Occupational Exposure to Cancer Chemotherapy

Interactive figure and additional study details in HAWC (NTP, 2015a).

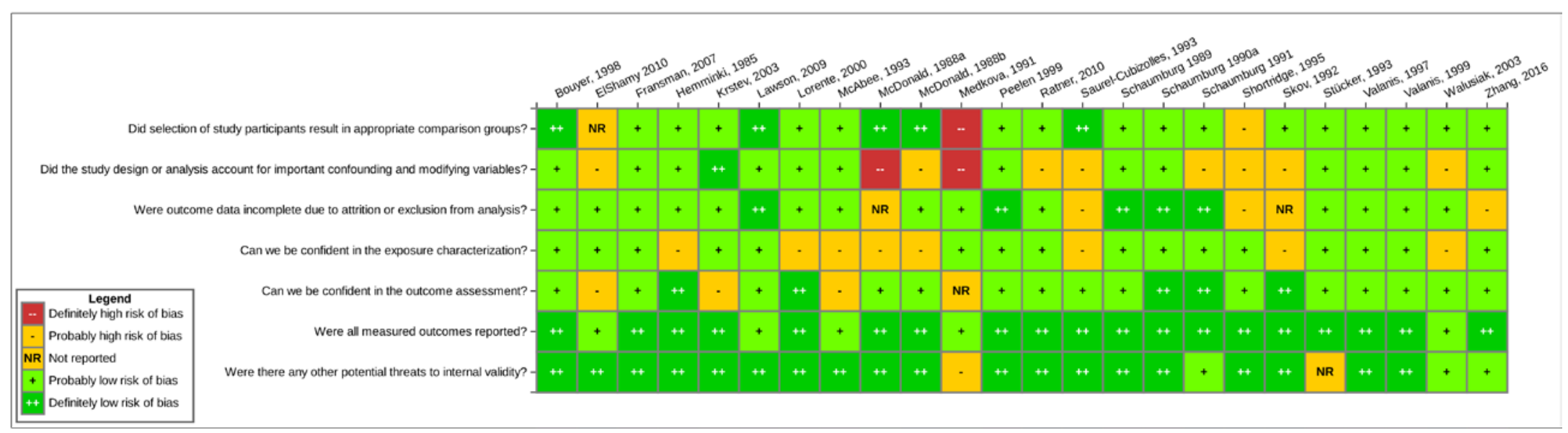

Figure E-10. Risk-of-bias Heat Map for Studies Reporting on Reproductive and Developmental Toxicity, Other Than Spontaneous Abortion, Associated with Occupational Exposure to Cancer Chemotherapy

Interactive figure and additional study details in HAWC (NTP, 2015a). Fransman 2007 refers to Fransman et al. (2007c). 


\section{Appendix F. Studies Reporting on Genetic Toxicity}

Table F-1. Studies Reporting on Genetic Toxicity, Including Chromosomal Aberrations, Micronucleus Induction, and DNA Damage Measured by Comet Assay

\begin{tabular}{|c|c|c|c|c|c|c|}
\hline Study & $\begin{array}{l}\text { Study Design } \\
\text { (Occupation, } \\
\text { Location, Sex) }\end{array}$ & No. of Participants & $\begin{array}{c}\text { Exposure Measure } \\
\text { (Duration) }\end{array}$ & Outcome Measured & Analysis & Results \\
\hline Anwar et al. (1994) & $\begin{array}{l}\text { Cross-sectional } \\
\text { (Nurses, National } \\
\text { Cancer Institute, Egypt, } \\
\text { F) }\end{array}$ & $\begin{array}{l}20 \text { exposed, } 20 \\
\text { unexposed nurses }\end{array}$ & $\begin{array}{l}\text { Job title and } \\
\text { interviewer-led } \\
\text { questionnaire } \\
\text { ( } 79.9 \pm 46.2 \text { months) } \\
\text { Note: Nurses routinely } \\
\text { operated under } \\
\text { inadequate protection }\end{array}$ & $\begin{array}{l}\text { \% cells with CA/ } 100 \\
\text { cells and no. of } \\
\text { MN/1,000 cells } \\
\text { (lymphocytes) }\end{array}$ & Chi-squared test & $\begin{array}{l}\text { Significantly higher \% } \\
\text { cells with CA and no. } \\
\text { of cells with MN } \\
\text { compared to control } \\
\text { nurses }\end{array}$ \\
\hline Bolognesi et al. (2005) & $\begin{array}{l}\text { Cross-sectional } \\
\text { (Nurses, IMPEA } \\
\text { project, Italy, FM) }\end{array}$ & $\begin{array}{l}\text { For lymphocytes: } \\
42 \text { exposed, } 43 \\
\text { unexposed university } \\
\text { professionals; for } \\
\text { buccal cells, } 50 \\
\text { exposed and } 50 \\
\text { unexposed }\end{array}$ & $\begin{array}{l}\text { Job title, [self-reported] } \\
\text { questionnaire, and } \\
\text { biomonitoring } \\
\text { (10.2 years) } \\
\text { Note: Adequate safety } \\
\text { equipment and PPE } \\
\text { were used }\end{array}$ & $\begin{array}{l}\text { No. of MN/1,000 cells } \\
\text { (lymphocytes or } \\
\text { buccal) }\end{array}$ & Mann-Whitney U-test & $\begin{array}{l}\text { No difference in no. of } \\
\text { MN (lymphocyte or } \\
\text { buccal) compared to } \\
\text { control subjects }\end{array}$ \\
\hline Boughattas et al. (2010) & $\begin{array}{l}\text { Cross-sectional } \\
\text { (Nurses, Tunisia, FM) } \\
\text { Note: Same population } \\
\text { as Bouraoui } 2011\end{array}$ & $\begin{array}{l}20 \text { exposed, } 20 \\
\text { unexposed } \\
\text { administrative staff }\end{array}$ & $\begin{array}{l}\text { Job title and self- } \\
\text { reported questionnaire } \\
\text { (range 1-16 years) } \\
\text { Note: } 80 \% \text { of nurses } \\
\text { used PPE; ward nurses } \\
\text { usually used a laminar } \\
\text { flow cabinet to prepare } \\
\text { doses, while outpatient } \\
\text { nurses usually prepared } \\
\text { drugs at patient's side } \\
\text { or in the hallway }\end{array}$ & $\begin{array}{l}\text { No. of cells with } \\
\text { MN/1,000 cells } \\
\text { (lymphocytes) }\end{array}$ & $\begin{array}{l}\text { Pearson' Chi-squared } \\
\text { test; ANOVA and } \\
\text { Student's t-test used to } \\
\text { test effect of age, sex, } \\
\text { exposure duration, } \\
\text { smoking, PPE, } \\
\text { cytostatic contact index } \\
\text { (CCI), family history of } \\
\text { cancer }\end{array}$ & $\begin{array}{l}\text { Significantly higher no. } \\
\text { of cells with MN in } \\
\text { exposed nurses; } \\
\text { significant effect of } \\
\text { CCI on outcome and } \\
\text { other variables were NS }\end{array}$ \\
\hline
\end{tabular}




\begin{tabular}{|c|c|c|c|c|c|c|}
\hline Study & $\begin{array}{l}\text { Study Design } \\
\text { (Occupation, } \\
\text { Location, Sex) }\end{array}$ & No. of Participants & $\begin{array}{c}\text { Exposure Measure } \\
\text { (Duration) }\end{array}$ & Outcome Measured & Analysis & Results \\
\hline Bouraoui et al. (2011) & $\begin{array}{l}\text { Cross-sectional } \\
\text { (Nurses, Tunisia, FM) } \\
\text { Note: Same population } \\
\text { as Boughattas } 2010\end{array}$ & $\begin{array}{l}20 \text { exposed, } 20 \\
\text { unexposed } \\
\text { administrative staff }\end{array}$ & $\begin{array}{l}\text { Job title and self- } \\
\text { reported questionnaire } \\
\text { (range }<5-10 \text { years) } \\
\text { Note: } 75 \% \text { of nurses } \\
\text { used gloves, } 10 \% \text { used } \\
\text { gowns, } 15 \% \text { used } \\
\text { masks, and } 5 \% \text { did not } \\
\text { use PPE }\end{array}$ & $\begin{array}{l}\text { No. of CA/100 cells } \\
\text { and no. of MN/1,000 } \\
\text { cells (lymphocytes) }\end{array}$ & $\begin{array}{l}\text { Student's-t-test; } \\
\text { multivariate regression } \\
\text { on effect of age, sex } \\
\text { exposure duration, } \\
\text { smoking, PPE, and } \\
\text { proliferation rate index } \\
\text { on CA }\end{array}$ & $\begin{array}{l}\text { Significantly higher no. } \\
\text { of CA and no. of MN } \\
\text { compared to unexposed } \\
\text { administrative staff; no } \\
\text { effect of variables on } \\
\text { CA }\end{array}$ \\
\hline $\begin{array}{l}\text { Buono-Michel et al. } \\
(2000)\end{array}$ & $\begin{array}{l}\text { Cross-sectional } \\
\text { (Nurses, France, FM) }\end{array}$ & $\begin{array}{l}14 \text { exposed, } 14 \\
\text { unexposed blood } \\
\text { donors }\end{array}$ & $\begin{array}{l}\begin{array}{l}\text { Job title and self- } \\
\text { reported questionnaire } \\
\text { (range } 1-28 \text { years) }\end{array} \\
\text { Note: No hood or } \\
\text { dedicated ventilation } \\
\text { available in drug } \\
\text { preparation area; most } \\
\text { nurses wore gloves and } \\
\text { accidental exposure } \\
\text { occurred to } 7 \text { nurses }\end{array}$ & $\begin{array}{l}\text { No. of cells with } \\
\text { MN/1,000 cells } \\
\text { (lymphocytes) }\end{array}$ & $\begin{array}{l}\text { Mann-Whitney U-test; } \\
\text { ANOVA tested effects } \\
\text { of age, family history of } \\
\text { cancer; correlation } \\
\text { analysis tested for } \\
\text { effect of smoking and } \\
\text { exposure duration }\end{array}$ & $\begin{array}{l}\text { Significantly higher no. } \\
\text { of cells with MN } \\
\text { compared to unexposed } \\
\text { blood donors; no } \\
\text { correlation or effect of } \\
\text { variables on MN }\end{array}$ \\
\hline Burgaz et al. (1999) & $\begin{array}{l}\text { Cross-sectional } \\
\text { (Nurses, Turkey, F) } \\
\text { Note: Overlapping } \\
\text { population with Burgaz } \\
2002\end{array}$ & $\begin{array}{l}\text { Lymphocytes: } 23 \\
\text { exposed, } 13 \text { unexposed } \\
\text { nurses and office staff; } \\
\text { for buccal cells, } 25 \text { and } \\
\text { 14, respectively }\end{array}$ & $\begin{array}{l}\text { Job title, interviewer- } \\
\text { led questionnaire, and } \\
\text { biomonitoring } \\
\text { ( } 5 \pm 4.45 \text { years) } \\
\text { Note: Nurses involved } \\
\text { in preparation and } \\
\text { administration of the } \\
\text { agents for at least } \\
1 \text { year, } 4-5 \text { times per } \\
\text { day, no safety hoods } \\
\text { were used, and } \\
\text { inconsistent PPE use }\end{array}$ & $\begin{array}{l}\text { No. of MN/1,000 cells } \\
\text { (lymphocytes or buccal } \\
\text { cells) }\end{array}$ & $\begin{array}{l}\text { Student's t-test or } \\
\text { Mann-Whitney U-test; } \\
\text { Pearson and Spearman } \\
\text { Rank test examined } \\
\text { influence of age, } \\
\text { smoking, exposure } \\
\text { duration, frequency of } \\
\text { handling }\end{array}$ & $\begin{array}{l}\text { Significantly higher no. } \\
\text { of MN in lymphocytes } \\
\text { and higher, but NS, no. } \\
\text { of } \mathbf{M N} \text { in buccal cells } \\
\text { compared to unexposed } \\
\text { subjects; no significant } \\
\text { effect of variables on } \\
\text { outcome }\end{array}$ \\
\hline
\end{tabular}




\begin{tabular}{|c|c|c|c|c|c|c|}
\hline Study & $\begin{array}{l}\text { Study Design } \\
\text { (Occupation, } \\
\text { Location, Sex) }\end{array}$ & No. of Participants & $\begin{array}{c}\text { Exposure Measure } \\
\text { (Duration) }\end{array}$ & Outcome Measured & Analysis & Results \\
\hline Burgaz et al. (2002) & $\begin{array}{l}\text { Cross-sectional } \\
\text { (Nurses, Turkey, F) } \\
\text { Note: Overlapping } \\
\text { population with Burgaz } \\
1999\end{array}$ & $\begin{array}{l}20 \text { exposed, } 18 \\
\text { unexposed secretaries } \\
\text { and nursing students }\end{array}$ & $\begin{array}{l}\text { Job title, [self-reported] } \\
\text { questionnaire, and } \\
\text { biomonitoring } \\
\text { ( } 8.15 \pm 4.70 \text { years) } \\
\text { Note: Nurses involved } \\
\text { in preparation and } \\
\text { administration of the } \\
\text { agents for at least } \\
1 \text { year with minimal } \\
\text { protection }\end{array}$ & $\begin{array}{l}\% \text { cells with CA/200 } \\
\text { cells (lymphocytes) }\end{array}$ & $\begin{array}{l}\text { Student's t-test or } \\
\text { Mann-Whitney U-test; } \\
\text { multivariate ANOVA } \\
\text { examining effect of } \\
\text { exposure, smoking, and } \\
\text { age; correlation } \\
\text { analysis on urinary CP, } \\
\text { frequency of handling, } \\
\text { and PPE }\end{array}$ & $\begin{array}{l}\text { Higher, but NS, \% cells } \\
\text { with CA compared to } \\
\text { unexposed subjects; } \\
\text { exposure a significant } \\
\text { factor in ANOVA; no } \\
\text { relationships observed } \\
\text { in correlation analysis }\end{array}$ \\
\hline Buschini et al. (2013) & $\begin{array}{l}\text { Cross-sectional } \\
\text { (Hospital nurses, Italy, } \\
\text { F) } \\
\text { Note: Same population } \\
\text { as Moretti } 2015\end{array}$ & $\begin{array}{l}63 \text { exposed, } 74 \\
\text { unexposed nurses }\end{array}$ & $\begin{array}{l}\text { Job title and [self- } \\
\text { reported] questionnaire } \\
\text { (110 } \pm 86 \text { months) }\end{array}$ & $\begin{array}{l}\text { \% Tail DNA } \\
\text { (leukocytes) }\end{array}$ & $\begin{array}{l}\text { Wilcoxson rank sum } \\
\text { test }\end{array}$ & $\begin{array}{l}\text { No difference in \% tail } \\
\text { DNA }\end{array}$ \\
\hline
\end{tabular}




\begin{tabular}{|c|c|c|c|c|c|c|}
\hline Study & $\begin{array}{l}\text { Study Design } \\
\text { (Occupation, } \\
\text { Location, Sex) }\end{array}$ & No. of Participants & $\begin{array}{c}\text { Exposure Measure } \\
\text { (Duration) }\end{array}$ & Outcome Measured & Analysis & Results \\
\hline Cavallo et al. (2005) & $\begin{array}{l}\text { Cross-sectional } \\
\text { (Ward and outpatient } \\
\text { nurses, and pharmacy } \\
\text { technicians, Italy, FM) } \\
\text { Note: Same population } \\
\text { as Ursini } 2006\end{array}$ & $\begin{array}{l}30 \text { exposed: } 13 \text { ward } \\
\text { nurses, } 12 \text { outpatient } \\
\text { nurses, } 5 \text { pharmacy } \\
\text { technicians, and } 30 \\
\text { unexposed } \\
\text { administrative staff }\end{array}$ & $\begin{array}{l}\text { Job title, [self-reported] } \\
\text { questionnaire, and } \\
\text { biomonitoring } \\
(6.2 \pm 2.9 \text { years, ward } \\
\text { nurses; } 7.0 \pm 2.0 \text { years, } \\
\text { pharmacists; } \\
8.1 \pm 6.0 \text { years, } \\
\text { outpatient nurses }) \\
\text { Note: All exposed } \\
\text { personnel used } \\
\text { adequate PPE, } \\
\text { including gloves, caps, } \\
\text { overalls, and goggles }\end{array}$ & $\begin{array}{l}\text { No. of CA/ } 100 \text { cells } \\
\text { (lymphocytes) and no. } \\
\text { of MN/1,000 cells } \\
\text { (lymphocytes or buccal } \\
\text { cells) }\end{array}$ & $\begin{array}{l}\text { ANOVA for CA, and Z } \\
\text { normal test for MN }\end{array}$ & $\begin{array}{l}\text { Significantly higher no. } \\
\text { of CA compared to } \\
\text { unexposed subjects; } \\
\text { significantly higher no. } \\
\text { of } \mathbf{M N} \text { in buccal cells } \\
\text { in ward and outpatient } \\
\text { nurses compared to } \\
\text { unexposed subjects; no. } \\
\text { of } \mathbf{M N} \text { in lymphocytes } \\
\text { NS }\end{array}$ \\
\hline Connor et al. (2010) & $\begin{array}{l}\text { Cross-sectional } \\
\text { (Nurses, pharmacists, } \\
\text { and pharmacy } \\
\text { technicians, USA, FM) } \\
\text { Note: Same population } \\
\text { as McDiarmid } 2010 \\
\text { and } 2014\end{array}$ & $\begin{array}{l}66 \text { exposed, } 52 \\
\text { unexposed nurses and } \\
\text { pharmacy personnel }\end{array}$ & $\begin{array}{l}\text { Job title, [self-reported] } \\
\text { questionnaire, 6-week } \\
\text { diary of drug-handling } \\
\text { events, biomonitoring, } \\
\text { and environmental } \\
\text { monitoring (>6 months) } \\
\text { Note: Exposed } \\
\text { personnel followed safe } \\
\text { handling practices as } \\
\text { per NIOSH, and used } \\
\text { biological safety } \\
\text { cabinets for drug } \\
\text { preparation }\end{array}$ & $\begin{array}{l}\% \text { Tail DNA and tail } \\
\text { moment }\end{array}$ & $\begin{array}{l}\text { Linear models adjusted } \\
\text { for covariates; } \\
\text { covariates included: } \\
\text { study site location, age, } \\
\text { glove usage, personal } \\
\text { medication use }\end{array}$ & $\begin{array}{l}\text { No difference in \% tail } \\
\text { DNA or tail moment; } \\
\text { covariate NS }\end{array}$ \\
\hline Cornetta et al. (2008) & $\begin{array}{l}\text { Cross-sectional } \\
\text { (Nurses, Italy, FM) } \\
\text { Note: Population } \\
\text { overlaps with Testa } \\
2007\end{array}$ & $\begin{array}{l}83 \text { exposed, } 73 \\
\text { unexposed } \\
\text { administrative staff }\end{array}$ & $\begin{array}{l}\text { Job title and } \\
\text { interviewer-led } \\
\text { questionnaire } \\
\text { (12.2 } \pm 7.3 \text { years) } \\
\text { Note: Exposed nurses } \\
\text { used adequate PPE and } \\
\text { vertical laminar flow } \\
\text { cabinet }\end{array}$ & $\begin{array}{l}\text { No. of MN/1,000 cells } \\
\text { (lymphocytes) and \% } \\
\text { tail DNA }\end{array}$ & $\begin{array}{l}\text { Mann-Whitney U-test } \\
\text { and unpaired t-test; } \\
\text { multivariate regression } \\
\text { examined influence of } \\
\text { age, sex, smoking, } \\
\text { exposure duration, and } \\
\text { genotype }\end{array}$ & $\begin{array}{l}\text { Significantly higher no. } \\
\text { of } \mathbf{M N} \text { and } \% \text { tail } \\
\text { DNA compared to } \\
\text { unexposed subjects; age } \\
\text { and sex were significant } \\
\text { variables for no. of } \mathbf{M N}\end{array}$ \\
\hline
\end{tabular}




\begin{tabular}{|c|c|c|c|c|c|c|}
\hline Study & $\begin{array}{l}\text { Study Design } \\
\text { (Occupation, } \\
\text { Location, Sex) }\end{array}$ & No. of Participants & $\begin{array}{c}\text { Exposure Measure } \\
\text { (Duration) }\end{array}$ & Outcome Measured & Analysis & Results \\
\hline Cooke et al. (1991) & $\begin{array}{l}\text { Cross-sectional } \\
\text { (Nurses, pharmacists } \\
\text { and pharmacy } \\
\text { technicians, United } \\
\text { Kingdom, sex NR) }\end{array}$ & $\begin{array}{l}61 \text { exposed, } 12 \\
\text { unexposed office } \\
\text { workers }\end{array}$ & $\begin{array}{l}\text { Job title and [self- } \\
\text { reported] questionnaire } \\
\text { (mc }>6 \text { months) } \\
\text { Note: Exposed } \\
\text { pharmacists used } \\
\text { biological safety } \\
\text { cabinets }\end{array}$ & $\begin{array}{l}\text { No. of CA } / 100 \text { cells } \\
\text { (lymphocytes) }\end{array}$ & $\begin{array}{l}\text { Chi-squared test for } \\
\text { individuals with } \leq 0.9 \\
\text { versus } \geq 1.0 \text { CA per } 100 \\
\text { cells; ANOVA of } \\
\text { transformed data }\end{array}$ & $\begin{array}{l}\text { No difference in no. of } \\
\text { CA compared to } \\
\text { unexposed controls }\end{array}$ \\
\hline Deng et al. (2005) & $\begin{array}{l}\text { Cross-sectional } \\
\text { (Methotrexate } \\
\text { production workers, } \\
\text { China, FM) }\end{array}$ & $\begin{array}{l}21 \text { exposed, } 21 \\
\text { unexposed [no } \\
\text { description] }\end{array}$ & $\begin{array}{l}\text { Job title, demographics, } \\
\text { and job tasks reported } \\
\text { [method NR] (range } 1- \\
14 \text { years) } \\
\text { Note: Inadequate } \\
\text { ventilation equipment; } \\
20 \text { of } 21 \text { workers used } \\
P P E\end{array}$ & $\begin{array}{l}\text { No. of cells with } \mathrm{MN} \\
\text { and no. of MN/1,000 } \\
\text { cells, tail length and tail } \\
\text { moment (lymphocytes) }\end{array}$ & $\begin{array}{l}\text { Student's t-test; } \\
\text { Kendall's test evaluated } \\
\text { correlation of exposure } \\
\text { duration }\end{array}$ & $\begin{array}{l}\text { Significantly higher no. } \\
\text { of cells with MN, no. } \\
\text { of } \mathbf{M N} \text {, and } \\
\text { significantly greater tail } \\
\text { length compared to } \\
\text { controls; significant } \\
\text { correlation with } \\
\text { exposure duration for } \\
\text { no. of cells with MN, } \\
\text { no. of } \mathbf{M N} \text {; tail } \\
\text { moment NS }\end{array}$ \\
\hline Deng et al. (2006) & $\begin{array}{l}\text { Cross-sectional } \\
\text { (Vincristine production } \\
\text { workers, China, FM) }\end{array}$ & $\begin{array}{l}15 \text { exposed, } 15 \\
\text { unexposed [no } \\
\text { description] }\end{array}$ & $\begin{array}{l}\text { Job title, work tasks and } \\
\text { safety equipment } \\
\text { availability [method } \\
\text { NR] (range } 1-20 \text { years) } \\
\text { Note: Inadequate } \\
\text { ventilation equipment; } \\
\text { all } 15 \text { workers used } \\
\text { PPE }\end{array}$ & $\begin{array}{l}\text { No. of cells with MN } \\
\text { and no. of MN/1,000 } \\
\text { cells (lymphocytes), tail } \\
\text { length and tail moment }\end{array}$ & $\begin{array}{l}\text { Student's t-test (tail } \\
\text { moment only), } \\
\text { Wilcoxon's rank sum; } \\
\text { Kendall's test evaluated } \\
\text { correlation of exposure } \\
\text { duration }\end{array}$ & $\begin{array}{l}\text { Significantly higher no. } \\
\text { of cells with MN, no. } \\
\text { of MN, and tail length; } \\
\text { tail moment NS }\end{array}$ \\
\hline El-Ebiary et al. (2013) & $\begin{array}{l}\text { Cross-sectional } \\
\text { (Nurses and } \\
\text { pharmacists, Egypt, F) }\end{array}$ & $\begin{array}{l}38 \text { exposed, } 30 \\
\text { unexposed nurses }\end{array}$ & $\begin{array}{l}\text { Job title and [self- } \\
\text { reported] questionnaire } \\
(8.18 \pm 4.55 \text { years }) \\
\text { Note: Nurse used } \\
\text { gloves, but not other } \\
\text { PPE; pharmacists used } \\
\text { adequate PPE and } \\
\text { worked with biological } \\
\text { safety cabinets }\end{array}$ & $\begin{array}{l}\text { \% cells with CA and } \\
\text { no. of CA/200 cells, no } \\
\text { of MN/1,000 cells } \\
\text { (lymphocytes) }\end{array}$ & $\begin{array}{l}\text { Student's t-test; } \\
\text { correlation analysis by } \\
\text { Pearson's test for age } \\
\text { and exposure duration }\end{array}$ & $\begin{array}{l}\text { Significantly higher \% } \\
\text { cells with CA, no. of } \\
\text { CA, and no. of MN } \\
\text { compared to unexposed } \\
\text { nurses; significant } \\
\text { Pearson's coefficient } \\
\text { for duration of exposure } \\
\text { for CA }\end{array}$ \\
\hline
\end{tabular}




\begin{tabular}{|c|c|c|c|c|c|c|}
\hline Study & $\begin{array}{l}\text { Study Design } \\
\text { (Occupation, } \\
\text { Location, Sex) }\end{array}$ & No. of Participants & $\begin{array}{c}\text { Exposure Measure } \\
\text { (Duration) }\end{array}$ & Outcome Measured & Analysis & Results \\
\hline Ensslin et al. (1997) & $\begin{array}{l}\text { Cross-sectional } \\
\text { (Pharmacy personnel, } \\
\text { Germany, FM) }\end{array}$ & $\begin{array}{l}13 \text { exposed, } 13 \\
\text { unexposed [no } \\
\text { description] }\end{array}$ & $\begin{array}{l}\text { Job title, work tasks and } \\
\text { drugs handled [method } \\
\text { NR], and biomonitoring } \\
\text { ( } 31.6 \pm 13.5 \text { months) } \\
\text { Note: Pharmacists used } \\
\text { vertical laminar flow } \\
\text { hood; gloves and gown } \\
\text { always worn }\end{array}$ & $\begin{array}{l}\text { No. of MN/1,000 cells } \\
\text { (lymphocytes) }\end{array}$ & Wilcoxon rank test & $\begin{array}{l}\text { No difference in no. of } \\
\text { MN compared to } \\
\text { unexposed subjects }\end{array}$ \\
\hline $\begin{array}{l}\text { Garaj-Vrhovac and } \\
\text { Kopjar (1998) }\end{array}$ & $\begin{array}{l}\text { Cross-sectional } \\
\text { (Nurses, Croatia, sex } \\
\text { NR) } \\
\text { Note: Same population } \\
\text { as Kasuba } 1999\end{array}$ & $\begin{array}{l}10 \text { exposed, } 10 \\
\text { unexposed [no } \\
\text { description] }\end{array}$ & $\begin{array}{l}\text { Job title only } \\
\text { (17.3 years) }\end{array}$ & $\begin{array}{l}\text { No. of MN/500 cells } \\
\text { (lymphocytes) }\end{array}$ & Chi-squared test & $\begin{array}{l}\text { Significantly higher no. } \\
\text { of } \mathbf{M N} \text { compared to } \\
\text { unexposed subjects }\end{array}$ \\
\hline Grummt et al. (1993) & $\begin{array}{l}\text { Cross-sectional } \\
\text { (Nurses and physicians, } \\
\text { Germany, FM) }\end{array}$ & $\begin{array}{l}106 \text { exposed, } 93 \\
\text { unexposed [no } \\
\text { description] }\end{array}$ & $\begin{array}{l}\text { Job title and self- } \\
\text { reported questionnaire } \\
\text { (range 1-39 years) } \\
\text { Note: Exposed } \\
\text { personnel prepared, } \\
\text { administered and } \\
\text { handled drugs with a } \\
\text { biological safety } \\
\text { cabinet }\end{array}$ & No. of CA/100 cells & $\begin{array}{l}\text { Mann-Whitney U-test, } \\
\text { including analyses of } \\
\text { data by smoking status, } \\
\text { age, and exposure } \\
\text { duration }\end{array}$ & $\begin{array}{l}\text { Significantly higher no. } \\
\text { of CA compared to } \\
\text { unexposed subjects }\end{array}$ \\
\hline Harris et al. (1992) & $\begin{array}{l}\text { Cross-sectional } \\
\text { (Nurses, USA, F) }\end{array}$ & $\begin{array}{l}24 \text { low exposure, } 21 \\
\text { moderate exposure, and } \\
19 \text { high exposure }\end{array}$ & $\begin{array}{l}\text { Job title and } \\
\text { interviewer-led } \\
\text { questionnaire (duration } \\
\text { NR) }\end{array}$ & $\begin{array}{l}\text { No. of MN/1,000 cells } \\
\text { (lymphocytes) }\end{array}$ & $\begin{array}{l}\text { Kruskal-Wallis one- } \\
\text { way ANOVA, } \\
\text { evaluated effect of age, } \\
\text { ionizing radiation, } \\
\text { chemicals or drugs, } \\
\text { exposure duration, } \\
\text { smoking, PPE }\end{array}$ & $\begin{array}{l}\text { No difference in no. of } \\
\text { MN compared to lower } \\
\text { exposed nurses; } \\
\text { significantly higher no. } \\
\text { of } \mathbf{M N} \text { was observed in } \\
\text { low and moderate } \\
\text { exposure nurses using } \\
\text { gloves }<100 \% \text { of the } \\
\text { time compared to } \\
\text { nurses using gloves } \\
100 \% \text { of the time }\end{array}$ \\
\hline
\end{tabular}




\begin{tabular}{|c|c|c|c|c|c|c|}
\hline Study & $\begin{array}{l}\text { Study Design } \\
\text { (Occupation, } \\
\text { Location, Sex) }\end{array}$ & No. of Participants & $\begin{array}{c}\text { Exposure Measure } \\
\text { (Duration) }\end{array}$ & Outcome Measured & Analysis & Results \\
\hline Hessel et al. (2001) & $\begin{array}{l}\text { Cross-sectional } \\
\text { (Nurses, pharmacy } \\
\text { personnel, one } \\
\text { physician, and one } \\
\text { orderly, Germany, FM) }\end{array}$ & $\begin{array}{l}113 \text { exposed (tested end } \\
\text { of } 1 \text { work week for } 3 \\
\text { cycles: } 83 \text { for cycle } 1 \text {, } \\
91 \text { for cycle } 2,73 \text { for } \\
\text { cycle } 3 \text { ), } 93 \text { baseline (3- } \\
\text { week absence from } \\
\text { exposure), and } 60 \\
\text { unexposed office } \\
\text { workers }\end{array}$ & $\begin{array}{l}\text { Job title, self-reported } \\
\text { questionnaire, and } \\
\text { biomonitoring } \\
\text { ( } 53 \text { months, range 1- } \\
144 \text { months) }\end{array}$ & $\begin{array}{l}\text { No. of MN/ } 1,000 \text { cells } \\
\text { (lymphocytes) - tested } \\
\text { beginning work shift } \\
\text { and } 3 \text { work cycles }\end{array}$ & $\begin{array}{l}\text { Mann-Whitney U and } \\
\text { Student's t-test; } \\
\text { Friedman's ANOVA to } \\
\text { test outcome over study } \\
\text { period; multiple linear } \\
\text { regression to test the } \\
\text { effect of age, ionizing } \\
\text { radiation, and exposure } \\
\text { status }\end{array}$ & $\begin{array}{l}\text { No difference in no. of } \\
\text { MN compared to } \\
\text { unexposed subjects on } \\
\text { cycle } 1 \text { or over the } \\
\text { entire study; ionizing } \\
\text { radiation exposure was } \\
\text { a significant factor in } \\
\text { MN at baseline; for } \\
\text { cycle } 1 \text { (highest } \\
\text { exposure cycle), age } \\
\text { was a significant factor, } \\
\text { but not ionizing } \\
\text { radiation or exposure } \\
\text { status }\end{array}$ \\
\hline Hola et al. (1988) & $\begin{array}{l}\text { Cross-sectional } \\
\text { (Nurses and physicians, } \\
\text { Czech Republic, FM) }\end{array}$ & $\begin{array}{l}136 \text { exposed (114 } \\
\text { nurses and } 22 \\
\text { physicians), } 20 \\
\text { unexposed blood } \\
\text { donors, } 277 \text { historical } \\
\text { unexposed controls }\end{array}$ & $\begin{array}{l}\text { Job title, number of } \\
\text { drug administrations, } \\
\text { and use of safety } \\
\text { cabinet [method NR] } \\
(9.3 \pm 0.7 \text { years, nurses } \\
\text { and } 10.3 \pm 1.4 \text { years, } \\
\text { physicians) }\end{array}$ & $\begin{array}{l}\text { \% cells with CA/100 } \\
\text { cells scored } \\
\text { (lymphocytes) }\end{array}$ & Student's t-test & $\begin{array}{l}\text { Significantly higher \% } \\
\text { cells with CA in } \\
\text { exposed nurses working } \\
\text { in pulmonary } \\
\text { department compared } \\
\text { to unexposed blood } \\
\text { donors; \% cells with } \\
\text { CA in exposed } \\
\text { physicians NS }\end{array}$ \\
\hline Izdes et al. (2009) & $\begin{array}{l}\text { Cross-sectional } \\
\text { (Nurses, Turkey, FM) }\end{array}$ & $\begin{array}{l}19 \text { exposed, } 19 \\
\text { unexposed office } \\
\text { workers }\end{array}$ & $\begin{array}{l}\text { Job title and self- } \\
\text { reported questionnaire } \\
(11.3 \pm 4.2 \text { years }) \\
\text { Note: Vertical flow } \\
\text { safety cabinet, gloves } \\
\text { and mask used during } \\
\text { preparation of drugs }\end{array}$ & $\begin{array}{l}\text { DNA damage index } \\
(0=\text { none, } 1=\text { low, } \\
2=\text { high migration })\end{array}$ & $\begin{array}{l}\text { Mann-Whitney U-test } \\
\text { with Bonferroni } \\
\text { correction; Pearson's or } \\
\text { Spearman's correlation } \\
\text { test for exposure } \\
\text { duration (years of } \\
\text { employment) }\end{array}$ & $\begin{array}{l}\text { Significantly greater } \\
\text { DNA damage index } \\
\text { compared to unexposed } \\
\text { office workers; no } \\
\text { correlation with years } \\
\text { of employment }\end{array}$ \\
\hline
\end{tabular}




\begin{tabular}{|c|c|c|c|c|c|c|}
\hline Study & $\begin{array}{l}\text { Study Design } \\
\text { (Occupation, } \\
\text { Location, Sex) }\end{array}$ & No. of Participants & $\begin{array}{c}\text { Exposure Measure } \\
\text { (Duration) }\end{array}$ & Outcome Measured & Analysis & Results \\
\hline Jakab et al. (2001) & $\begin{array}{l}\text { Cross-sectional } \\
\text { (Nurses, Hungary, F) }\end{array}$ & $\begin{array}{l}95 \text { exposed from } 4 \\
\text { hospitals, } 34 \text { unexposed } \\
\text { industrial workers, } 74 \\
\text { historical unexposed } \\
\text { controls }\end{array}$ & $\begin{array}{l}\text { Job title and } \\
\text { interviewer-led } \\
\text { questionnaire (mean } \\
\text { range } 4.0-6.5 \text { years } \\
\text { across } 4 \text { hospitals) } \\
\text { Note: Hospital } 1 \text { had no } \\
\text { safety equipment, } \\
\text { Hospital } 2 \text { had } \\
\text { insufficient safety } \\
\text { equipment, and } \\
\text { Hospital } 3 \text { and } 4 \text { had } \\
\text { sufficient equipment }\end{array}$ & $\begin{array}{l}\% \text { of cells with CA and } \\
\text { no. of CA/100 cells }\end{array}$ & $\begin{array}{l}\text { Student's t-test and } \\
\text { Mann-Whitney U-test }\end{array}$ & $\begin{array}{l}\text { Significantly higher \% } \\
\text { cells with CA and no. } \\
\text { of CA in exposed } \\
\text { nurses from all } 4 \\
\text { hospitals compared to } \\
\text { unexposed industrial } \\
\text { workers }\end{array}$ \\
\hline Kašuba et al. (1999) & $\begin{array}{l}\text { Cross-sectional } \\
\text { (Nurses, Croatia, F) } \\
\text { Note: Same population } \\
\text { as Garaj-Vrhovac and } \\
\text { Kopjar } 1998\end{array}$ & $\begin{array}{l}20 \text { exposed, } 16 \\
\text { unexposed [no } \\
\text { description] }\end{array}$ & $\begin{array}{l}\text { Job title, work tasks, } \\
\text { and drugs handled } \\
\text { [method NR] (range 1- } \\
31 \text { years) }\end{array}$ & $\begin{array}{l}\text { No. of cells with MN } \\
\text { and no. of MN/1,000 } \\
\text { cells (lymphocytes) }\end{array}$ & Chi-squared test & $\begin{array}{l}\text { Significantly higher no. } \\
\text { of cells with } \mathbf{M N} \text { and } \\
\text { no. of } \mathbf{M N} \text { in exposed } \\
\text { nurses, with significant } \\
\text { increases in nurses with } \\
\text { duration of exposure } \\
20-31 \text { years, but not } \\
11-14 \text { years compared } \\
\text { to compared to } \\
\text { unexposed subjects }\end{array}$ \\
\hline $\begin{array}{l}\text { Kevekordes et al. } \\
\text { (1998) }\end{array}$ & $\begin{array}{l}\text { Cross-sectional } \\
\text { (Nurses, Germany, FM) }\end{array}$ & $\begin{array}{l}10 \text { exposed ( } \mathrm{n}=10 \text { in } \\
\text { first examination } \\
2 \text { months after } \\
\text { installation of [vertical] } \\
\text { laminar flow safety } \\
\text { hood and } \mathrm{n}=6 \text { retested } \\
9 \text { months after } \\
\text { installation), } 10 \\
\text { unexposed [no } \\
\text { description] }\end{array}$ & $\begin{array}{l}\text { Job title and self- } \\
\text { reported questionnaire } \\
\text { (duration NR) } \\
\text { Note: Exposed nurses } \\
\text { reported following } \\
\text { recommended safety } \\
\text { precautions; PPE was } \\
\text { used }\end{array}$ & $\begin{array}{l}\text { No. of MN/1000 cells } \\
\text { (lymphocytes) }\end{array}$ & Mann-Whitney U-test & $\begin{array}{l}\text { Significantly higher no. } \\
\text { of MN compared to } \\
\text { unexposed subjects in } \\
\text { first examination } \\
\text { ( } 2 \text { months after } \\
\text { installation of safety } \\
\text { hood); second } \\
\text { examination ( } 9 \text { months } \\
\text { following installation of } \\
\text { safety hood) revealed } \\
\text { no difference in MN } \\
\text { compared to first } \\
\text { examination values for } \\
\text { exposed or unexposed } \\
\text { subjects }\end{array}$ \\
\hline
\end{tabular}




\begin{tabular}{|c|c|c|c|c|c|c|}
\hline Study & $\begin{array}{l}\text { Study Design } \\
\text { (Occupation, } \\
\text { Location, Sex) }\end{array}$ & No. of Participants & $\begin{array}{c}\text { Exposure Measure } \\
\text { (Duration) }\end{array}$ & Outcome Measured & Analysis & Results \\
\hline $\begin{array}{l}\text { Kopjar and Garaj- } \\
\text { Vrhovac (2001) }\end{array}$ & $\begin{array}{l}\text { Cross-sectional (Nurses } \\
\text { and physicians, Croatia, } \\
\text { FM) } \\
\text { Note: Overlapping } \\
\text { population with Kopjar } \\
2009\end{array}$ & $\begin{array}{l}50 \text { exposed, } 20 \\
\text { unexposed students and } \\
\text { office workers }\end{array}$ & $\begin{array}{l}\text { Job title and self- } \\
\text { reported questionnaire } \\
\text { (12.9 years, range } 1- \\
30 \text { years) } \\
\text { Note: PPE usage } \\
\text { differed among exposed } \\
\text { subjects; } n=20 \text { used } \\
\text { gloves only, } n=8 \text { used } \\
\text { gloves and masks, } \\
n=19 \text { used gloves and } \\
\text { safety cabinet with } \\
\text { vertical air flow, and } \\
n=3 \text { used gloves, } \\
\text { masks, and safety } \\
\text { cabinet with vertical air } \\
\text { flow }\end{array}$ & $\begin{array}{l}\text { \% Tail DNA, tail } \\
\text { length, and tail moment } \\
\text { (lymphocytes) }\end{array}$ & Mann-Whitney U-test & $\begin{array}{l}\text { Significantly greater \% } \\
\text { tail DNA, tail length } \\
\text { and tail moment } \\
\text { compared to unexposed } \\
\text { subjects; significantly } \\
\text { greater DNA damage } \\
\text { seen in exposed nurses } \\
\text { using only gloves } \\
\text { versus exposed nurses } \\
\text { using glove and safety } \\
\text { cabinet with vertical air } \\
\text { flow, or using glove, } \\
\text { mask, and safety } \\
\text { cabinet with vertical air } \\
\text { flow }\end{array}$ \\
\hline Kopjar et al. (2009) & $\begin{array}{l}\text { Cross-sectional } \\
\text { (Nurses and physicians, } \\
\text { Croatia, F) } \\
\text { Note: Overlapping } \\
\text { population with Kopjar } \\
\text { and Garaj-Vrhorac } \\
2001\end{array}$ & $\begin{array}{l}50 \text { exposed, } 50 \\
\text { unexposed students and } \\
\text { office workers }\end{array}$ & $\begin{array}{l}\text { Job title and self- } \\
\text { reported questionnaire } \\
\text { (12.9 years, range 1- } \\
30 \text { years) } \\
\text { Note: } 38 \% \text { of exposed } \\
\text { subjects used gloves } \\
\text { and vertical laminar } \\
\text { flow; } 16 \% \text { used gloves } \\
\text { and mask, while only } \\
6 \% \text { simultaneously used } \\
\text { gloves, mask, and } \\
\text { vertical laminar flow } \\
\text { cabinet }\end{array}$ & $\begin{array}{l}\text { \% cells with CA and } \\
\text { no. of CA/200 cells, no. } \\
\text { of cells with MN and } \\
\text { no. of MN/1,000 cells } \\
\text { and tail moment } \\
\text { (lymphocytes) }\end{array}$ & $\begin{array}{l}\text { Mann-Whitney U-test; } \\
\text { Spearman Rank Order } \\
\text { Correlations age, } \\
\text { smoking, exposure } \\
\text { duration, PPE; Kruskal- } \\
\text { Wallis ANOVA by } \\
\text { ranks }\end{array}$ & $\begin{array}{l}\text { Significantly higher \% } \\
\text { cells with CA, no. of } \\
\text { CA, no. of cells with } \\
\text { MN, no. of MN, and } \\
\text { tail length compared to } \\
\text { unexposed subjects; } \\
\text { highest level of tail } \\
\text { length observed in } \\
\text { exposed subjects who } \\
\text { only used latex gloves; } \\
\text { age and exposure } \\
\text { duration influenced } \\
\text { MN; longer exposure } \\
\text { duration was } \\
\text { accompanied with a } \\
\text { shift toward higher } \\
\text { values for tail length, \% } \\
\text { cells with CA and } \\
\text { number of cells with } \\
\text { MN }\end{array}$ \\
\hline
\end{tabular}




\begin{tabular}{|c|c|c|c|c|c|c|}
\hline Study & $\begin{array}{l}\text { Study Design } \\
\text { (Occupation, } \\
\text { Location, Sex) }\end{array}$ & No. of Participants & $\begin{array}{c}\text { Exposure Measure } \\
\text { (Duration) }\end{array}$ & Outcome Measured & Analysis & Results \\
\hline Krepinsky et al. (1990) & $\begin{array}{l}\text { Cross-sectional } \\
\text { (Nurses, Canada, F) }\end{array}$ & $\begin{array}{l}10 \text { exposed, } 10 \\
\text { unexposed nurses }\end{array}$ & $\begin{array}{l}\text { Job title, types and } \\
\text { frequency of drug } \\
\text { handling, and safety } \\
\text { equipment used } \\
\text { [method NR] (duration } \\
\text { NR) } \\
\text { Note: Exposed nurses } \\
\text { prepared drugs in } \\
\text { separate rooms without } \\
\text { safety hoods or special } \\
\text { ventilation; safety } \\
\text { education provided, but } \\
\text { PPE usage not } \\
\text { monitored }\end{array}$ & $\begin{array}{l}\text { No. of CA/300 cells } \\
\text { (lymphocytes) } \\
\text { measured at end of } \\
\text { work cycle and after 2- } \\
6 \text { days off work }\end{array}$ & Chi-squared test & $\begin{array}{l}\text { No difference in no. of } \\
\text { CA compared to } \\
\text { unexposed nurses at } \\
\text { end of work cycle or } \\
\text { after time off work; no. } \\
\text { of CA was higher, but } \\
\text { NS, compared to } \\
\text { unexposed nurses } \\
\text { (combining end of work } \\
\text { and after work samples) }\end{array}$ \\
\hline Ladeira et al. (2014) & $\begin{array}{l}\text { Cross-sectional } \\
\text { (Nurses, Portugal, FM) }\end{array}$ & $\begin{array}{l}27 \text { exposed, } 111 \\
\text { unexposed university } \\
\text { employees }\end{array}$ & $\begin{array}{l}\text { Job title, self-reported } \\
\text { questionnaire, and } \\
\text { environmental } \\
\text { monitoring ( } 6.01 \text { years, } \\
\text { range } 0.17-30 \text { years) }\end{array}$ & $\begin{array}{l}\text { No. of MN/1,000 cells } \\
\text { (lymphocytes) }\end{array}$ & $\begin{array}{l}\text { Mann-Whitney U-test, } \\
\text { multiple linear } \\
\text { regression of exposure, } \\
\text { age, sex, smoking and } \\
\text { alcohol consumption }\end{array}$ & $\begin{array}{l}\text { Significantly higher no. } \\
\text { of } \mathbf{M N} \text { compared to } \\
\text { unexposed subjects; } \\
\text { exposure and age were } \\
\text { significant variables }\end{array}$ \\
\hline Laffon et al. (2005) & $\begin{array}{l}\text { Cross-sectional Nurses, } \\
\text { Portugal, FM) }\end{array}$ & $\begin{array}{l}30 \text { exposed, } 22 \\
\text { unexposed nurses from } \\
\text { schools }\end{array}$ & $\begin{array}{l}\text { Job title and } \\
\text { interviewer-led } \\
\text { questionnaire } \\
\text { (6.40 } \pm 6.22 \text { years) } \\
\text { Note: Exposed nurses } \\
\text { used laminar airflow } \\
\text { hoods and wore } \\
\text { laboratory coat, mask, } \\
\text { and gloves while } \\
\text { preparing, mixing, and } \\
\text { administering these } \\
\text { drugs }\end{array}$ & $\begin{array}{l}\text { No. of MN/1,000 cells } \\
\text { (lymphocytes) and tail } \\
\text { length (leukocytes) }\end{array}$ & $\begin{array}{l}\text { ANOVA with Student's } \\
\text { t-test; overall F-test } \\
\text { assessed effect of } \\
\text { exposure duration, age, } \\
\text { sex, smoking and } \\
\text { genotypes }\end{array}$ & $\begin{array}{l}\text { Significantly greater } \\
\text { tail length compared to } \\
\text { unexposed subjects; } \\
\text { exposure duration was a } \\
\text { significant factor; no } \\
\text { difference in no. of } \mathbf{M N} \\
\text { compared to unexposed } \\
\text { subjects }\end{array}$ \\
\hline
\end{tabular}




\begin{tabular}{|c|c|c|c|c|c|c|}
\hline Study & $\begin{array}{l}\text { Study Design } \\
\text { (Occupation, } \\
\text { Location, Sex) }\end{array}$ & No. of Participants & $\begin{array}{c}\text { Exposure Measure } \\
\text { (Duration) }\end{array}$ & Outcome Measured & Analysis & Results \\
\hline $\begin{array}{l}\text { Machado-Santelli et al. } \\
\text { (1994) }\end{array}$ & $\begin{array}{l}\text { Cross-sectional } \\
\text { (Nurses, Brazil, FM) }\end{array}$ & $\begin{array}{l}25 \text { exposed, } 25 \\
\text { unexposed nurses, } \\
\text { physician, secretaries, } \\
\text { and drivers }\end{array}$ & $\begin{array}{l}\text { Job title and self- } \\
\text { reported questionnaire } \\
\text { (range 1-6 years) } \\
\text { Note: Only one nurse } \\
\text { used a safety hood, } 21 \\
\text { nurses used gloves, } 19 \\
\text { nurses used masks, and } \\
2 \text { nurses did not use } \\
P P E\end{array}$ & $\begin{array}{l}\text { No. of cells with } \\
\text { MN/1,000 cells (buccal } \\
\text { cells) }\end{array}$ & Chi-squared test & $\begin{array}{l}\text { Significantly higher no. } \\
\text { of cells with MN } \\
\text { compared to unexposed } \\
\text { subjects }\end{array}$ \\
\hline Mader et al. (2009) & $\begin{array}{l}\text { Cohort, longitudinal } \\
\text { (Nurses, Austria, F) } \\
\text { Note: Same population } \\
\text { as Pilger } 2000\end{array}$ & $\begin{array}{l}15 \text { subjects served as } \\
\text { their own controls: } \\
\text { exposed }(1,3,6 \text {, and } \\
9 \text { months on job, } \\
\text { referent, after a 3-week } \\
\text { vacation) }\end{array}$ & $\begin{array}{l}\text { Job title, self-reported } \\
\text { questionnaire, and } \\
\text { environmental } \\
\text { monitoring (range 1- } \\
17 \text { years) }\end{array}$ & $\begin{array}{l}\text { DNA damage index } \\
(0=<5 \%, 1=5-20 \% \text {, } \\
2=>20-40 \%, 3=>40- \\
95 \%, 4=>95 \%)\end{array}$ & $\begin{array}{l}\text { Kruskal-Wallis test } \\
\text { followed by a post-hoc } \\
\text { Dunn’s multiple } \\
\text { comparisons; trend } \\
\text { analysis by linear } \\
\text { regression }\end{array}$ & $\begin{array}{l}\text { Significantly higher } \\
\text { DNA damage } \\
\text { compared to referent } \\
\text { using trend analysis } \\
(\mathrm{p}=0.02) \text {; no } \\
\text { correlation with years } \\
\text { of exposure }\end{array}$ \\
\hline Mahmoodi et al. (2017) & $\begin{array}{l}\text { Cross-sectional (Nurses } \\
\text { and pharmacy } \\
\text { technicians, Iran, FM) }\end{array}$ & $\begin{array}{l}71 \text { exposed nurses, } 10 \\
\text { exposed pharmacy } \\
\text { technicians, } 74 \\
\text { unexposed nurses }\end{array}$ & $\begin{array}{l}\text { Job title and self- } \\
\text { reported questionnaire } \\
\text { (duration }>6 \text { months } \\
\text { and worked the week } \\
\text { prior to assessment) }\end{array}$ & $\begin{array}{l}\text { No. of CA/100 cells } \\
\text { and no. of MN/1,000 } \\
\text { cells (lymphocytes) }\end{array}$ & Student's t-test & $\begin{array}{l}\text { Significantly higher no. } \\
\text { of CA and no. of } \mathbf{M N} \\
\text { compared to unexposed } \\
\text { nurses }\end{array}$ \\
\hline
\end{tabular}




\begin{tabular}{|c|c|c|c|c|c|c|}
\hline Study & $\begin{array}{l}\text { Study Design } \\
\text { (Occupation, } \\
\text { Location, Sex) }\end{array}$ & No. of Participants & $\begin{array}{c}\text { Exposure Measure } \\
\text { (Duration) }\end{array}$ & Outcome Measured & Analysis & Results \\
\hline $\begin{array}{l}\text { Maluf and Erdtmann } \\
\text { (2000b) }\end{array}$ & $\begin{array}{l}\text { Cross-sectional (Nurses } \\
\text { and pharmacists, Brazil, } \\
\text { FM) }\end{array}$ & $\begin{array}{l}\text { First study: } 10 \text { exposed } \\
\text { and } 10 \text { unexposed } \\
\text { workers; follow-up } \\
\text { study: } 12 \text { exposed and } \\
12 \text { unexposed; } 34 \\
\text { historical unexposed } \\
\text { controls }\end{array}$ & $\begin{array}{l}\text { Job title and self- } \\
\text { reported questionnaire } \\
\text { (range } 1-6 \text { years) }\end{array}$ & $\begin{array}{l}\text { No. of MN/2,000 cells } \\
\text { and DNA damage index } \\
(0=<5 \%, 1=5-20 \% \text {, } \\
2=>20-40 \%, 3=>40- \\
95 \%, 4=>95 \%) \\
\text { (lymphocytes) }\end{array}$ & $\begin{array}{l}\text { Mann-Whitney U-test; } \\
\text { Spearman rank test for } \\
\text { correlations for age and } \\
\text { smoking }\end{array}$ & $\begin{array}{l}\text { In first study, } \\
\text { significantly higher no. } \\
\text { of MN compared to } \\
\text { concurrent unexposed } \\
\text { workers; in follow-up } \\
\text { study, no difference in } \\
\text { no. of MN and } \\
\text { significantly higher } \\
\text { DNA damage index } \\
\text { (only measured in } \\
\text { follow-up study) } \\
\text { compared to both } \\
\text { unexposed groups; no } \\
\text { correlation with age or } \\
\text { smoking }\end{array}$ \\
\hline $\begin{array}{l}\text { McDiarmid et al. } \\
\text { (2010) }\end{array}$ & $\begin{array}{l}\text { Cross-sectional } \\
\text { (Nurses, pharmacists, } \\
\text { and pharmacy } \\
\text { technicians, USA, FM) } \\
\text { Note: Same population } \\
\text { as Connor } 2010 \text { and } \\
\text { McDiarmid } 2014\end{array}$ & $\begin{array}{l}63 \text { exposed, } 46 \\
\text { unexposed nursing and } \\
\text { pharmacy personnel }\end{array}$ & $\begin{array}{l}\text { Job title, interviewer- } \\
\text { led questionnaire, and } \\
\text { 6-week diary of drug- } \\
\text { handling events } \\
\text { (duration }>6 \text { months } \\
\text { and worked } 24 \text { hours in } \\
\text { week prior to } \\
\text { assessment) } \\
\text { Note: Exposed } \\
\text { personnel followed safe } \\
\text { handling practices, } \\
\text { including drug } \\
\text { preparation in a } \\
\text { biological safety } \\
\text { cabinet, use of gloves, } \\
\text { work practices to } \\
\text { reduce drug } \\
\text { aerosolization }\end{array}$ & $\begin{array}{l}\text { No. of CA/200 cells } \\
\text { scored and IRR of CA } \\
\text { on chromosomes 5, 7, } \\
\text { and } 11 \text { (lymphocytes) }\end{array}$ & $\begin{array}{l}\text { Poisson regression } \\
\text { (categorical analysis) } \\
\text { and Poisson distribution } \\
\text { and log link function } \\
\text { (continuous variable for } \\
\text { frequency of drug } \\
\text { handling) }\end{array}$ & $\begin{array}{l}\text { Significantly higher no. } \\
\text { of CA and IRR of CA } \\
\text { on chromosome } 5 \text { of } \\
\text { highest exposed nurses } \\
\text { compared to unexposed } \\
\text { subjects; significantly } \\
\text { higher IRR of CA on } \\
\text { chromosome } 5 \text { with } \\
\text { increasing handling } \\
\text { events of all cancer } \\
\text { chemotherapy or } \\
\text { alkylating agents only; } \\
\text { IRR of CA on } \\
\text { chromosome } 7 \text { or } 11 \\
\text { NS }\end{array}$ \\
\hline
\end{tabular}




\begin{tabular}{|c|c|c|c|c|c|c|}
\hline Study & $\begin{array}{l}\text { Study Design } \\
\text { (Occupation, } \\
\text { Location, Sex) }\end{array}$ & No. of Participants & $\begin{array}{c}\text { Exposure Measure } \\
\text { (Duration) }\end{array}$ & Outcome Measured & Analysis & Results \\
\hline $\begin{array}{l}\text { McDiarmid et al. } \\
\text { (2014) }\end{array}$ & $\begin{array}{l}\text { Cross-sectional } \\
\text { (Nurses, pharmacists, } \\
\text { and pharmacy } \\
\text { technicians, USA, FM) } \\
\text { Note: Same population } \\
\text { as Connor } 2010 \text { and } \\
\text { McDiarmid } 2010\end{array}$ & $\begin{array}{l}63 \text { exposed, } 46 \\
\text { unexposed nursing and } \\
\text { pharmacy personnel }\end{array}$ & $\begin{array}{l}\text { Job title, interviewer- } \\
\text { led questionnaire, and } \\
6 \text {-week diary of drug- } \\
\text { handling events } \\
\text { (duration }>6 \text { months } \\
\text { and worked } 24 \text { hours in } \\
\text { week prior to } \\
\text { assessment) } \\
\text { Note: Exposed } \\
\text { personnel followed safe } \\
\text { handling practices, } \\
\text { including drug } \\
\text { preparation in a } \\
\text { biological safety } \\
\text { cabinet, use of gloves, } \\
\text { work practices to } \\
\text { reduce drug } \\
\text { aerosolization }\end{array}$ & $\begin{array}{l}\text { IRR of CA on } \\
\text { chromosomes 5, 7, and } \\
11 \text { for non-alkylating } \\
\text { agent exposure/200 } \\
\text { cells scored } \\
\text { (lymphocytes) }\end{array}$ & $\begin{array}{l}\text { Poisson regression } \\
\text { (categorical analysis) } \\
\text { and Poisson distribution } \\
\text { and log link function } \\
\text { (continuous variable for } \\
\text { frequency of drug } \\
\text { handling) }\end{array}$ & $\begin{array}{l}\text { Significantly higher } \\
\text { IRR of CA on } \\
\text { chromosome } 5 \text { with } \\
\text { increasing handling } \\
\text { events of non- } \\
\text { alkylating cancer } \\
\text { chemotherapy agents; } \\
\text { IRR of CA on } \\
\text { chromosome } 7 \text { or } 11 \\
\text { NS }\end{array}$ \\
\hline Medková (1990) & $\begin{array}{l}\text { Cross-sectional } \\
\text { (Physicians, nurses, } \\
\text { auxiliary workers, } \\
\text { Czech Republic, FM) }\end{array}$ & $\begin{array}{l}40 \text { exposed from } \\
\text { Department of } \\
\text { Radiotherapy, } 51 \\
\text { unexposed ( } 20 \text { medical } \\
\text { studies and } 31 \text { workers } \\
\text { from Department of } \\
\text { Anesthesiology and } \\
\text { Resuscitation) }\end{array}$ & $\begin{array}{l}\text { Job title and [self- } \\
\text { reported] questionnaire } \\
\text { (range } 0-15 \text { years) }\end{array}$ & $\begin{array}{l}\text { \% cells with CA/100 } \\
\text { cells scored } \\
\text { (lymphocytes) }\end{array}$ & Student's t-test & $\begin{array}{l}\text { Significantly higher \% } \\
\text { of cells with CA } \\
\text { compared to unexposed } \\
\text { subjects }\end{array}$ \\
\hline $\begin{array}{l}\text { Milković-Kraus and } \\
\text { Horvat (1991) }\end{array}$ & $\begin{array}{l}\text { Cross-sectional } \\
\text { (Nurses, Yugoslavia, F) }\end{array}$ & $\begin{array}{l}42 \text { exposed, } 42 \\
\text { unexposed nurses }\end{array}$ & $\begin{array}{l}\text { Job title and [self- } \\
\text { reported] questionnaire } \\
\text { (duration NR) } \\
\text { Note: None of nurses } \\
\text { routinely used PPE }\end{array}$ & $\begin{array}{l}\text { No. of CA/200 cells } \\
\text { (lymphocytes) }\end{array}$ & $\begin{array}{l}\text { Sign test for paired } \\
\text { samples; linear } \\
\text { regression analysis for } \\
\text { possible age-dependent } \\
\text { increase of observed } \\
\text { variables }\end{array}$ & $\begin{array}{l}\text { Significantly higher no. } \\
\text { of CA compared to } \\
\text { unexposed nurses; age } \\
\text { was not a significant } \\
\text { factor }\end{array}$ \\
\hline
\end{tabular}




\begin{tabular}{|c|c|c|c|c|c|c|}
\hline Study & $\begin{array}{l}\text { Study Design } \\
\text { (Occupation, } \\
\text { Location, Sex) }\end{array}$ & No. of Participants & $\begin{array}{c}\text { Exposure Measure } \\
\text { (Duration) }\end{array}$ & Outcome Measured & Analysis & Results \\
\hline Moretti et al. (2013) & $\begin{array}{l}\text { Cross-sectional } \\
\text { (Nurses, pharmacists, } \\
\text { and support personnel, } \\
\text { Italy, FM) } \\
\text { Note: Same population } \\
\text { as Villarini } 2011\end{array}$ & $\begin{array}{l}52 \text { exposed, } 52 \\
\text { unexposed blood } \\
\text { donors }\end{array}$ & $\begin{array}{l}\text { Job position and [self- } \\
\text { reported] questionnaire } \\
\text { (<10 years, } 65 \% \text {; } \\
>10 \text { years, } 35 \%) \\
\text { Note: } 3 \text { nurses used } \\
\text { gloves only, } 41 \text { used } \\
\text { gloves and mask, and } 8 \\
\text { used no protection }\end{array}$ & $\begin{array}{l}\text { No. of MN/1,000 cells } \\
\text { and \% tail DNA } \\
\text { (lymphocytes) }\end{array}$ & $\begin{array}{l}\text { Mann-Whitney U-test } \\
\text { followed by Whitney } \\
\text { U-test and Bonferroni } \\
\text { correction; multiple } \\
\text { linear regression tested } \\
\text { effects of exposure, sex, } \\
\text { age, smoking, type of } \\
\text { occupation, exposure } \\
\text { duration, and PPE }\end{array}$ & $\begin{array}{l}\text { Significantly higher \% } \\
\text { tail DNA compared to } \\
\text { unexposed blood } \\
\text { donors; > } 10 \text { years of } \\
\text { employer had } \\
\text { significantly higher } \\
\text { levels of \% tail DNA } \\
\text { than less years } \\
\text { employed; no. of MN } \\
\text { NS }\end{array}$ \\
\hline Moretti et al. (2015) & $\begin{array}{l}\text { Cross-sectional } \\
\text { (Nurses, Italy, F) } \\
\text { Note: Same population } \\
\text { as Buschini } 2013\end{array}$ & $\begin{array}{l}71 \text { exposed, } 77 \\
\text { unexposed nurses (70 } \\
\text { exposed and } 75 \\
\text { unexposed for no. of } \\
\text { CA/100 cells) }\end{array}$ & $\begin{array}{l}\text { Job title, self-reported } \\
\text { questionnaire, } \\
\text { biomonitoring, and } \\
\text { environmental } \\
\text { monitoring } \\
\text { (9.20 } \pm 7.18 \text { years) }\end{array}$ & $\begin{array}{l}\text { No. of CA/100 cells } \\
\text { and no. of MN/1,000 } \\
\text { cells } \\
\text { (lymphocytes) }\end{array}$ & $\begin{array}{l}\text { Mann-Whitney U-test; } \\
\text { followed by Mann- } \\
\text { Whitney U-test post- } \\
\text { hoc analysis with } \\
\text { Bonferroni correction; } \\
\text { multiple linear } \\
\text { regression tested effects } \\
\text { of exposure, age, type } \\
\text { of occupation, exposure } \\
\text { duration, PPE and } \\
\text { urinary CP }\end{array}$ & $\begin{array}{l}\text { Significantly higher no. } \\
\text { of CA and no. of } \mathbf{M N} \\
\text { compared to unexposed } \\
\text { nurses; variance was } \\
\text { mainly explained by the } \\
\text { subjects' type of } \\
\text { occupation (another } \\
\text { measure of exposure) }\end{array}$ \\
\hline Mrđanović et al. (2012) & $\begin{array}{l}\text { Cross-sectional } \\
\text { (Nurses, Bosnia and } \\
\text { Herzegovina, F) }\end{array}$ & $\begin{array}{l}15 \text { exposed, } 20 \\
\text { unexposed workers [no } \\
\text { description provided] }\end{array}$ & $\begin{array}{l}\text { Job title and self- } \\
\text { reported questionnaire } \\
\text { (range } 1-20 \text { years) } \\
\text { Note: Nurses used } \\
\text { gloves, masks, and } \\
\text { protective clothes }\end{array}$ & $\begin{array}{l}\text { No. of MN/1,000 cells } \\
\text { (lymphocytes) }\end{array}$ & $\begin{array}{l}\text { Mann-Whitney U-test } \\
\text { and ANOVA }\end{array}$ & $\begin{array}{l}\text { Significantly higher no. } \\
\text { of } \mathbf{M N} \text { in exposed } \\
\text { nurses }\end{array}$ \\
\hline Mušák et al. (2006) & $\begin{array}{l}\text { Cross-sectional } \\
\text { (Healthcare workers, } \\
\text { Slovakia, FM) } \\
\text { Note: Same population } \\
\text { as Mušák } 2013\end{array}$ & $\begin{array}{l}72 \text { exposed, } 34 \\
\text { unexposed hospital } \\
\text { workers }\end{array}$ & $\begin{array}{l}\text { Job title } \\
(8.09 \pm 7.30 \text { years })\end{array}$ & $\begin{array}{l}\text { No. of CA/100 cells } \\
\text { (lymphocytes) }\end{array}$ & Mann-Whitney U-test & $\begin{array}{l}\text { Higher, but NS } \\
\text { ( } \mathrm{p}=0.07) \text {, no. of CA } \\
\text { compared to unexposed } \\
\text { workers }\end{array}$ \\
\hline Mušák et al. (2013) & $\begin{array}{l}\text { Cross-sectional (Nurses } \\
\text { and physicians, } \\
\text { Slovakia, FM) } \\
\text { Note: Same population } \\
\text { as Mušák } 2006\end{array}$ & $\begin{array}{l}249 \text { exposed, } 250 \\
\text { unexposed hospital } \\
\text { workers }\end{array}$ & $\begin{array}{l}\text { Job title and self- } \\
\text { reported questionnaire } \\
(8.5 \pm 8.6 \text { years })\end{array}$ & $\begin{array}{l}\text { \% cells with }>1 \text { CA } \\
\text { (lymphocytes) }\end{array}$ & $\begin{array}{l}\text { Mann-Whitney U-test } \\
\text { and Kruskal-Wallis test } \\
\text { and logistic regression } \\
\text { tested effect of } \\
\text { exposure, age, sex, and } \\
\text { smoking }\end{array}$ & $\begin{array}{l}\text { Significantly higher \% } \\
\text { cells with >1 CA } \\
\text { compared to unexposed } \\
\text { workers; exposure was } \\
\text { only significant } \\
\text { variable }\end{array}$ \\
\hline
\end{tabular}




\begin{tabular}{|c|c|c|c|c|c|c|}
\hline Study & $\begin{array}{l}\text { Study Design } \\
\text { (Occupation, } \\
\text { Location, Sex) }\end{array}$ & No. of Participants & $\begin{array}{c}\text { Exposure Measure } \\
\text { (Duration) }\end{array}$ & Outcome Measured & Analysis & Results \\
\hline Nikula et al. (1984) & $\begin{array}{l}\text { Cross-sectional } \\
\text { (Nurses, Finland, [F]) }\end{array}$ & $\begin{array}{l}11 \text { exposed, } 16 \\
\text { unexposed (11 hospital } \\
\text { clerks and } 5 \text { laboratory } \\
\text { workers) }\end{array}$ & $\begin{array}{l}\text { Job title and } \\
\text { interviewer-led } \\
\text { questionnaire (range } \\
\text { 2.5-10 years) } \\
\text { Note: After 1980, } \\
\text { improvements were } \\
\text { made to work practices } \\
\text { and safety equipment } \\
\text { (e.g., biological safety } \\
\text { cabinet and isolated } \\
\text { room for drug } \\
\text { handling); nurses wear } \\
\text { gloves and masks }\end{array}$ & $\begin{array}{l}\text { \% cells with CA } \\
\text { (lymphocytes) }\end{array}$ & Mann-Whitney U-test & $\begin{array}{l}\text { Significantly higher \% } \\
\text { cells with CA } \\
\text { compared to either } \\
\text { unexposed hospital } \\
\text { clerks or unexposed } \\
\text { laboratory workers }\end{array}$ \\
\hline Pilger et al. (2000) & $\begin{array}{l}\text { Cross-sectional } \\
\text { (Pharmacy personnel, } \\
\text { Austria, FM) } \\
\text { Note: Same populations } \\
\text { as Mader } 2009\end{array}$ & $\begin{array}{l}16 \text { exposed, } 16 \\
\text { unexposed workers } \\
\text { from same hospital who } \\
\text { were tested at } 5 \text { time } \\
\text { points }\end{array}$ & $\begin{array}{l}\text { Job title, self-reported } \\
\text { questionnaire, and } \\
\text { biomonitoring (duration } \\
\text { NR) } \\
\text { Note: Hospitals had } \\
\text { central workplaces } \\
\text { containing cabinets } \\
\text { with vertical laminar } \\
\text { flow and personnel } \\
\text { wear glove, overalls, } \\
\text { caps, and goggles }\end{array}$ & $\begin{array}{l}\text { No. of MN/2,000 cells } \\
\text { (lymphocytes) }\end{array}$ & $\begin{array}{l}\text { ANOVA with } \\
\text { stochastic components } \\
\text { to compare data on the } \\
\text { same subjects over } 5 \\
\text { sampling periods }\end{array}$ & $\begin{array}{l}\text { No difference in the no. } \\
\text { of } \mathbf{M N} \text { compared to } \\
\text { unexposed subjects }\end{array}$ \\
\hline Pohlová et al. (1986) & $\begin{array}{l}\text { Cross-sectional } \\
\text { (Chemists, laboratory } \\
\text { assistants, pilot plant } \\
\text { workers, Czech } \\
\text { Republic, FM) }\end{array}$ & $\begin{array}{l}38 \text { exposed ( } 8 \text { pilot } \\
\text { workers and } 30 \\
\text { chemists and laboratory } \\
\text { assistants), } 18 \\
\text { unexposed librarians } \\
\text { and clerks }\end{array}$ & $\begin{array}{l}\text { Job title and self- } \\
\text { administered } \\
\text { questionnaire } \\
\text { (range }>1-<6 \text { years) }\end{array}$ & $\begin{array}{l}\text { \% cells with CA } \\
\text { (lymphocytes) }\end{array}$ & Student's t-test & $\begin{array}{l}\text { Significantly higher \% } \\
\text { of cells with CA } \\
\text { compared to unexposed } \\
\text { workers }\end{array}$ \\
\hline
\end{tabular}




\begin{tabular}{|c|c|c|c|c|c|c|}
\hline Study & $\begin{array}{l}\text { Study Design } \\
\text { (Occupation, } \\
\text { Location, Sex) }\end{array}$ & No. of Participants & $\begin{array}{c}\text { Exposure Measure } \\
\text { (Duration) }\end{array}$ & Outcome Measured & Analysis & Results \\
\hline Rekhadevi et al. (2007) & $\begin{array}{l}\text { Cross-sectional } \\
\text { (Nurses, India, F) }\end{array}$ & $\begin{array}{l}60 \text { exposed, } 60 \\
\text { unexposed from general } \\
\text { population }\end{array}$ & $\begin{array}{l}\text { Job title, interviewer- } \\
\text { led questionnaire, and } \\
\text { biomonitoring (range } \\
6 \text {-23 years) }\end{array}$ & $\begin{array}{l}\text { No. of MN/1,000 cells } \\
\text { (lymphocytes or buccal } \\
\text { cells), and tail length } \\
\text { (lymphocytes) }\end{array}$ & $\begin{array}{l}\text { Student's t-test; } \\
\text { multiple linear } \\
\text { regression for age, } \\
\text { exposure duration, daily } \\
\text { exposure }\end{array}$ & $\begin{array}{l}\text { Significantly higher no. } \\
\text { of MN (buccal or } \\
\text { lymphocytes) and tail } \\
\text { length compared to } \\
\text { unexposed subjects; age } \\
\text { and exposure duration } \\
\text { were significant for MN } \\
\text { and tail length; } \\
\text { significantly higher tail } \\
\text { length for nurses } \\
\text { with } \geq 10 \text { years } \\
\text { exposure compared } \\
\text { to }<10 \text { years exposure }\end{array}$ \\
\hline $\begin{array}{l}\text { Rodríguez-Montero et } \\
\text { al. (2016) }\end{array}$ & $\begin{array}{l}\text { Cross-sectional } \\
\text { (Medical Oncology } \\
\text { Service workers, Cuba, } \\
\text { FM) }\end{array}$ & $\begin{array}{l}14 \text { exposed: } 10 \text { workers } \\
\text { preparing doses in the } \\
\text { Central Unit of } \\
\text { Cytostatic Mixes } \\
\text { (CUCM) and } 4 \text { workers } \\
\text { of the Ambulatory } \\
\text { Chemotherapy Room } \\
\text { (ACR), } 14 \text { unexposed } \\
\text { workers at same } \\
\text { medical institution }\end{array}$ & $\begin{array}{l}\text { Job title, job tasks, } \\
\text { duration, and frequency } \\
\text { of handling drugs } \\
\text { [method NR] (mean } \\
\text { range } 12.8- \\
129.6 \text { months) } \\
\text { Note: Drugs were } \\
\text { prepared in CUCM } \\
\text { with correct use of } \\
\text { primary protective } \\
\text { barriers; good } \\
\text { laboratory practice is } \\
\text { employed in both the } \\
\text { CUCM and the ACR }\end{array}$ & $\begin{array}{l}\text { No. of cells with } \\
\text { MN/ } 1,000 \text { cell (buccal } \\
\text { cells) }\end{array}$ & Mann-Whitney U-test & $\begin{array}{l}\text { No difference in no. of } \\
\text { MN compared to } \\
\text { unexposed subjects }\end{array}$ \\
\hline
\end{tabular}




\begin{tabular}{|c|c|c|c|c|c|c|}
\hline Study & $\begin{array}{l}\text { Study Design } \\
\text { (Occupation, } \\
\text { Location, Sex) }\end{array}$ & No. of Participants & $\begin{array}{c}\text { Exposure Measure } \\
\text { (Duration) }\end{array}$ & Outcome Measured & Analysis & Results \\
\hline Rombaldi et al. (2009) & $\begin{array}{l}\text { Cross-sectional (Nurses } \\
\text { and pharmacists, Brazil, } \\
\text { FM) }\end{array}$ & $\begin{array}{l}20 \text { exposed, } 20 \\
\text { unexposed office } \\
\text { workers }\end{array}$ & 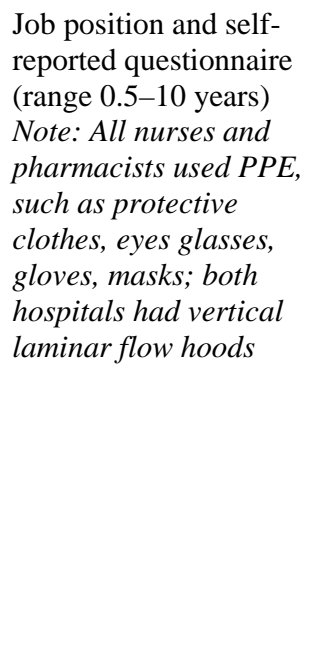 & $\begin{array}{l}\text { No. of cells with } \\
\text { MN/1,000 cells and } \\
\text { DNA damage index } \\
(0=<5 \%, 1=5-20 \% \text {, } \\
2=>20-40 \%, 3=>40- \\
95 \%, 4=>95 \%) \\
\text { (lymphocytes) }\end{array}$ & $\begin{array}{l}\text { Student's t-test; } \\
\text { ANOVA with post-hoc } \\
\text { Tukey’s t-test for } \\
\text { multiple comparisons } \\
\text { during the week; } \\
\text { Pearson coefficient for } \\
\text { age and exposure } \\
\text { duration }\end{array}$ & $\begin{array}{l}\text { Significantly higher no. } \\
\text { of cells with MN and } \\
\text { DNA damage index } \\
\text { compared to unexposed } \\
\text { subjects; age was a } \\
\text { significant factor for } \\
\text { MN; positive } \\
\text { correlation between } \\
\text { DNA damage index and } \\
\text { sampling day such that } \\
\text { the it was significantly } \\
\text { greater on Wednesday } \\
\text { or Thursday relative to } \\
\text { Monday (the first day } \\
\text { sampled); alcohol } \\
\text { consumption also a } \\
\text { significant factor for } \\
\text { DNA damage index }\end{array}$ \\
\hline
\end{tabular}




\begin{tabular}{|c|c|c|c|c|c|c|}
\hline Study & $\begin{array}{l}\text { Study Design } \\
\text { (Occupation, } \\
\text { Location, Sex) }\end{array}$ & No. of Participants & $\begin{array}{c}\text { Exposure Measure } \\
\text { (Duration) }\end{array}$ & Outcome Measured & Analysis & Results \\
\hline Roth et al. (1994) & $\begin{array}{l}\text { Cohort, prospective } \\
\text { (Pharmacists, Finland, } \\
\text { F) }\end{array}$ & $\begin{array}{l}6 \text { exposed: tested at } \\
\text { start of job and after } \\
1 \text { year of work, } 6 \\
\text { unexposed office } \\
\text { workers }\end{array}$ & $\begin{array}{l}\text { Job title, previous and } \\
\text { current drug handling, } \\
\text { use of PPE [method } \\
\text { NR] (duration not } \\
\text { applicable) } \\
\text { Note: Pharmacists used } \\
\text { overall, masks, and } \\
\text { gloves; drug handling } \\
\text { conducted in vertical } \\
\text { laminar flow safety } \\
\text { cabinet in a special } \\
\text { negative-pressured } \\
\text { laboratory, apart from } \\
\text { other working facilities }\end{array}$ & $\begin{array}{l}\% \text { cells with CA and } \\
\text { no. of cells with } \\
\text { MN/1,000 cells scored } \\
\text { (lymphocytes) }\end{array}$ & $\begin{array}{l}\text { Logistic-binomial } \\
\text { regression ( } 30 \text { trials); } \\
\text { model considered } \\
\text { exposure, age, and } \\
\text { sample batch }\end{array}$ & $\begin{array}{l}\text { No difference in \% } \\
\text { cells with CA or no. of } \\
\text { cells with MN after } \\
1 \text { year of work } \\
\text { compared to unexposed } \\
\text { subjects }\end{array}$ \\
\hline Rubeš et al. (1998) & $\begin{array}{l}\text { Cross-sectional } \\
\text { (Nurses and physicians, } \\
\text { Czech Republic, FM) }\end{array}$ & $\begin{array}{l}10 \text { exposed, } 10 \\
\text { unexposed nurses and } \\
\text { doctors, } 11 \text { unexposed } \\
\text { blood donors }\end{array}$ & $\begin{array}{l}\text { Job title and drug } \\
\text { handling (types, } \\
\text { frequency, and } \\
\text { duration) [method NR] } \\
\text { (4-27 years) }\end{array}$ & $\begin{array}{l}\text { \% cells with CA } \\
\text { (lymphocytes) }\end{array}$ & Mann-Whitney U-test & $\begin{array}{l}\text { Significantly higher \% } \\
\text { cells with CA } \\
\text { compared to unexposed } \\
\text { blood donors, but not to } \\
\text { unexposed nurses and } \\
\text { physicians; } \\
\text { significantly higher \% } \\
\text { cells with CA in all } \\
\text { medical personal } \\
\text { compared to unexposed } \\
\text { blood donors; exposure } \\
\text { duration or smoking } \\
\text { status NS }\end{array}$ \\
\hline
\end{tabular}




\begin{tabular}{|c|c|c|c|c|c|c|}
\hline Study & $\begin{array}{l}\text { Study Design } \\
\text { (Occupation, } \\
\text { Location, Sex) }\end{array}$ & No. of Participants & $\begin{array}{c}\text { Exposure Measure } \\
\text { (Duration) }\end{array}$ & Outcome Measured & Analysis & Results \\
\hline Sasaki et al. (2008) & $\begin{array}{l}\text { Cross-sectional } \\
\text { (Nurses, Japan, F) }\end{array}$ & $\begin{array}{l}121 \text { exposed (only } 57 \\
\text { prepared and } \\
\text { administered agents), } \\
46 \text { unexposed hospital } \\
\text { clerks }\end{array}$ & $\begin{array}{l}\text { Job title and self- } \\
\text { reported questionnaire } \\
\text { ( } \leq 6 \text { months) }\end{array}$ & $\begin{array}{l}\text { Tail length and tail } \\
\text { moment (lymphocytes) }\end{array}$ & $\begin{array}{l}\text { ANCOVA tested for } \\
\text { influence of exposure, } \\
\text { sex, age, smoking } \\
\text { habits, occupation, } \\
\text { exposure duration, and } \\
\text { PPE; logarithmic- } \\
\text { transformation because } \\
\text { data were not normally } \\
\text { distributed }\end{array}$ & $\begin{array}{l}\text { Following log- } \\
\text { transformation, } \\
\text { significantly greater tail } \\
\text { length in all } 121 \text { nurses } \\
\text { and in the } 57 \text { actively } \\
\text { exposed nurses } \\
\text { compared to unexposed } \\
\text { clerks; however, no } \\
\text { difference in tail length } \\
\text { of } 64 \text { nurses not } \\
\text { actively exposed } \\
\text { compared to unexposed } \\
\text { clerks or the } 57 \\
\text { exposed nurses; tail } \\
\text { moment NS }\end{array}$ \\
\hline
\end{tabular}




\begin{tabular}{|c|c|c|c|c|c|c|}
\hline Study & $\begin{array}{l}\text { Study Design } \\
\text { (Occupation, } \\
\text { Location, Sex) }\end{array}$ & No. of Participants & $\begin{array}{c}\text { Exposure Measure } \\
\text { (Duration) }\end{array}$ & Outcome Measured & Analysis & Results \\
\hline \multirow[t]{2}{*}{ Sessink et al. (1994a) } & $\begin{array}{l}\text { Cross-sectional } \\
\text { (Nurses and pharmacy } \\
\text { technicians, the } \\
\text { Netherlands, F) }\end{array}$ & $\begin{array}{l}17 \text { exposed, } 35 \\
\text { unexposed pharmacy } \\
\text { technicians }\end{array}$ & $\begin{array}{l}\text { Job title, [self-reported] } \\
\text { questionnaire, and } \\
\text { biomonitoring ( } 7 \text { years, } \\
\text { range } 4-14 \text { years) } \\
\text { Note: Most workers } \\
\text { wore PPE; drugs were } \\
\text { prepared in laminar } \\
\text { down-flow hoods }\end{array}$ & $\begin{array}{l}\text { \% cells with CA } \\
\text { (lymphocytes) }\end{array}$ & $\begin{array}{l}\text { Two-way ANOVA } \\
\text { evaluated exposure and } \\
\text { smoking }\end{array}$ & $\begin{array}{l}\text { No difference in \% } \\
\text { cells with CA } \\
\text { compared to unexposed } \\
\text { subject }\end{array}$ \\
\hline & $\begin{array}{l}\text { Cross-sectional } \\
\text { (Nurses, laboratory } \\
\text { technicians, cleaning } \\
\text { staff, Czech Republic, } \\
\text { FM) }\end{array}$ & $\begin{array}{l}11 \text { exposed, } 23 \\
\text { unexposed physicians, } \\
\text { nurses, lab technicians } \\
\text { and a cleaning woman }\end{array}$ & $\begin{array}{l}\text { Job title, [self-reported] } \\
\text { questionnaire, and } \\
\text { biomonitoring } \\
\text { (13 years, range 3- } \\
25 \text { years) } \\
\text { Note: Most workers } \\
\text { wore PPE; drugs were } \\
\text { prepared in laminar } \\
\text { down-flow hood }\end{array}$ & $\begin{array}{l}\text { \% cells with CA } \\
\text { (lymphocytes) }\end{array}$ & $\begin{array}{l}\text { Two-way ANOVA } \\
\text { evaluated exposure and } \\
\text { smoking }\end{array}$ & $\begin{array}{l}\text { Significantly higher \% } \\
\text { cells with CA } \\
\text { compared to unexposed } \\
\text { workers; additive } \\
\text { effects between } \\
\text { exposure and smoking }\end{array}$ \\
\hline \multirow[t]{2}{*}{ Sorsa et al. (1988) } & $\begin{array}{l}\text { Cross-sectional (Drug } \\
\text { manufacturing workers } \\
\text { and laboratory } \\
\text { technicians working } \\
\text { with cyclophosphamide } \\
\text { (CP), Finland, FM) }\end{array}$ & $\begin{array}{l}36 \text { exposed (13 } \\
\text { manufacturing workers, } \\
3 \text { laboratory } \\
\text { technicians), } 20 \\
\text { unexposed ( } 10 \\
\text { unexposed factory } \\
\text { employees, } 10 \text { controls } \\
\text { from outside the } \\
\text { factory) }\end{array}$ & $\begin{array}{l}\text { Job title, work tasks, } \\
\text { use of PPE [method } \\
\text { NR], and air monitoring } \\
\text { (range } 1.5 \text {-16 years } \\
\text { based on subjects with } \\
\text { MN data) } \\
\text { Note: PPE and best } \\
\text { available safety } \\
\text { techniques were used; } \\
\text { during the preparation } \\
\text { of CP powder, } \\
\text { manufacturing and } \\
\text { production workers } \\
\text { wore gas-protective } \\
\text { suits }\end{array}$ & $\begin{array}{l}\% \text { cells with CA and } \\
\text { no. of CA } / 100 \text { cells } \\
\text { (lymphocytes) }\end{array}$ & NR & $\begin{array}{l}\text { No difference in \% } \\
\text { cells with CA or no. of } \\
\text { CA between exposed } \\
\text { subjects and controls }\end{array}$ \\
\hline & $\begin{array}{l}\text { Cross-sectional } \\
\text { (Drug production and } \\
\text { laboratory workers } \\
\text { working with } \\
\text { cyclophosphamide, } \\
\text { Finland, FM) }\end{array}$ & $\begin{array}{l}21 \text { exposed (12 drug } \\
\text { production workers, } 9 \\
\text { laboratory workers), } 11 \\
\text { unexposed factory } \\
\text { employees }\end{array}$ & & $\begin{array}{l}\text { \% cells with CA and } \\
\text { no. of CA/100 cells } \\
\text { (lymphocytes) }\end{array}$ & NR & $\begin{array}{l}\text { No difference in \% } \\
\text { cells with CA or no. of } \\
\text { CA between exposed } \\
\text { subjects and controls }\end{array}$ \\
\hline
\end{tabular}




\begin{tabular}{|c|c|c|c|c|c|c|}
\hline Study & $\begin{array}{l}\text { Study Design } \\
\text { (Occupation, } \\
\text { Location, Sex) }\end{array}$ & No. of Participants & $\begin{array}{c}\text { Exposure Measure } \\
\text { (Duration) }\end{array}$ & Outcome Measured & Analysis & Results \\
\hline & $\begin{array}{l}\text { Cross-sectional (Same } \\
\text { group of drug } \\
\text { manufacturing and } \\
\text { production workers } \\
\text { working with } \\
\text { cyclophosphamide, } \\
\text { Finland, FM) }\end{array}$ & $\begin{array}{l}19 \text { exposed ( } 7 \text { process } \\
\text { workers, } 12 \text { drug } \\
\text { manufacturing } \\
\text { workers), } 7 \text { unexposed } \\
\text { factory employees }\end{array}$ & & $\begin{array}{l}\text { No. of MN/1,000 cells } \\
\text { (lymphocytes) }\end{array}$ & NR & $\begin{array}{l}\text { No difference in the no. } \\
\text { of } \mathbf{M N} \text { compared to } \\
\text { unexposed employees; } \\
\text { significant correlation } \\
\text { with age }\end{array}$ \\
\hline Stücker et al. (1986) & $\begin{array}{l}\text { Cross-sectional (Nurses } \\
\text { in pneumology, France, } \\
\text { F) }\end{array}$ & $\begin{array}{l}16 \text { exposed, } 24 \\
\text { unexposed hospital } \\
\text { staff from same } \\
\text { department }\end{array}$ & $\begin{array}{l}\text { Job title and [self- } \\
\text { reported questionnaire] } \\
\text { ( } 3.5 \text { years) } \\
\text { Note: PPE not } \\
\text { consistently used, no } \\
\text { vertical laminar flow } \\
\text { cabinet or isolated } \\
\text { room for drug } \\
\text { preparation was } \\
\text { available }\end{array}$ & $\begin{array}{l}\text { No. of CA/100 cells } \\
\text { (lymphocytes) }\end{array}$ & Chi-squared test & $\begin{array}{l}\text { No difference in no. of } \\
\text { CA compared to } \\
\text { unexposed hospital } \\
\text { staff; correlation with } \\
\text { age and adjustment for } \\
\text { radiodiagnostic } \\
\text { exposure NS; authors } \\
\text { state that nurses had } \\
\text { moderate exposure (11 } \\
\text { perfusion per week) }\end{array}$ \\
\hline Testa et al. (2007) & $\begin{array}{l}\text { Cross-sectional } \\
\text { (Nurses, Italy, FM) }\end{array}$ & $\begin{array}{l}76 \text { exposed, } 72 \\
\text { unexposed blood } \\
\text { donors }\end{array}$ & $\begin{array}{l}\text { Job title and } \\
\text { interviewer-led } \\
\text { questionnaire } \\
(12.2 \pm 7.7 \text { years) } \\
\text { Note: Exposed nurses } \\
\text { were involved in } \\
\text { preparation and } \\
\text { administration of } \\
\text { drugs; they used PPE } \\
\text { (gloves, overalls, and } \\
\text { goggles) and chemical } \\
\text { and biohazard laminar } \\
\text { flow [safety cabinets] }\end{array}$ & $\begin{array}{l}\text { No. of CA/100 cells } \\
\text { (lymphocytes) }\end{array}$ & Mann-Whitney U-test & $\begin{array}{l}\text { Significantly higher no. } \\
\text { of CA compared to } \\
\text { unexposed blood } \\
\text { donors }\end{array}$ \\
\hline
\end{tabular}




\begin{tabular}{|c|c|c|c|c|c|c|}
\hline Study & $\begin{array}{l}\text { Study Design } \\
\text { (Occupation, } \\
\text { Location, Sex) }\end{array}$ & No. of Participants & $\begin{array}{c}\text { Exposure Measure } \\
\text { (Duration) }\end{array}$ & Outcome Measured & Analysis & Results \\
\hline Thiringer et al. (1991) & $\begin{array}{l}\text { Cross-sectional } \\
\text { (Nurses, Sweden, FM) }\end{array}$ & $\begin{array}{l}54 \text { exposed nurses in } \\
\text { Hematology and } \\
\text { Oncology, } 54 \\
\text { unexposed nurses }\end{array}$ & $\begin{array}{l}\text { Job title and self- } \\
\text { reported questionnaire } \\
\text { (duration NR) } \\
\text { Note: 91\% of exposed } \\
\text { nurses always used the } \\
\text { safety hood; all but one } \\
\text { nurse always used } \\
\text { gloves; use of masks } \\
\text { was less consistent }\end{array}$ & $\begin{array}{l}\text { No. of MN/1,000 cells } \\
\text { (lymphocytes) }\end{array}$ & $\begin{array}{l}\text { Wilcoxon signed-rank } \\
\text { test }\end{array}$ & $\begin{array}{l}\text { No difference in the no. } \\
\text { of } \mathbf{M N} \text { compared to } \\
\text { unexposed nurses }\end{array}$ \\
\hline Thulin et al. (1995) & $\begin{array}{l}\text { Cross-sectional (nor- } \\
\text { Nitrogen mustard } \\
\text { production workers, } \\
\text { [Sweden], M) }\end{array}$ & $\begin{array}{l}6 \text { exposed, } 6 \text { unexposed } \\
\text { office workers } \\
\text { Exposed subjects were } \\
\text { sampled before } \\
\text { production, } 1 \text { and } 2 \\
\text { weeks during } \\
\text { production, and } 4 \\
\text { weeks after end of } \\
\text { production }\end{array}$ & $\begin{array}{l}\text { Job title, PPE usage, } \\
\text { and environmental } \\
\text { monitoring (4 years) } \\
\text { Note: Production unit } \\
\text { was in a building } \\
\text { adjacent to the office } \\
\text { with free access } \\
\text { between the two spaces; } \\
\text { all workers wore PPE } \\
\text { with personal fresh-air } \\
\text { devices during } \\
\text { production }\end{array}$ & $\begin{array}{l}\text { No. of CA/100 cells } \\
\text { (lymphocytes) }\end{array}$ & $\begin{array}{l}\text { Student's t-test and } \\
\text { Wilcoxon's test }\end{array}$ & $\begin{array}{l}\text { No difference in no. of } \\
\text { CA compared to } \\
\text { unexposed controls at } \\
\text { any sampling time; no. } \\
\text { of CA in unexposed } \\
\text { office workers were } \\
\text { higher than concurrent } \\
\text { unexposed subjects or } \\
\text { historical unexposed } \\
\text { data }\end{array}$ \\
\hline Ündeğer et al. (1999) & $\begin{array}{l}\text { Cross-sectional } \\
\text { (Nurses, Turkey, FM) }\end{array}$ & $\begin{array}{l}30 \text { exposed, } 30 \\
\text { unexposed nurses, } \\
\text { secretaries, and } \\
\text { technicians }\end{array}$ & $\begin{array}{l}\text { Job title and self- } \\
\text { reported questionnaire } \\
\text { (range } 0.5-13 \text { years) } \\
\text { Note: } 26 \text { exposed } \\
\text { nurses used safety } \\
\text { ventilation cabinets; } 13 \\
\text { used gloves, masks, and } \\
\text { gowns; } 17 \text { did not use } \\
\text { gloves }\end{array}$ & $\begin{array}{l}\text { DNA damage index } \\
(2.5=<5 \%, 12.5=5- \\
20 \%, 30=>20-40 \%, \\
67.5=>40-95 \%, \\
97.5=>95 \%)\end{array}$ & $\begin{array}{l}\text { Student's t-test; linear } \\
\text { regression for effects of } \\
\text { exposure duration }\end{array}$ & $\begin{array}{l}\text { Significantly greater } \\
\text { DNA damage index } \\
\text { of }>5 \% \text { damage } \\
\text { compared to unexposed } \\
\text { subjects; no correlation } \\
\text { with age, exposure } \\
\text { duration; exposed } \\
\text { nurses without safety } \\
\text { equipment had } \\
\text { significantly higher } \\
\text { DNA damage index } \\
\text { than those with safety } \\
\text { equipment }\end{array}$ \\
\hline
\end{tabular}




\begin{tabular}{|c|c|c|c|c|c|c|}
\hline Study & $\begin{array}{l}\text { Study Design } \\
\text { (Occupation, } \\
\text { Location, Sex) }\end{array}$ & No. of Participants & $\begin{array}{c}\text { Exposure Measure } \\
\text { (Duration) }\end{array}$ & Outcome Measured & Analysis & Results \\
\hline Ursini et al. (2006) & $\begin{array}{l}\text { Cross-sectional (Nurses } \\
\text { and pharmacy } \\
\text { technicians, Italy, FM) } \\
\text { Note: Same population } \\
\text { as Cavallo } 2005\end{array}$ & $\begin{array}{l}30 \text { exposed ( } 5 \\
\text { pharmacy technicians, } \\
15 \text { day nurses, } 10 \text { ward } \\
\text { nurses), } 30 \text { unexposed } \\
\text { administrative staff }\end{array}$ & $\begin{array}{l}\text { Job title, self-reported } \\
\text { questionnaire, } \\
\text { biomonitoring, and } \\
\text { environmental } \\
\text { monitoring } \\
\text { (6.2 } 2.9 \text { years, ward } \\
\text { nurses; } 7.0 \pm 2.0 \text { years, } \\
\text { pharmacists; } \\
8.1 \pm 6.0 \text { years, } \\
\text { outpatient nurses }) \\
\text { Note: Exposed } \\
\text { personnel wore PPE, } \\
\text { including gloves, caps, } \\
\text { overalls, and goggles; } \\
\text { specialized pharmacy } \\
\text { prepared the doses }\end{array}$ & $\begin{array}{l}\text { Tail moment } \\
\text { (lymphocytes or buccal } \\
\text { cells) }\end{array}$ & $\begin{array}{l}\text { Student's t-test; } \\
\text { ANOVA to test effects } \\
\text { of age and exposure } \\
\text { duration }\end{array}$ & $\begin{array}{l}\text { No difference in tail } \\
\text { moment (in either cell } \\
\text { population) compared } \\
\text { to unexposed subjects }\end{array}$ \\
\hline Villarini et al. (2011) & $\begin{array}{l}\text { Cross-sectional } \\
\text { (Pharmacy technicians, } \\
\text { nurses, and nurses' } \\
\text { aides, Italy, FM) } \\
\text { Note: Same population } \\
\text { as Moretti } 2013\end{array}$ & $\begin{array}{l}52 \text { exposed subjects } \\
\text { involved in preparation, } \\
\text { administration, } \\
\text { transportation, and } \\
\text { disposal, } 52 \text { unexposed } \\
\text { public workers from } \\
\text { same localities as } \\
\text { exposed }\end{array}$ & $\begin{array}{l}\text { Job title, interviewer- } \\
\text { led questionnaire, } \\
\text { biomonitoring, and } \\
\text { environmental } \\
\text { monitoring ( }<10 \text { years, } \\
\mathrm{n}=13 ; 11-20 \text { years, } \\
\mathrm{n}=14 \text {, and }>20 \text { years, } \\
\mathrm{n}=4 \text { ) } \\
\text { Note: Exposed workers } \\
\text { involved in preparation, } \\
\text { transportation, } \\
\text { administration, and } \\
\text { disposal of cancer } \\
\text { chemotherapy agents }\end{array}$ & $\begin{array}{l}\text { \% Tail DNA and tail } \\
\text { length } \\
\text { (leukocytes) }\end{array}$ & $\begin{array}{l}\text { Mann-Whitney U-test; } \\
\text { sub-group analysis by } \\
\text { Kruskal-Wallis H-test; } \\
\text { multivariate regression } \\
\text { analysis to examine } \\
\text { effect of exposure } \\
\text { status, gender, age, } \\
\text { smoking, occupation, } \\
\text { PPE, job seniority, and } \\
\text { genetic polymorphisms }\end{array}$ & $\begin{array}{l}\text { Significantly greater \% } \\
\text { tail DNA in exposed } \\
\text { subjects; tail length } \\
\text { NS; occupation was a } \\
\text { significant covariate }\end{array}$ \\
\hline Yager et al. (1988) & $\begin{array}{l}\text { Cross-sectional } \\
\text { (Chemical process } \\
\text { workers, manufacturing } \\
\text { workers, and nurses, } \\
\text { Finland, FM) } \\
\text { Note: Overlapping } \\
\text { population with Sorsa } \\
1988\end{array}$ & $\begin{array}{l}27 \text { exposed ( } 7 \text { chemical } \\
\text { process workers, } 12 \\
\text { manufacturing workers, } \\
\text { and } 8 \text { oncology nurses), } \\
9 \text { unexposed subjects } \\
\text { [no description } \\
\text { provided] }\end{array}$ & $\begin{array}{l}\text { Job title and work } \\
\text { history [method NR] } \\
\text { (range 2-25 years) }\end{array}$ & $\begin{array}{l}\text { No. of MN/1,000 cells } \\
\text { (lymphocytes) }\end{array}$ & $\begin{array}{l}\text { ANOVA; log- } \\
\text { transformation of data } \\
\text { and adjustment for } \\
\text { influence of age }\end{array}$ & $\begin{array}{l}\text { ANOVA variables for } \\
\text { exposure and age were } \\
\text { significant); log- } \\
\text { transformed data } \\
\text { adjusted for age } \\
\text { revealed significantly } \\
\text { higher no. of MN } \\
\text { compared to unexposed } \\
\text { subjects }\end{array}$ \\
\hline
\end{tabular}




\begin{tabular}{|c|c|c|c|c|c|c|}
\hline Study & $\begin{array}{l}\text { Study Design } \\
\text { (Occupation, } \\
\text { Location, Sex) }\end{array}$ & No. of Participants & $\begin{array}{c}\text { Exposure Measure } \\
\text { (Duration) }\end{array}$ & Outcome Measured & Analysis & Results \\
\hline Yang et al. (2002a) & $\begin{array}{l}\text { Cross-sectional } \\
\text { (Nurses, China, [F]) } \\
\text { Note: Same population } \\
\text { as Yang 2002b }\end{array}$ & $\begin{array}{l}16 \text { exposed, } 16 \text { nursing } \\
\text { students }\end{array}$ & $\begin{array}{l}\text { Job title and drug } \\
\text { handling (types and } \\
\text { frequency) ( } 5.6 \text { years, } \\
\text { range } 1-16 \text { years) }\end{array}$ & Tail length & [Mann-Whitney] U-test & $\begin{array}{l}\text { Significantly greater } \\
\text { tail length in exposed } \\
\text { subjects than controls }\end{array}$ \\
\hline Yang et al. (2002b) & $\begin{array}{l}\text { Cross-sectional } \\
\text { (Nurses, China, F) } \\
\text { Note: Same population } \\
\text { as Yang 2002a }\end{array}$ & $\begin{array}{l}16 \text { exposed, } 16 \text { nursing } \\
\text { students }\end{array}$ & $\begin{array}{l}\text { Job title and frequency } \\
\text { of drug handling } \\
\text { (5.5 years) }\end{array}$ & $\begin{array}{l}\text { No. of CA/100 cells } \\
\text { and no. of MN/1,000 } \\
\text { cells (lymphocytes) }\end{array}$ & [Mann-Whitney U-test] & $\begin{array}{l}\text { Significantly higher no. } \\
\text { of CA and no. of } \mathbf{M N} \\
\text { compared to unexposed } \\
\text { nursing students }\end{array}$ \\
\hline Yin et al. (2010) & $\begin{array}{l}\text { Cross-sectional } \\
\text { (Oncology Nurses, } \\
\text { China, F) }\end{array}$ & $\begin{array}{l}30 \text { exposed, } 30 \\
\text { unexposed nurses }\end{array}$ & $\begin{array}{l}\text { Job title, work tasks, } \\
\text { drug handling, PPE } \\
\text { usage [method NR] } \\
\text { (9.8 } \pm 5.9 \text { years) } \\
\text { Note: PPE and } \\
\text { biosafety cabinets were } \\
\text { used during } \\
\text { preparation of doses }\end{array}$ & $\begin{array}{l}\% \text { Tail DNA, tail } \\
\text { length, and tail moment }\end{array}$ & $\begin{array}{l}\text { Logarithmic- } \\
\text { transformation followed } \\
\text { by multiple linear } \\
\text { regression }\end{array}$ & $\begin{array}{l}\text { Significantly greater \% } \\
\text { tail DNA, tail length, } \\
\text { and tail moment } \\
\text { compared to unexposed } \\
\text { nurses }\end{array}$ \\
\hline Yoshida et al. (2006) & $\begin{array}{l}\text { Cross-sectional (Nurses } \\
\text { from hematology } \\
\text { department, Japan, F) }\end{array}$ & $\begin{array}{l}19 \text { exposed nurses, } 18 \\
\text { unexposed nurses from } \\
\text { Department of } \\
\text { Cardiovascular Disease }\end{array}$ & $\begin{array}{l}\text { Job title and self- } \\
\text { reported questionnaire } \\
\text { (5.7 years, range } 0.2- \\
25.2 \text { years) }\end{array}$ & $\begin{array}{l}\text { Tail length } \\
\text { (lymphocytes) }\end{array}$ & Mann-Whitney U-test & $\begin{array}{l}\text { Significantly greater } \\
\text { tail length compared to } \\
\text { unexposed nurses; age } \\
\text { and duration of } \\
\text { exposure were } \\
\text { significant variables }\end{array}$ \\
\hline
\end{tabular}




\title{
Appendix G. List of Included Studies Reporting on Environmental Monitoring or Biomonitoring
}

\author{
Acampora A, Castiglia L, Miraglia N, Pieri M, Soave C, Liotti F, Sannolo N. 2005. A case \\ study: Surface contamination of cyclophosphamide due to working practices and cleaning \\ procedures in two Italian hospitals. Ann Occup Hyg. 49(7):611-618. \\ http://dx.doi.org/10.1093/annhyg/mei029
}

Azari M, Panahi D, Akbari ME, Mirzaei HR, Rezvani HR, Zendehdel R, Mehrabi Y, Bayatian M. 2017. Environmental monitoring of occupational exposure to cyclophosphamide drug in two Iranian hospitals. Iran J Cancer Prev. 10(1). http://dx.doi.org/10.17795/ijcp-7229

Berruyer M, Tanguay C, Caron NJ, Lefebvre M, Bussières JF. 2015. Multicenter study of environmental contamination with antineoplastic drugs in 36 Canadian Hospitals: A 2013 follow-up study. J Occup Environ Hyg. 12(2):87-94.

http://dx.doi.org/10.1080/15459624.2014.949725

Bigelow S, Schulz H, Dobish R, Chambers CR. 2009. Antineoplastic agent workplace contamination study: The Alberta Cancer Board Pharmacy perspective Phase III. J Oncol Pharm Pract. 15(3):157-160. http://dx.doi.org/10.1177/1078155208101097

Bobin-Dubigeon C, Amiand M, Percheron C, Audeval C, Rochard S, Leynia P, Bard JM. 2013. A new, validated wipe-sampling procedure coupled to LC-MS analysis for the simultaneous determination of 5-fluorouracil, doxorubicin and cyclophosphamide in surface contamination. $\mathrm{J}$ Anal Toxicol. 37(7):433-439. http://dx.doi.org/10.1093/jat/bkt045

Bolognesi C, Nucci MC, Colacci AM, Grilli S, Ippoliti F, Mucci N, Romano Spica V, Barbieri A, Canitano N, Chiozzotto D et al. 2005. [Biomonitoring of nurses occupationally exposed to antineoplastic drugs: The IMEPA Project]. Epidemiol Prev. 29(5-6 Suppl):91-95.

Bos RP, Weissenberger BFJ, Anzion RBM. 1998. $\alpha$-Fluoro- $\beta$-alanine in urine of workers occupationally exposed to 5- fluorouracil in a 5-fluorouracil-producing factory. Biomarkers. 3(1):81-87. http://dx.doi.org/10.1080/135475098231390

Burgaz S, Karahalil B, Bayrak P, Taskin L, Yavuzaslan F, Bokesoy I, Anzion RB, Bos RP, Platin N. 1999. Urinary cyclophosphamide excretion and micronuclei frequencies in peripheral lymphocytes and in exfoliated buccal epithelial cells of nurses handling antineoplastics. Mutat Res. 439(1):97-104. http://dx.doi.org/10.1016/S1383-5718(98)00180-6

Burgaz S, Karahalil B, Canhi Z, Terzioglu F, Ancel G, Anzion RB, Bos RP, Huttner E. 2002. Assessment of genotoxic damage in nurses occupationally exposed to antineoplastics by the analysis of chromosomal aberrations. Hum Exp Toxicol. 21(3):129-135.

http://dx.doi.org/10.1191/0960327102ht230oa

Bussières JF, Tanguay C, Touzin K, Langlois E, Lefebvre M. 2012. Environmental contamination with hazardous drugs in Quebec hospitals. Can J Hosp Pharm. 65(6):428-435. http://dx.doi.org/10.4212/cjhp.v65i6.1190 
Canal-Raffin M, Khennoufa K, Martinez B, Goujon Y, Folch C, Ducint D, Titier K, Brochard P, Verdun-Esquer C, Molimard M. 2016. Highly sensitive LC-MS/MS methods for urinary biological monitoring of occupational exposure to cyclophosphamide, ifosfamide, and methotrexate antineoplastic drugs and routine application. J Chromatogr B. 1038:109-117. http://dx.doi.org/10.1016/j.jchromb.2016.10.021

Caneparo A, Massucco P, Vaira M, Maina G, Giovale E, Coggiola M, Cinquegrana A, Robella M, De Simone M. 2014. Contamination risk for operators performing semi-closed HIPEC procedure using cisplatin. Eur J Surg Oncol. 40(8):925-929.

http://dx.doi.org/10.1016/j.ejso.2014.03.013

Caporossi L, De Rosa M, Pera A, Papaleo B. 2007. Simple analytical method for the determination of paclitaxel (Taxol ${ }^{\circledR}$ ) levels in human plasma. Chromatographia. 66(11-12):921924. http://dx.doi.org/10.1365/s10337-007-0427-z

Castiglia L, Miraglia N, Pieri M, Simonelli A, Basilicata P, Genovese G, Guadagni R, Acampora A, Sannolo N, Scafarto MV. 2008. Evaluation of occupational exposure to antiblastic drugs in an Italian hospital oncological department. J Occup Health. 50(1):48-56.

http://dx.doi.org/10.1539/joh.50.48

Cavallo D, Ursini CL, Perniconi B, Francesco AD, Giglio M, Rubino FM, Marinaccio A, Iavicoli S. 2005. Evaluation of genotoxic effects induced by exposure to antineoplastic drugs in lymphocytes and exfoliated buccal cells of oncology nurses and pharmacy employees. Mutat Res. 587(1-2):45-51. http://dx.doi.org/10.1016/j.mrgentox.2005.07.008

Chu WC, Hon CY, Danyluk Q, Chua PP, Astrakianakis G. 2012. Pilot assessment of the antineoplastic drug contamination levels in British Columbian hospitals pre- and post-cleaning. J Oncol Pharm Pract. 18(1):46-51. http://dx.doi.org/10.1177/1078155211402106

Clark BA, Sessink PJ. 2013. Use of a closed system drug-transfer device eliminates surface contamination with antineoplastic agents. J Oncol Pharm Pract. 19(2):99-104. http://dx.doi.org/10.1177/1078155212468367

Connor TH, Anderson RW, Sessink PJ, Broadfield L, Power LA. 1999. Surface contamination with antineoplastic agents in six cancer treatment centers in Canada and the United States. Am J Health Syst Pharm. 56(14):1427-1432.

Connor TH, Anderson RW, Sessink PJ, Spivey SM. 2002. Effectiveness of a closed-system device in containing surface contamination with cyclophosphamide and ifosfamide in an i.v. admixture area. Am J Health Syst Pharm. 59(1):68-72.

Connor TH, DeBord DG, Pretty JR, Oliver MS, Roth TS, Lees PS, Krieg EF, Jr., Rogers B, Escalante CP, Toennis CA et al. 2010. Evaluation of antineoplastic drug exposure of health care workers at three university-based US cancer centers. J Occup Environ Med. 52(10):1019-1027. http://dx.doi.org/10.1097/JOM.0b013e3181f72b63

Connor TH, Sessink PJ, Harrison BR, Pretty JR, Peters BG, Alfaro RM, Bilos A, Beckmann G, Bing MR, Anderson LM et al. 2005. Surface contamination of chemotherapy drug vials and evaluation of new vial-cleaning techniques: Results of three studies. Am J Health Syst Pharm. 62(5):475-484. http://dx.doi.org/10.1093/ajhp/62.5.475 
Couch J, dePerio MA. 2011. Health Hazard Evaluation Report: HETA-2010-0118-3142, September 2011. Chemotherapy drug evaluation at a medical laboratory - Pennsylvania. Govt Reports Announcements \& Index. (04):24.

Couch J, Gibbins J, Connor T. 2012. Health Hazard Evaluation Report: HETA-2010-0068-3156, April 2012. Chemotherapy drug evaluation at a veterinary teaching hospital - Michigan. Govt Reports Announcements \& Index. (15):32.

Couch J, West C. 2012. Health Hazard Evaluation Report: HETA-2009-0148-3158, June 2012. Chemotherapy drug exposure at an oncology clinic - Florida. Govt Reports Announcements \& Index. (20):36.

Crauste-Manciet S, Sessink PJ, Ferrari S, Jomier JY, Brossard D. 2005. Environmental contamination with cytotoxic drugs in healthcare using positive air pressure isolators. Ann Occup Hyg. 49(7):619-628. http://dx.doi.org/10.1093/annhyg/mei045

Crickman R. 2017. Chemotherapy safe handling: Limiting nursing exposure with a hazardous drug control program. Clin J Oncol Nurs. 21(1):73-78. http://dx.doi.org/10.1188/17.cjon.73-78

Deschamps F, Lesage FX, Marinutti-Liberge V, Lamiable D, Millart H. 2007. Letter to the editor. Inhal Toxicol. 19(3):309. http://dx.doi.org/10.1080/08958370601069422

deWerk Neal A, Wadden RA, Chiou WL. 1983. Exposure of hospital workers to airborne antineoplastic agents. Am J Hosp Pharm. 40(4):597-601.

Ensslin AS, Huber R, Pethran A, Rommelt H, Schierl R, Kulka U, Fruhmann G. 1997. Biological monitoring of hospital pharmacy personnel occupationally exposed to cytostatic drugs: Urinary excretion and cytogenetics studies. Int Arch Occup Environ Health. 70(3):205208. http://dx.doi.org/10.1007/s004200050208

Ensslin AS, Pethran A, Schierl R, Fruhmann G. 1994. Urinary platinum in hospital personnel occupationally exposed to platinum-containing antineoplastic drugs. Int Arch Occup Environ Health. 65(5):339-342. http://dx.doi.org/10.1007/BF00405699

Ensslin AS, Stoll Y, Pethran A, Pfaller A, Rommelt H, Fruhmann G. 1994. Biological monitoring of cyclophosphamide and ifosfamide in urine of hospital personnel occupationally exposed to cytostatic drugs. Occup Environ Med. 51(4):229-233.

http://dx.doi.org/10.1136/oem.51.4.229

Evelo CT, Bos RP, Peters JG, Henderson PT. 1986. Urinary cyclophosphamide assay as a method for biological monitoring of occupational exposure to cyclophosphamide. Int Arch Occup Environ Health. 58(2):151-155. http://dx.doi.org/10.1007/BF00380766

Favier B, Gilles L, Ardiet C, Latour JF. 2003. External contamination of vials containing cytotoxic agents supplied by pharmaceutical manufacturers. J Oncol Pharm Pract. 9(1):15-20. http://dx.doi.org/10.1191/1078155203jp102oa

Fent KW, Durgam S, Mueller C. 2014. Pharmaceutical dust exposure at pharmacies using automatic dispensing machines: A preliminary study. J Occup Environ Hyg. 11(11):695-705. http://dx.doi.org/10.1080/15459624.2014.918983 
Fleury-Souverain S, Mattiuzzo M, Mehl F, Nussbaumer S, Bouchoud L, Falaschi L, Gex-Fabry M, Rudaz S, Sadeghipour F, Bonnabry P. 2015. Evaluation of chemical contamination of surfaces during the preparation of chemotherapies in 24 hospital pharmacies. Eur J Hosp Pharm. 22(6):333-341. http://dx.doi.org/10.1136/ejhpharm-2014-000549

Fleury-Souverain S, Nussbaumer S, Mattiuzzo M, Bonnabry P. 2014. Determination of the external contamination and cross-contamination by cytotoxic drugs on the surfaces of vials available on the Swiss market. J Oncol Pharm Pract. 20(2):100-111.

http://dx.doi.org/10.1177/1078155213482683

Floridia L, Pietropaolo AM, Tavazzani M, Rubino FM, Colombi A. 1999. High-performance liquid chromatography of methotrexate for environmental monitoring of surface contamination in hospital departments and assessment of occupational exposure. J Chromatogr B Biomed Sci Appl. 726(1-2):95-103. http://dx.doi.org/10.1016/S0378-4347(98)00561-1

Fransman W, Huizer D, Tuerk J, Kromhout H. 2007. Inhalation and dermal exposure to eight antineoplastic drugs in an industrial laundry facility. Int Arch Occup Environ Health. 80(5):396403. http://dx.doi.org/10.1007/s00420-006-0148-x

Fransman W, Peelen S, Hilhorst S, Roeleveld N, Heederik D, Kromhout H. 2007. A pooled analysis to study trends in exposure to antineoplastic drugs among nurses. Ann Occup Hyg. 51(3):231-239. http://dx.doi.org/10.1093/annhyg/mel081

Fransman W, Roeleveld N, Peelen S, de Kort W, Kromhout H, Heederik D. 2007. Nurses with dermal exposure to antineoplastic drugs: Reproductive outcomes. Epidemiology. 18(1):112-119. http://dx.doi.org/10.1097/01.ede.0000246827.44093.c

Fransman W, Vermeulen R, Kromhout H. 2004. Occupational dermal exposure to cyclophosphamide in Dutch hospitals: A pilot study. Ann Occup Hyg. 48(3):237-244. http://dx.doi.org/10.1093/annhyg/meh017

Fransman W, Vermeulen R, Kromhout H. 2005. Dermal exposure to cyclophosphamide in hospitals during preparation, nursing and cleaning activities. Int Arch Occup Environ Health. 78(5):403-412. http://dx.doi.org/10.1007/s00420-004-0595-1

Friederich U, Molko F, Hofmann V, Scossa D, Hann D, Wurgler FE, Senn HJ. 1986. Limitations of the salmonella/mammalian microsome assay (Ames test) to determine occupational exposure to cytostatic drugs. Eur J Cancer Clin Oncol. 22(5):567-575. http://dx.doi.org/10.1016/0277$\underline{5379(86) 90045-3}$

Friese CR, McArdle C, Zhao T, Sun D, Spasojevic I, Polovich M, McCullagh MC. 2015. Antineoplastic drug exposure in an ambulatory setting: A pilot study. Cancer Nurs. 38(2):111117. http://dx.doi.org/10.1097/NCC.0000000000000143

Fung V, Seneviratne M. 2016. Regulatory verification on safe use of cytotoxic drugs in veterinary clinics and animal hospitals. Aust Vet J. 94(11):400-404. http://dx.doi.org/10.1111/avj.12515 
Graeve C, McGovern PM, Arnold S, Polovich M. 2017. Testing an intervention to decrease healthcare workers' exposure to antineoplastic agents. Oncol Nurs Forum. 44(1):E10-e19. http://dx.doi.org/10.1188/17.onf.e10-e19

Guillemette A, Langlois H, Voisine M, Merger D, Therrien R, Mercier G, Lebel D, Bussieres JF. 2014. Impact and appreciation of two methods aiming at reducing hazardous drug environmental contamination: The centralization of the priming of IV tubing in the pharmacy and use of a closed-system transfer device. J Oncol Pharm Pract. 20(6):426-432.

http://dx.doi.org/10.1177/1078155213517127

Guinée EP, Beuman GH, Hageman G, Welle IJ, Kleinjans JC. 1991. Evaluation of genotoxic risk of handling cytostatic drugs in clinical pharmacy practice. Pharm Weekbl Sci. 13(2):78-82. http://dx.doi.org/10.1007/BF01974985

Hama K, Fukushima K, Hirabatake M, Hashida T, Kataoka K. 2012. Verification of surface contamination of Japanese cyclophosphamide vials and an example of exposure by handling. J Oncol Pharm Pract. 18(2):201-206. http://dx.doi.org/10.1177/1078155211419543

Hama T, Aoyama T, Shirai T, Higuchi S, Yokokawa T, Sugimoto Y. 2009. Environmental contamination due to preparation of cyclophosphamide and exposure of pharmacists to it. Jpn J Pharm Health Care Sci. 35(10):693-700. http://dx.doi.org/10.5649/jjphcs.35.693

Hanada T, Takami Y, Moriyama K, Oro M, Ogawa T, Moriyasu H, Inoue Y, Kanemitsu A, Kawamoto E, Nagase A et al. 2015. Prevention of the exposure by cyclophosphamide oral tablet. J Pharm Health Care Sci. 1:20. http://dx.doi.org/10.1186/s40780-015-0020-9

Harrison BR, Peters BG, Bing MR. 2006. Comparison of surface contamination with cyclophosphamide and fluorouracil using a closed-system drug transfer device versus standard preparation techniques. Am J Health Syst Pharm. 63(18):1736-1744.

http://dx.doi.org/10.2146/ajhp050258

Hedmer M, Georgiadi A, Bremberg ER, Jonsson BA, Eksborg S. 2005. Surface contamination of cyclophosphamide packaging and surface contamination with antineoplastic drugs in a hospital pharmacy in Sweden. Ann Occup Hyg. 49(7):629-637. http://dx.doi.org/10.1093/annhyg/mei042

Hedmer M, Tinnerberg H, Axmon A, Jonsson BA. 2008. Environmental and biological monitoring of antineoplastic drugs in four workplaces in a Swedish hospital. Int Arch Occup Environ Health. 81(7):899-911. http://dx.doi.org/10.1007/s00420-007-0284-y

Hedmer M, Wohlfart G. 2012. Hygienic guidance values for wipe sampling of antineoplastic drugs in Swedish hospitals. J Environ Monit. 14(7):1968-1975.

http://dx.doi.org/10.1039/c2em10704j

Higginbotham ML, Fritz SE. 2017. Evaluation of environmental cytotoxic drug contamination in a clinical setting. J Am Anim Hosp Assoc. 53(1):32-37. http://dx.doi.org/10.5326/jaaha-ms-6471

Hirst M, Tse S, Mills DG, Levin L, White DF. 1984. Occupational exposure to cyclophosphamide. Lancet. 323(8370):186-188. http://dx.doi.org/10.1016/S0140$\underline{6736(84) 92111-1}$ 
Hon CY, Astrakianakis G, Danyluk Q, Chu W. 2011. Pilot evaluation of dermal contamination by antineoplastic drugs among hospital pharmacy personnel. Can J Hosp Pharm. 64(5):327-332.

Hon CY, Chua PP, Danyluk Q, Astrakianakis G. 2014. Examining factors that influence the effectiveness of cleaning antineoplastic drugs from drug preparation surfaces: A pilot study. J Oncol Pharm Pract. 20(3):210-216. http://dx.doi.org/10.1177/1078155213497070

Hon CY, Teschke K, Chu W, Demers P, Venners S. 2013. Antineoplastic drug contamination of surfaces throughout the hospital medication system in Canadian hospitals. J Occup Environ Hyg. 10(7):374-383. http://dx.doi.org/10.1080/15459624.2013.789743

Hon CY, Teschke K, Chua P, Venners S, Nakashima L. 2011. Occupational exposure to antineoplastic drugs: Identification of job categories potentially exposed throughout the hospital medication system. Saf Health Work. 2(3):273-281.

http://dx.doi.org/10.5491/SHAW.2011.2.3.273

Hon CY, Teschke K, Demers PA, Venners S. 2014. Antineoplastic drug contamination on the hands of employees working throughout the hospital medication system. Ann Occup Hyg. 58(6):761-770. http://dx.doi.org/10.1093/annhyg/meu019

Hon CY, Teschke K, Shen H, Demers PA, Venners S. 2015. Antineoplastic drug contamination in the urine of Canadian healthcare workers. Int Arch Occup Environ Health. 88(7):933-941. http://dx.doi.org/10.1007/s00420-015-1026-1

Ikeda K, Yagi Y, Takegami M, Lu Y, Morimoto K, Kurokawa N. 2007. Efforts to ensure safety of hospital pharmacy personnel occupationally exposed to antineoplastic drugs during a preparation task. Hosp Pharm. 42(3):209-218. http://dx.doi.org/10.1310/hpj4203-209

Janes A, Tanguay C, Caron NJ, Bussieres JF. 2015. Environmental contamination with cyclophosphamide, ifosfamide, and methotrexate: A study of 51 Canadian centres. Can J Hosp Pharm. 68(4):279-289.

Janssens T, Brouwers EE, de Vos JP, de Vries N, Schellens JH, Beijnen JH. 2015. Determination of platinum surface contamination in veterinary and human oncology centres using inductively coupled plasma mass spectrometry. Vet Comp Oncol. 13(3):305-313.

http://dx.doi.org/10.1111/vco.12049

Jeebhay M, Mbuli S, Uebel R. 1993. Assessment of exposure to chloramphenicol and azathioprine among workers in a South African pharmaceutical plant. Int Arch Occup Environ Health. 65(S1):S119-122. http://dx.doi.org/10.1007/BF00381321

Johnson BL. 1982. Handling methotrexate --a safety problem? Am J Nurs. 82(10):1531. http://dx.doi.org/10.1097/00000446-198282100-00006

Karahalil B, Ilter Akkoyunlu K. 2004. Determination of urinary cyclophosphamide in oncology nurses handling antineoplastic drugs by gas chromatography-mass spectrometry. FABAD J Pharm Sci. 28(3):125-130.

Kiefer M. 2002. Health Hazard Evaluation Report: HETA 98-0052-2820, MD Anderson Cancer Center, Houston, Texas. Govt Reports Announcements \& Index. (26):28. 
Kiffmeyer TK, Kube C, Opiolka S, Schmidt KG, Schöppe G, Sessink PJM. 2002. Vapour pressures, evaporation behaviour and airborne concentrations of hazardous drugs: Implications for occupational safety. Pharm J. 268(7188):331-337.

Kiffmeyer TK, Tuerk J, Hahn M, Stuetzer H, Hadtstein C, Heinemann A, Eickmann U. 2013. Application and assessment of a regular environmental monitoring of the antineoplastic drug contamination level in pharmacies - the MEWIP project. Ann Occup Hyg. 57(4):444-455. http://dx.doi.org/10.1093/annhyg/mes081

Konate A, Poupon J, Villa A, Garnier R, Hasni-Pichard H, Mezzaroba D, Fernandez G, Pocard M. 2011. Evaluation of environmental contamination by platinum and exposure risks for healthcare workers during a heated intraperitoneal perioperative chemotherapy (HIPEC) procedure. J Surg Oncol. 103(1):6-9. http://dx.doi.org/10.1002/jso.21740

Kopp B, Crauste-Manciet S, Guibert A, Mourier W, Guerrault-Moro MN, Ferrari S, Jomier JY, Brossard D, Schierl R. 2013. Environmental and biological monitoring of platinum-containing drugs in two hospital pharmacies using positive air pressure isolators. Ann Occup Hyg. 57(3):374-383. http://dx.doi.org/10.1093/annhyg/mes073

Kopp B, Schierl R, Nowak D. 2013. Evaluation of working practices and surface contamination with antineoplastic drugs in outpatient oncology health care settings. Int Arch Occup Environ Health. 86(1):47-55. http://dx.doi.org/10.1007/s00420-012-0742-z

Korczowska E, Jankowiak-Gracz H. 2013. How can we improve safe handling of antineoplastic drugs: Can devices be helpful. Eur J Onc Pharm. 7(1):5-7.

Kromhout H, Hoek F, Uitterhoeve R, Huijbers R, Overmars RF, Anzion R, Vermeulen R. 2000. Postulating a dermal pathway for exposure to anti-neoplastic drugs among hospital workers. Applying a conceptual model to the results of three workplace surveys. Ann Occup Hyg. 44(7):551-560. http://dx.doi.org/10.1016/S0003-4878(00)00050-8

Kushnir CL, Fleury AC, Couch J, Hill MC, Spirtos NM. 2013. Evaluation of exposures to healthcare personnel from cisplatin during a mock demonstration of intra-operative intraperitoneal chemotherapy administration. Gynecol Oncol. 130(2):350-353. http://dx.doi.org/10.1016/j.ygyno.2013.04.467

Ladeira C, Viegas S, Pádua M, Gomes M, Carolino E, Gomes MC, Brito M. 2014. Assessment of genotoxic effects in nurses handling cytostatic drugs. J Toxicol Environ Health A. 77(1416):879-887. http://dx.doi.org/10.1080/15287394.2014.910158

Lee SG, Tkaczuk M, Jankewicz G, Ambados F. 2007. Surface contamination from cytotoxic chemotherapy following preparation and administration. J Pharm Pract Res. 37(4):271-276.

Mader RM, Kokalj A, Kratochvil E, Pilger A, Rudiger HW. 2009. Longitudinal biomonitoring of nurses handling antineoplastic drugs. J Clin Nurs. 18(2):263-269.

http://dx.doi.org/10.1111/j.1365-2702.2007.02189.x

Mader RM, Rizovski B, Steger GG, Wachter A, Kotz R, Rainer H. 1996. Exposure of oncologic nurses to methotrexate in the treatment of osteosarcoma. Arch Environ Health. 51(4):310-314. http://dx.doi.org/10.1080/00039896.1996.9936030 
Maeda S, Miyawaki K, Matsumoto S, Oishi M, Miwa Y, Kurokawa N. 2010. Evaluation of environmental contaminations and occupational exposures involved in preparation of chemotherapeutic drugs. Yakugaku Zasshi. 130(6):903-910. http://dx.doi.org/10.1248/yakushi.130.903

Martins I, Apostoli P, Della Rosa HV. 2008. Cyclophosphamide levels in sites of preparation and administration of antineoplastic drugs. Lat Am J Pharm. 27(2):217-223.

Martins I, Rosa HVD, Apostoli P. 2004. Cyclophosphamide identification in wipe test by GCMS and solid phase extraction. Braz J Pharm. 40(1):67-73.

Mason HJ, Blair S, Sams C, Jones K, Garfitt SJ, Cuschieri MJ, Baxter PJ. 2005. Exposure to antineoplastic drugs in two UK hospital pharmacy units. Ann Occup Hyg. 49(7):603-610. http://dx.doi.org/10.1093/annhyg/mei023

Mason HJ, Morton J, Garfitt SJ, Iqbal S, Jones K. 2003. Cytotoxic drug contamination on the outside of vials delivered to a hospital pharmacy. Ann Occup Hyg. 47(8):681-685.

McDevitt JJ, Lees PS, McDiarmid MA. 1993. Exposure of hospital pharmacists and nurses to antineoplastic agents. J Occup Med. 35(1):57-60.

McDiarmid MA, Egan T, Furio M, Bonacci M, Watts SR. 1986. Sampling for airborne fluorouracil in a hospital drug preparation area. Am J Hosp Pharm. 43(8):1942-1945.

Meijster T, Fransman W, Veldhof R, Kromhout H. 2006. Exposure to antineoplastic drugs outside the hospital environment. Ann Occup Hyg. 50(7):657-664.

http://dx.doi.org/10.1093/annhyg/mel023

Merger D, Tanguay C, Langlois E, Lefebvre M, Bussieres JF. 2013. Environmental contamination with methotrexate in Canadian community pharmacies. J Am Pharm Assoc. 53(4):423-426. http://dx.doi.org/10.1331/JAPhA.2013.12245

Merger D, Tanguay C, Langlois E, Lefebvre M, Bussieres JF. 2014. Multicenter study of environmental contamination with antineoplastic drugs in 33 Canadian hospitals. Int Arch Occup Environ Health. 87(3):307-313. http://dx.doi.org/10.1007/s00420-013-0862-0

Micoli G, Turci R, Arpellini M, Minoia C. 2001. Determination of 5-fluorouracil in environmental samples by solid-phase extraction and high-performance liquid chromatography with ultraviolet detection. J Chromatogr B Biomed Sci Appl. 750(1):25-32. http://dx.doi.org/10.1016/S0378-4347(00)00377-7

Minoia C, Turci R, Sottani C, Schiavi A, Perbellini L, Angeleri S, Draicchio F, Apostoli P. 1998. Application of high performance liquid chromatography/tandem mass spectrometry in the environmental and biological monitoring of health care personnel occupationally exposed to cyclophosphamide and ifosfamide. Rapid Commun Mass Spectrom. 12(20):1485-1493. http://dx.doi.org/10.1002/(SICI)1097-0231(19981030)12:20<1485::AID-RCM333>3.0.CO;2-N

Miyake T, Iwamoto T, Tanimura M, Okuda M. 2013. Impact of closed-system drug transfer device on exposure of environment and healthcare provider to cyclophosphamide in Japanese hospital. Springerplus. 2(1):273. http://dx.doi.org/10.1186/2193-1801-2-273 
Moretti M, Grollino MG, Pavanello S, Bonfiglioli R, Villarini M, Appolloni M, Carrieri M, Sabatini L, Dominici L, Stronati L et al. 2015. Micronuclei and chromosome aberrations in subjects occupationally exposed to antineoplastic drugs: A multicentric approach. Int Arch Occup Environ Health. 88(6):683-695. http://dx.doi.org/10.1007/s00420-014-0993-y

Naito T, Osawa T, Suzuki N, Goto T, Takada A, Nakamichi H, Onuki Y, Imai K, Nakanishi K, Kawakami J. 2012. Comparison of contamination levels on the exterior surfaces of vials containing platinum anticancer drugs in Japan. Biol Pharm Bull. 35(11):2043-2049. http://dx.doi.org/10.1248/bpb.b12-00628

Naslund Andreasson S, Anundi H, Thoren SB, Ehrsson H, Mahteme H. 2010. Is platinum present in blood and urine from treatment givers during hyperthermic intraperitoneal chemotherapy? J Oncol. 2010:649719. http://dx.doi.org/10.1155/2010/649719

Ndaw S, Denis F, Marsan P, d'Almeida A, Robert A. 2010. Biological monitoring of occupational exposure to 5-fluorouracil: Urinary alpha-fluoro-beta-alanine assay by high performance liquid chromatography tandem mass spectrometry in health care personnel. $\mathrm{J}$ Chromatogr B Analyt Technol Biomed Life Sci. 878(27):2630-2634. http://dx.doi.org/10.1016/j.jchromb.2010.02.011

Nussbaumer S, Geiser L, Sadeghipour F, Hochstrasser D, Bonnabry P, Veuthey JL, FleurySouverain S. 2012. Wipe sampling procedure coupled to LC-MS/MS analysis for the simultaneous determination of 10 cytotoxic drugs on different surfaces. Anal Bioanal Chem. 402(8):2499-2509. http://dx.doi.org/10.1007/s00216-011-5157-2

Nygren O, Gustavsson B, Strom L, Eriksson R, Jarneborn L, Friberg A. 2002. Exposure to anticancer drugs during preparation and administration. Investigations of an open and a closed system. J Environ Monit. 4(5):739-742. http://dx.doi.org/10.1039/B205132J

Nygren O, Lundgren C. 1997. Determination of platinum in workroom air and in blood and urine from nursing staff attending patients receiving cisplatin chemotherapy. Int Arch Occup Environ Health. 70(3):209-214. http://dx.doi.org/10.1007/s004200050209

Nyman HA, Jorgenson JA, Slawson MH. 2007. Workplace contamination with antineoplastic agents in a new cancer hospital using a closed-system drug transfer device. Hosp Pharm. 42(3):219-225. http://dx.doi.org/10.1310/hpj4203-219

Odraska P, Dolezalova L, Kuta J, Oravec M, Piler P, Blaha L. 2013. Evaluation of the efficacy of additional measures introduced for the protection of healthcare personnel handling antineoplastic drugs. Ann Occup Hyg. 57(2):240-250. http://dx.doi.org/10.1093/annhyg/mes057

Odraska P, Dolezalova L, Kuta J, Oravec M, Piler P, Synek S, Blaha L. 2014. Association of surface contamination by antineoplastic drugs with different working conditions in hospital pharmacies. Arch Environ Occup Health. 69(3):148-158.

http://dx.doi.org/10.1080/19338244.2013.763757

Odraska P, Dolezalova L, Piler P, Oravec M, Blaha L. 2011. Utilization of the solid sorbent media in monitoring of airborne cyclophosphamide concentrations and the implications for occupational hygiene. J Environ Monit. 13(5):1480-1487. http://dx.doi.org/10.1039/c0em00660b 
Page EH, Couch JR, De Perio MA. 2015. Evaluation of a multiple sclerosis cluster among nurses in an inpatient oncology ward. J Occup Environ Hyg. 12(5):D54-D59.

http://dx.doi.org/10.1080/15459624.2014.989359

Panahi D, Azari M, Akbari ME, Zendehdel R, Mirzaei HR, Hatami H, Mehrabi Y. 2016. Development of a new method for sampling and monitoring oncology staff exposed to cyclophosphamide drug. Environ Monit Assess. 188(4):238. http://dx.doi.org/10.1007/s10661$\underline{016-5255-X}$

Peelen S, Roeleveld N, Heederik D, Kromhout H, De Kort W. 1999. Reproductietoxische effecten bij ziekenhuispersoneel [Toxic effects on reproduction in hospital personnel]. The Hague, Netherlands: Dutch Ministry of Social Affairs and Employment.

Pethran A, Schierl R, Hauff K, Grimm CH, Boos KS, Nowak D. 2003. Uptake of antineoplastic agents in pharmacy and hospital personnel. Part I: Monitoring of urinary concentrations. Int Arch Occup Environ Health. 76(1):5-10. http://dx.doi.org/10.1007/s00420-002-0383-8

Pieri M, Castiglia L, Basilicata P, Sannolo N, Acampora A, Miraglia N. 2010. Biological monitoring of nurses exposed to doxorubicin and epirubicin by a validated liquid chromatography/fluorescence detection method. Ann Occup Hyg. 54(4):368-376. http://dx.doi.org/10.1093/annhyg/meq006

Pilger A, Kohler I, Stettner H, Mader RM, Rizovski B, Terkola R, Diem E, Franz-Hainzl E, Konnaris C, Valic E et al. 2000. Long-term monitoring of sister chromatid exchanges and micronucleus frequencies in pharmacy personnel occupationally exposed to cytostatic drugs. Int Arch Occup Environ Health. 73(7):442-448. http://dx.doi.org/10.1007/s004200000164

Poupeau C, Tanguay C, Caron NJ, Bussieres JF. 2016. Multicenter study of environmental contamination with cyclophosphamide, ifosfamide, and methotrexate in 48 Canadian hospitals. J Oncol Pharm Pract. http://dx.doi.org/10.1177/1078155216676632

Poupeau C, Tanguay C, Plante C, Gagne S, Caron N, Bussieres JF. 2017. Pilot study of biological monitoring of four antineoplastic drugs among Canadian healthcare workers. J Oncol Pharm Pract. 23(5):323-332. http://dx.doi.org/10.1177/1078155216643860

Power L, Sessink P, Gesy K, Charbonneau F. 2014. Hazardous drug residue on exterior vial surfaces: Evaluation of a commercial manufacturing process. Hosp Pharm. 49(4):355-362.

Pyy L, Sorsa M, Hakala E. 1988. Ambient monitoring of cyclophosphamide in manufacture and hospitals. Am Ind Hyg Assoc J. 49(6):314-317. http://dx.doi.org/10.1080/15298668891379800

Ramphal R, Bains T, Goulet G, Vaillancourt R. 2015. Occupational exposure to chemotherapy of pharmacy personnel at a single centre. Can J Hosp Pharm. 68(2):104-112.

Ramphal R, Bains T, Vaillancourt R, Osmond MH, Barrowman N. 2014. Occupational exposure to cyclophosphamide in nurses at a single center. J Occup Environ Med. 56(3):304-312. http://dx.doi.org/10.1097/JOM.0000000000000097

Rekhadevi PV, Sailaja N, Chandrasekhar M, Mahboob M, Rahman MF, Grover P. 2007. Genotoxicity assessment in oncology nurses handling anti-neoplastic drugs. Mutagenesis. 22(6):395-401. http://dx.doi.org/10.1093/mutage/gem032 
Rubino FM, Verduci C, Buratti M, Fustinoni S, Campo L, Omodeo-Sale E, Giglio M, Iavicoli S, Brambilla G, Colombi A. 2006. Assay of urinary alpha-fluoro-beta-alanine by gas chromatography-mass spectrometry for the biological monitoring of occupational exposure to 5fluorouracil in oncology nurses and pharmacy technicians. Biomed Chromatogr. 20(3):257-266. http://dx.doi.org/10.1002/bmc.559

Sabatini L, Barbieri A, Lodi V, Violante FS. 2012. Biological monitoring of occupational exposure to antineoplastic drugs in hospital settings. Med Lav. 103(5):394-401.

Schierl R, Masini C, Groeneveld S, Fischer E, Bohlandt A, Rosini V, D P. 2016. Environmental contamination by cyclophosphamide preparation: Comparison of conventional manual production in biological safety cabinet and robot-assisted production by APOTECAchemo. $\mathrm{J}$ Oncol Pharm Pract. 22(1):37-45. http://dx.doi.org/10.1177/1078155214551316

Schierl R, Bohlandt A, Nowak D. 2009. Guidance values for surface monitoring of antineoplastic drugs in German pharmacies. Ann Occup Hyg. 53(7):703-711. http://dx.doi.org/10.1093/annhyg/mep050

Schierl R, Herwig A, Pfaller A, Groebmair S, Fischer E. 2010. Surface contamination of antineoplastic drug vials: Comparison of unprotected and protected vials. Am J Health Syst Pharm. 67(6):428-429. http://dx.doi.org/10.2146/ajhp080621

Schierl R, Novotna J, Piso P, Bohlandt A, Nowak D. 2012. Low surface contamination by cis/oxaliplatin during hyperthermic intraperitoneal chemotherapy (HIPEC). Eur J Surg Oncol. 38(1):88-94. http://dx.doi.org/10.1016/j.ejso.2011.10.009

Schmaus G, Schierl R, Funck S. 2002. Monitoring surface contamination by antineoplastic drugs using gas chromatography-mass spectrometry and voltammetry. Am J Health Syst Pharm. 59(10):956-961.

Schmid K, Boettcher MI, Pelz JO, Meyer T, Korinth G, Angerer J, Drexler H. 2006. Investigations on safety of hyperthermic intraoperative intraperitoneal chemotherapy (HIPEC) with Mitomycin C. Eur J Surg Oncol. 32(10):1222-1225.

http://dx.doi.org/10.1016/j.ejso.2006.05.011

Schreiber C, Radon K, Pethran A, Schierl R, Hauff K, Grimm CH, Boos KS, Nowak D. 2003. Uptake of antineoplastic agents in pharmacy personnel. Part II: Study of work-related risk factors. Int Arch Occup Environ Health. 76(1):11-16. http://dx.doi.org/10.1007/s00420-002$\underline{0385-6}$

Schulz H, Bigelow S, Dobish R, Chambers CR. 2005. Antineoplastic agent workplace contamination study: The Alberta Cancer Board Pharmacy perspective. J Oncol Pharm Pract. 11(3):101-109. http://dx.doi.org/10.1191/1078155205jp152oa

Sessink PJ, Anzion RB, Van den Broek PH, Bos RP. 1992. Detection of contamination with antineoplastic agents in a hospital pharmacy department. Pharm Weekbl Sci. 14(1):16-22.

Sessink PJ, Boer KA, Scheefhals AP, Anzion RB, Bos RP. 1992. Occupational exposure to antineoplastic agents at several departments in a hospital. Environmental contamination and 
excretion of cyclophosphamide and ifosfamide in urine of exposed workers. Int Arch Occup Environ Health. 64(2):105-112. http://dx.doi.org/10.1007/BF00381477

Sessink PJ, Cerna M, Rossner P, Pastorkova A, Bavarova H, Frankova K, Anzion RB, Bos RP. 1994a. Urinary cyclophosphamide excretion and chromosomal aberrations in peripheral blood lymphocytes after occupational exposure to antineoplastic agents. Mutat Res. 309(2):193-199. http://dx.doi.org/10.1016/0027-5107(94)90092-2

Sessink PJ, Connor TH, Jorgenson JA, Tyler TG. 2011. Reduction in surface contamination with antineoplastic drugs in 22 hospital pharmacies in the US following implementation of a closedsystem drug transfer device. J Oncol Pharm Pract. 17(1):39-48.

http://dx.doi.org/10.1177/1078155210361431

Sessink PJ, Friemel NS, Anzion RB, Bos RP. 1994b. Biological and environmental monitoring of occupational exposure of pharmaceutical plant workers to methotrexate. Int Arch Occup Environ Health. 65(6):401-403. http://dx.doi.org/10.1007/BF00383251

Sessink PJ, Leclercq GM, Wouters DM, Halbardier L, Hammad C, Kassoul N. 2015. Environmental contamination, product contamination and workers exposure using a robotic system for antineoplastic drug preparation. J Oncol Pharm Pract. 21(2):118-127. http://dx.doi.org/10.1177/1078155214522840

Sessink PJ, Timmersmans JL, Anzion RB, Bos RP. 1994. Assessment of occupational exposure of pharmaceutical plant workers to 5-fluorouracil. Determination of alpha-fluoro-beta-alanine in urine. J Occup Med. 36(1):79-83.

Sessink PJ, Trahan J, Coyne JW. 2013. Reduction in surface contamination with cyclophosphamide in 30 US hospital pharmacies following implementation of a closed-system drug transfer device. Hosp Pharm. 48(3):204-212. http://dx.doi.org/10.1310/hpj4803-204

Sessink PJ, Van de Kerkhof MC, Anzion RB, Noordhoek J, Bos RP. 1994c. Environmental contamination and assessment of exposure to antineoplastic agents by determination of cyclophosphamide in urine of exposed pharmacy technicians: Is skin absorption an important exposure route? Arch Environ Health. 49(3):165-169.

http://dx.doi.org/10.1080/00039896.1994.9940377

Sessink PJ, Wittenhorst BC, Anzion RB, Bos RP. 1997b. Exposure of pharmacy technicians to antineoplastic agents: Reevaluation after additional protective measures. Arch Environ Health. 52(3):240-244. http://dx.doi.org/10.1080/00039899709602893

Sessink PJM, Joost HCdR, Pierik FH, Anzion RBM, Bos RP. 1993. Occupational exposure of animal caretakers to cyclophosphamide. J Occup Med. 35(1):47-52.

Siderov J, Kirsa S, McLauchlan R. 2009. Surface contamination of cytotoxic chemotherapy preparation areas in Australian Hospital Pharmacy Departments. J Pharm Pract Res. 39(2):117121. http://dx.doi.org/10.1002/j.2055-2335.2009.tb00434.x

Siderov J, Kirsa S, McLauchlan R. 2010. Reducing workplace cytotoxic surface contamination using a closed-system drug transfer device. J Oncol Pharm Pract. 16(1):19-25.

http://dx.doi.org/10.1177/1078155209352543 
Solass W, Giger-Pabst U, Zieren J, Reymond MA. 2013. Pressurized intraperitoneal aerosol chemotherapy (PIPAC): Occupational health and safety aspects. Ann Surg Oncol. 20(11):35043511. http://dx.doi.org/10.1245/s10434-013-3039-x

Sorsa M, Pyy L, Salomaa S, Nylund L, Yager JW. 1988. Biological and environmental monitoring of occupational exposure to cyclophosphamide in industry and hospitals. Mutat Res. 204(3):465-479. http://dx.doi.org/10.1016/0165-1218(88)90042-0

Sottani C, Porro B, Comelli M, Imbriani M, Minoia C. 2010. An analysis to study trends in occupational exposure to antineoplastic drugs among health care workers. J Chromatogr B Analyt Technol Biomed Life Sci. 878(27):2593-2605.

http://dx.doi.org/10.1016/j.jchromb.2010.04.030

Sottani C, Porro B, Imbriani M, Minoia C. 2012. Occupational exposure to antineoplastic drugs in four Italian health care settings. Toxicol Lett. 213(1):107-115.

http://dx.doi.org/10.1016/j.toxlet.2011.03.028

Sottani C, Rinaldi P, Leoni E, Poggi G, Teragni C, Delmonte A, Minoia C. 2008. Simultaneous determination of cyclophosphamide, ifosfamide, doxorubicin, epirubicin and daunorubicin in human urine using high-performance liquid chromatography/electrospray ionization tandem mass spectrometry: Bioanalytical method validation. Rapid Commun Mass Spectrom. 22(17):2645-2659. http://dx.doi.org/10.1002/rcm.3657

Stover D, Achutan C. 2011. Occupational exposures to antineoplastic drugs in an OncologyHematology Department. J Occup Environ Hyg. 8(1):D1-6.

http://dx.doi.org/10.1080/15459624.2011.537510

Stuart OA, Stephens AD, Welch L, Sugarbaker PH. 2002. Safety monitoring of the coliseum technique for heated intraoperative intraperitoneal chemotherapy with mitomycin C. Ann Surg Oncol. 9(2):186-191. http://dx.doi.org/10.1007/BF02557372

Sugiura S, Asano M, Kinoshita K, Tanimura M, Nabeshima T. 2011. Risks to health professionals from hazardous drugs in Japan: A pilot study of environmental and biological monitoring of occupational exposure to cyclophosphamide. J Oncol Pharm Pract. 17(1):14-19. https://doi.org/10.1177/1078155209358632

Sugiura S, Nakanishi H, Asano M, Hashida T, Tanimura M, Hama T, Nabeshima T. 2011. Multicenter study for environmental and biological monitoring of occupational exposure to cyclophosphamide in Japan. J Oncol Pharm Pract. 17(1):20-28. http://dx.doi.org/10.1177/1078155210369851

Tanimura M, Yamada K, Sugiura S, Mori K, Nagata H, Tadokoro K, Miyake T, Hamaguchi Y, Sessink P, Nabeshima T. 2009. An environmental and biological study of occupational exposure to cyclophosphamide in the pharmacy of a Japanese community hospital designated for the treatment of cancer. J Health Sci. 55(5):750-756. http://dx.doi.org/10.1248/jhs.55.750

Thulin H, Sundberg E, Hansson K, Cole J, Hartley-Asp B. 1995. Occupational exposure to nornitrogen mustard: Chemical and biological monitoring. Toxicol Ind Health. 11(1):89-97. http://dx.doi.org/10.1177/074823379501100108 
Touzin K, Bussieres JF, Langlois É, Lefebvre M. 2009. Evaluation of surface contamination in a hospital hematology-oncology pharmacy. J Onc Pharm Pract. 15(1):53-61.

http://dx.doi.org/10.1177/1078155208096904

Touzin K, Bussieres JF, Langlois E, Lefebvre M, Gallant C. 2008. Cyclophosphamide contamination observed on the external surfaces of drug vials and the efficacy of cleaning on vial contamination. Ann Occup Hyg. 52(8):765-771. http://dx.doi.org/10.1093/annhyg/men050

Turci R, Minoia C, Sottani C, Coghi R, Severi P, Castriotta C, Del Bianco M, Imbriani M. 2011. Occupational exposure to antineoplastic drugs in seven Italian hospitals: The effect of quality assurance and adherence to guidelines. J Oncol Pharm Pract. 17(4):320-332.

http://dx.doi.org/10.1177/1078155210381931

Turci R, Sottani C, Ronchi A, Minoia C. 2002. Biological monitoring of hospital personnel occupationally exposed to antineoplastic agents. Toxicol Lett. 134(1-3):57-64.

http://dx.doi.org/10.1016/S0378-4274(02)00163-7

Ursini CL, Cavallo D, Colombi A, Giglio M, Marinaccio A, Iavicoli S. 2006. Evaluation of early DNA damage in healthcare workers handling antineoplastic drugs. Int Arch Occup Environ Health. 80(2):134-140. http://dx.doi.org/10.1007/s00420-006-0111-X

Van Vloten WA, Cooijmans AC, Poel J, Meulenbelt J. 1993. Concentrations of nitrogen mustard in the air during topical treatment of patients with mycosis fungoides. Br J Dermatol. 128(4):404-406. http://dx.doi.org/10.1111/j.1365-2133.1993.tb00199.x

Vandenbroucke J, Robays H. 2001. How to protect environment and employees against cytotoxic agents, the UZ Ghent experience. J Oncol Pharm Practice. 6:146-152. http://dx.doi.org/10.1177/107815520100600403

Venitt S, Crofton-Sleigh C, Hunt J, Speechley V, Briggs K. 1984. Monitoring exposure of nursing and pharmacy personnel to cytotoxic drugs: Urinary mutation assays and urinary platinum as markers of absorption. Lancet. 323(8368):74-77. http://dx.doi.org/10.1016/S0140$\underline{6736(84) 90005-9}$

Viegas S, Padua M, Veiga AC, Carolino E, Gomes M. 2014. Antineoplastic drugs contamination of workplace surfaces in two Portuguese hospitals. Environ Monit Assess. 186(11):7807-7818. http://dx.doi.org/10.1007/s10661-014-3969-1

Villa AF, El Balkhi S, Aboura R, Sageot H, Hasni-Pichard H, Pocard M, Elias D, Joly N, Payen D, Blot F et al. 2015. Evaluation of oxaliplatin exposure of healthcare workers during heated intraperitoneal perioperative chemotherapy (HIPEC). Ind Health. 53(1):28-37. http://dx.doi.org/10.2486/indhealth.2014-0025

Villarini M, Dominici L, Piccinini R, Fatigoni C, Ambrogi M, Curti G, Morucci P, Muzi G, Monarca S, Moretti M. 2011. Assessment of primary, oxidative and excision repaired DNA damage in hospital personnel handling antineoplastic drugs. Mutagenesis. 26(3):359-369. http://dx.doi.org/10.1093/mutage/geq102 
Vyas N, Turner A, Clark JM, Sewell GJ. 2016. Evaluation of a closed-system cytotoxic transfer device in a pharmaceutical isolator. J Oncol Pharm Pract. 22(1):10-19.

http://dx.doi.org/10.1177/1078155214544993

Wick C, Slawson MH, Jorgenson JA, Tyler LS. 2003. Using a closed-system protective device to reduce personnel exposure to antineoplastic agents. Am J Health Syst Pharm. 60(22):2314-2320.

Yoshida J, Koda S, Nishida S, Nakano H, Tei G, Kumagai S. 2013. Association between occupational exposure and control measures for antineoplastic drugs in a pharmacy of a hospital. Ann Occup Hyg. 57(2):251-260. https://doi.org/10.1093/annhyg/mes061

Yoshida J, Tei G, Mochizuki C, Masu Y, Koda S, Kumagai S. 2009. Use of a closed system device to reduce occupational contamination and exposure to antineoplastic drugs in the hospital work environment. Ann Occup Hyg. 53(2):153-160. http://dx.doi.org/10.1093/annhyg/men081

Yuki M, Ishida T, Sekine S. 2015. Secondary exposure of family members to cyclophosphamide after chemotherapy of outpatients with cancer: A pilot study. Oncol Nurs Forum. 42(6):665-671. http://dx.doi.org/10.1188/15.ONF.42-06AP

Yuki M, Sekine S, Takase K, Ishida T, Sessink PJ. 2012. Exposure of family members to antineoplastic drugs via excreta of treated cancer patients. J Oncol Pharm Pract. http://dx.doi.org/10.1177/1078155212459667

Ziegler E, Mason HJ, Baxter PJ. 2002. Occupational exposure to cytotoxic drugs in two UK oncology wards. Occup Environ Med. 59(9):608-612. http://dx.doi.org/10.1136/oem.59.9.608 


\section{Appendix H. Protocol History}

The original and revised protocols are available in Appendix I.

Table H-1. Protocol History and Revisions

\begin{tabular}{cl}
\hline Date & Activity or Revision \\
\hline
\end{tabular}

May 28, 2015

October 9, 2015

December 7, 2018
Draft evaluation protocol reviewed: sent to experts for comment/review

Evaluation protocol posted on Office of Health Assessment and Translation website

Revised protocol posted to reflect principal updates made during evaluation with justifications noted (date implemented):

1. Decision to evaluate evidence of all health effects, including reproductive and developmental toxicity, to make the evaluation comprehensive and ensure that the most recent papers on reproductive system effects were included (January 2016).

2. Consideration of acute effects as well as genetic toxicity as primary health outcomes, not secondary health outcomes. Genetic toxicity moved to primary health outcome because of the established association of chromosome aberrations and micronucleus induction with future cancer incidence. These changes were made based on consultation with a technical advisor and federal experts following review of a preliminary draft on the monograph (November 2016).

3. Adjustment of exposure characterization risk-of-bias assessment to include job position with work tasks and details about drug handling (e.g., types of cancer chemotherapy agents, frequency of drugs handled) via questionnaire or self-reported daily diary with validation as probably low risk of bias. These changes were made based on consultation with a technical advisor and federal experts following review of a preliminary draft on the monograph (November 2016).

4. Adjustment of initial confidence rating in the body of evidence of cross-sectional studies of genetic toxicity from low initial confidence to moderate initial confidence because the three genetic toxicity outcomes are rapidly developing (e.g., hours to days) and the period of occupational exposure began prior to development of the outcome (January 2017).

5. Removal of the hazard identification step due to lack of a formal way to integrate authoritative reviews into the integration of evidence streams step (January 2017). Note, given that level of evidence was moderate and no hazard conclusions reached, a formal public comment period was not included 


\section{Appendix I. Supplemental Files}

The following supplemental files are available at https://doi.org/10.22427/NTP-DATAMONOGRAPH5.

\section{I.1. Protocol Information}

Protocol

Protocol.pdf

Revised Protocol

Revised_Protocol.pdf

\section{I.2. Monitoring References}

Supplemental Table 1. Environmental Monitoring References

Supplemental Table_1 Environmental Monitoring references_final.xlsx

Supplemental Table 2. Biomonitoring References

Supplemental Table_2 Biomonitoring references_final.xlsx 
National Toxicology Program

NTP Central Data Management, MD K2-05

National Institute of Environmental Health Sciences

P.O. Box 12233

Research Triangle Park, NC 27709

http://ntp.niehs.nih.gov 